Phonological

Augmentation in

Prominent Positions

Jennifer L. Smith

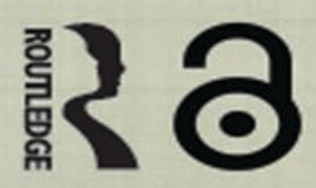




\section{PHONOLOGICAL AugMENTATION IN PROMINENT POSITIONS}

\section{Jennifer L. Smith}

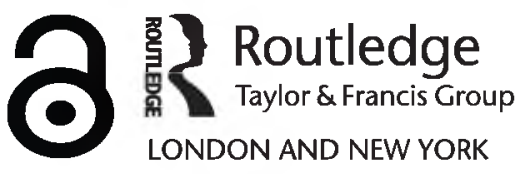


First published 2005 by Routledge

Published 2017 by Routledge

2 Park Square, Milton Park, Abingdon, Oxon OX14 4RN

711 Third Avenue, New York, NY 10017, USA

Routledge is an imprint of the Taylor \& Francis Group, an informa business

Copyright $\odot 2005$ Taylor \& Francis

The Open Access version of this book, available at www.tandfebooks.com, has been made available under a Creative Commons Attribution-Non Commercial-No Derivatives 4.0 license.

Catalog record is available from the Library of Congress

ISBN 978-0-415-97107-2 (hbk)

ISBN 978-0-415-86149-6 (pbk) 
for my family 


\section{Contents}

PREFACE

$i x$

ACKNOWLEDGMENTS

$x i$

1. POSITIONAL AUGMENTATION: MARKEDNESS CONSTRAINTS FOR PROMINENT POSITIONS

1.1 Introduction

1.2 Synopsis of the proposal

1.3 Strong and weak positions in phonological analysis 13

1.4 Outline of the dissertation 23

2. A THEORY OF POSITIONAL AUGMENTATION CONSTRAINTS 25

2.1 Introduction 25

2.2 Formal models of constraints and CON 26

$2.3 \mathrm{M} / \mathrm{str}$ constraints and the Prominence Condition 42

$2.4 \mathrm{M} / \Psi$ str constraints and the Segmental Contrast Condition $\quad 81$

2.5 Summary and conclusions 93

3. AUGMENTATION OF PHONETICALLY STRONG POSITIONS 95

3.1 Introduction 95

3.2 Positional augmentation in stressed syllables 96

3.3 Positional augmentation in long vowels 137

3.4 Positional augmentation in syllable onsets 140

3.5 Conclusion: Predicted and attested M/Фstr constraints 143 
4. AUGMENTATION OF PSYCHOLINGUISTICALLY STRONG POSITIONS

4.1 Introduction 147

4.2 Exemplification of M/Tstr constraints 149

4.3 Psycholinguistic evidence behind the Segmental Contrast Condition

4.4 Defining "initial syllable"

4.5 Summary and conclusions

5. POSITIONAL AUGMENTATION AND POSITIONAL NEUTRALIZATION

5.1 Introduction

5.2 Positional neutralization in Optimality Theory

5.3 Implications of distinguishing positional augmentation and neutralization

5.4 Summary and conclusions

6. CONCLUSIONS, IMPLICATIONS, AND FUTURE DIRECTIONS 273

BIBLIOGRAPHY

INDEX OF NAMES

INDEX OF LANGUAGES 


\section{Preface}

This book develops an account of markedness constraints that apply exclusively to material in phonologically prominent or "strong" positions, called here $\mathbf{M} / \mathbf{s t r}(\mathbf{o n g})$ constraints. The model that is proposed in order to distinguish between possible and impossible $\mathbf{M} / \mathbf{s t r}$ constraints has implications for the nature of the interaction between formal factors and substantive or functional factors in phonology.

$\mathbf{M} / \mathbf{s t r}$ constraints are subject to two substantively based restrictions. The first is the Prominence Condition, which states that an $\mathbf{M} / \mathbf{s t r}$ constraint is legitimate only if its satisfaction enhances perceptual salience. For example, an M/str constraint demanding high-sonority nuclei in the strong position stressed syllable is legitimate, but a constraint that simply bans a typologically marked feature value in some strong position is not. The Prominence Condition correctly predicts that all $\mathbf{M} / \mathbf{s t r}$ constraints are prominence-enhancing or AUGMENTATION constraints.

The second restriction, the Segmental Contrast Condition, applies to $\mathbf{M} / \mathbf{s t r}$ constraints on positions that are strong for psycholinguistic (as opposed to phonetic) reasons. This restriction has its basis in the importance of psycholinguistically strong positions for early-stage word recognition. It prohibits any $\mathbf{M} / \mathbf{s t r}$ constraint from referring to a psycholinguistically strong position if its satisfaction would impede early-stage word recognition, such as by neutralizing segmental feature contrasts (except for those that improve left-edge demarcation, which potentially facilitates word recognition). Thus, although an $\mathbf{M} / \mathbf{s t r}$ constraint calling for high-sonority nuclei in the psycholinguistically strong position initial syllable would pass the Prominence Condition, it is banned by the Segmental Contrast Condition, because its satisfaction neutralizes a segmental contrast that is not at the left edge.

The Prominence Condition and the Segmental Contrast Condition are formally implemented as filters on the output of generalized constraint-building schemas, determining which of the logically possible $\mathbf{M} / \mathbf{s t r}$ constraints are actually included in the universal constraint set CON. In an extension of Inductive Grounding (Hayes 
1999a), these and other constraint filters are viewed as the locus of functional grounding in the formal phonological system. This Schema/Filter model of CON allows the constraint set to reflect substantive phonetic and psycholinguistic factors, while maintaining a view of phonology as a formal system that manipulates formal objects-including constraints and the basic phonological elements from which they are constructed-without necessarily having access to every fine-grained detail of articulation, acoustics, perception, and processing. 


\section{Acknowledgments}

This is a slightly revised version of my University of Massachusetts, Amherst, dissertation, which was submitted in May 2002. As such, first and foremost, I would like to give deep and heartfelt thanks to the members of my dissertation committee: Mark Feinstein, John Kingston, Joe Pater, Lisa Selkirk, and especially John McCarthy, who served as chair. Many thanks also to the other UMass Linguistics faculty members, particularly Lyn Frazier, Roger Higgins, Kyle Johnson, and Angelika Kratzer, whose approaches to linguistics have also influenced this project, and to Kathy Adamczyk and Lynne Ballard for keeping me organized. Research for the dissertation was partially supported by the National Science Foundation under grant SBR-9420424 and by an NSF Graduate Research Fellowship.

I am grateful for many phonology and phonetics conversations at UMass with, especially, Jill Beckman, Laura Benua, John Alderete, Elliott Moreton, Katy Carlson, Caroline Jones, Cecilia Kirk, Ania Łubowicz, Steve Parker, Mariko Sugahara, Paul de Lacy, Nancy Hall, Meredith Landman, Maria Gouskova, Adam Werle, Andries Coetzee, and Ben Gelbart. In particular, Jill Beckman's work first inspired me to think about positional constraints, and work by Steve Parker and by Paul de Lacy has contributed to my understanding of markedness constraints on strong positions. Other phonologists and phoneticians whose input or influence has directly or indirectly contributed to this project include Abby Cohn, Katherine Crosswhite, Chip Gerfen, Heli Harrikari, Teruhiro Hayata, Dylan Herrick, Junko Itô, Ed Keer, Tomoyuki Kubo, Kazutaka Kurisu, Lisa Lavoie, Linda Lombardi, Armin Mester, Jaye Padgett, Alan Prince, Paul Smolensky, Caro Struijke, Adam Ussishkin, Rachel Walker, Andy Wedel, and Cheryl Zoll. Thanks also to audiences at the MIT Phonology Circle, BLS 26, HUMDRUM I, TREND 2000, UC Santa Cruz, UNC Chapel Hill, and the Stanford Phonology Interest Group, and to the participants in Phonology C, Spring 2001, UCSC. Very special thanks to Jaye 
Padgett at UCSC and Chip Gerfen at UNC Chapel Hill for feedback and large doses of moral support in the final stages of revision.

For life beyond linguistics, I owe thanks for support and good times to many people around UMass, especially-but certainly not exclusively-my classmates Katy Carlson, Maria Nella Carminati, Caroline Jones, Cecilia Kirk, Oliver Steele, and Mike Terry; my (non-classmate) housemates Ji-yung Kim and Mariko Sugahara; and Isadora Cohen, André Isaak, Eva Juarros, Kiyomi Kusumoto, Elliott Moreton, Janina Radó, and the Haymarket Café. Eternal gratitude goes to everyone in the Northampton Class of the Boston Branch of the Royal Scottish Country Dance Society, especially founding teacher Virginia Van Scoy and amazing fiddler and class clown Earl Gaddis, for preserving my sense of proportion.

Thanks to all at UC Santa Cruz and UNC Chapel Hill for making me feel so welcome; I have had the good fortune to work with many wonderful colleagues and students at both places.

Finally, thanks to my family, Paul, Ginnie, and Greg Smith, for letting me make my own choices and move at my own pace, and for all their love and support. 


\section{PHONOLOGICAL Augmentation IN \\ PROMINENT Positions}




\section{Positional Augmentation:}

\section{Markedness Constraints for Prominent Positions}

\subsection{Introduction}

Phonological requirements sometimes hold specifically of material in phonologically prominent or "strong" positions, such as stressed syllables or roots. Here, such requirements are analyzed in the framework of Optimality Theory (Prince \& Smolensky 1993; McCarthy \& Prince 1993ab, 1995) in terms of markedness constraints ${ }^{1}$ that make specific reference to strong positions (abbreviated $\mathbf{M} / \mathbf{s t r}(\mathbf{O}$ g)). It is shown that only certain kinds of requirements ever hold of strong positions, so the inclusion of all logically possible $\mathbf{M} / \mathbf{s t r}$ constraints in the universal constraint set (CON) would lead to incorrect typological predictions. The theory developed here is able to distinguish between those logically possible $\mathbf{M} / \mathbf{s t r}$ constraints that are actual constraints and those that are not.

The difference between legitimate and impossible $\mathbf{M} / \mathbf{s t r}$ constraints stems from two restrictions on constraints of this type-restrictions that are framed in functional or substantive terms. First, as shown in $\$ 2.3$, all $\mathbf{M} / \mathbf{s t r}$ constraints in CON are of a type called here augmentation constraints: constraints that call for the presence of perceptually prominent characteristics. Augmentation constraints can

1. A markedness constraint is any constraint that makes reference only to output forms; that is, any constraint for which information about input forms, or the correspondence relation between input and output forms, is irrelevant in determining the degree of violation. Such constraints are sometimes referred to in the OT literature as "phono-constraints," "structural constraints," or "well-formedness constraints." 
be relativized to strong positions because, although they are markedness (contrastneutralizing) constraints, their satisfaction further enhances these already prominent positions by increasing their perceptual salience. Second, M/str constraints affecting positions that are strong for psycholinguistic reasons (as opposed to phonetic reasons) are prohibited if their satisfaction would hinder early-stage word recognition (defined in $\S 4.3 .1$ ), such as by neutralizing segmental feature contrasts. This is because the privileged status of psycholinguistically strong positions is based on their important role in this aspect of speech processing. One of the results of this restriction is that certain augmentation constraints that can be relativized to the phonetically strong position stressed syllable cannot be relativized to the similarly sized initial syllable, since it is a psycholinguistically strong position.

Because the restrictions on $\mathbf{M} / \mathbf{s t r}$ constraints are substantive in nature, a theory of possible and impossible $\mathbf{M} / \mathbf{s t r}$ constraints must also address the question of how the phonology, a formal system that manipulates abstract formal objects, can nevertheless be shaped by substantive considerations. The conception of CON proposed here, called the Schema/Filter model, holds that constraints themselves are freely constructed from formal phonological primitives without reference to substantive considerations; functional grounding enters the phonology through the fact that only a subset of the formally possible constraints are actually included in CON (extending an idea advanced by Hayes (1999a) to model the influence of phonetic grounding on feature-context markedness constraints, such as *[nas][voi]). In the Schema/Filter model, constraints are freely constructed by the application of a number of general constraint schemas to the entire inventory of primitive phonological elements. However, constraint filters, which make use of perceptual, articulatory, or other extra-phonological information to screen constraints, permit only some of the formally constructed constraints into CoN. The substantive restrictions on $\mathbf{M} / \mathbf{s t r}$ constraints described above are imposed by two such constraint filters: the Prominence Condition, which rejects any $\mathbf{M} / \mathbf{s t r}$ constraint unless it calls for the enhancement of perceptual prominence, and the Segmental Contrast Condition, which rejects $\mathbf{M} / \mathbf{s t r}$ constraints for psycholinguistically strong positions if they inappropriately neutralize contrasts that are important in early-stage word recognition.

While the proposal developed here addresses the specific problem of the difference between possible and impossible $\mathbf{M} / \mathbf{s t r}$ constraints, the languages examined below also provide several pieces of evidence that phonology is an abstract and formal system despite the influence of substantive considerations on many phonological constraints and elements. For example, the existence of both the constraint ONSET, which requires syllables to have onsets, and the * ONSET/X subhierarchy of constraints, which collectively require syllable onsets to be low in sonority, can be related to the substantive fact that interspersing low-sonority 
elements between vowels enhances the perceptibility of the vowels. However, ONSET and *ONSET/X are formally distinct in that they evaluate different aspects of syllable structure and in that they can be freely ranked with respect to one another (\$2.3.2.3). Another piece of evidence for the formal nature of phonology can be seen in the variety of $\mathbf{M} / \mathbf{s t r}$ constraints that apply to phonetically strong positions; while it is a particular phonetic characteristic that gives a phonetically strong position its special status, the position can nevertheless be the target of an $\mathbf{M} / \mathbf{s t r}$ constraint that manipulates properties unrelated to that phonetic characteristic. This fact indicates that although the status of "phonetically strong position" has a substantive basis, it functions as an abstract phonological property (§2.4.3). The Schema/Filter model of CON predicts such results-although this model explicitly includes substantive restrictions on the phonological system, it nevertheless maintains a formal view of phonology.

The following section $(\S 1.2)$ presents a more detailed overview of the proposal sketched above, indicating where in subsequent chapters each aspect of the proposal is addressed. Then, $\S 1.3$ makes explicit the theoretical background presupposed in this dissertation, presenting evidence for the existence of phonologically strong positions ( $\$ 1.3 .1)$ and arguing that $\mathbf{M} / \mathbf{s t r}$ constraints, rather than $\mathbf{F} / \mathbf{w k}$ constraints, are the best way to account for phonological requirements on strong positions (§1.3.2).

\subsection{Synopsis of the proposal}

This section first establishes the empirical context of the proposal developed in this dissertation by presenting the problem posed by $\mathbf{M} / \mathbf{s t r}$ constraints - they exist, but they must be restricted, or incorrect typological predictions are made (§1.2.1). An overview of the proposal is then given: the Prominence Condition ( $\$ 1.2 .2 .1)$, the Segmental Contrast Condition (\$1.2.2.2), and their place in the Schema/Filter model of CON (§1.2.2.3).

\subsubsection{The problem: $M /$ str constraints and typological predictions}

When a phonological requirement holds specifically of a strong position, we know that there is a markedness constraint, enforcing that requirement, that is relativized 
to the strong position in question. ${ }^{2}$ Examples of such requirements, and the $\mathbf{M} / \mathbf{s t r}$ constraints that enforce them, are given in (1); these examples are discussed in more detail in chapters 3 and 4.

(1) Markedness constraints for strong positions

(a) Stressed syllables must be heavy

ه Mohawk: Open stressed syllables are lengthened (Michelson 1988)

k-atirút-ha? 1A-pull-HAB 'I pull'

$\Lambda$-k-atirú.t. $-\wedge$ ? FUT-1A-pull-PUNC 'I'll pull'

M/str constraint: HEAVYo/ó (\$3.2.1)

(b) Stressed syllables must have low-sonority onsets

D Pirahã: Stress is attracted to syllables with voiceless-obstruent onsets (Everett \& Everett 1984ab, Everett 1988)

bii.sái 'red' (Note: acute accent marks stress;

?á.ba.gi 'toucan' underline indicates high tone)

M/str constraint: [*ONSET/X]/ó (§3.2.2)

(c) Long vowels must have high-sonority nuclei

D Yawelmani: Long high vowels are lowered (Kuroda 1967; Kisseberth 1969)

$$
\begin{array}{ll}
\text { c'o:m-al } & \text { 'might destroy' /c'u:m + al/ } \\
\text { so:g-al } & \text { 'might pull out the cork' } / \text { su:g + al/ }
\end{array}
$$

M/str constraint: [*PEAK/X]/V: (§3.3)

2. See $\$ 1.3 .2$ for why phonological requirements specific to strong positions are analyzed in terms of $\mathbf{M} / \mathbf{s t r}$ constraints. 
(d) Initial syllables must have onsets

D Arapaho: Words are always consonant-initial (Salzmann 1956)

$\begin{array}{ll}\text { xooó } & \text { 'skunk' } \\ \text { hé } \theta & \text { 'dog' } \\ \text { nówor } & \text { 'fish' } \\ \text { *owor } & \end{array}$

M/str constraint: ONSET/ $\sigma_{1}(\S 4.2 .1)$

(e) Roots must bear stress

D Tuyuca: Default stress is inserted on root-final vowel (Barnes 1996) /hoo + a/ hoóa (Note: root segments are underlined) submerge.oneself-EV

'I submerge myself'

M/str constraint: HAVESTRESS/Root (\$4.2.2)

The M/str constraints in (1) are attested, and necessary, members of the universal constraint set CON (see chapters 3 and 4 for phonological analyses of these and other languages in which such constraints are active). However, not just any markedness constraint can be relativized to strong positions. For example, if an ordinary featural markedness constraint such as *MIDV ('output forms do not contain mid vowels') were given an $\mathbf{M} / \mathbf{s t r}$ counterpart specific to stressed syllables, *MIDV/ó, then the ranking shown in (2) would be a possible ranking, predicted to occur in some language.

(2) Hypothetical feature-markedness $\mathbf{M} / \mathbf{s t r}$ constraint: *MIDV/ó

\begin{tabular}{|r||c|c|c|c|}
\hline Input: /tépo/ & $*$ MidV/ơ & $\begin{array}{c}\text { IDENT } \\
\text { [Vht]/ó }\end{array}$ & IDENT[Vht] & $*$ MidV \\
\hline \hline a. tépo & $* !$ & & & $* *$ \\
\hline b. típo & & $*$ & $*$ & $*$ \\
\hline c. típu & & $*$ & $* * !$ & \\
\hline
\end{tabular}


In OT, the possibilities for phonological contrast in a particular language are determined by the relative ranking in that language of markedness constraints, which require outputs to be free of complex or otherwise dispreferred phonological structures, and faithfulness constraints, which require outputs to be like inputs and thereby act to prevent the neutralization of contrasts. Thus, with the ranking shown in (2), mid vowels are generally permitted, because IDENT[Vht] dominates *MIDV. But in stressed syllables, mid vowels are banned, given the ranking of * MiDV/ó over the stressed syllable-specific faithfulness constraint IDENT[Vht]/o and the general constraint IDENT[Vht].

The problem here is that languages with contrastive mid vowels only in unstressed syllables are in fact unattested. More generally, it is a characteristic of featural positional neutralization effects (such as the neutralization of mid vowels) that they target weak positions, not strong positions (see, e.g., Steriade 1993, 1995 and Beckman 1998 for discussion). So constraints such as *MIDV/ó must not be part of the universal set of constraints. ${ }^{3}$ However, once the formal mechanism of relativizing markedness constraints to strong positions is included in the theory - and such a mechanism is necessary, given the existence of the M/str constraints in (1) - then without any further restrictions, the option of forming an $\mathbf{M} / \mathbf{s t r}$ counterpart is open to all markedness constraints.

This dissertation develops a theory of $\mathbf{M} / \mathbf{s t r}$ constraints that accounts for why some, but not all, of the formally possible $\mathbf{M} / \mathbf{s t r}$ constraints are legitimate, attested constraints. The proposal is outlined in the following section.

\subsubsection{The proposal: Substantive grounding through constraint filters}

What sets attested $\mathbf{M} / \mathbf{s t r}$ constraints like those in (1) apart from unattested and problematic M/str constraints like the putative *MIDV/ó in (2) is their relationship to perceptual prominence. It is proposed here that legitimate $\mathbf{M} / \mathbf{s t r}$ constraints are all strong position-specific versions of augmentation constraints, that is, markedness constraints that require the presence of perceptually prominent

3. Not every ranking of a constraint set that includes * MIDV/ó produces a grammar that allows "reverse positional neutralization" as in (2). But crucially, some such rankings do lead to unattested grammars. Since there is no principled way to prohibit the problem rankings, *MIDV/ó must not be allowed to exist. 
properties such as syllable weight, stress, high tone, high-sonority nuclei, and lowsonority onsets. ${ }^{4}$

This substantively based restriction on $\mathbf{M} / \mathbf{s t r}$ constraints is implemented as a constraint filter in the Schema/Filter model of CoN: the Prominence Condition (§1.2.2.1). Likewise, the additional restriction noted in $\S 1.1$ that holds of $\mathbf{M} / \mathbf{s t r}$ constraints for psycholinguistically strong positions-positional augmentation constraints for these positions are banned if their satisfaction would impede earlystage word recognition, a domain in which psycholinguistically strong positions play a special role-is implemented as another filter: the Segmental Contrast Condition (§1.2.2.2).

\subsubsection{The Prominence Condition}

The requirement that all $\mathbf{M} / \mathbf{s t r}$ constraints must be positional augmentation constraints-constraints that call for the presence of perceptually prominent properties in the strong positions that they target-is enforced by the Prominence Condition, a constraint filter that helps to determine the composition of CON by excluding non-prominence-enhancing $\mathbf{M} / \mathbf{s t r}$ constraints. (For discussion of what it means for a constraint to enhance perceptual prominence, see §2.3.1.)

\section{Prominence Condition}

Markedness constraints specific to strong positions are included in CON only if the general markedness constraints from which they are built call for the presence of perceptually prominent properties.

Essentially, an M/str constraint passes the Prominence Condition if output candidates that satisfy the constraint are judged by the perceptual system to be more prominent - to elicit a perceptual response of greater magnitude - than candidates that violate the constraint. However, the specific type of perceptual prominence demanded by a particular $\mathbf{M} / \mathbf{s t r}$ constraint need not be directly related to the intrinsic salience of the given strong position for the $\mathbf{M} / \mathbf{s t r}$ constraint to pass this filter $(\S 2.3 .3, \S 2.4 .2 .2)$.

Discussion of the Prominence Condition and its role as a constraint filter, and evidence that the predictions of this model are correct in that the empirically

4. On the presence of an onset, or of a specifically low-sonority onset, as a perceptually prominent property for a syllable, see $\$ 2.3 .2 .3$. 
attested $\mathbf{M} / \mathbf{s t r}$ constraints do all qualify as prominence-enhancing constraints, are given in Chapter 2.

\subsubsection{The Segmental Contrast Condition}

Although it is a fundamental requirement that all $\mathbf{M} / \mathbf{s t r}$ constraints must be prominence-enhancing, as enforced by the Prominence Condition, this is not the only requirement that holds of $\mathbf{M} / \mathbf{s t r}$ constraints. Positions that are strong for phonetic reasons are eligible for any kind of positional augmentation constraint, as seen most strikingly in the wide variety of augmentation phenomena observed in stressed syllables (§3.2). However, positions that are strong for psycholinguistic reasons are generally not eligible for positional augmentation constraints that manipulate segmental contrasts (i.e., vocalic or consonantal features; one important exception is discussed below).

Chapter 4 proposes that the defining characteristic of a psycholinguistically strong position is that it be important in early-stage word recognition ( $\$ 4.3 .1)$; the psycholinguistically strong positions initial syllable and root both meet this criterion ( $\S 4.3 .2-3)$. It has been argued by Nooteboom (1981), L. Taft (1984), and others that having a large number of phonological contrasts in positions that are important in early-stage word recognition helps make the task of recognizing words more efficient, because it divides the lexical search space into smaller partitions ( $\$ 4.3 .5)$. This substantive consideration is the reason for the limited positional augmentation possibilities in psycholinguistically strong positions. While augmentation constraints enforce the presence of properties that help make strong positions more perceptually salient, the consequence of satisfying an $\mathbf{M} / \mathbf{s t r}$ constraint is in fact the neutralization of a potential contrast (i.e., in favor of the more perceptually prominent member of the opposition). The added benefit of perceptual prominence that a psycholinguistically strong position would gain from augmentation is simply not enough to outweigh the adverse effects on word recognition of contrast neutralization in this position. Therefore, augmentation effects for these positions are essentially limited to non-segmental properties, such as stress, because prosodic features like this have been shown not to be relevant for early-stage word recognition in the way that segmental contrasts are (see $\S 4.3 .4)$.

While in general there are no augmentation phenomena for psycholinguistically strong positions that affect segmental contrasts, there is one exception to this generalization. The initial syllable is subject to positional augmentation effects involving syllable onsets-ONSET $/ \sigma_{1}$, which requires the presence of a syllable onset in the initial syllable $(\S 4.2 .1 .1)$, and the $\left[{ }^{*} \mathrm{ONSET} / \mathrm{X}\right] / \sigma_{1}$ subhierarchy, which favors low-sonority onsets in this position (\$4.2.1.2). This apparent exception to the 
general ban on segmental-contrast $\mathbf{M} / \mathbf{s t r}$ constraints for psycholinguistically strong positions has a substantive basis as well. As discussed in more detail in Chapter 4, the task of word "segmentation" in spoken-language processing, i.e., locating word boundaries in running speech, is difficult. Thus, enhancing the salience of the initial edge of a word through augmentation processes that affect the onsets of initial syllables is actually helpful in processing.

The two substantive factors that determine possible positional augmentation constraints for psycholinguistically strong positions ( $\Psi_{s t r}$ ), abbreviated $\mathbf{M} / \Psi$ str, are incorporated into the Schema/Filter model by means of another constraint filter, the Segmental Contrast Condition.

\section{Segmental Contrast Condition}

If a constraint is of the form $M / \Psi$ str, then it must meet one of the following two conditions:

I. Satisfaction of the $\mathbf{M}$ constraint from which the $\mathbf{M} / \Psi$ str constraint is built does not alter features that are distinguished in early-stage word recognition.

or

II. $\Psi$ str is $\sigma_{1}$, and satisfaction of the $M / \Psi$ str constraint serves to demarcate the left edge of $\sigma_{1}$.

In brief, condition (I) of the Segmental Contrast Condition allows an $\mathbf{M} / \Psi$ str constraint to be included in CON if its winning and losing candidates (that are otherwise identical) activate the same set of lexical entries; this allows for constraints like HAVESTRESS/Root, since stress placement is not directly relevant in early-stage word recognition, but bans constraints like [ $\left.{ }^{*} \mathrm{PEAK} / \mathrm{X}\right] /$ Root, which would call for high-sonority syllable nuclei in roots, thus neutralizing certain vowelfeature contrasts. Condition (II) allows an $\mathbf{M} / \Psi$ str constraint to be included in CON if it affects the left edge of the initial syllable, as described above. Since the Segmental Contrast Condition is a disjunction, a given $M / \Psi$ str constraint need only pass one of (I) and (II) to be eligible for inclusion in CON. Thus, this filter models the two ways in which substantive considerations affect the inventory of possible $\mathbf{M} / \Psi$ str constraints.

The difference between phonetically and psycholinguistically strong positions, the importance of psycholinguistically strong positions for early-stage word recognition, and the Segmental Contrast Condition are discussed in \$2.4. 
Psycholinguistic evidence supporting these proposals is reviewed in $\S 4.3$, where more detailed justification is also given for the particular formulation of the Segmental Contrast Condition in (4).

\subsubsection{The Schema/Filter model of CON}

The two filters described above, the Prominence Condition and the Segmental Contrast Condition, are proposed as part of a model of CON that allows for free constraint construction from general constraint schemas, like IDENT, ALIGN, or C/str (a constraint schema that relativizes a constraint to a strong position), but also includes substantive restrictions on the phonological system in the form of constraint filters. This model, developed in $\S 2.2$, is summarized in the diagram in (5) below.

The Schema/Filter model of CON

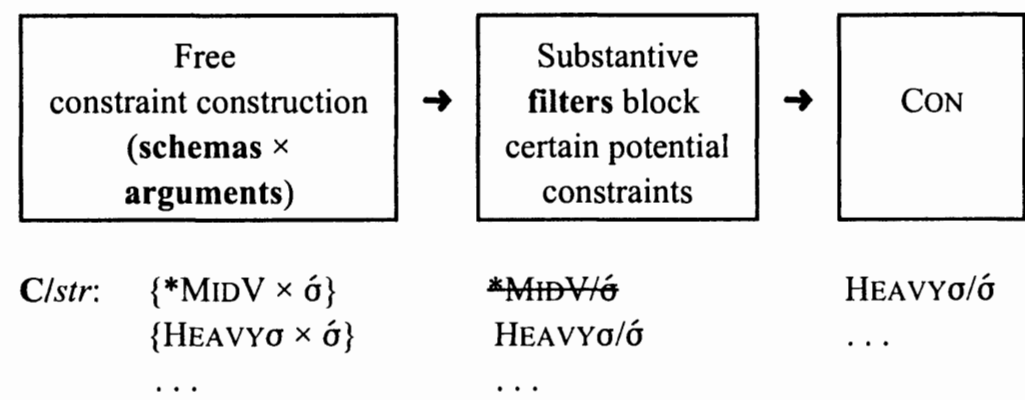

The M/str constraints *MIDV/ó and HEAVYo/ó shown in (5) are both formally possible constraints, since the combination of any constraint with a strong position like ó is a legitimate operation in the constraint-construction module. However, the Prominence Condition will pass only HEAVYo/ó, ruling out *MIDV/ó, since only the former is a prominence-enhancing markedness constraint.

Including constraint filters in the model allows domains external to the formal phonology, such as articulation, perception, and processing, to impose substantive considerations that have a fundamental impact on the contents of the universal constraint set CON, while the constraints themselves remain formal objects, formally constructed. 


\subsection{Strong and weak positions in phonological analysis}

There is one final topic to be addressed before this introductory chapter is concluded: the theoretical background ${ }^{5}$ that is important for understanding the questions examined in this dissertation. The crucial points are evidence for the phonological distinction between strong and weak positions $(\S 1.3 .1)$ and justification for choosing $\mathbf{M} / \mathbf{s t r}$ constraints over $\mathbf{F} / \mathbf{w k}$ constraints (faithfulness constraints for weak positions), a logically possible alternative approach to augmentation constraints on strong positions ( $(1.3 .2)$.

\subsubsection{Phonological evidence for strong positions}

The distinction between strong and weak positions has a long history in modern phonological investigation. Different behavior in strong and weak positions was originally identified in the context of positional neutralization, a phenomenon in which typologically marked structure is tolerated in certain ("strong") positions but neutralized in other ("weak") positions. ${ }^{6}$

Trubetzkoy (1939:235-6) recognizes a phenomenon that he calls "structurally conditioned" neutralization, in which neutralization occurs only in specific positions in the word, because other positions form a "phonological peak" in the word that is able to resist neutralization. To paraphrase Trubetzkoy's (1939) discussion slightly, he proposes two classes of such peaks: first, the location of stress or accent, whether contrastive or fixed, and second, word edges, as opposed to medial positions.

5. Familiarity with the basic framework of Optimality Theory (Prince \& Smolensky 1993; McCarthy \& Prince 1993ab) and the Correspondence Theory implementation of faithfulness (McCarthy \& Prince 1995) is assumed.

6. Strictly speaking, the kinds of phonological processes that are the main topic of this dissertation-prominence-enhancing processes that affect strong positions exclusively - could also be called "positional neutralization," since they cause strong positions to have certain (prominent) properties, thereby neutralizing a potential contrast between the presence and the absence of the property in question. However, the discussion here will follow the traditional use of the term positional neutralization to refer to the types of featural neutralization-neutralization to the unmarked value, with no particular connection to prominence-that affect weak positions exclusively. Processes that affect strong positions exclusively, argued here always to be prominence-enhancing, will be called positional augmentation. 
Subsequent work on positional neutralization has increased the inventory of strong positions. For example, Steriade (1993) recognizes, in addition to initial and final syllables and stressed syllables, released consonants and long vowels. Steriade $(1993,1995,1997)$ also makes the important point that in some cases, the identity of the features whose neutralization can be resisted by a particular strong position depends on the phonetic characteristics of the strong position (for more on this and related matters, see §2.4). McCarthy \& Prince (1995) and Alderete (1999b, 2001) present evidence for the importance of the morphological root (as opposed to affixes) as a strong position. Smith $(1998,1999,2001)$ proposes that the noun can also be considered a strong position with respect to positional neutralization effects, perhaps because the noun is more canonically rootlike-more likely to be a free form - than the verb. Discussion of differences between strong and weak positions can also be found in, for example, Vennemann (1972), Hooper (1976), Jun (1995), Padgett (1995), Zoll (1996, 1997a, 1998), and Lombardi (1999).

Beckman (1997, 1998; see also Casali 1996, 1997) proposes that a particular position may qualify for special status as a strong position, and therefore have the potential to resist positional neutralization effects, for one of two reasons. Either, as in Steriade's $(1993,1995,1997)$ proposals, the position has special phonetic salience, or else the position has a special role in psycholinguistic processing. That is, it is special status outside the domain of phonology proper that gives rise to special phonological status as a strong position.

The strong positions to be examined in this dissertation are listed in (6), grouped, according to Beckman's (1998) terminology, into phonetically and psycholinguistically strong positions (see also $§ 2.3 .3, \S 2.4 .2){ }^{7}$

7. Not all of the strong positions that have been proposed in the literature are given equal attention here. Word-final position is explicitly recognized as a strong position in some accounts of positional neutralization, such as Trubetzkoy (1939) and Steriade (1993); see also Hyman's (1998) discussion of the word-medial "prosodic trough" in Bantu languages, where he shows that possible phonological contrasts are more limited in medial than in final position, and Zhang (2000), who argues that contour tones can be licensed specifically in final syllables. However, investigation to date has not found any convincing cases of augmentation in final position. Since positional augmentation is the focus of this dissertation, this position is not further considered here.

Other strong positions proposed in the phonological literature for which augmentation constraints can plausibly be identified are the noun and certain prosodic heads (other than the main-stress syllable, which is extensively discussed in $\$ 3.2$ ). One possible case of noun augmentation is discussed in $\$ 4.2 .2 .2$. Systematic examination of augmentation in prosodic heads other than the main-stress syllable (head of the prosodic word, PrWd) is a topic for future investigation, although prosodic heads at several levels do appear to have the status 
(6) Strong positions

(a) Phonetically strong positions

Stressed syllable

Onset/released consonant ${ }^{8}$

Long vowel (b) Psycholinguistically strong positions

Initial syllable

Root

$\S 2.4$ shows that this distinction between the two classes of strong positions is a fundamental division that affects the ways in which constraints, both markedness and faithfulness, can be relativized to strong positions. The distinction between phonetically and psycholinguistically strong positions is therefore relevant for both kinds of position-sensitive phenomena, positional neutralization and positional augmentation.

In summary, investigation of positional neutralization has shown that phonologically strong and weak positions must be distinguished. The positional augmentation phenomena treated in this dissertation provide another class of cases in which strong and weak positions show distinct patterns of phonological behavior. Further discussion of positional neutralization, and its relationship to the positional augmentation phenomena under investigation here, is provided in Chapter 5 . $\$ 5.2$ reviews the three approaches that have been taken in OT toward positional neutralization (positional faithfulness constraints, positional featural markedness constraints, and COINCIDE constraints), showing that no account of positional neutralization can be used "as-is" to account for positional augmentation effects as well. $\S 5.3$ argues that positional augmentation is a phenomenon that is empirically distinct from positional neutralization, so it is not surprising that the two require distinct formal treatments. $§ 5.3$ also shows that the factorial typology of a system that includes both positional neutralization and positional augmentation constraints is consistent with observed empirical patterns.

8. This strong position will be referred to as "onset" for expository convenience, but as argued by, e.g., Kingston (1985, 1990), Lombardi (1991, 1999), and Padgett (1995), it is best defined as a consonant that is released. (See also Steriade 1993, 1995, 1997 for discussion of this position.) 


\subsubsection{Positional augmentation constraints as $\mathrm{M} / \mathrm{str}$, not " $\mathrm{F} / \mathrm{wk}$ "}

This dissertation examines a particular set of phenomena in which strong and weak positions are distinguished: phonological requirements that are enforced of strong positions, but not of weak positions. This section demonstrates that such positionspecific requirements can be enforced within OT only through constraints that are themselves relativized to positions, and defends the choice of $\mathbf{M} / \mathbf{s t r}$ constraints (markedness constraints relativized to strong positions) over $\mathbf{F} / \mathbf{w k}$ constraints (faithfulness constraints relativized to weak positions) for this purpose.

Fundamental to the nature of phonological analysis in OT is the interaction between markedness (M) and faithfulness (F) constraints (see also §1.2.1). F constraints demand the preservation of features, segments, and other phonological structures or configurations in the mapping from input to output forms (likewise for correspondence relations other than input-output). Thus, if a given $\mathbf{F}$ constraint is not dominated by any conflicting constraints, there will be a contrast in the language involving the phonological feature or structure to which that $\mathbf{F}$ constraint makes reference. The surface phonological contrast comes about because inputs having the feature or structure in question will correspond to outputs that also have it, whereas inputs lacking the feature or structure will correspond to outputs that also lack it.

$\mathbf{M}$ constraints, on the other hand, require output forms to avoid dispreferred phonological features and structures (dispreferred for, e.g., articulatory or perceptual reasons). So if an $\mathbf{M}$ constraint banning a particular phonological feature or structure dominates an $\mathbf{F}$ constraint that would otherwise protect the feature or structure in question, then output forms in the language will predictably lack that feature or structure, which means that it does not form the basis of a phonological contrast.

For example, consider the following commonly invoked constraints (given informal characterizations for now; see also §2.3.2.3 concerning ONSET).

(a) M: ONSET

(b) F: DEP-SEG
Syllables begin with consonants

(Prince \& Smolensky 1993, after Itô 1986, 1989)

Epenthesis is prohibited

(McCarthy \& Prince 1995)

In a language where ONSET dominates DEP-SEG $(\mathbf{M}>\mathbf{F})$, all syllables will have onsets, so the language will have no contrast between $\mathrm{C}$-initial and $\mathrm{V}$-initial syllables (8a). Conversely, in a language where DEP-SEG dominates ONSET (F $>>$ 
M), there will be a surface contrast between output forms that have V-initial syllables and those that do not $(8 b) .{ }^{9}$

$\mathbf{M}, \mathbf{F}$, and phonological contrast

(a) $\mathbf{M} \gg$ F: no contrast; $/ \mathrm{V} / \rightarrow[\mathrm{CV}]$ and $/ \mathrm{CV} / \rightarrow[\mathrm{CV}]$

\begin{tabular}{|c||c|c|}
\hline (i) $/ \mathrm{V} /$ & ONSET & DEP-SEG \\
\hline \hline a. V & $* !$ & \\
\hline b. CV & & $*$ \\
\hline (ii) $/ \mathrm{CV} /$ & ONSET & DEP-SEG \\
\hline \hline a. CV & & \\
\hline b. V & $* !$ & \\
\hline
\end{tabular}

(b) $\mathbf{F} \gg$ M: contrast persists; $/ \mathrm{V} / \rightarrow[\mathrm{V}]$ and $/ \mathrm{CV} / \rightarrow[\mathrm{CV}]$

\begin{tabular}{|c||c|c|}
\hline (i) $/ \mathrm{V} /$ & DEP-SEG & ONSET \\
\hline \hline a. V & & $*$ \\
\hline b. CV & $* !$ & \\
\hline (ii) $/ \mathrm{CV} /$ & DEP-SEG & ONSET \\
\hline \hline a. CV & & \\
\hline b. V & & $* !$ \\
\hline
\end{tabular}

9. The purpose of this example is not to present a detailed discussion of syllable inventory typology (for which see, e.g., Prince \& Smolensky 1993), but rather to illustrate the interaction of $\mathbf{M}$ and $\mathbf{F}$ constraints with respect to phonological contrast. Therefore, it has been deliberately oversimplified for expositional clarity. For example, the ranking of the F constraint MAX-SEG, which militates against segmental deletion, is also relevant in both model languages shown in (8). To have a language as in (8a) that avoids onsetless syllables by epenthesizing onsets (rather than by deleting potentially onsetless vowels), MAX-SEG must dominate DEP-SEG. To have a language as in (8b) that preserves onsetless syllables (again, rather than one that eliminates them through vowel deletion), MAX-SEG must dominate ONSET. 
When a particular phonological contrast is attested in a language only in certain structural positions, however, general $\mathbf{M}$ and $\mathbf{F}$ constraints alone are insufficient to account for the pattern. One relevant example would be a language in which syllables in some strong position str always have onsets, but syllables outside str may have onsets or not. In other words, syllables in str have no contrast between V and CV syllables (as in (8a)), but syllables outside str do have that contrast (as in (8b)). Languages of this type include Dutch, which requires onsets specifically in stressed syllables (\$3.2.2.1), and Arapaho, which requires onsets specifically in initial syllables ( $\$ 4.2 .1 .1)$.

To account for a phonological contrast that surfaces only outside a strong position $s t r$, it is necessary to have a ranking of the form $\mathbf{F} \gg \mathbf{M}$ outside $s t r$ but $\mathbf{M}$ > $\mathbf{F}$ inside str. Formally, there are three ways in which this sort of differential ranking can be accomplished. One option is literally to recognize distinct constraint rankings that operate inside and outside the position in question. However, choosing this option would force us to abandon the hypothesis that there is a single, consistent constraint ranking for an entire language; this option will therefore not be further considered here. (See Fukazawa, Kitahara, and Ota (1998) for arguments in favor of maintaining a single total ranking for the grammar of a language. ${ }^{10}$ )

The two remaining options are to invoke a version of the $\mathbf{M}$ constraint that is specific to the strong position (M/str(ong)), or to invoke a version of the $\mathbf{F}$ constraint that is specific to the complement of the strong position (F/w(ea)k). Constraints that make reference to particular positions have often been proposed in the literature in situations of positional neutralization (contrast neutralization in weak positions); see Chapter 5 for a discussion of such proposals and the formal nature of the positional constraints involved. The crucial insight behind the use of positional constraints is that they assess violations only when the state of affairs that they prohibit occurs in the position to which they are relativized; in effect, they ignore anything that happens outside that position. ${ }^{11}$

The first of these positional-constraint strategies, the one using $\mathbf{M} / \mathbf{s t r}$ constraints, is the approach actually adopted here. It solves the problem of strong

10. Anttila (2002) takes the opposite position from Fukazawa, Kitahara, and Ota (1998), arguing in favor of distinct subgrammars (separate rankings) for, e.g., different sets of lexical items within a language. However, such a proposal is not applicable for the majority of the cases considered in this dissertation, since the differing phonological behavior occurs, not in distinct lexical items, but in distinct structural positions within individual lexical items.

11. One class of position-sensitive constraints, namely CoINCIDE constraints (Zoll 1996, 1997a, 1998), is somewhat different in this respect. This is because CoINCIDE constraints are not themselves relativized to a position. Instead, they take a strong position as one of the elements over which they quantify in computing violations. See $§ 5.2 .3$ for discussion. 
position-specific markedness effects by including in the grammar versions of $\mathbf{M}$ constraints that make specific reference to individual strong positions, such as ONSET $/ \sigma$ for Dutch or ONSET $/ \sigma_{1}$ for Arapaho.

Thus, the relevant ranking for Arapaho, which requires onsets specifically in initial syllables, is as follows (see also (1d) above and $§ 4.2 .1 .1$ ).

(9) Onsets required specifically in initial syllables (data from Salzmann 1956; syllabification according to Salzmann's description)

(a) Medial syllables need not have onsets

\begin{tabular}{|c||c|c|c|c|}
\hline /XOOó/ & ONSET/ 11 & $\begin{array}{c}\text { DEP- } \\
\text { SEG }\end{array}$ & $\begin{array}{c}\text { MAX- } \\
\text { SEG }\end{array}$ & ONSET \\
\hline \hline a. xoo.ó & & & & $*$ \\
\hline b. xoo.Có & & $* !$ & & \\
\hline c. $x 00$ & & & $* !$ & \\
\hline
\end{tabular}

(b) Initial syllables must have onsets (hypothetical input)

\begin{tabular}{|c|c|c|c|c|}
\hline /owo?/ & ONSET $/ \sigma_{1}$ & $\begin{array}{l}\text { DEP- } \\
\text { SEG }\end{array}$ & $\begin{array}{c}\text { MAX- } \\
\text { SEG }\end{array}$ & ONSET \\
\hline (必) a. Co.wo? & & * & & \\
\hline (果) b. wo? & & & * & 63 \\
\hline c. o.wo? & $* !$ & & & $*$ \\
\hline
\end{tabular}

In medial syllables, high-ranking ONSET/ $\sigma_{1}$ is by definition not applicable (is vacuously satisfied), so the relevant constraints are DEP-SEG, MAX-SEG $\gg>$ ONSET; that is, $\mathbf{F} \gg \mathbf{M}$. This is the ranking for contrast, so non-initial syllables have a contrast between syllables without onsets (9a) and syllables with onsets (e.g., [no.wo?] in (1d) above).

The lack of contrast specifically in initial syllables is enforced by the high rank of ONSET $/ \sigma_{1}$. This position-specific markedness constraint dominates at least one of the faithfulness constraints MAX-SEG or DEP-SEG, so that within the strong position initial syllable, the relevant ranking is $\mathbf{M}(/ \mathbf{s t r})>\mathbf{F}$, the ranking for 
neutralization. Potential V-initial input forms therefore surface unfaithfully, to be C-initial (9b).

It should be noted here that there are no examples in Salzmann (1956) showing overt alternations, which would indicate specifically what the grammar of Arapaho actually does when confronted with an onsetless syllable in word-initial position - that is, whether the input /owo?/ would actually map to [Co.wo?] (9b, candidate $a$ ) or to [Wo?] (9b, candidate $b$ ) (or even to something else, such as the metathesis candidate [wo.0?]). This is why the two unfaithful candidates in tableau (9b) are both labeled with a parenthesized '( > F ranking does exist for initial syllables, since initial syllables show no contrast between $\mathrm{V}$ and $\mathrm{CV}$ syllables. ${ }^{12}$ The $\sigma_{1}$-specific constraint ONSET $/ \sigma_{1}$ clearly dominates at least one faithfulness constraint, whichever particular $\mathbf{F}$ constraint that may be.

Thus, under the approach adopted here, phonological requirements that hold specifically within strong positions are analyzed by means of $\mathbf{M} / \mathbf{s t r}$ constraints ranked as shown in (10).

(10) Positional augmentation ranking with $\mathbf{M} / \mathbf{s t r}$ constraints

$$
\begin{aligned}
& \mathbf{M} / \mathbf{s t r} \gg \mathbf{F} \quad \gg \quad M \\
& \left.L_{\mathbf{M}} \gg \mathbf{F} \text { in } s t r\right\rfloor \quad \text { Result: No contrast in str } \\
& \text { LF }>>\text { M outside } s t r\lrcorner \quad \text { Result: Contrast outside } s t r
\end{aligned}
$$

The other ranking permutations of these three general constraint types produce typologically attested patterns as well. ${ }^{13}$

12. By the OT principle of richness of the base (Prince \& Smolensky 1993), according to which there can be no language-specific restrictions on input forms, the grammar of Arapaho must map any universally possible input shape (including a word that starts with a vowel) to an output that is compatible with the surface phonotactics of Arapaho. In other words, the fact that Arapaho never has vowel-initial words means that its constraint ranking must be one that would force a vowel-initial word to surface unfaithfully (with an initial onset) if the grammar were given such an input. This is why we must conclude that ONSET $/ \sigma_{1}$ dominates at least one faithfulness constraint-so that even a hypothetical input like /owo?/ will not surface with a word-initial vowel.

13. On cases of complementary distribution, where different allophones appear inside and outside of a particular strong position, see $\S 5.3$. 
(11) Other rankings for $\mathbf{M} / \mathbf{s t r}$ constraints, and predicted outcomes

(a) $\mathbf{M} \gg \mathbf{F}$ (M/str rank irrelevant) Result: No contrast in any position

(b) $\mathbf{F} \gg\{\mathbf{M}, \mathbf{M} / \mathbf{s t r}\} \quad$ Result: Contrast in all positions

The second way to use position-specific constraints in analyzing phonological processes that specifically affect material in strong positions would be to invoke F/wk constraints, that is, faithfulness constraints that make specific reference to weak positions. This alternative is equivalent in its broad typological predictions to the $\mathbf{M} / \mathbf{s t r}$ approach, but it is conceptually less attractive.

Like the $\mathbf{M} / \mathbf{s t r}$ approach, the $\mathbf{F} / \mathbf{w k}$ approach to positional augmentation formally allows for a differential ranking relationship inside and outside a strong position. Given a constraint set that includes general $\mathbf{M}$ constraints, general $\mathbf{F}$ constraints, and $\mathbf{F} / \mathbf{w k}$ constraints, the following ranking generates a language with positional augmentation (contrast neutralization in strong positions only); comparison with the $\mathbf{M} / \mathbf{s t r}$ approach in (10) above shows that the general pattern produced is equivalent.

(12) Positional augmentation ranking with $\mathbf{F} / \mathbf{w k}$ constraints

$$
\begin{aligned}
& \text { F/wk } \gg \text { M }>\text { F } \\
& \text { LM } \gg \text { > F in } s t r\rfloor \quad \text { Result: No contrast in str } \\
& \text { LF }>>\text { M outside } s t r\rfloor \quad \text { Result: Contrast outside } s t r
\end{aligned}
$$

Furthermore, as seen in (13), the permuted rankings of the general constraint types shown in (12) produce the same set of typologically attested language types as the $\mathbf{M} /$ str system (compare (11)).

(13) Other rankings for $\mathbf{F} / \mathbf{w k}$ constraints, and predicted outcomes
(a) $\mathbf{M} \gg\{$ F, F/wk $\}$
Result: No contrast in any position
(b) $\mathbf{F}>>\mathbf{M}(\mathbf{F} / \mathbf{w k}$ rank irrelevant $) \quad$ Result: Contrast in all positions

Nevertheless, there is an important difference between the two approaches that becomes apparent as soon as the nature of the individual positional constraints required under each system is examined. Specifically, in order for $\mathbf{F}$ constraints to be relativized to weak positions, those weak positions must be formally identifiable 
by the part of the grammar that is responsible for producing position-specific constraints. In some cases, weak positions are identifiable in their own right: the weak counterpart to the strong position root would be the affix (morphologically identified); the weak counterpart to the strong position stressed syllable would be the unstressed syllable (metrically/prosodically identified).

However, for other strong positions, the corresponding weak position is not something that can be identified except as the complement of the strong position. One such case is the "non-initial syllable," which would have to be identified by the position-specific $\mathbf{F}$ constraint in an F/wk-based approach to the Arapaho facts discussed above. The "position" non-initial syllable can only be identified as "any syllable that is not the initial syllable." In other words, it is more straightforward and conceptually appealing to allow the grammar access to strong positions which it then uses to form positional constraints, than to suppose that the grammar first locates strong positions, then identifies their complements, and finally forms positional constraints with reference to the complements of the strong positions.

For this reason, it is proposed here that position-specific constraints can only refer to strong positions, never to weak positions. Under this principle, F/wk constraints are simply not available as a way to account for phonological requirements that hold specifically of strong positions. (On the implications of this proposal for theories of classical positional neutralization, see Chapter 5).

It is also important to recognize that choosing an $\mathbf{F} / \mathbf{w k}$ approach rather than an $\mathbf{M} / \mathbf{s t r}$ approach to positional augmentation phenomena would not avoid the fundamental problem of excluding empirically unattested types of strong positionspecific neutralization (see $\S 1.2 .1$ above). It would still be necessary for the model to allow some $\mathbf{F} / \mathbf{w k}$ constraints while excluding others. For example, the $\mathbf{F} / \mathbf{w k}$

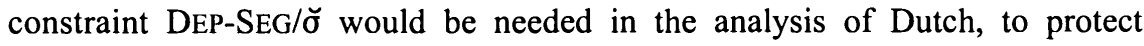
unstressed syllables from the epenthesis of onset consonants that takes place specifically in stressed syllables (\$3.2.2.1). But allowing featural F/wk constraints

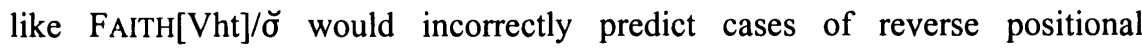
neutralization - the neutralization of featural contrasts unrelated to the enhancement of perceptual prominence-when such constraints were ranked as in (14) (compare the $\mathbf{M} / \mathbf{s t r}$ case in (2)). 


\section{Problematic F/wk constraints}

IDENT[Vht] $/ \breve{\sigma}>>*$ MIDV $>$ IDENT[Vht] $\rightarrow$ mid V in $\breve{~ o n l y ~(u n a t t e s t e d) ~}$

\begin{tabular}{|r||c|c|c|}
\hline Input: /tépo/ & IDENT[Vht]/ŏ & $*$ MIDV & IDENT[Vht] \\
\hline \hline a. tépo & & $* * !$ & \\
\hline b. típo & & $*$ & $*$ \\
\hline c. tipu & $* !$ & & $* *$ \\
\hline
\end{tabular}

Thus, a system using $\mathbf{F} / \mathbf{w k}$ constraints instead of $\mathbf{M} / \mathbf{s t r}$ constraints would still require a theory of possible and impossible $\mathbf{F} / \mathbf{w k}$ constraints, to explain why, e.g., DEP-SEG/б̆ would be a legitimate F/wk constraint but IDENT[Vht]/б̆ would not. The solution developed here, that phonological phenomena specifically targeting strong positions can only be those that give the strong positions greater perceptual prominence, would still have to be part of the theory of possible F/wk constraints, just as it is incorporated in the current model of $\mathbf{M} / \mathbf{s t r}$ constraints in the form of the Prominence Condition. However, under the F/wk approach, the positional constraints themselves would actually refer neither to the strong positions, nor to the prominent properties that are, descriptively speaking, the "goal" of the strong position-specific phonological processes in question. It would therefore be more difficult to propose a substantively grounded filter that could rule out the problematic $\mathbf{F} / \mathbf{w k}$ constraints, because constraints of that type would be less directly related to perceptual prominence and strong positions.

In summary, under the $\mathbf{M} / \mathbf{s t r}$ approach to positional augmentation, the grammar need only make reference to strong positions, rather than having to make reference indirectly to the complements of strong positions. Furthermore, a central component of the theory of positional augmentation effects-that phonological processes may exclusively target strong positions only when those processes result in greater perceptual prominence for the strong positions-is more straightforwardly modeled as a constraint filter under the M/str approach. Therefore, this is the approach that is implemented here.

\subsection{Outline of the dissertation}

The dissertation is structured as follows. Chapter 2 presents the theoretical model of positional augmentation: the Prominence Condition and the Segmental Contrast 
Condition, which restrict the inventory of $\mathbf{M} / \mathbf{s t r}$ constraints, in the context of the Schema/Filter model of CON. This chapter shows that the predictions of the model match the patterns found in empirical investigations of positional augmentation. Namely, the markedness constraints that have empirically attested $\mathbf{M} / \mathbf{s t r}$ counterparts are all shown to be augmentation (prominence-enhancing) constraints, so the Prominence Condition correctly predicts that they can have $\mathbf{M} / \mathbf{s t r}$ versions. Also, the importance of psycholinguistically strong positions ( $\Psi_{s t r}$ ) for early-stage word recognition is shown to further restrict the set of possible $\mathbf{M} / \Psi$ str constraints, as predicted by the Segmental Contrast Condition.

Chapters 3 and 4 present case studies exemplifying $\mathbf{M} / \mathbf{s t r}$ constraints for phonetically and psycholinguistically strong positions respectively. Chapter 4 also addresses additional matters pertaining to positional augmentation in psycholinguistically strong positions, including a review of evidence from psycholinguistic studies that justifies the particular formulation of the Segmental Contrast Condition given here.

Chapter 5 reviews OT proposals that address the special resistance of strong positions to positional neutralization, showing that any theory of phonological requirements for strong positions must be distinct from extant theories of positional licensing or resistance to featural neutralization in those positions.

Finally, Chapter 6 presents concluding remarks and considers further implications of the proposals developed in the preceding chapters. 


\section{A Theory of \\ Positional Augmentation Constraints}

\subsection{Introduction}

As outlined in Chapter 1, a successful theory of markedness constraints that apply exclusively to phonologically strong positions (M/str constraints) must be able to predict which markedness constraints have $\mathbf{M} / \mathbf{s t r}$ counterparts and which do not. The proposal developed here is that the distinction between legitimate, attested $\mathbf{M} / \mathbf{s t r}$ constraints and problematic, unattested $\mathbf{M} / \mathbf{s t r}$ constraints is made on substantive grounds, involving factors such as perceptual prominence and the way in which certain strong positions are involved in word recognition. As a consequence, a theory of $\mathbf{M} / \mathbf{s t r}$ constraints must be seen in the context of a broader question: how it is that substantive restrictions can affect the phonological system.

This chapter, which lays out the theoretical framework within which the analysis of phonological requirements for strong positions is to be developed, therefore addresses three main topics. First, $\$ 2.2$ presents a general theory of the interaction between substantive grounding and the formal grammar: the Schema/Filter model of the universal constraint set CON. An explicit formal treatment of relativized (position-specific) constraints is also developed, under which the formulation of a relativized constraint such as ONSET/ó is compositionally derived from the formulation of the corresponding general constraint.

The next two sections then apply the framework developed in $\S 2.2$ to the specific case of $\mathbf{M} / \mathbf{s t r}$ constraints. $\S 2.3$ is concerned with the crucial relationship between M/str constraints and perceptual prominence. In $\S 2.3 .1$, the Prominence 
Condition, which screens potential $\mathbf{M} / \mathbf{s t r}$ constraints to ensure that they are built from augmentation (prominence-enhancing) constraints, is implemented as one of the filters in the Schema/Filter model. In $\$ 2.3 .2$, a number of augmentation constraints are examined. For each constraint, the nature of its relationship to perceptual prominence is discussed and an explicit constraint formulation is given. \$2.3.3 enumerates the $\mathbf{M} / \mathbf{s t r}$ versions that are predicted to exist for each augmentation constraint, given the approach to relativized constraints developed in $\S 2.3 .1 .2$ and the size of the element that serves as the focus of the $\mathbf{M}$ constraint in question. The inventory of predicted $\mathbf{M} / \mathbf{s t r}$ constraints is then compared to the inventory of attested $\mathbf{M} / \mathbf{s t r}$ constraints from the case studies in chapters 3 and 4 .

Then, $\$ 2.4$ discusses the substantive pressures that specifically affect

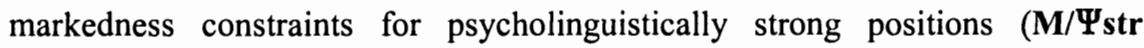
constraints) because these positions derive their special status from their importance in early-stage word recognition. The substantive pressures in question are implemented in the model as a second constraint filter, the Segmental Contrast Condition. This filter disallows even augmentation constraints from being relativized to psycholinguistically strong positions if they would call for excessive neutralization of contrasts that are relevant for early-stage word recognition (§2.4.1). The differences between phonetically and psycholinguistically strong positions, and other domains of phonology in which these differences are potentially relevant, are addressed in $§ 2.4 .2$.

Finally, conclusions and implications are discussed in $§ 2.5$.

\subsection{Formal models of constraints and CON}

Research in the framework of OT attempts to explain phonological phenomena by means of a set of ranked and violable constraints. But in order for phonological analyses to be as constrained, and thus as predictive, as possible, there must be a theory of what constitutes a well-formed or legitimate constraint.

Here, the empirical focus is those phonological requirements that specifically target phonetically and psycholinguistically strong positions; such requirements are to be analyzed by means of $\mathbf{M} / \mathbf{s t r}$ constraints, as outlined in $\S 1.3 .2$. However, a theory of possible and impossible $\mathbf{M} / \mathbf{s t r}$ constraints can only be developed in the context of a general theory of the nature of constraints and the universal constraint set.

This section presents two components of a general theory of constraints. First, the Schema/Filter model of CON, a theory of the influence of substantive considerations on the formal phonological system, is proposed in $§ 2.2 .1$. Then, an 
explicitly compositional treatment of position-specific constraints-in other words, an account of what it means to have constraints that are relativized to particular positions, and how such constraints are related to their context-free counterparts-is presented in \$2.2.2. The Schema/Filter model and the compositional approach to relativized constraints are both integral parts of the theory of possible and impossible $\mathbf{M} / \mathbf{s t r}$ constraints that is developed in later sections of this chapter.

\subsubsection{The Schema/Filter model of CoN}

This subsection presents the Schema/Filter model of the universal constraint set CoN, a model of how substantive considerations affect the nature of the constraint set. For example, as outlined in $\S 1.2$, there is a substantive restriction on $\mathbf{M} / \mathbf{s t r}$ constraints, stated informally as in (1) (see $\$ 2.3 .1$ for a more detailed discussion of this restriction).

\section{The Prominence Condition}

Markedness constraints specific to strong positions are included in CON only if the general markedness constraints from which they are built call for the presence of perceptually prominent properties.

However, an important question arises when a restriction like the Prominence Condition is proposed. How can a statement about which constraints are or are not included in CON be implemented as part of the theory of grammar rather than as an extragrammatical observation?

This question is actually part of a more general problem concerning the nature of CoN. Namely, why do certain constraints exist, when others, although built from the same set of phonological elements combined in the same ways, do not? For example, Eisner (1997) observes that a theory that builds constraints from primitive elements, and is able to generate the familiar constraints ONSET ('Syllables have onsets; syllables are left-aligned with $\mathrm{C}^{\prime}$ ) and NoCoDA ('Syllables lack codas; syllables are right-aligned with $\left.\mathrm{V}^{\prime}\right)$, also generates the converse constraints "NOONSET ('syllables are left-aligned with $\mathrm{V}^{\prime}$ )" and "CODA ('syllables are rightaligned with $C^{\prime}$ )," which are not part of CON (Prince \& Smolensky 1993). Something must ensure that ONSET and NOCODA are existing constraints, while "NOONSET" and "CODA," although they are formally possible constraints, do not in fact exist. Fukazawa \& Lombardi (2000) raise similar questions about the use of constraint conjunction to derive complex constraints. 
Hayes (1999a) considers this problem with respect to articulation-based markedness constraints, ${ }^{1}$ comparing attested and phonetically plausible constraints like *NÇ $(*$ [+nas][-voi]; Pater 1996, 1999) with implausible and unattested constraints like "POSTSONORANT-DEVOICING (*[+son][+voi])." Hayes proposes that all logically possible constraints of this sort, which ban particular features in particular environments, are evaluated by a principle known as Inductive Grounding. Essentially, a constraint passes the inductive-grounding requirement if it partitions the phonetic space into "easy" and "difficult" articulations more accurately than constraints of equal or greater formal simplicity do. ${ }^{*} \mathrm{NC}$ is inductively grounded, while "POSTSONORANT-DEVOICING" is not, so only the former is included in CON.

The Schema/Filter model of CoN developed here expands on Hayes' (1999a) proposal that knowledge of articulatory or perceptual difficulty can be used to distinguish between certain kinds of possible and impossible constraints. The Schema/Filter model is a constraint "metagrammar" consisting of two components: a set of constraint schemas, which are functions that apply to arguments (primitive phonological elements) in order to construct individual constraints; and a set of constraint filters, which make use of articulatory, acoustic, perceptual, and other substantive information to distinguish between legitimate and impossible constraints. The Schema/Filter model is summarized in (2).

The Schema/Filter model of CoN: a constraint metagrammar

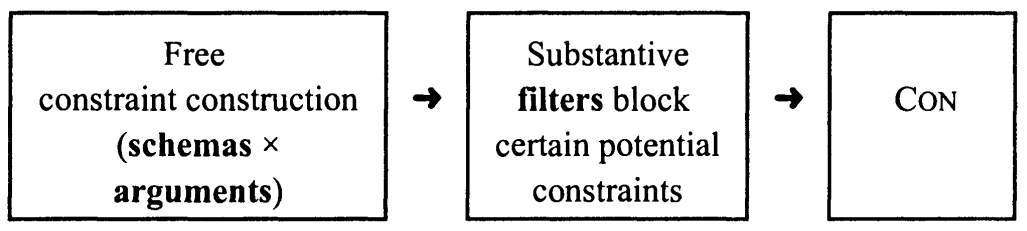

Constraint schemas and constraint filters are discussed in $\S 2.2 .1 .1$ and $§ 2.2 .1 .2$ respectively.

Once the Schema/Filter model has been developed in this section, its relevance to the specific question of $\mathbf{M} / \mathbf{s t r}$ constraints is demonstrated in subsequent sections. Namely, the Prominence Condition (1) is incorporated into the constraint metagrammar as a filter on $\mathbf{M} / \mathbf{s t r}$ constraints (§2.3). Likewise, the Segmental Contrast Condition, which places additional restrictions on augmentation constraints

1. Hayes (1999a) proposes that Inductive Grounding is relevant for constraints that are perceptually grounded, as well as those that are articulatorily grounded. However, the example that he discusses in detail is an articulatory case. 
for psycholinguistically strong positions beyond those imposed by the Prominence Condition, is implemented as a constraint filter on $\mathbf{M} / \Psi$ str constraints (§2.4).

\subsubsection{Constraint schemas}

It would be logically possible to view the universal constraint set CON as an arbitrary collection of unitary, unanalyzable constraints that is supplied by Universal Grammar. However, there are several reasons for preferring a conception of CON in which constraints are constructed from more basic elements. For one thing, constraints that refer to individual morphemes in a language (such as alignment constraints that cause particular morphemes to be prefixes or suffixes) cannot possibly be included in Universal Grammar in their final form, since they include language-specific information. Thus, there must be a process of constraint construction for at least some constraints (as when the universally available Generalized Alignment schema is applied to individual morphemes in a given language; McCarthy \& Prince 1993a). Even many plausibly universal constraint families, such as the IDENT $[F]$ family or the $*[F, G]$ family (where $F$ and $G$ are variables that stand for phonological features), are transparently composed of a general constraint schema applied to a number of elements of a particular type (Smolensky 1995) -in these two cases, features - and furthermore, the formulation of each of the resulting constraints in the family (e.g., ${ }^{*}\left[-\operatorname{son}_{F},{ }^{+v_{0}} i_{G}\right]$ : '[-son $]_{F}$ and $[+ \text { voi }]_{G}$ must not co-occur in the same segment') is completely predictable. To ignore such regularity would be to miss a generalization. Moreover, assuming that the contents of CON are universally supplied as-is would make it more difficult to integrate substantive grounding into the theory of grammar. One could say only that the constraints included in CON happen to be those that are substantively grounded; substantive considerations would play no active role in shaping CON.

For these reasons, the approach to CoN taken here is the Schema/Filter model. As outlined above (see (2)), one component of this model is a constraintconstruction module in which all formally possible constraints are constructed from the combination of a set of constraint schemas, which are modeled as functions, and a set of primitive phonological elements that serve as arguments for the schemas (including a set of strong positions to which general constraints can be relativized). ${ }^{2}$

2. Other proposals to treat the constraint set as a system built up from a set of basic elements include Eisner (1997), who proposes that many OT constraints can be recast in terms of two primitive relations between phonological elements, 'temporally overlaps' and 'does not temporally overlap'; and analyses that view various constraint types as built from 
The concept of a constraint schema has its origin in the Generalized Alignment treatment of alignment constraints (McCarthy \& Prince 1993a), shown in (3). (See also Smolensky 1995 on "parametrized families" of constraints and Suzuki 1998 on a schema for OCP constraints.)

(3) The ALIGN schema (Generalized Alignment; McCarthy \& Prince 1993a:80)

$\operatorname{Align}($ Cat 1, Edge1, Cat2, Edge2)

$\forall$ Cat $1 \exists$ Cat 2 such that Edge 1 of Cat 1 and Edge 2 of Cat 2 coincide

where Cat 1 , Cat $2 \in P C a t \cup M C a t$ (i.e., prosodic and morphosyntactic categories)

Edge1, Edge2 $\in\{\mathrm{R}(\mathrm{ight}), \mathrm{L}(\mathrm{eft})\}$

The ALIGN schema applies to edges and grammatical categories to create individual alignment constraints, such as ALIGN(Root, L, PrWd, L) in (4) (where PrWd = Prosodic Word).

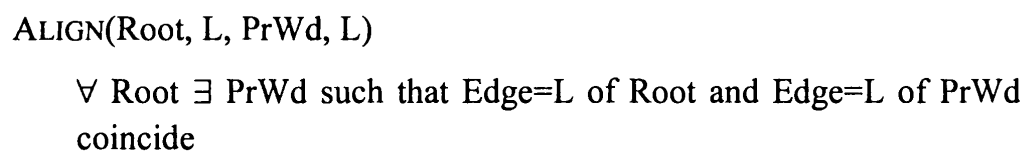

$\forall$ Root $\exists \operatorname{PrWd}$ such that Edge $=\mathrm{L}$ of Root and Edge $=\mathrm{L}$ of PrWd coincide

Note that the formulation of the constraint ALIGN(Root, L, PrWd, L) is completely compositional, given the formulation of the ALIGN schema and the choice of Cat(egorie)s and Edges used in building this particular alignment constraint.

This approach to forming specific constraints out of general constraint types is generalized in the Schema/Filter model, where all constraints are built by applying schemas to arguments. For example, there is an IDENT schema of the form IDENT-Corr[Feat], with the following formulation.

other, simpler constraints through constraint conjunction (including Smolensky 1995, 1997; Zoll 1998). 
(5) The IDENT schema

IDENT-Corr [Feat $] \quad$ If $S_{1}$ and $S_{2}$ are strings related by the correspondence relation ${ }^{3}$ Corr, $\alpha \in \mathrm{S}_{1}, \beta \in \mathrm{S}_{2}$, and $\alpha \Re \beta$, then $\alpha$ and $\beta$ agree in their specifications for the feature Feat

(I.e., "corresponding segments in the Corr relation have identical specifications for Feat." On IDENT constraints and correspondence theory, see McCarthy \& Prince 1995.)

Thus, any individual IDENT constraint that is built from this schema has a formulation that is compositional, given the formulation of the schema and the specific choice of arguments for the Corr and Feat variables.

Another example of a constraint schema is the general schema for positional constraints, both markedness (M/str) and faithfulness (F/str), ${ }^{4}$ as in (6). (This schema is discussed in more detail in $\S 2.2 .2$ below.)

The $\mathbf{C} /$ str schema

$\mathrm{C} /$ str

For all $y$, if $y$ is a str, then $\mathbf{C}$ holds of $y$

where $y$ is an element in the focus of the constraint $\mathbf{C}$ (see $§ 2.2 .2$ for elaboration)

The schemas and the constraints that they build must be divided into the categories $\mathbf{M}$ and $\mathbf{F}$, so that filters can make reference to either markedness or faithfulness constraints-for example, the Prominence Condition is a filter that specifically applies to $\mathbf{M} / \mathbf{s t r}$ constraints (and not to, say, F/str constraints). However, the labels $\mathbf{M}$ and $\mathbf{F}$ need not be independently stipulated, because they can be derived from the content of the schema formulations themselves. Any

3. The set of possible correspondence relations over which faithfulness constraints can be defined includes the I(nput)-O(utput), O(utput)-O(utput), and $\mathrm{B}$ (ase)-R(eduplicant) relations (McCarthy \& Prince 1995; Benua 1995, 1997; Burzio 1994, 1997).

4. The use of a general $\mathrm{C} /$ str schema here, rather than a more specific $\mathbf{M} /$ str schema, assumes that the constraints responsible for positional neutralization effects (that is, contrast preservation specifically in strong positions) are F/str constraints; see $\S 5.2$ for a discussion of alternatives. 
schema or constraint that makes reference to a correspondence relation is $\mathbf{F}$, and all others are $\mathbf{M}$.

In addition to a set of constraint schemas, the constraint-construction module also contains a set of primitive phonological elements, which serve as the arguments for the variables in the schemas. These include, for example, the set of phonological features Feat, the set of edges Edge, the set of correspondence relations Corr, and the set of prosodic constituents $P C a t$. Another set of primitive elements in the Schema/Filter model is the set of strong positions str to which constraints can be relativized. The set of strong positions is further subdivided into the set of phonetically strong positions $\Phi_{s}$ tr and the set of psycholinguistically strong positions $\Psi_{s t r}$; this division is necessary because some filters, including the Segmental Contrast Condition ( $(2.4)$, are sensitive to the difference between $\Phi_{s t r}$ and $\Psi_{\text {str }}$ (see also $§ 2.4 .3$ for another example of a filter that is sensitive to this difference).

It should be emphasized that the strong positions included in the sets $\Phi_{S t r}$ and $\Psi_{\text {str }}$ are formal phonological objects-they derive their special status from their characteristic phonetic or psycholinguistic salience, but they are not supplied directly by the phonetic or the psycholinguistic component of the grammar. This must be the case, as two examples will illustrate. First, phonetically strong positions typically have special status because they possess salient cues to the recovery of particular contrasts (\$2.4.3). However, a positional augmentation (M/str) constraint can make reference to a phonetically strong position even when the force of the general $\mathbf{M}$ constraint from which $\mathbf{M} / \mathbf{s t r}$ is built has no relationship to the featural contrast for which the position has special salient cues. E.g., the special featurelicensing abilities of the phonetically strong position stressed syllable are apparently limited to vowel features and suprasegmentals like tone ( $\$ 2.4 .3)$, but augmentation constraints like ONSET and the *ONSET/X subhierarchy, which manipulate consonantal features, can nevertheless be relativized to the position stressed syllable ( $\S 3.2)$. Thus, the status of the stressed syllable as a strong position is more abstract and general than the phonetic origin of that privileged status.

A second example of why the strong positions must be treated as abstract formal objects is seen in the designation of the initial syllable as a psycholinguistically strong position. This position has special status because material toward the beginning of the word has a particularly large influence on early-stage word recognition (see $\S 4.3 .2$ for detailed discussion). But while the psycholinguistic importance of material in a word seems to fall off gradually from left to right, the phonological reflection of this importance is categorical; the initial syllable is a privileged position, but there is no sense in which, for example, the third syllable has special phonological status compared to the fourth. 
An open question at this point is the ultimate source of the basic elements of the system: the schemas and their arguments. Some of them may be innate, part of UG. However, at least some of these fundamental phonological elements may be learned as part of the process of child language acquisition. See, for example, Hayes (1999ab) and Boersma (1998) for proposals concerning ways in which aspects of the constraint set might be learnable. ${ }^{5}$

To summarize, the constraint-construction module of the Schema/Filter model of CON has the following properties. Any constraint schema can apply to any argument, as long as it is of the appropriate type (i.e., a feature must replace a Feat variable, and cannot replace Edge or Corr or PCat). Also, any constraint, whether $\mathbf{M}$ or $\mathbf{F}$, can be relativized to any member of the set of strong positions. ${ }^{6}$ Crucially, other than argument/variable type-matching, there are no restrictions at this stage. Thus, all formally possible constraints are constructed by the constraint metagrammar, including constraints that are not empirically attested-but this "overgeneration" of constraints is corrected by the filter module, discussed in the following subsection.

Thoroughly defending the claim that every constraint in the universal inventory is constructed from some schema is beyond the scope of this dissertation. It may be the case that a few constraints that are not decomposable into schemas and arguments are also supplied by UG and appear in CON. However, any constraint that is a member of a family of constraints, including those mentioned above and a number of others as well ( ${ }^{*}$ Feat; MAX-Corr-PCat; . . ) ), is best viewed as the output of a schema. Most important for now is the fact that the Schema/Filter model

5. A related question is this: Are the constraints literally constructed from the schemas as part of the language acquisition process (Hayes 1999a, Boersma 1998)? Or does the notion of "constraint construction" apply at a more abstract level, so that the analysis of the constraint set into schemas, arguments, and filters is simply a descriptive model or, perhaps, an evolutionary model? The former hypothesis is more attractive, in that it reduces the amount of phonological knowledge that would have to be innate, while still allowing for CON to be universal. At the very least, as noted above, alignment constraints that refer to specific morphemes or morpheme classes in a language must be actively acquired by means of the generalizable ALIGN schema, because information about individual morphemes cannot be innate.

6. There is a general condition on relativized constraints that rules out any $\mathbf{C} / s t r$ constraint in which the domain of application of $\mathbf{C}$ is larger than, or otherwise incompatible with, the size of the strong position in question. For example, the $\mathbf{M} / \mathbf{s t r}$ constraint HAVECPLACE/V: is ruled out because HAVECPLACE is evaluated within the domain of a consonant (see \$2.3.2.4 below), and there is no consonant contained within the strong position V: (long vowel). See $\S 2.2 .2$ and $\$ 2.3 .3$ for further discussion of such cases of domain mismatch. 
provides an explicit framework within which the nature of the constraints in CON can be investigated. Furthermore, this system has a number of desirable characteristics. It reduces to a set of universal primitives (the schemas and their arguments). Moreover, as with ALIGN(Root, L, PrWd, L) in (4) above, constraint formulations become transparently compositional, given the formulation of the schema from which each constraint is built.

\subsubsection{Constraint filters}

The preceding subsection has shown that it is advantageous to develop a model of CON in which constraints are constructed from a set of basic elements. However, any such theory must also be able to handle the problem of formally possible constraints that nevertheless do not exist. The Schema/Filter model of CON addresses this problem with a set of constraint filters. Constraint filters make use of substantive information to block constraints that are formally possible (and thus are emitted by the constraint-construction module), but are not appropriate constraints on substantive grounds.

As the discussion in $\S 1.2$ has shown, the difference between formally possible $\mathbf{M} / \mathbf{s t r}$ constraints that exist and those that do not is a matter of whether or not the $\mathbf{M} / \mathbf{s t r}$ constraint in question satisfies certain substantive requirements: any $\mathbf{M} / \mathbf{s t r}$ constraint must be an augmentation (prominence-enhancing) constraint, and if it is an $\mathbf{M} / \Psi$ str constraint, its satisfaction must not entail the loss of a crucial phonological contrast.

More generally, there are many cases in which a formally possible constraint - one that has the same formal structure as a constraint that is empirically attested - does not exist, but its nonexistence is understandable on substantive grounds. For example, given the FEATCO-OCCUR schema and the set of features shown in (7), both the attested constraints in (8a) and the unattested constraints in (8b) can be constructed.

(a) FEATCO-OCCUR schema: ${ }^{*}\left[\right.$ Feat $_{1}$, Feat $\left._{2}\right]$

(b) Feat $\in\{ \pm$ son, \pm hi, \pm voi, lab, RTR, ATR,$\ldots\}$ 
Formally possible FEATCO-OCCUR constraints

(a) Attested FEATCO-OCCUR constraints

*[-son, +voi] (Westbury \& Keating 1986; Stevens \& Keyser 1989)

*[+hi, RTR] (Archangeli \& Pulleyblank 1994)

(b) Unattested FEATCO-OCCUR constraints

$$
\begin{aligned}
& *[+ \text { son, lab }] \\
& *[+ \text { hi, ATR }]
\end{aligned}
$$

Thus, an adequate model of CON must be able to exclude the constraints in (8b). ${ }^{7}$

The difference between the actual constraints in (8a) and the formally possible, but nonexistent, constraints in $(8 \mathrm{~b})$ is that the former are functionally grounded in the sense of Archangeli \& Pulleyblank (1994)-there are articulatory and/or perceptual reasons why constraints such as these should exist. For example, the existence of the constraint *[-son, +voi] reflects the fact that voiced obstruents are articulatorily more difficult (Westbury \& Keating 1986), and perceptually less distinct (Stevens \& Keyser 1989), than voiceless obstruents or voiced sonorants. Similarly, since the feature [+high] involves an upward and forward movement of the tongue body, which lowers F1, while the feature [RTR] involves a downward and backward movement of the tongue root, which raises $\mathrm{F} 1$, the constraint $*[+$ hi, RTR] also reflects articulatory difficulty and conflicting auditory cues. But feature pairs that are articulatorily and perceptually unrelated, like [+sonorant] and [labial], or mutually enhancing, like [+high] and [ATR], do not combine to form legitimate feature co-occurrence constraints.

The principle of Inductive Grounding proposed by Hayes (1999a) provides a way to distinguish between the constraints in (8a) and those in (8b). Inductive Grounding ensures that a constraint of the FEATCO-OCCUR type (as well as similar kinds of constraints, such as constraints on the featural context in which a particular segment can occur) is part of CON only if segments or sequences that violate the

7. This problem must be addressed in any model of CON. The current proposal makes it easy to see that a constraint like *[+hi, ATR] is predicted to exist and must somehow be ruled out, because the model explicitly includes a general FEATCO-OCCUR constraintbuilding schema. However, the question of why there is a constraint $*[+h i, R T R]$ and not a constraint *[+hi, ATR] is independent of the choice of a model that postulates constraint schemas. 
constraint are more difficult, articulatorily (or perceptually), than segments or sequences that satisfy it. ${ }^{8}$ The unattested constraints in (8b) fail this filter; for * [ +son, lab], the segments that violate the constraint are no more phonetically difficult than those that satisfy it, and for *[+hi, ATR], the results are completely wrong, since segments that violate this constraint are phonetically better than (a subset of) those that satisfy it. On the other hand, the FEATCO-OCCUR constraints in (8a) pass Inductive Grounding, so they are included in CON.

Hayes (1999a) observes that there is an important consequence of the use of the principle of Inductive Grounding to determine which FEATCO-OCCUR constraints are part of CON and which are not. Namely, this approach provides a way for functional grounding to have a major impact on the constraint system, rather than merely being some kind of extragrammatical description of the system-while still allowing for a formal theory of phonology, which accounts for the fact that many phonological phenomena are categorical, reflect phonological constraints that are logically simple in structure, and involve classes or sets of elements defined over somewhat abstract, if often phonetically based, properties.

The Schema/Filter model of CON extends Hayes' (1999a) approach, resulting in a general model of how substantive grounding can shape the phonological system. In this model, the output of the constraint-construction module, which consists of all formally possible constraints, passes through a set of constraint filters (see (2) above). The filters determine which of the formally possible constraints are in fact included in CON. The set of constraint filters includes the Inductive Grounding Principle of Hayes (1999a) and also filters instantiating all other substantively based restrictions that hold of the constraints in CON. Thus, two of the constraint filters in the system are the Prominence Condition on M/str constraints (§2.3) and the Segmental Contrast Condition on $\mathbf{M} / \Psi$ str constraints (§2.4). (Another possible filter, that restricts positional faithfulness constraints for phonetically strong positions ( $\mathbf{F} / \boldsymbol{\Phi}$ str), is considered in $\$ 2.4 .3$ below.)

In this model, it is a characteristic of all constraint filters that, like the Inductive Grounding Principle, the Prominence Condition, and the Segmental Contrast Condition, they make use of information from outside the formal phonological system to determine which of the formally possible constraints are actually included in CON. Thus, the constraint filters are the point of intersection between the formal phonological system and substantive or functional considerations. The articulatory

8. See Hayes (1999a) for further discussion of the principle of Inductive Grounding, which also takes into account the formal simplicity of the constraints being evaluated: a constraint passes Inductive Grounding if it makes a better partition of the phonetic space into "easy" and "difficult" than any constraint of equal or greater formal simplicity. 
phonetics, the perceptual system, the language processor, and other such sources of functional influence do not create constraints. However, through the constraint filters, these systems have what amounts to veto power over the formal constraints that are created. As a result, the structure of CON reflects substantive and functional concerns even though it contains only objects created by a formal system. ${ }^{9}$

In the case of $\mathbf{M} / \mathbf{s t r}$ constraints, the $\mathbf{C} /$ str constraint schema allows any markedness constraint to be relativized to any strong position, because all that a schema does is to apply its function to any and all relevant arguments (here, to constraints-including markedness constraints-and strong positions). Functional grounding enters the picture in the form of the constraint filters, in this case, the Prominence Condition and the Segmental Contrast Condition. (The kinds of substantive information that are relevant for these filters, and the way in which constraints are tested for compliance with the filters, are discussed in $\$ 2.3$ and §2.4.)

The Schema/Filter model thus operates as in (9).

The Schema/Filter model of CoN, with example constraints

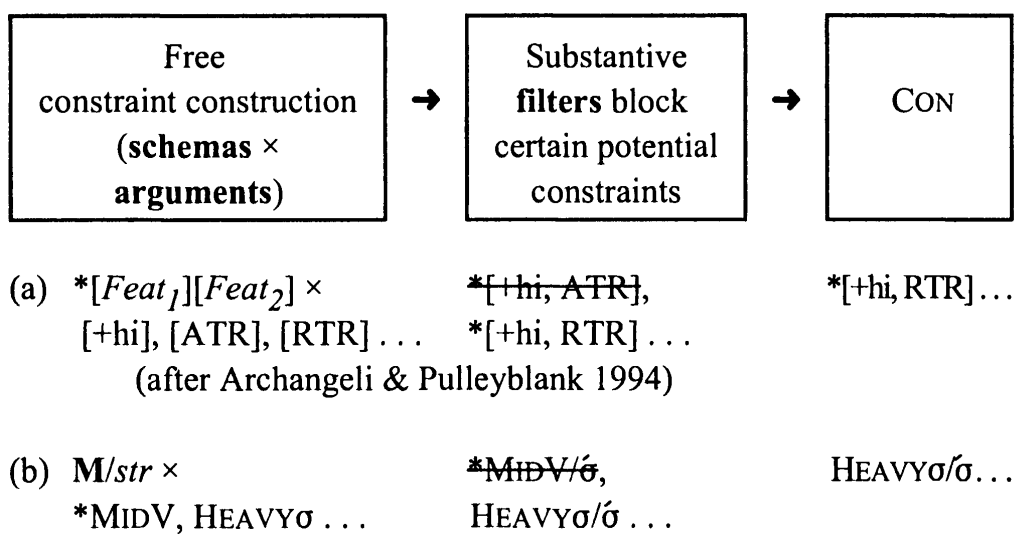

Constraint schemas are free to apply to all arguments of the correct type. If the output of a particular schema has no associated filters, then all formally possible constraints built from that schema will be included in CON, and there will be no

9. For other approaches to the functional grounding of constraints and/or phonological processes, see, e.g., Steriade (1993, 1997, 1999ab), Archangeli \& Pulleyblank (1994), Flemming (1995), Ní Chiosáin \& Padgett (1997), Boersma (1998), and Pater (1999). For another use of the perceptual system to evaluate phonological alternatives, see the targeted constraints of Wilson $(2000,2001)$. 
distinction between constraints of that type that are somehow substantively grounded and constraints of that type that are not. ${ }^{10}$ However, for many constraint types, such as FEATCo-OCCUR constraints or $\mathbf{M} / \mathbf{s t r}$ constraints, there are substantive filters that pass only a subset of the possible constraints. Thus, the universal constraint set CON is shaped by formal considerations, because all constraints are built from a basic phonological inventory of schemas and arguments, and substantive considerations, because for certain constraint types there are filters encoding extraphonological, substantive information that determine which of the formally possible constraints are ultimately part of CON.

The question of how $\mathbf{M} / \mathbf{s t r}$ constraints, in particular, are affected by constraint filters is taken up in $\$ 2.3$ and $\$ 2.4$ below. First, however, there is one more general theoretical point about constraints to be addressed: how the formulation of a relativized or position-specific constraint (such as an $\mathbf{M} / \mathbf{s t r}$ constraint) is to be derived, compositionally, from the formulation of the general version of the constraint. This question, which has crucial implications for determining which $\mathbf{M} / \mathbf{s t r}$ constraints can legitimately be relativized to which positions, is the topic of the following section.

\subsubsection{A compositional approach to relativized constraints}

One of the advantages of a system in which constraints are built from general constraint schemas is that the formulation of each specific constraint becomes predictable from the formulation of the general schema used to construct the constraint in question, plus the semantic contributions of the specific arguments that fill the variables in the schema. For example, the formulation of the individual alignment constraint in (10b) is transparently related to the formulation of the general ALIGN schema in (10a) plus the arguments Cat $1=$ Root, Cat $2=\operatorname{PrWd}$, $E d g e 1=\mathrm{L}$, and $E d g e 2=\mathrm{L}$ (schema and constraint repeated here from (3), (4) above).

10. This may be the case for (non-positional) faithfulness constraints, since $\mathbf{F}$ constraints demand the preservation of input characteristics (rather than structural well-formedness). 
(10) The compositional formulation of alignment constraints

(a) The ALIGN schema (adapted from McCarthy \& Prince 1993a:80)

$\operatorname{Align}($ Cat 1, Edge1, Cat2, Edge2)

$\forall$ Cat $1 \exists$ Cat 2 such that Edge 1 of Cat 1 and Edge2 of Cat 2 coincide

where Cat 1, Cat $2 \in$ PCat $\cup$ MCat

Edge1, Edge $2 \in\{\mathrm{R}(\mathrm{ight}), \mathrm{L}(\mathrm{eft})\}$

(b) An individual example of an alignment constraint
ALIGN(Root, L, PrWd, L)
$\forall$ Root $\exists \operatorname{PrWd}$ such that Edge $=\mathrm{L}$ of Root and Edge $=\mathrm{L}$ of PrWd coincide

Descriptively, $\mathbf{M} / \mathbf{s t r}$ constraints are formed by relativizing a markedness constraint to a strong position. Under the Schema/Filter model of CON, this means that there is a constraint schema, the $\mathbf{C} /$ str schema, that takes a constraint and relativizes it to one of the elements in str, the set of strong positions. This constraint schema is defined as in (11).

The C/str schema

C/str For all $y$, if $y$ is a str, then $\mathbf{C}$ holds of $y$

where $y$ is an element in the focus of the constraint $\mathbf{C}$

The $\mathbf{C} /$ str schema functions as a general schema that can relativize any constraint to any strong position, while still compositionally generating a meaningful formulation for the relativized constraint, because it makes crucial reference to the focus of the constraint that it takes as one of its arguments. The concept of a constraint focus is developed by Crowhurst \& Hewitt (1997), who describe it as follows and formalize it as in (12).

... every constraint has a FOCUS, which may be defined as the linguistic object upon which some condition of maximum harmony is predicated. Abstracting away from differences due to style, we recognize at the heart of any constraint a 
definition of a state of maximum harmony holding on some linguistic object in relation to some other linguistic object. (Crowhurst \& Hewitt 1997:9)

(12) The focus of a constraint (Crowhurst \& Hewitt 1997:10)

i. Every constraint has a unique focus.

ii. A constraint's focus is identified by the universally quantified argument.

Thus, for every constraint, a constraint focus can be identified. The $\mathbf{C} /$ str schema takes advantage of this universal fact about constraint formulations. This schema embeds the formulation of any general constraint $\mathbf{C}$ inside an if-then statement, such that if a particular element from the focus of $\mathbf{C}$ is an instance of a chosen strong position, then $\mathbf{C}$ must hold of that element - but if the element under scrutiny is not an instance of the strong position in question, then, as desired, the positional constraint is vacuously satisfied whether $\mathbf{C}$ actually holds of that element or not.

For example, consider the M/str constraint ONSET/ó (§3.2.2), a positional version of ONSET that is relativized to the strong position stressed syllable. The general constraint ONSET has the formulation in (13) (see \$2.3.2.3.2 for discussion), requiring the head (peak) of a syllable to be preceded by something else in the syllable.

ONSET For all syllables $x, a \neq b$

where $a$ is the leftmost segment dominated by $x$

$b$ is the head of $x$

The focus of any constraint is the element associated with universal quantification, so in the case of ONSET, the focus is the syllable.

When ONSET becomes an argument of the $\mathbf{C} /$ str schema, repeated in (14), along with a designated strong position, such as ó, the positional constraint in (15) is the result.

C/str For all $y$, if $y$ is a str, then $\mathbf{C}$ holds of $y$

where $y$ is an element in the focus of the constraint $\mathbf{C}$ 
ONSET/ó For all syllables $x$, if $x$ is a ó, then $a \neq b$

where $a$ is the leftmost segment dominated by $x$ $b$ is the head of $x$

In some cases, a positional constraint built from the $\mathrm{C} /$ str schema will be anomalous, because the strong position to which the constraint is relativized is not the same kind of unit as that found in the focus of the constraint. One such example is ONSET/V: (16), a version of ONSET relativized to the strong position long vowel.

\section{ONSET/V: For all syllables $x$, if $x$ is a V:, then $a \neq b$}

where $a$ is the leftmost segment dominated by $x$ $b$ is the head of $x$

This positional constraint is well-formed with respect to the constraint schema $\mathrm{C} /$ str, which simply combines constraints and strong positions. However, it is a meaningless constraint, because a syllable and a long vowel are distinct classes of phonological objects. Informally, it is meaningless to require that a long vowel have an onset, since it is syllables, not vowels themselves, that have onsets. Formally, the problem is that the antecedent clause in (16), 'if [syllable] $x$ is a V:,' will always be false; a conditional with an antecedent that is false is itself always true, so a constraint such as ONSET/V: will be (vacuously) satisfied by every output candidate. By definition, a constraint like this will never be 'active' on a candidate set-that is, it will never demarcate a proper subset of the candidate set as suboptimal (Prince \& Smolensky 1993).

The anomaly that arises when the focus of a constraint is a different class of object from the strong position to which the constraint has been relativized will be called a domain mismatch. For the sake of explicitness, relativized constraints involving a domain mismatch are assumed not to be included in CoN; i.e., there is assumed to be a constraint filter that screens them out. However, nothing crucial hinges on this assumption, because even if such constraints were included in CON, they would simply have no effect on the selection of optimal output forms in any language.

In $\$ 2.3 .2$ below, a number of markedness constraints that have $\mathbf{M} / \mathbf{s t r}$ counterparts are discussed. Explicit formulations are given for each markedness constraint. As outlined here, it is the nature of the focus of each constraint that determines the strong positions to which it can be meaningfully relativized, as opposed to those positions that lead to a domain mismatch. 


\subsubsection{Summary}

This section has laid out several aspects of a general theory of constraints within which the subsequent discussion of $\mathbf{M} / \mathbf{s t r}$ constraints can be carried out. First, the question of how substantive considerations affect the universal constraint set was addressed. In the Schema/Filter model of CoN, constraints are built from basic phonological elements (such as features and prosodic constituents) according to a set of constraint schemas. All formally possible combinations of schemas and the basic elements that serve as their arguments can be constructed. However, there are substantive filters that operate on the output of constraint construction, allowing only some of the formally possible constraints to be included in CON. The advantage of the Schema/Filter model is that it provides a way for phonology (that is, CON, and therefore also individual language-particular constraint rankings) to be influenced and shaped by functional or substantive considerations, while remaining a formal system that manipulates formal objects in a constrained way.

In the Schema/Filter model, it is constraint schemas that determine, compositionally, the formulations of the individual constraints that they construct. In other words, every constraint built from a particular schema has a formulation that is predictable from the formulation of the schema plus the phonological elements involved in the specific constraint. Therefore, a general theory of $\mathbf{M} / \mathbf{s t r}$ constraints must provide a way for the formulation of every $\mathbf{M} / \mathbf{s t r}$ constraint to be compositionally determined from the formulation of the general markedness constraint and the particular strong position to which the constraint has been relativized. This is accomplished by defining the $\mathrm{C} /$ str schema-the schema responsible for relativized constraints - so that it embeds the original formulation of a general constraint $\mathbf{C}$ into an if/then clause that is sensitive to the focus of the general constraint: if the focus of $\mathbf{C}$ is an instance of str, then the property demanded by $\mathbf{C}$ must hold.

\subsection{M/str constraints and the Prominence Condition}

Now that a general model of constraints, constraint formulations, and the structure of CON has been laid out, a theory of positional augmentation constraints can be developed within this framework. The first component of such a theory is the Prominence Condition - the restriction of $\mathbf{M} / \mathbf{s t r}$ constraints to those that enhance the perceptual prominence of the strong position to which they are relativized. In $\S 2.3 .1$, the Prominence Condition is formally modeled as one of the filters in the Schema/Filter model. Then, $\S 2.3 .2$ demonstrates that the predictions made by the 
Prominence Condition as a filter on $\mathbf{M} / \mathbf{s t r}$ constraints are correct: the individual markedness constraints that are observed to have $\mathbf{M} / \mathbf{s t r}$ counterparts are all shown to be augmentation (prominence-enhancing) constraints. Finally, $\$ 2.3 .3$ considers what happens when the general augmentation constraints discussed in $\$ 2.3 .2$ are relativized to the various strong positions, showing which positional augmentation constraints are meaningful constraints and which give rise to a domain mismatch.

\subsubsection{The Prominence Condition and enhancement of perceptual prominence}

In many languages, such as those discussed in chapters 3 and 4 , there are phonological requirements enforced specifically of strong positions. For example, in Mohawk (\$3.2.1.1), stressed syllables, but not other syllables, are required to be heavy. In Chamicuro (\$3.4), onsets, but not other consonants, are required to have supralaryngeal Place specifications. In Arapaho (\$4.2.1.1), initial syllables, but not other syllables, are required to have onsets. Such effects are observed in a language when a high rank is assigned to $\mathbf{M} / \mathbf{s t r}$ constraints, markedness constraints that specifically refer to strong positions $(\S 1.3 .2)$. However, as demonstrated in $\S 1.2$, there are formally possible $\mathbf{M} / \mathbf{s t r}$ constraints that do not exist, such as *MIDV/ó, a constraint that would ban mid vowels in stressed syllables. Therefore, a theory of $\mathbf{M} / \mathbf{s t r}$ constraints must predict which of the formally possible $\mathbf{M} / \mathbf{s t r}$ constraints are actually attested.

The proposal developed here is that $\mathbf{M} / \mathbf{s t r}$ constraints are restricted by the Prominence Condition, an informal statement of which is repeated in (17).

\section{The Prominence Condition}

Markedness constraints specific to strong positions are included in CON only if the general markedness constraints from which they are built call for the presence of perceptually prominent properties.

The Prominence Condition thus states that the only legitimate $\mathbf{M} / \mathbf{s t r}$ constraints are those that are positional versions of augmentation (prominence-enhancing ${ }^{11}$ )

11. The term enhancement as used here, in the context of enhancement of the perceptual prominence of a strong position, is not related to featural enhancement in the sense of Stevens \& Keyser (1989). Featural (or contrast) enhancement refers to the tendency that languages have to assign values for non-contrastive features such that they enhance the 
constraints. This requirement correctly predicts the existence of the $\mathbf{M} / \mathbf{s t r}$ constraints responsible for the empirically attested requirements on strong positions listed above, and the non-existence of empirically problematic $\mathbf{M} / \mathbf{s t r}$ constraints such as the putative *MIDV/ó.

The concept behind the Prominence Condition is this. The M/str constraints that comply with the Prominence Condition are exactly those constraints that take a strong position, which by definition is already prominent along some phonetic or psycholinguistic dimension, and require it to become even more prominent through association with some perceptually salient property. When $\mathbf{M} / \mathbf{s t r}$ constraints are satisfied, "the strong get stronger."

This characteristic is consistent with a general pattern that many markedness constraints follow: a mandate for the co-occurrence of mutually reinforcing properties. For example, Stevens \& Keyser (1989) argue that certain feature cooccurrence patterns are marked because they give rise to conflicting cues in the acoustic signal-e.g., prototypical obstruents have no low-frequency energy, because sonorants do; voicing an obstruent adds low-frequency energy to the signal; so voiced obstruents are marked. Restating Stevens \& Keyser's (1989) claim in OT terms, there is a markedness constraint requiring obstruents to be voiceless, because voicelessness makes obstruents more like prototypical obstruents. The cooccurrence of mutually reinforcing properties can also be seen as the basis of the operation of "harmonic alignment" (Prince \& Smolensky 1993:67, 136), which forms universal constraint subhierarchies like *ONSET/X and *PEAK/X that favor an association between the prominent ends of two scales (e.g., syllable peaks and high-sonority segments) and the non-prominent ends of those scales (e.g., syllable onsets and low-sonority segments).

Conversely, M/str constraints that are not augmentation constraints, such as the putative *MIDV/ó, would if anything make strong positions less prominent by stripping away potential phonological contrasts without adding to the perceptual salience of the position. With the Prominence Condition in place, such constraints are correctly predicted not to be included in CON.

perceptual difference between the opposing values for a feature that is contrastive. For example, if [ \pm round] is non-contrastive for vowels in a language, then usually front vowels will be redundantly [-round] and back vowels will be redundantly [+round]. This patterning of the non-contrastive feature [tround] is said to "enhance" the contrast between front and back vowels, because it increases the difference in their F2 values that is the primary acoustic correlate of the front/back contrast. On the other hand, enhancement of perceptual prominence refers to an increase in the perceptual salience of a given entity (segment, syllable, morpheme), as measured by, e.g., magnitude of neural response to that entity as a stimulus. 
In the Schema/Filter model of CoN, the Prominence Condition can be formally implemented as a member of the set of constraint filters. Thus, any markedness (or faithfulness) constraint can be relativized to any strong position by means of the $\mathbf{C} / s t r$ constraint schema. However, any $\mathbf{M} / \mathbf{s t r}$ constraint thus constructed must be tested by the Prominence Condition, now explicitly modeled as a substantively based constraint filter that is designated to apply to $\mathbf{M} / \mathbf{s t r}$ constraints (18).

\section{The Prominence Condition (as a constraint filter)}

If a constraint is of the form $\mathbf{M} / \mathbf{s t r}$, then it must meet the following condition:

M must be an augmentation constraint, i.e., a constraint that calls for the presence of a perceptually prominent property. ${ }^{12}$

A formally possible $\mathbf{M} / \mathbf{s t r}$ constraint that does not meet the requirements imposed by the Prominence Condition, such as *MidV/ó, is not passed by this filter and so is not included in the set of universal constraints from which language-particular OT grammars are formed.

As is the case with all constraint filters in the Schema/Filter model (see $\S 2.2 .1 .2$ ), the Prominence Condition makes use of substantive information from outside the formal phonological system when it evaluates constraints for compliance. In this case, the crucial information has to do with perceptual prominence: are candidates that satisfy a given markedness constraint more perceptually prominent than candidates that violate the constraint? If so, the constraint passes the Prominence Condition.

Perceptual prominence itself can perhaps be measured in terms of neural response, since auditory-nerve firing rate is known to be higher when signal intensity is greater (Delgutte 1997, Geisler 1998). That is, it may be appropriate to categorize one stimulus as more perceptually prominent than another if the first stimulus elicits a neural response of greater magnitude than that elicited by the second. However, the task of the Prominence Condition-to decide whether a particular M/str constraint acts to enhance perceptual prominence-is somewhat more complex than comparing the neural responses produced by a given pair of

12. By hypothesis, the Prominence Condition tests the general version of the constraint, rather than the relativized version, for its ability to enhance perceptual prominence. The status of a constraint as an augmentation constraint is therefore independent of any strong position it might be relativized to. 
stimuli. It is also necessary to ensure that the stimuli to be compared are the appropriate ones.

In the case of the Inductive Grounding Principle, Hayes (1999a) proposes that a feature-co-occurrence or feature-context constraint (such as *[-son, +voi]) is inductively grounded if the boundary between feature combinations that satisfy the constraint and those that violate it correctly partitions speakers' general-knowledge "map of phonetic difficulty" into regions of easier and more difficult articulations (§2.2.1.2). But in the case of the Prominence Condition, which must determine whether a particular markedness constraint is one that calls for the presence of perceptually prominent characteristics, it is not enough just to see whether all (or most) candidates that satisfy the constraint are more perceptually prominent than all (or most) candidates that violate it. For example, the markedness constraint *[labial] is violated by [pe] and satisfied by [ke:ta]. The latter candidate, with a long vowel and an additional syllable, will certainly give rise to a larger neural response than the former, simply because it has a longer overall duration. However, intuitively, this fact should not be used to show that *[labial] is an augmentation constraint, because factors other than the difference between $[\mathrm{p}]$ and $[\mathrm{k}]$ are what really make the second candidate more perceptually prominent than the first.

Therefore, to test whether a particular markedness constraint $\mathbf{M}$ qualifies as an augmentation constraint (and so may have an $\mathbf{M} / \mathbf{s t r}$ counterpart), it is necessary to compare a pair of output candidates that are as close to identical as possible in all respects other than whatever property determines the satisfaction or violation of $\mathbf{M}$. That is, the candidates that are compared must be a kind of "minimal pair." The compliance-testing procedure for the Prominence Condition will thus be something like the following.

For a given markedness constraint $\mathbf{M}$, choose an arbitrary input and consider a set of two minimally different output candidates such that one satisfies $\mathbf{M}$ and one violates M. Minimally different output candidates are those whose input-output faithfulness violations, computed with respect to the arbitrary input, differ only in that one candidate has one fewer violation of one faithfulness constraint than the other candidate has. (In cases where the violation of one faithfulness constraint entails the violation of others, minimally different candidates are those that differ by the smallest number of faithfulness violations possible). Because the two candidates differ only in one aspect of their faithfulness to the arbitrary input, they are nearly identical. This requirement forces their one point of difference to be relevant to the demands of the $\mathbf{M}$ being tested, since the two output candidates must differ in their satisfaction of $\mathbf{M}$, but can only differ in one property. Under these conditions, if the M-satisfying candidate is judged to be more prominent than the $\mathbf{M}$-violating candidate when the two are fed to the perceptual system, then $\mathbf{M}$ is an 
augmentation constraint, and any $\mathbf{M} / \mathbf{s t r}$ constraint constructed from $\mathbf{M}$ passes the Prominence Condition.

For example, consider the M/str constraint HEAVYo/o. The M constraint from which it is built is HEAVYo ( $\$ 2.3 .2 .1)$, so an arbitrary input and two minimally different output candidates as specified above are considered with respect to this constraint. The faithfulness constraint by which these candidates differ is MAX- $\mu$, which penalizes the deletion of an input mora (McCarthy \& Prince 1995; Itô, Kitagawa, \& Mester 1996; McCarthy 2000).

Testing Heavyo/str for compliance with the Prominence Condition

\begin{tabular}{|c||c|}
\hline /ta:/ & HEAVYo \\
\hline \hline a. ta: & \\
\hline b. ta & $*$ \\
\hline
\end{tabular}

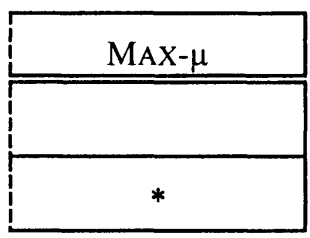

Since candidate (19a), which satisfies HEAVYo, is more perceptually prominent (see $\S 2.3 .2 .1)$ than candidate (19b), which violates the constraint, this means that HEAVYo qualifies as an augmentation constraint, so the M/str constraint HEAVYo/ó is well-formed according to the Prominence Condition. Note that the same result would emerge if the arbitrary input had been /ta/, with a short vowel, since (19a) would still be more perceptually prominent than (19b). ${ }^{13}$ It is not relevant which of the two output candidates is more faithful to the arbitrary input; they must just be minimally different in their faithfulness violations.

This proposal for how the Prominence Condition uses information from the perceptual system to identify augmentation (prominence-enhancing) constraints is somewhat preliminary and may need to be further refined as additional cases are considered. For example, as noted above, the putative stressed-syllable markedness constraint *MIDV/ó is not a member of CoN, and in fact including this constraint in CoN would allow for a pattern that does not occur: the avoidance of specifically mid vowels in stressed syllables. The absence of *MIDV/ó from CON is correctly predicted by the general principle behind the Prominence Condition, since forcing vowels to be peripheral rather than mid is not fundamentally a means of enhancing perceptual prominence (as discussed in $§ 2.3 .2 .3$, some non-mid vowels, namely

13. In that case, the faithfulness constraint on whose satisfaction the two candidates differ would be DEP- $\mu$ (penalizing the insertion of a mora) rather than MAX- $\mu$, but there would still be only a single difference in faithfulness violations between the two candidates. 
high vowels, are less perceptually salient than mid vowels are). However, care must be taken that the specific implementation given to the Prominence Condition as part of the Schema/Filter model does not allow *MIDV/str to slip into CoN. The problem is that mid vowels are in fact less perceptually prominent than some peripheral vowels, i.e., low vowels $(\$ 2.3 .2 .3)$. It is therefore possible to imagine a scenario where *MIDV is tested for compliance with the Prominence Condition and the arbitrary input happens to contain a low vowel.

(20) Testing * MIDV for compliance with the Prominence Condition

\begin{tabular}{|c|c|c|c|}
\hline$/ \mathrm{tæ} /$ & $* \mathrm{MIDV}$ & $\ldots$ & IDENT[Vht] \\
\hline \hline a. tæ & & & \\
\hline b. te & $*$ & & $*$ \\
\hline
\end{tabular}

In this case, the candidate that satisfies *MIDV, (20a), does happen to be more perceptually prominent than the candidate that violates the constraint, (20b). Yet this is only a coincidence. If the arbitrary input had been /ti/, the reverse result would have been true: the candidate satisfying *MIDV ([ti]) would have been less perceptually prominent than its competitor ([te]). Therefore, it may be necessary for the Prominence Condition to test multiple arbitrary inputs when it examines a given markedness constraint in order for it to determine whether the property mandated by that constraint is truly one that enhances perceptual prominence--as opposed to one, like * MIDV, whose satisfaction may under some circumstances coincidentally increase perceptual salience.

The following subsection ( $\$ 2.3 .2)$ examines the predictions made by implementing the Prominence Condition as a member of the set of substantively based constraint filters, and shows that these predictions match empirical patterns; each markedness constraint known to have an $\mathbf{M} / \mathbf{s t r}$ counterpart is shown to be a constraint that does indeed call for the presence of a perceptually prominent property.

\subsubsection{Augmentation constraints}

The $\mathbf{M} / \mathbf{s t r}$ constraints exemplified in chapters 3 and 4 are positional versions of the following set of constraints. 
(21) Markedness constraints with $\mathbf{M} / \mathbf{s t r}$ counterparts

$\begin{array}{ll}\text { HEAVYo } & (\S 2.3 .2 .1) \\ { }^{*} \text { PEAK/X } & (\S 2.3 .2 .2) \\ \text { ONSET } & (\S 2.3 .2 .3 .2) \\ { }^{*} \text { ONSET/X } & (\S 2.3 .2 .3 .3) \\ \text { HAVECPLACE } & (\S 2.3 .2 .4) \\ \text { HTONE } & (\S 2.3 .2 .5) \\ \text { HAVESTRESS } & (\S 2.3 .2 .6)\end{array}$

These individual constraints each have precedents in the OT literature, but here they are viewed as a group with a common characteristic. As predicted by the Prominence Condition, the markedness constraints with attested M/str counterparts are all constraints that act to enhance perceptual prominence.

This section discusses the relationship that each of the constraints given in (21) has with perceptual prominence. In addition, a formulation is developed for each constraint that explicitly identifies its focus. As discussed in $\$ 2.2 .2$, the constraint focus is important because it determines the strong positions to which a constraint can be meaningfully relativized. (See $\S 2.3 .3$ below for discussion of which positional versions are predicted for each of these constraints.)

\subsubsection{HEAVYO}

One of the most familiar examples of a phonological requirement that holds specifically of a strong position is that seen in the close relationship between stressed syllables and syllable weight: in some languages, stress is attracted to heavy syllables, while in other languages, stressed syllables themselves undergo lengthening. In $\S 3.2 .1$, these two effects are shown to be responses to the same constraint, HEAVYo/ó, which penalizes monomoraic stressed syllables. ${ }^{14}$

(22) HEAvYo/ó For all syllables $x$, if $x$ is a ó, then $x$ dominates $>1$ mora

Some languages choose to satisfy this constraint by altering the stressed syllable (as by lengthening its vowel, or geminating or resyllabifying a following consonant),

14. See the end of this subsection (\$2.3.2.1) for discussion of the relationship between HEAVYo/ó and similar constraints that have been proposed in the literature, STRESS-TOWeIGHT (Prince 1990) and PK-Prom (Prince \& Smolensky 1993; Kenstowicz 1994). 
whereas other languages that satisfy this constraint do so by adjusting the location of the stress so that it falls on a syllable that is intrinsically heavy.

By the Prominence Condition, any markedness constraint that has an $\mathbf{M} / \mathbf{s t r}$ counterpart must be an augmentation constraint, a constraint calling for some property that is perceptually prominent. Heavy syllable weight has long been recognized as a prominent characteristic, partly based on the very relationship between stress and syllable weight just described. In $\$ 2.3 .1$ above, prominence is defined in terms of relative perceptual response; on this basis as well, heavy syllables qualify as perceptually prominent. A bimoraic syllable is longer than a monomoraic one, since it contains either a longer peak (if $\mathrm{CV}$ :) or more segments (if $\mathrm{CV}_{1} \mathrm{~V}_{2}$ or $\mathrm{CVC}$ ) than a monomoraic (CV) syllable. Thus, a bimoraic syllable would give rise to a larger perceptual response than a monomoraic syllable, all else being equal.

There is a question that arises when the constraint HEAVYo/ó is examined in the larger context of a theory of positional augmentation constraints: does it have a non-positional counterpart, HEAVYo? Thus far, M/str constraints-markedness constraints that make specific reference to strong positions-have been viewed as the relativized counterparts of general markedness constraints. That is, all constraints of the form $\mathbf{M} / \mathbf{s t r}$ have been assumed to be formed by the $\mathrm{C} /$ str schema, which combines a general $\mathbf{M}$ constraint and a member of the set of strong positions (§2.2.1.1; §2.2.2). Many M/str constraints, such as ONSET/ $\sigma_{1}$ or $\left[{ }^{*} \mathrm{PEAK} / \mathrm{X}\right] / \sigma$, clearly are positional counterparts of well-attested general $\mathbf{M}$ constraints, in this case ONSET and ${ }^{*}$ PEAK/X. However, evidence for the existence of a general constraint HEAVYo is not as strong.

General HEAVYo would have the following formulation, without the if-then clause that relativizes HEAVYo/ó (22) to stressed syllables (see §2.2.2 above on the formal relationship between positional and general constraints).

HEAVYo For all syllables $x, x$ dominates $>1$ mora

A constraint calling for all syllables to be heavy does have some precedent in the phonological literature. For example, all Dutch non-schwa syllables have been argued to be bimoraic (van der Hulst 1984, 1985; Kager 1990; but cf. Gussenhoven 2000). It has also been proposed that in various Chinese languages, all tone-bearing syllables are bimoraic (Woo 1969; Yip 1980). An OT constraint equivalent to HEAVYo, called QUANTITY, is introduced by Hammond (1997) for his analysis of English vowels, based on Kager's (1990) account of Dutch.

Of course, there are also many languages with only monomoraic syllables (i.e., typical "quantity-insensitive" languages). This fact might seem to indicate that there is a constraint against bimoraic syllables. However, constraints against bimoraic 
vowels, against diphthongs, and against moraic consonants (including coda consonants) are all independently motivated (Rosenthall 1994; Broselow, Chen, \& Huffman 1997). If all these constraints were ranked high in a particular language, their combined effects would allow only monomoraic syllables, without the need for a constraint that specifically bans bimoraic syllables. ${ }^{15}$

Whatever the ultimate status of a general HEAVYo constraint, nothing in the theory of $\mathbf{M} / \mathbf{s t r}$ constraints developed here crucially depends on its existence. If this constraint does not in fact exist, there are two possible explanations for its absence that are consistent with this framework. First, it is possible that HEAVYo as well as HEAVYo/ó is among the set of formally possible constraints that are examined by the constraint filters, but there is some constraint filter that prevents general HEAVYo from being included in CON.

Second, it is conceivable, although less conceptually appealing, that some $\mathbf{M} / \mathbf{s t r}$ constraints are themselves primitives of the model, rather than being constructed compositionally from general $\mathbf{M}$ constraints by the $\mathbf{C} / s t r$ schema. Crucially, the prediction would still be made that such constraints must pass the Prominence Condition, since this filter acts upon all constraints of the form $\mathbf{M} / \mathbf{s t r}$, whether they came from the $\mathbf{C} / s t r$ schema or were somehow independently constructed.

A final point to note in this subsection is that the stressed-syllable version of this constraint, HEAVYo/ó, has a close relationship to several constraints and principles that have previously been introduced in the phonological literature, particularly the Stress-to-Weight Principle (SWP) of Prince (1990), and the constraint PeaK-Prominence (PK-Prom), introduced by Prince \& Smolensky (1993).

The Stress-to-Weight Principle is so named in Prince (1990), although there are several earlier analyses of vowel lengthening and resyllabification under stress that invoke an analogous weight requirement for stressed syllables (e.g., Chierchia 1982; Borowsky, Itô, \& Mester 1984). Prince states this principle as follows.

15. Even if there turns out to be indisputable evidence in favor of a constraint against bimoraic syllables - perhaps as a member of the *STRUCTURE constraint family (Zoll 1993; also Zoll, p.c., cited in Prince \& Smolensky 1993:25) - this does not necessarily mean that HEAVyo does not exist. There are different "dimensions" of markedness, and a given phonological configuration is often seen to violate one markedness constraint while being demanded by another. FTBIN, which requires feet to be binary (Prince 1980; McCarthy \& Prince 1986, 1993ab), is a classic example of a prosodic constraint that is directly antagonistic to *STRUCTURE constraints, in the same way that HEAVYo would be. 
If stressed, then heavy

The SWP requires any stressed syllable to be a heavy, so it is clearly identical to HEAVYo/ó. (The name HEAVYo/ó is nevertheless used here in the interest of maintaining a consistent, transparent "M/str" naming system for positional augmentation constraints.)

The status of the SWP in Prince (1990) is actually somewhat ambiguous. Prince first states it as a logically possible principle that he will nonetheless "specifically deny" has the status of an actual principle (Prince 1990:358). Then, in a footnote, he retracts a bit from this initial claim: "A more even-tempered position would hold that Stress-to-Weight is a principle, but one with a different [i.e., subordinate-JLS] position than Weight-to-Stress in the ranking of rhythmic priorities" (Prince 1990:388, note 6).

Prince's (1990) view of the SWP should be understood in the context of a major goal of that paper, which is to use the Weight-to-Stress Principle (WSP; 'if heavy, then stressed') and considerations of foot form to account for cross-linguistic patterns of iambic lengthening and trochaic shortening. Prince (1990:376) himself observes that the system he has developed is unable to account for the trochaic lengthening that occurs under stress in English and similar languages (cf. West Germanic, addressed in $\$ 3.2 .1 .1$ ). This is precisely the kind of case that the SWP HEAVYo/ó can account for.

Indeed, once the WSP and the SWP (the latter commonly under the guise of PK-РRом; see below) are incorporated into OT where all constraints are violable, it is generally acknowledged that both types of 'principle' or constraint are necessary, in addition to constraints regulating foot form (e.g., Prince \& Smolensky 1993; Walker 1996). ${ }^{16}$

16. Specifically, the WSP is crucially needed for languages that have obligatory stress (primary or secondary) on all heavy syllables, a pattern that cannot be accounted for by the SWP, which would be satisfied if just one of several heavy syllables were to bear stress. However, the SWP is also necessary. It is needed to account for stressed-syllable lengthening effects (particularly in trochaic systems, where no appeal to "uneven iambs" can account for weight gain in stressed syllables); since the WSP would be perfectly satisfied by a light stressed syllable if there were no heavy syllables in a form, it cannot force a stressed syllable to become heavy. See, e.g., Holt (1997) and Gussenhoven (2000), who implement the SWP as an OT constraint to account for stressed-syllable lengthening effects in the Iberian languages and Dutch respectively. Additional discussion of stressed-syllable lengthening is given in \$3.2.1.1. 
The other constraint that is closely related to HEAvYo/ó is PK-PROM, a constraint often invoked to account for unbounded stress systems, in which syllables having certain properties preferentially bear stress. The original formulation of this constraint is given as follows (Prince \& Smolensky 1993:39).

$$
\text { Peak-Prominence (PK-ProM) } \quad \operatorname{Peak}(x) \succ \operatorname{Peak}(y) \text { if }|x|>|y|
$$

This formulation says that "the element $x$ is a better peak than $y$ if the intrinsic prominence of $x$ is greater than that of $y$," where $x$ and $y$ are syllables and "peak" here refers to word peak, i.e., main stress (Prince \& Smolensky 1993:38-9). There is reason to believe, however, that (25) is intended to be an encapsulation of several simpler constraints rather than a formal constraint definition. PK-PROM as given in (25) has the same structure as another constraint provisionally proposed by Prince \& Smolensky (1993:16), HNUC (26), and HNUC is eventually replaced by the *PEAK/X subhierarchy in Prince \& Smolensky's system for reasons to be described below.

$$
\text { Nuclear Harmony Constraint (HNUC) If }|x|>|y| \text { then Nuc/x }>\text { Nuc/y }
$$

This time, $x$ and $y$ are segments, and HNUC says that a segment of higher intrinsic prominence (greater sonority) is a better syllable nucleus than one of lower intrinsic prominence.

In addition to being constraints that evaluate relative prominence, PK-PROM and HNUC are also both what Prince \& Smolensky (1993) call "non-binary constraints," because the marks that they assign to candidates involve not a choice between satisfaction and violation $\left(\checkmark\right.$ versus $\left.{ }^{*}\right)$, but symbols that represent the levels of prominence of the objects that they evaluate $(|\mathrm{a}|$ versus $|\mathrm{i}|$ versus $|\mathrm{n}|$, for example). However, Prince and Smolensky (1993:134) later go on to decompose HNUC into the *PEAK/X subhierarchy, consisting of the universally ranked binary constraints *PEAK/[t] $\gg>\ldots$. . *PEAK/[n] $\gg>\ldots>$. . PEAK/[a] (see also $\S 2.3 .2 .2$ ). They note:

As might be suspected, it will turn out that the work done by a single non-binary constraint like HNUC can also be done by a set (indeed a sub-hierarchy) of binary constraints. (Prince \& Smolensky 1993:81)

Thus, although Prince \& Smolensky (1993) themselves never explicitly reformulate PK-PROM by means of binary constraints, it seems clear that they intend PK-PrOM as an expository convenience, an encapsulation of various binary constraints relating stress and syllable prominence, rather than as an actual single constraint in 
the system. Subsequent researchers who invoke PK-Prom (e.g., Walker 1996) generally do so in the spirit in which it was intended-as an encapsulation.

The relationship of HEAVYo/ó to PK-PrOM becomes apparent once the individual binary constraints that will be needed to replace this encapsulated constraint are considered. As is explicitly noted in most analyses that make use of encapsulated PK-Prom (e.g., Prince \& Smolensky 1993; Walker 1996), the particular property that qualifies as 'prominence' for stress placement varies from language to language, including such characteristics as mora count, vowel sonority, tone, and onset profile (see also Hayes 1995:Ch 7). Thus, it is necessary to recognize distinct constraints or constraint subhierarchies, each of which requires stressed syllables to have one of these distinct dimensions of prominence. ${ }^{17}$ The subcomponent of PK-PROM that relates stressed syllables to syllable weight is a constraint that says, "Stressed syllables are heavy"; that is, it is none other than HEAvYo/ó. Thus, HEAVYo/ó is "part of" PK-PrOM. (Other constraints that enforce stress-prominence interactions, and so are needed to help decompose PK-PROM, include HTONE/ó, [*PEAK/X]/ó, ONSET/ó, and [*ONSET/X]/ó. These constraints, along with HEAVYo/ó, are exemplified and further discussed in §3.2. The general $\mathbf{M}$ constraints from which these $\mathbf{M} / \sigma^{\prime}$ constraints are built are presented later in the current section, §2.3.2.)

In summary, HEAvYo/ó is equivalent to the Stress-to-Weight principle (Prince 1990), and it is one of the individual constraints that is encapsulated by PK-PROM (Prince \& Smolensky 1993). The SWP has typically been invoked in cases where stressed syllables are required to become heavy, whereas PK-PROM has generally been used to explain the attraction of stress to syllables that are already heavy. Recognizing that these two are in fact the same constraint means that two distinct types of phonological behavior-stressed-syllable lengthening and the attraction of stress to heavy syllables - can be directly related. (See $\$ 3.2$ for further discussion, and for extensions of this approach to the relationship between stress and other dimensions of prominence beyond syllable weight.)

17. Previous analyses that expand PK-PROM in order to account more directly for the kinds of prominence-sensitive stress systems that are attested cross-linguistically include Kenstowicz (1994), which focuses specifically on peak prominence related to vowel sonority (see the discussion of *PEAK/X in \$2.3.2.2); de Lacy (1997), which replaces PK-PROM with a number of constraints sensitive to prosodic branchingness (explicitly rejecting mora count as relevant), and de Lacy (1999), which explores the relationship between stress peaks and troughs on the one hand and high and low tone on the other (see also the discussion of HTONE in \$2.3.2.5). 


\subsubsection{2 *PEAK/X}

Another phonological requirement that is observed in some languages to hold of strong positions (in particular, of stressed syllables and long vowels) is the requirement that syllable peaks in those positions be high in sonority.

The general (non-positional) markedness constraints responsible for demanding high-sonority syllable peaks are the members of the *PEAK/X subhierarchy (Prince \& Smolensky 1993). This constraint subhierarchy is based on the segmental sonority scale; the members of the subhierarchy can each be given the explicit formulation in (27).

$*$ PEAK/X For every segment $a$ that is the head of some syllable $x$,
$|a|>\mathrm{X}$

where $|y|$ is the sonority of segment $y$

$\mathrm{X}$ is a particular step on the segmental sonority scale

Prince and Smolensky (1993:136) propose that the members of the *PEAK/X subhierarchy are in a universally fixed ranking that is determined by harmonic alignment. That is, syllable peak (nucleus) and syllable margin (onset or coda) form a prominence scale Peak > Margin ('Peak is more prominent than Margin'), and the steps of the segmental sonority hierarchy also form a prominence scale $a>\ldots n$ $>\ldots>t$. Harmonic alignment is an operation that combines two such prominence scales into constraint subhierarchies with universally fixed rankings. Here, since Peak is more prominent than Margin, Peak preferentially co-occurs with the prominent end of the segmental sonority scale and disprefers the non-prominent end, giving rise to the ${ }^{*} \mathrm{PEAK} / \mathrm{X}$ subhierarchy, ${ }^{*} \mathrm{PEAK} / \mathrm{T} \gg \ldots . .>{ }^{*} \mathrm{PEAK} / \mathrm{NAS}$ $>>$.. > $>$ *PEAK/LOWV. ${ }^{18}$

Because the members of the *PEAK/X subhierarchy demand that a syllable peak be high in sonority, these constraints qualify as augmentation constraints. The precise definition of sonority is somewhat controversial (see Parker 2002 for a review of the relevant literature), but many proposals involve a property related to acoustic intensity, amplitude, clear formant structures, and perceptual salience

18. Prince \& Smolensky (1993:134) also propose a *MARGIN/X subhierarchy from harmonic alignment, *MARGIN/LOWV $>$. . . > *MARGIN/NAS $\gg . . .>*$ MARGIN/T, in which less prominent Margin preferentially co-occurs with the less prominent end of the segmental sonority scale. See $\$ 2.3 .2 .3 .2$ for discussion of ${ }^{*}$ ONSET/X, which is based on *MARgIN/X. 
(representative works include Sievers 1881, Bloomfield 1933, Bloch \& Trager 1942, Selkirk 1984, Keating 1983, Clements 1990, Pierrehumbert \& Talkin 1992, Lavoie 2000, Parker 2002).

The sonority scale adopted here, from which the identity of the individual members of the *PEAK/X subhierarchy is determined, is given in (28) (see Sievers 1881, Jespersen 1904, Steriade 1982, Selkirk 1984, and the "consonantal strength scale" of Vennemann 1988). ${ }^{19}$

Sonority scale

$\begin{array}{ll}\text { high sonority } & \text { low vowels } \\ & \text { mid vowels } \\ & \text { high vowels/glides } \\ & \text { rhotics } \\ & \text { laterals } \\ & \text { nasals } \\ & \text { voiced obstruents } \\ & \text { voiceless obstruents }\end{array}$

This version of the sonority scale is partitioned finely enough to account for the onset sonority- and peak sonority-sensitive phenomena encountered in the case studies in chapters 3 and 4 . In addition to sonority divisions among the major

19. Although there is much about the sonority scale that is widely accepted, there are a few points of contention in the literature, stemming mostly from cases where one language or one phonological phenomenon appears to treat segment class $A$ as higher in sonority than segment class $B$, while a different language or a different phenomenon seems to treat $B$ as higher in sonority than $A$. This kind of problem-where near-universal tendencies are overridden in particular languages or in particular circumstances-is often amenable to an OT solution in terms of constraint conflict. It is therefore likely that these apparent "sonority reversals" can be accounted for by ranking some other constraint above those constraints concerned with sonority; constraint domination thus leads to "exceptional" behavior from the point of view of sonority.

One such controversy is the question of whether the glottal segments [h, ?] are low in sonority, like obstruents, or high in sonority, like glides. In the discussion of HAVECPLACE in \$2.3.2.4, it is argued that these segments are classified as obstruents for the purposes of sonority, and that occasions when they behave differently from obstruents are caused by their lack of a supralaryngeal place specification rather than by anything to do with sonority. Thus, in a system in which constraints are violable, we are not forced $a$ priori to abandon a proposed step or division of the segmental sonority scale simply because it is not universally observed. 
classes of segments, vocoids > liquids > nasals > obstruents (Clements 1990; Zec 1988), a few additional divisions are also recognized. Namely, the class of liquids is divided into rhotics > laterals; the class of obstruents is divided into voiced > voiceless, and the class of vocoids is divided into low vowels $>$ mid vowels $>$ high vowels/glides.

Evidence for these finer sonority distinctions comes from phonological patterns in a number of languages and from phonetic characteristics of the sounds in question. First, consider the rhotic $>$ lateral division. Rhotics often pattern with glides rather than with laterals in sonority-sensitive phenomena, showing that rhotics are higher in sonority than laterals. In Icelandic, for example, the rhotic and the glides [j, w], but not the lateral, can be syllabified together with a preceding obstruent into an onset cluster word-medially (Einarsson 1949, cited in Devine \& Stephens 1994). Also, rhotics are more compatible with syllable-peak status than laterals in several languages. Zec (1995) provides two examples: in Gonja, the single liquid phoneme is realized as [ $\left.\mathrm{r}^{\mathrm{w}}\right]$ when syllabic and as [1] when not syllabic, and in Serbo-Croatian, [ $r$ can be syllabic but [1] cannot. (See also the discussion of English in \$3.2.1.3.) From a perceptual standpoint, laterals are like nasals in that both have abrupt spectral discontinuities in the transition to an adjacent vowel, but rhotics are like glides in lacking such discontinuities (J. Kingston, p.c.; see EspyWilson 1992 for the specific case of [1] versus [ $\mathrm{r} \mathrm{j} \mathrm{w}$ ] in American English).$^{20}$ If rhotics share acoustic characteristics with glides, and laterals with nasals, then this supports the claim that rhotics are higher in sonority than laterals.

There is also a phonetic basis for the proposed sonority distinction between voiced and voiceless obstruents. Stevens \& Keyser (1989) argue that since the canonical auditory characteristic of a sonorant is the presence of low-frequency energy in the signal, voiced obstruents, which have low-frequency energy contributed by phonation, are auditorily more like sonorants than voiceless obstruents are. Phonologically, voiced obstruents pattern as though they are higher in sonority than voiceless obstruents (see the discussion of Pirahã in §3.2.2.4). Also, the voicing of obstruents is a common form of synchronic and diachronic lenition, and non-place-related lenition is often defined as a change that causes an increase in the sonority of a segment (Lavoie 2000 and references therein). ${ }^{21}$

20. Kingston (p.c.) notes that despite the articulatory diversity within the class of rhotics, most or all rhotics, from retroflex approximants to coronal and uvular trills, seem to share this property.

21. Languages apparently differ in which divisions among the class of obstruents are most phonologically relevant. In some languages, the stop/fricative distinction is primary and the voiceless/voiced distinction is secondary; that is, all fricatives are higher in sonority than all stops, with a voiced > voiceless division inside each group (as in Imdlawn Tashlhiyt Berber; 
Finally, many of the segmental sonority scales that have been proposed, including those of Jespersen (1904), Selkirk (1984), and Vennemann (1988), recognize distinctions among the vowels according to height. Phonetically, the lower a vowel, the greater the degree of jaw opening, which results in more airflow and higher acoustic intensity at low frequencies (Keating 1983). Phonological motivation for recognizing this distinction comes from languages with sonoritysensitive phenomena that distinguish among vowel heights. For example, Kobon (Kenstowicz 1994, who attributes the example to "Davies 1981") assigns stress to low vowels when possible, to mid vowels when no low vowels are available, and to high vowels when no low or mid vowels are available. ${ }^{22}$

Dell \& Elmedlaoui 1985, 1988). But in Pirahã (\$4.2.3.2), the voiceless/voiced distinction is primary-all voiced obstruents are higher in sonority than all voiceless obstruents, including the voiceless fricatives.

Zec (1988:94) tries to avoid the problem of conflicting subdivisions within the class of obstruents by proposing that the feature [voice] cannot be used to define a sonority class. Zec makes this choice because she has already proposed that [continuant] can be used to make sonority distinctions, and she wants to make the above kind of cross-cutting classification impossible. However, the two primary ways of dividing the class of obstruents are both attested, in IT Berber and Pirahã respectively. Moreover, given that there is an acoustic difference between voiced and voiceless obstruents of the sort that is relevant for sonority - greater low-frequency energy for voiced than for voiceless obstruents-it seems well motivated to divide the class of obstruents according to [voice]. Perhaps the phonologically different behavior of stops and fricatives, in languages that do distinguish the two, is the result of some constraint related to a property other than sonority, which can be ranked differently in different languages (similar to the approach taken in \$2.3.2.4 below for glottal consonants). For now, since the status of stops versus continuants is not crucial for any of the positional augmentation examples considered in chapters 3 and 4 , this question is pursued no further. See also Morén (1999) for arguments that obstruent voicing is relevant for sonority.

22. It has sometimes been proposed that central or reduced vowels, such as schwa, also differ from other vowels in terms of sonority. For example, reduced vowels are placed on the sonority scale below high vowels by Kenstowicz (1994), because many languages prefer high vowels over schwa for stress assignment-including Kobon, mentioned above, which prefers high vowels to $[\dot{+}$, ə] , and Mokshan Mordwin (\$4.2.5.2), which prefers mid vowels to schwa as well as to high vowels. However, admitting the constraint *PEAK/REDV, against reduced vowels, into the ${ }^{*} \mathrm{PEAK} / \mathrm{X}$ subhierarchy has an undesired consequence. Since the ${ }^{*}$ PEAK/X subhierarchy and the *ONSET/X subhierarchy (see §2.3.2.3.2 below) are both built from the sonority hierarchy, then these two subhierarchies must contain the same steps. But *PEAK/HIGHV $>$ *PEAK/REDV implies *ONSET/REDV $>>* O N S E T / G L I(=H I G H V)$-namely, that reduced vowels make better onsets than glides do. This certainly does not seem to be the case. I therefore make a provisional proposal, analogous to that for the glottal consonants 
In conclusion, based on the segmental sonority scale in (28), the *PEAK/X subhierarchy is composed of the following individual constraints (29), each of which has the formulation in (30). ( $T$ and $D$ in (29) are abbreviations for "voiceless obstruents" and "voiced obstruents" respectively.)

The *PEAK/X subhierarchy

$$
\begin{aligned}
& \text { *PEAK/T } \gg \text { *PEAK/D } \gg \text { *PEAK/NAS } \gg \text { *PEAK/LAT } \gg \text { *PEAK/RHO } \gg \\
& * \mathrm{PEAK} / \mathrm{HIGHV} \gg \text { } \gg \text { PEAK/MIDV }>>\text { *PEAK/LOWV }
\end{aligned}
$$

$$
\begin{aligned}
& { }^{*} \mathrm{PEAK} / \mathrm{X} \text { For every segment } a \text { that is the head of some syllable } x,|a|>\mathrm{X} \\
& \text { where }|y| \text { is the sonority of segment } y \\
& \mathrm{X} \text { is a particular step on the segmental sonority scale }
\end{aligned}
$$

Further confirmation of the sonority divisions within major classes that are included in (28) and (29) is provided by several of the languages discussed in chapters 3 and 4. For rhotic $>$ lateral, see English syllabic sonorants (\$3.2.1.3) and word-initial liquids in Sestu Campidanian Sardinian and Mbabaram (§4.2.1.2). For voiced obstruents $>$ voiceless obstruents, see Pirahã (\$3.2.2.4). For sonority divisions among vowels, see Zabiče Slovene and Mokshan Mordwin (§3.2.1.3) and Yawelmani (\$3.3).

\subsubsection{ONSET and *ONSET/X}

Two further phonological requirements that are empirically observed to apply specifically to strong positions (in this case, stressed syllables and initial syllables)

[h, ?] (see §2.3.2.4). Namely, reduced vowels differ from other vowels not in terms of sonority, but in terms of a separate dimension that is not related to the segmental sonority scale, and it is a constraint disfavoring that particular characteristic of reduced vowels that causes stress to avoid them.

If reduced vowels differ from full vowels in lacking vowel-place features, then the constraint in question might be HAVEVPLACE. However, Hammond (1997) argues that schwa in English is not placeless, but rather fails to sponsor a mora. Furthermore, there are languages, like Kobon, where there is a phonological contrast between different reduced vowels (both of which are avoided for stress). So lack of place features may not be what sets reduced vowels apart. 
are the requirement that syllables in strong positions have onsets and the requirement that syllable onsets in strong positions be low in sonority.

For many of the strong-position markedness requirements discussed here, the Prominence Condition is clearly met; it is widely accepted that characteristics such as syllable weight (\$2.3.2.1), high syllable-peak sonority (\$2.3.2.2), and high tone (see $\S 2.3 .2 .5$ below) are perceptually prominent. However, the relationship between the Prominence Condition and constraints calling for onsets (ONSET) or lowsonority onsets (the *ONSET/X subhierarchy) is less obvious- $-\mathrm{CV}$ syllables are less marked than onsetless syllables, and low-sonority onsets are less marked than highsonority onsets, but these facts alone do not entail that syllables with onsets or lowsonority onsets are more perceptually prominent than other syllables. (As discussed in \$2.3.2.2 above, lower sonority means lower prominence, so onset consonants themselves are actually less perceptually prominent when ${ }^{*}$ ONSET/X constraints are satisfied.)

This section presents evidence from neural response patterns that the presence of an onset, and specifically a low-sonority onset, does in fact enhance the perceptual response to a syllable $(\S 2.3 .2 .3 .1)$. Because this is the case, the Prominence Condition correctly predicts that there should be $\mathbf{M} / \mathbf{s t r}$ counterparts of ONSET and the *ONSET/X subhierarchy. Explicit formulations for these constraints are given in $\S 2.3 .2 .3 .2$ and $\$ 2.3 .2 .3 .3$ respectively.

\subsection{On the perceptual prominence of syllables with (low-sonority) onsets}

Given a constant auditory stimulus such as a tone or a vowel-like sound, auditorynerve fibers do not discharge at a constant rate. There is an initial higher response rate when the stimulus begins, followed by a decay in response rate that is known as adaptation. Adaptation has a physiological origin, because it is apparently caused by depletion of the neurotransmitter that stimulates the auditory-nerve fibers (R. Smith 1979). However, it also plays a role in speech perception:

[A]daptation enhances spectral contrast between successive speech segments.... [A] fiber adapted by stimulus components close to its CF [characteristic frequency] is less responsive to subsequent stimuli that share spectral components with the adapting sound. On the other hand, stimuli with novel spectral components stimulate 'fresh,' unadapted fibers, thereby producing an enhanced response. (Delgutte 1997:510) 
Therefore, interspersing consonants (syllable onsets) between vowels gives the peripheral auditory system time to recover from adaptation, allowing enhanced response for each new vowel (syllable) in the string, as seen in (31).

(31) Neural response to synthesized [ada] (adapted from Delgutte 1997:531)

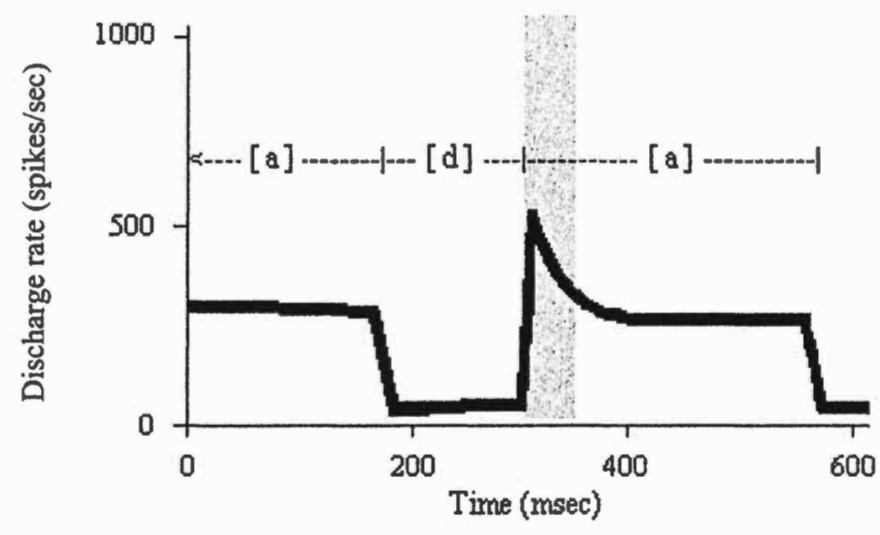

This figure is adapted from a post-stimulus time histogram for a high-spontaneous nerve fiber $(\mathrm{CF}=1800 \mathrm{~Hz})$. The stimulus is a synthesized sequence [ada] (with equal intensity in both syllables). The shaded bar indicates the time interval occupied by the $\mathrm{CV}$ formant transitions, so its left edge marks the point of consonantal release.

At the time of release into the second [a], this nerve fiber shows some recovery from adaptation. The response rate there, at approximately 500 spikes per second, is larger than the response rate observed where adaptation has set in (i.e., the portion of the neural response to the first [a] that is shown, ${ }^{23}$ and the response to the second [a] after about $350 \mathrm{~ms}$ ).

Furthermore, if $\mathrm{CV}$ syllables are more prominent than V syllables because the onset consonant provides a contrast to the vowel (thereby allowing the peripheral auditory system time to recover from adaptation), it follows that syllables with lowsonority onsets are even more prominent than syllables with high-sonority onsets.

23. Responses to other stimuli by the same nerve fiber indicate that the initial response rate for the first [a], before adaptation, was probably between 500 and $700 \mathrm{sp} / \mathrm{sec}$ (Delgutte 1997:531). 
A low-sonority onset such as a voiceless stop is maximally distinct from a vowel, and so would provide the best opportunity for recovery from adaptation. ${ }^{24}$

In summary, there is evidence from neural response patterns that syllables with onsets are more perceptually prominent than syllables without onsets, and further, that syllables with low-sonority onsets are more perceptually prominent than syllables with high-sonority onsets. As a result, ONSET and the *ONSET/X subhierarchy qualify as prominence-enhancing constraints, and $\mathbf{M} / \mathbf{s t r}$ versions of these constraints are correctly predicted to pass the Prominence Condition.

Formulations of these constraints, and discussion of the motivation for the formulations, are given in the following two subsections.

\subsection{ONSET}

The constraint ONSET (Prince \& Smolensky 1993), which requires syllables to have onsets, is the optimality-theoretic successor to ideas such as CV core syllable formation, onset maximization, and the Onset Principle (e.g., Clements \& Keyser 1983; Itô 1986, 1989; Kahn 1976; Selkirk 1982; Steriade 1982). As described above, the substantive motivation for this constraint is the fact that recovery from neural adaptation is facilitated when vowels are separated by consonants.

The formulation of ONSET adopted here is given in (32).

$$
\text { ONSET For all syllables } x, a \neq b
$$

where $a$ is the leftmost segment dominated by $x$

$b$ is the head of $x$

According to this formulation, ONSET requires that the head of a syllable not be the leftmost segment in the syllable. This version of the constraint is similar, but not identical, to the versions of ONSET proposed in Prince \& Smolensky (1993) and McCarthy \& Prince (1993a) (33).

24. Discussion of the importance of spectral discontinuities in speech perception can also be found in, e.g., Stevens (1989) and Ohala (1992). Warner (1998) uses facts about adaptation, among other evidence, to argue for the importance of dynamic cues over static cues in speech perception. See also Silverman (1995) for another application of neural response patterns to phonological markedness; Silverman investigates a phenomenon separate from those considered here, namely, the relative markedness of different subsegmental orderings of features such as aspiration, but he gives detailed arguments for his phonological proposal based on neural response patterns. 
(33) Previous formulations of ONSET

(a) Every syllable has an Onset [node] (Prince \& Smolensky 1993:25)

(b) Align-L (o, C) (McCarthy \& Prince 1993a:101)

('The left edge of every syllable is aligned with the left edge of some consonant')

The current formulation (32) differs from the previous formulations shown in (33) in two ways. First, (32) does not require the onset to appear in a particular syllabic position (cf. (33a)). As a result, ONSET is satisfied by the presence of either a true structural onset (34a) or what is termed here a nuclear onglide (34b) (see $\S 2.3 .2 .3 .3$ immediately below, and also $\$ 4.2 .1 .2 .4$, for discussion of the significance of this distinction).

(a) True onset

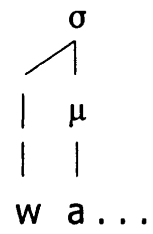

(b) Nuclear onglide

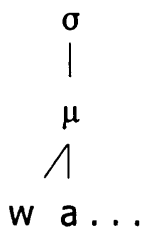

Second, ONSET as formulated in (32) does not make reference to the consonantal/vocalic status of segments (cf. (33b)). Evidence from Imdlawn Tashlhiyt Berber (Prince \& Smolensky 1993, based on work by Dell \& Elmedlaoui $1985,1988,1992)$ shows that ONSET is not sensitive to major-class features or other aspects of segmental sonority. ONSET is never violated in IT Berber (except in steminitial position, where onset epenthesis would disrupt stem-to-PrWd alignment). Crucially, the drive to satisfy ONSET is able to force syllabifications like [.wL.] (where a capital letter indicates nuclear status) in [hA.wL.tN] 'make them (m.) plentiful'; clearly, to syllabify these two segments as [.Ul.] would provide a better nucleus, and the only explanation for why that syllabification is not chosen is that the actual syllabification [.wL.] satisfies ONSET (Prince \& Smolensky 1993:17). And yet in this syllable, the leftmost segment, [w], is not lower in sonority than the nucleus, [1]. So what matters for satisfaction of ONSET is simply that there be a segment to the left of the head; the relative sonority of that segment with respect to the head is not important. 


\subsubsection{3* ONSET/X}

Another constraint, or rather set of constraints, that has its substantive basis in the perceptual advantage gained by interspersing low-sonority elements between vowels is the * ONSET/X subhierarchy, formulated as in (35).

*ONSET/X

For every segment $a$ that is the leftmost pre-moraic segment ${ }^{25}$ of some syllable $x,|a|<\mathrm{X}$

where $|y|$ is the sonority of segment $y$

$\mathrm{X}$ is a particular step on the segmental sonority scale

The *ONSET/X subhierarchy is a refinement of the *MARGIN/X subhierarchy of Prince \& Smolensky (1993), which they state as follows.

*MARGIN/X subhierarchy (Prince \& Smolensky 1993:135)

${ }^{*} \mathrm{M} / a \gg{ }^{*} \mathrm{M} / i \gg, \ldots \gg * \mathrm{M} / t$

Just as with the *PEAK/X subhierarchy (see §2.3.2.2 above), Prince and Smolensky (1993:136) generate the *MARGIN/X subhierarchy through the harmonic alignment of two prominence scales: the syllable-position prominence scale Peak > Margin and the segmental sonority scale. Since a syllable margin is less intrinsically prominent than a syllable peak, Margin preferentially co-occurs with the nonprominent end of the segmental sonority scale.

Although the *ONSET/X subhierarchy implemented here is based on the *MARgIN/X hierarchy of Prince \& Smolensky (1993), there are significant differences. As a comparison of the constraint names suggests, *ONSET/X constraints specifically enforce sonority requirements on syllable onsets, not on all 'margin' or non-peak positions, which would include codas and nuclear onglides as well. Furthermore, if a language allows onset clusters, only the leftmost segment of the onset is relevant for ${ }^{*}$ ONSET/X constraints. These points are now addressed in turn.

The reason for excluding codas from the scope of this constraint subhierarchy is that, in general, the sonority restrictions that hold of onsets are not the same as those that hold of codas. Onsets are indeed preferentially low in sonority, as

25. The term 'pre-moraic segment' designates a segment that precedes a tautosyllabic mora and is not itself dominated by a mora. 
evidenced, for example, by Sanskrit reduplication (Steriade 1982), in which the lowest-sonority member of an onset cluster is copied into the reduplicant. However, many languages show a preference for high-sonority coda consonants (e.g., Hooper 1976; Zec 1988, 1995; Clements 1990). Prince \& Smolensky (1993:162) themselves note that "the most Harmonic codas are generally regarded to be those which are most sonorous." Therefore, instead of grouping both onsets and codas together into a *MARGIN/X subhierarchy, which incorrectly predicts that lowsonority segments are preferred in codas as well as in onsets, it seems advisable to allow coda sonority to be regulated separately, by a different constraint or constraint subhierarchy. ${ }^{26}$

The reason for excluding nuclear onglides (34b)-pre-peak glides that are dominated by a mora rather than being immediately dominated by the syllable node - from the scope of * ONSET/X is because empirically, *ONSET/GLI is only violated by glides that are true structural onsets. Syllable-initial glides do not incur

26. Perhaps *PEAK/X could be reinterpreted as *RIME/X, or *MORAIC-SEG/X (in the spirit of Zec 1988, 1995), so that it governs both nuclear and coda segments. The universal tendency for nuclei to be higher in sonority than codas could then be accounted for with constraints that call for the head mora of a syllable to be associated with the highest-sonority element. An approach that utilizes *MORAIC-SEG/X seems especially promising, since it could distinguish moraic codas, which are preferentially high in sonority, from nonmoraic syllable appendices, which are often voiceless (coronal) obstruents.

Prince \& Smolensky (1993:162-3) sketch a few proposals that maintain the notion 'margin' as a cover term for onsets and codas. They suggest that either codas, being as it were in the margin of the rime, are subject to both Peak and Margin constraints, or there is a *RIME/X subhierarchy such that nuclei are subject to Peak and Rime constraints, codas to Margin and Rime constraints, and onsets to Margin constraints only.

Another way to formally model the greater preference for low sonority in onsets than codas would be to view the *ONSET/X subhierarchy itself as a case of positional augmentation, namely, as *MARGIN/X relativized to the strong position onset-leaving codas subject only to general *MARGIN/X (de Lacy 2000). With this system, it would be possible to generate a language that requires onsets to be lower in sonority than codas. However, there are two reasons why this approach is not taken here. First, as the case studies in chapters 3 and 4 show, ${ }^{*}$ ONSET/X itself can be relativized to the strong positions stressed syllable and initial syllable. If *ONSET/X were already a positional version of general *MARGIN/X, we would be forced to recognize the existence of "doubly positional" constraints. Second, it is not simply that onsets have a stronger tendency toward low sonority than codas do; rather, as Zec $(1988,1995)$ shows, there is an independent preference for codas to be high in sonority. This insight cannot be captured if general *MARGIN/X is the only sonority-related constraint governing codas. 
violations of *ONSET/GLI if they are affiliated with a mora. See $\$ 4.2 .1 .2$, especially $\S 4.2 .1 .2 .4$, for discussion and exemplification.

Finally, the restriction of *ONSET/X constraints to the leftmost onset segment predicts that languages with onset clusters will tolerate high-sonority onset segments if they are not initial in the syllable. In fact, the Sestu dialect of Campidanian Sardinian (\$4.2.1.2.1) does tolerate stop-rhotic onset clusters in initial syllables, as in [tro.nu] 'thunder,' even though simple rhotic onsets are prohibited in this position.

The ${ }^{*}$ ONSET/X subhierarchy is based on the segmental sonority scale; this means that just as for ${ }^{*} \mathrm{PEAK} / \mathrm{X}$, the identity of the individual constraints in this subhierarchy is determined by the identity of the steps of the sonority scale that is to be adopted. Given the sonority scale motivated in $\$ 2.3 .2 .2$ above, shown in (28) and repeated here in (37), the members of the ${ }^{*}$ ONSET/X subhierarchy are as in (38). (Again, $D$ and $T$ are abbreviations for "voiced obstruents" and "voiceless obstruents" respectively.)

Sonority scale

$\begin{array}{ll}\text { high sonority } & \text { low vowels } \\ & \text { mid vowels } \\ & \text { high vowels (glides) } \\ & \text { rhotics } \\ & \text { laterals } \\ & \text { nasals } \\ & \text { voiced obstruents } \\ & \text { voiceless obstruents }\end{array}$

The * ONSET/X subhierarchy

*ONSET/LOWV $>>$ *ONSET/MidV $>$ * ONSET/GLI ${ }^{27}>$ > ONSET/RHO $>>$ *ONSET/LAT $>$ * ONSET/NAS $>$ * ONSET/D $>$ * ONSET/T

Thus, ONSET and the members of the * ONSET/X subhierarchy are markedness constraints that are functionally grounded in a preference for demarcating vowels in the speech string with spectrally contrasting, low-sonority elements; furthermore,

27. The constraints *ONSET/GLI and *PEAK/HIGHV refer to the same class of segments, since the only difference between a high vowel and a glide is the segment's syllabic status. Thus, as an onset, such a segment will be a glide, and as a peak it will be a high vowel. 
both ONSET and *ONSET/X qualify as augmentation constraints, because syllables with onsets, or with low-sonority onsets, are more perceptually prominent than other syllables. However, despite these basic similarities, there are important formal differences between ONSET and *ONSET/X. ONSET cannot simply be seen as another member of the *ONSET/X subhierarchy for two reasons: the ranking of ONSET is not fixed with respect to the members of the *ONSET/X subhierarchy, and moreover ONSET and *ONSET/X make reference to different aspects of syllable structure.

It might seem to be possible to recast ONSET as "*ONSET/Ø," a constraint against a "zero" onset. Such a constraint would have to dominate all the other constraints in the *ONSET/X subhierarchy, since a "zero" onset would be even less distinct from the syllable peak than a glide onset would be, and the members of the *ONSET/X subhierarchy are in a universally fixed ranking based on the sonority scale. However, there is evidence from Niuafo'ou that the stressed-syllable version of ONSET is in fact dominated by the stressed-syllable version of *ONSET/GLI: stressed syllables sometimes lack onsets altogether, but they never have glide onsets (§3.2.2.3). Thus, ONSET must be formally distinct from the *ONSET/X subhierarchy, since its ranking with respect to the members of ${ }^{*}$ ONSET/X is not universally fixed.

Additionally, ONSET and *ONSET/X are sensitive to different aspects of subsyllabic structure. ONSET simply requires that the head segment not be leftmost in a syllable; as noted in $\$ 2.3 .2 .3 .2$ above, this means that ONSET is satisfied by a true consonantal onset, dominated by the syllable node (39a), as well as by a nuclear onglide, dominated by a mora (39b). On the other hand, ${ }^{*}$ ONSET/X is sensitive only to segments syllabified as true onsets (39a).

(a) True onset

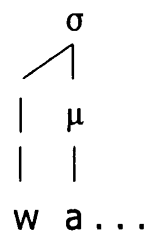

(b) Nuclear onglide

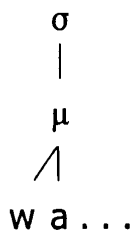

Evidence for this difference between ONSET and *ONSET/X comes from Guugu Yimidhirr and Pitta-Pitta (\$4.2.1.2). In these languages, the initial-syllable versions of *ONSET/GLI, *ONSET/RHO, and *ONSET/LAT are all high-ranking (\$4.2.1.2.2), but word-initial glides do nevertheless appear; this shows that the word-initial glides are syllabified as nuclear onglides and, crucially, that nuclear onglides do not violate *ONSET/GLI (see $\S 4.2 .1 .2 .4$ for discussion). On the other hand, onsetless syllables are not tolerated in these languages, which shows that ONSET is undominated (\$4.2.1.2.2). The fact that some words in these languages begin with nuclear onglides means that, along with true structural onsets, nuclear onglides must 
also be visible to, and satisfy, ONSET. Thus, as reflected in the constraint formulations given above, ONSET is sensitive to the presence of any pre-peak segment, regardless of its syllabic position, but *ONSET/X constraints are relevant only for true, non-moraic onsets.

In conclusion, despite their functional similarity, ONSET and *ONSET/X are formally distinct. This fact is further evidence that although the phonological system is significantly shaped by substantive factors, it is fundamentally an abstract formal system.

\subsubsection{HAVECPLACE}

The requirement that a consonant have a supralaryngeal place specification is another of the requirements that is seen to hold specifically of material in a strong position. Parker (2000) demonstrates that a positional version of this constraint, relativized to the syllable onset, is active in Chamicuro, where the glottal consonants [h, ?] appear as codas but not as onsets (see §3.4).

The general version of this constraint is given in (40).

HaveCPlace For all consonants $x, x$ has a supralaryngeal Place specification

Evidence for general HAVECPLACE can be found in languages that completely lack glottal segments in their inventory, as in many Romance and Australian languages. This constraint is also needed to account for why glottals and pharyngeals, while less marked even than coronals on the universal Place markedness hierarchy *LAB, *DORS $>{ }^{*}$ COR $>$ *PHAR (Lombardi 2001), are sometimes dispreferred as epenthetic consonants (as in Axininca Campa, where [ $t$ ] is the general epenthetic consonant; Payne 1981).

The existence of the constraint HAVECPLACE, and its positional versions, also provides a solution to the apparent variable sonority of glottals. As noted in §2.3.2.2 above, the glottal segments [h, ?] sometimes pattern with obstruents, and other times with high-sonority segments. In Pirahã (§3.2.2.4), for example, [h, ?] pattern with the class of voiceless obstruents, being preferred over voiced obstruents as onsets in stressed syllables. On the other hand, [h, ?] are sometimes derived from other consonants as the outcome of historical change; such diachronic changes are often classified together with cases of lenition (e.g., obstruent voicing or approximantization), so by association the glottals are sometimes categorized as highsonority segments. Indeed, in Chomsky \& Halle (1968), [h, ?] are considered to be [-consonantal] because they have no oral constriction. Along these lines, Parker 
(2000) observes that the ban on glottal onsets in Chamicuro ( $\$ 3.4)$ could be explained by an extension of the *ONSET/X subhierarchy if glottals are the highest sonority consonants. Nevertheless, the Pirahã facts, in which the glottal segments $[\mathrm{h}, ?]$ are preferred as onsets to stressed syllables even over such relatively lowsonority segments as $[\mathrm{b}, \mathrm{g}]$, cannot be reconciled with the proposal that glottals are high in sonority. The solution to this problem lies in recognizing the independence of sonority and supralaryngeal place. Namely, glottal [h, ?] (and pharyngeal obstruents) are different from other obstruents not in sonority, but only because they lack a supralaryngeal place specification, violating HAVECPLACE. Historical change resulting in glottals is debuccalization, not an increase in sonority. The rejection of glottals from certain structural positions, as in Chamicuro, is related not to sonority, but to place.

The theory of $\mathbf{M} / \mathbf{s t r}$ constraints proposed here entails that, because HAVECPLACE has an M/str counterpart, it must be an augmentation constraint. This is in fact the case. According to Stevens (1971), the glottal segments [h, ?] differ from other consonants in lacking an area of rapid spectral change at the $\mathrm{CV}$ transition point. This is because, with no oral constriction in the consonant, the degree of change in the shape of the oral tract from consonant to vowel is very much smaller than for other consonants. Warner (1998), expanding upon work by Stevens (1971 et seq.), Furui (1986), and others, proposes that periods of rapid change in the speech signal are the most important in speech perception, carrying a large proportion of the information content of the signal; one reason for the importance of such "dynamic cues" is that as the spectral shape of the signal changes, new (unadapted) populations of auditory-nerve fibers respond, as discussed in $\$ 2.3 .2 .3 .1$ above. Given Warner's (1998) proposal about the importance of dynamic cues in perception, consonants with a supralaryngeal place specification, which have greater dynamic cues at a CV transition, are more perceptually salient than [h, ?]. Thus, HAVECPLACE is correctly predicted to pass the Prominence Condition and to form legitimate $\mathbf{M} / \mathbf{s t r}$ constraints.

\subsubsection{HTONE}

Another attested M/str constraint is HTONE/ó, which requires stressed syllables to have a tone-bearing unit (TBU) ${ }^{28}$ that is associated with a high $(\mathrm{H})$ tone $(\S 3.2 .1 .2)$.

28. This particular formulation of the constraint allows for the choice of syllable or mora as the prosodic category that serves as TBU to be made on a language-specific basis (under OT, by means of different rankings of constraints regulating associations between tones and 
(41) HTONE/ó

For all syllables $x$, if $\mathrm{x}$ is a $\sigma$, then a tone-bearing unit associated with $x$ bears high tone

It is often proposed that $\mathrm{H}$ tone is more prominent than mid (M) and low (L) tone. See for example Meredith (1990), Jiang-King (1996), de Lacy (1999), and the references cited in these works for discussion of phonologically relevant prominence hierarchies among tones, often described as analogous to the segmental sonority scale. In terms of perceptual prominence, it has been shown that the neural response to a higher-frequency stimulus is more frequency-specific that the neural response to a lower-frequency stimulus (Kluender, Lotto, \& Jenison 1995), a factor which may help make higher tones more perceptually salient. Also, since higherfrequency stimuli by definition have more periods per unit time, they have a greater integrated signal intensity than lower-frequency stimuli (J. Kingston, p.c.).

As with HEAvYo (\$2.3.2.1), there is some question as to whether a general version of HTONE/ó exists. In this case, the existence of a constraint demanding $\mathrm{H}$ tone on all syllables would seem to contradict proposals by, e.g., Stevick (1969) and Pulleyblank (1986) that $L$ is the default or unmarked tone in a two-tone system (similarly for $\mathrm{M}$ in a three-tone system). However, when $\mathrm{L}$ is argued to be the default or unmarked tone, this is often done on the grounds that $\mathrm{L}$ tones are phonologically inert. Myers (1998) takes a different approach to the inertness of $L$ tones, arguing that in at least some languages, such as Chichewa, even surface representations contain only $\mathrm{H}$ tones; ' $\mathrm{L}$ ' actually corresponds to the lack of a phonetic target for tone. As Myers notes, if this proposal is to be implemented within OT, constraints banning an actual phonological specification for $\mathrm{L}$ tone must be undominated in the language. Thus, it is not clear that $\mathrm{L}$ is universally less marked than $\mathrm{H}$, perhaps removing one objection to the inclusion of a general HTONE constraint in CON. In any case, as noted in the discussion of HEAVYo (§2.3.2.1), the fundamental claim about $\mathbf{M} / \mathbf{s t r}$ constraints advanced here-that they are subject to the Prominence Condition-does not crucially depend on the existence of general counterparts for each $\mathbf{M} / \mathbf{s t r}$ constraint.

The positional augmentation results concerning tone presented in this dissertation are somewhat preliminary (see $\S 4.2 .3, \S 4.3 .4 .2$ for discussion). One topic for future investigation is the relationship between low tone and positional augmentation effects. The affinity between $\mathrm{H}$ tone and stress is well known; examples are discussed in $\$ 3.2 .1 .2$. But if, as many researchers propose, there is a

prosodic units). 
phonologically relevant prominence hierarchy for (simple) tones ${ }^{29} \mathrm{H}>\mathrm{L}>\mathrm{M}$, there is presumably a universal constraint subhierarchy HTONE $>>$ LTONE $>>$ MTONE. Since low tones are more prominent than mid or zero tones, the constraint LTONE would also pass the Prominence Condition, and we would expect to find L-tone augmentation effects as well. The attraction of $L$ tones to stressed syllables (primary and secondary) is in fact attested; see Kang (1997) on metrical structure and tone in Sukuma and Yip (2000) on tone patterns in Chinese languages. ${ }^{30}$

\subsubsection{HAVESTRESS}

One more positional augmentation constraint, exemplified by the languages discussed in $\S 4.2 .2 .1$, is the constraint HAVESTRESS/Root.

HAVESTRESS/Root

For all syllables $x$, if the head of $x$ is affiliated with a root, then $x$ bears stress ${ }^{31}$

This markedness constraint, which calls for the presence of stress in a root, does qualify as a prominence-enhancing constraint, as predicted by the Prominence Condition. Stress is a local prominence; an element bearing stress is more prominent than adjacent elements (although the ways in which individual languages give phonetic manifestation to phonologically designated stress may differ, incorporating (sub-phonemic) duration, amplitude, pitch-accents, or combinations thereof; Lehiste 1970, Beckman 1986, Hayes 1995).

The question raised during the discussion of HEAVYo (\$2.3.2.1) and HTONE (§2.3.2.5) is relevant here as well: given that $\mathbf{M} / \mathbf{s t r}$ constraints have been treated as relativized versions of general (non-positional) markedness constraints, is there any evidence for a general constraint HAVESTRESS, to which HAVESTRESS/Root is a position-specific counterpart?

HAVESTRESS

For all syllables $x, x$ bears stress

29. Contour tones, particularly HL, are often considered to be even more prominent than H. Another area for future investigation is the relationship between contour tones and positional augmentation effects.

30. de Lacy (1999) also proposes that there is an affinity between $L$ tone and unstressed positions. See Yip (2000) for discussion.

31. See $\S 2.3 .3$ below on the use of prosodic heads to determine affiliations between morphological and prosodic categories. 
With no positional restrictor clause (e.g., 'if the head of $x$ is affiliated with a root, then ...'; \$2.2.2, \$2.3.3) included in the formulation of (43), it becomes a constraint demanding that all syllables bear stress. There is probably no direct evidence in favor of the existence of such a constraint. There appears to be no stress language in which every syllable of every word bears stress (although there are, for example, tone languages in which every syllable bears a surface tone).

As noted above, however, the question of whether all positional augmentation constraints do have general counterparts is a separate problem from the main proposal here, namely, that all $\mathbf{M} / \mathbf{s t r}$ constraints are subject to the Prominence Condition. Furthermore, although there may be no evidence for general HAVESTRESS, the existence of such a constraint does not actually make incorrect predictions about the typology of stress patterns, because of an independent requirement that stress be culminative (Trubetzkoy 1939; Liberman 1975; Hyman 1977; Hayes 1995); in other words, that every prosodic constituent have exactly one head. Where main (word) stress is concerned, this means that every PrWd has exactly one head foot, which has exactly one head syllable, and this syllable bears the main stress of the PrWd.

The requirement that stress be culminative is apparently never violated. ${ }^{32}$ It must in fact be actively enforced, either by a universally undominated constraint or by limits on GEN (the function that generates the set of competing output candidates for each input; Prince \& Smolensky 1993), because when a complex prosodic constituent is composed of two or more elements each of which contributes a toplevel stress, all but one of the stresses is always demoted, if not deleted entirely (as captured in, e.g., the Nuclear Stress Rule of Chomsky \& Halle 1968). For expositional clarity, the following constraint can be used to model the mandatory culminativity of stress (with the understanding that it might actually reflect limits on the form of candidates that GEN can emit rather than being an individual member of the set of constraints). ${ }^{33}$

CULMINATIVITY

Every prosodic constituent has exactly one head

The inclusion of universally undominated CULMINATIVITY in CON (or in GEN), which is independently needed to explain why a form with multiple input stresses

32. Claims have occasionally been made in the literature for words with multiple primary stresses, for example by Voegelin (1935) on Tübatulabal, Dixon (1977) on Yidin ${ }^{y}$, and Woodbury (1987) on Central Alaskan Yupik. However, such cases are controversial; see Hayes 1995 for discussion.

33. The constraint encapsulation CULMINATIVITY invoked here is based on that in Alderete (1999b), except that Alderete considers CULMINATIVITY to be potentially violable. 
will always surface with exactly one output stress, can also account for why no language has stress on every syllable, even if general HAVESTRESS is in fact part of CON.

One final point about HaveStress is that, while all of the HAVESTRESS constraints considered in $\$ 4.2$ are for main stress, presumably there are HAVESTRESS constraints corresponding to stress at all relevant levels of the prosodic hierarchy, in particular, foot-level (secondary) stress. Finding evidence for general and positional HAVESTRESS constraints at the foot level is a topic left for future investigation.

\subsubsection{Summary}

This section (§2.3.2) has examined a number of markedness constraints that have attested $\mathbf{M} / \mathbf{s t r}$ counterparts (see chapters 3 and 4). Each of these constraints has been shown to be an augmentation constraint, a constraint that calls for some perceptually prominent characteristic. Thus, the Prominence Condition, a constraint filter that passes $\mathbf{M} / \mathbf{s t r}$ constraints only when they are built from augmentation constraints, makes correct predictions about which markedness constraints can be relativized to strong positions.

Another objective of this section has been to give each augmentation constraint an explicit formulation that unambiguously identifies the constraint focus. As explained in $\$ 2.2 .2$ above, it is the nature of the focus that determines the strong positions to which a given constraint can be relativized. The following section (§2.3.3) now examines what happens when the augmentation constraints presented above are combined with the members of the set of strong positions. Many of the resulting positional constraints are legitimate constraints. Others, however, are meaningless because they involve a "domain mismatch," that is, a strong position that, because of its size or character, is not compatible with the focus of the constraint in question.

\subsubsection{Augmentation constraints and strong positions}

This section examines the predictions that are made when the $\mathbf{C} /$ str constraint schema, which forms position-specific constraints ( $\$ 2.2 .2)$, is applied to the strong positions from $\S 1.3 .1$ and the augmentation constraints from $\$ 2.3 .2$. All of the $\mathbf{M} / \mathbf{s t r}$ constraints thus formed are legitimate constraints according to the Prominence Condition, because they are relativized versions of augmentation constraints. However, not all of these constraints are meaningful, because in some 
cases the constraint focus, which plays a crucial role in determining the formulation of any relativized version of the constraint, is not compatible with a particular strong position. This kind of incompatibility is termed a domain mismatch (§2.2.2).

First, the five strong positions stressed syllable, long vowel, onset, initial syllable, and root are explicitly characterized, so that their semantic contribution to the positional restrictor if/then clauses introduced by the $\mathrm{C} /$ str schema can be specified. Then, a chart is presented that shows all the possible combinations of the augmentation constraints from $\S 2.3 .2$ with these strong positions. Among the positional augmentation constraints thus generated, meaningful constraints are distinguished from domain mismatches. The chart also indicates whether or not the expected M/str constraints are attested (and if so, where in chapters 3 and 4 they are discussed). Several of the expected $\mathbf{M} / \mathbf{s t r}$ constraints for the psycholinguistically strong positions, initial syllable and root, are conspicuously unattested, but these are the $\mathbf{M} / \Psi$ str constraints that the Segmental Contrast Condition, the constraint filter discussed in $\$ 2.4$ below, predicts not to be included in CON.

The $\mathrm{C} /$ str schema, introduced in $\S 2.2 .2$, is repeated in (45). This is the constraint schema that constructs all relativized constraints, including $\mathbf{M} / \mathbf{s t r}$ constraints.

The C/str schema

C/str For all $y$, if $y$ is a str, then $\mathbf{C}$ holds of $y$

where $y$ is an element in the focus of the constraint $\mathbf{C}$

As described above, the $\mathrm{C} /$ str schema has been given this particular characterization so that the formulation of any relativized constraint is predictable from the formulation of the general constraint $\mathbf{C}$ and the nature of the strong position str. Formulations of the general versions of the augmentation constraints under investigation here have been presented in \$2.3.2. In (46) and the discussion that follows, explicit characterizations are now given to the strong positions, so that their contribution to the formulation of a given $\mathbf{M} / \mathbf{s t r}$ constraint can also be made explicit. (See $\$ 2.4 .2$ for further discussion of the division between phonetically and psycholinguistically prominent positions, introduced in $§ 1.3 .1$.) 
(46) Strong positions as positional restrictor clauses

(a) Phonetically strong positions

\begin{tabular}{|l|l|l|}
\hline Position & Restrictor clause & $\begin{array}{l}\text { Compatible } \\
\text { constraint foci }\end{array}$ \\
\hline$\sigma$ & $\ldots$ if $x$ is a ó, then ... & syllable \\
\hline $\mathrm{V}:$ & $\ldots$ if $x$ is a $\mathrm{V}:$, then $\ldots$ & segment \\
\hline Onset & $\ldots$ if $x$ is $\mathrm{C}_{[+ \text {release] }}$, then $\ldots$ & segment \\
\hline
\end{tabular}

(b) Psycholinguistically strong positions

\begin{tabular}{|l|l|l|}
\hline Position & Restrictor clause & $\begin{array}{l}\text { Compatible } \\
\text { constraint foci }\end{array}$ \\
\hline$\sigma_{1}$ & $\begin{array}{c}\ldots \text { if } x \text { is the leftmost } \sigma \text { whose } \\
\text { head is affiliated with MWd } \\
m, \text { then } \ldots\end{array}$ & syllable \\
\hline Root & $\begin{array}{c}\ldots \text { if }[\text { the head of] } x \text { is affiliated } \\
\text { with a root, then } \ldots\end{array}$ & $\begin{array}{l}\text { Any focus is } \\
\text { compatible }\end{array}$ \\
\hline
\end{tabular}

The formalization of the phonetically strong positions (46a) as positional restrictor clauses is fairly straightforward. Stressed syllable (ó) is intended to mean "head syllable of the head foot of the prosodic word," that is, the syllable that bears main stress. ${ }^{34}$ Long vowel (V:) is intended to mean "vowel associated with more than one mora." Finally, as noted in $\$ 1.3 .1$, the phonetically strong position abbreviated as onset for the sake of notational convenience is more accurately identified as "released consonant" (Kingston 1985, 1990; Lombardi 1991, 1999). Given these characterizations, constraints that can be meaningfully relativized to these positions are those whose focus refers to a syllable, a segment, and a segment respectively, as indicated in (46a).

34. As noted in $§ 1.3 .1$, footnote 7, other prosodic heads-including the head syllables of non-head feet ( $(\mathbf{\sigma})$ and phrasal heads-are probably also to be included in the set of strong positions. However, in the present discussion, attention is restricted to main-stress syllables. 
The formalization of the psycholinguistically strong positions (46b) requires somewhat more discussion. The complicating factor here is that both of these positions are morphologically defined; the root obviously so, and the initial syllable because it is the initial syllable of the morphological word (MWd) that is the relevant strong position (\$4.4). Because morphological structure and prosodic structure are on different "planes" of phonological representation, these two kinds of structure can be directly related only with reference to the segments that they share (as noted in various discussions of morphological-prosodic alignment constraints, especially McCarthy \& Prince 1993a:89; see also Kager 1999:11). Thus, when these morphologically defined positions need to be related to prosodic constituents, this can be done by way of the segments that serve as heads of those prosodic constituents (see also McCarthy 2000ab on faithfulness to prosodic heads through segmental correspondence). The specific examples that follow help clarify this proposal.

First, in accordance with the use of prosodic heads to mediate between prosodic and morphological structure, the initial syllable must be defined in such a way as to relate the syllable and MWd aspects of this position through the (terminal) head of the syllable, since this is a segment, and only segments can have a morphological affiliation. Thus, the initial syllable of MWd $m$ is identified in (46b) as the leftmost syllable whose head is morphologically affiliated with $\mathrm{m}$. Constraints that can be meaningfully relativized to this position are, as shown in (46b), those whose focus refers to a syllable.

The other psycholinguistically strong position, the root, is different from all other strong positions, including the initial syllable, in that it is defined entirely by morphological affiliation, with no additional reference to phonological elements of any size. Therefore, no $\mathbf{M} / \mathbf{R o o t}$ constraint is predicted to result in a domain mismatch, regardless of the nature of the focus of $\mathbf{M}$. However, if a constraint whose focus is a prosodic constituent (such as HAVESTRESS (43), for which the focus is a syllable) is to be relativized to the strong position root, this also must be done through reference to the segment that is the (terminal) head of the prosodic constituent in question. In such a case, the phrase 'the head of is automatically included in the positional restrictor clause for roots, as indicated with brackets in $(46 b){ }^{35}$

35. Because an $\mathbf{M} / \mathbf{R o o t}$ constraint with a prosodic category as its focus must be relativized in this way-through reference to the head of the syllable-a prediction is made that a syllable with an epenthetic vowel for its head, even if it contains other root-affiliated segments, will not be subject to the M/Root constraint. For example, HAVESTRESS/Root ('for all syllables $x$, if $x$ is affiliated with a root, then $x$ bears stress') is predicted not to be sensitive 
Given the constraint formulations in $§ 2.3 .2$ above and the characterizations of the positional restrictor clauses in (46), it is possible to predict which constraints can be relativized to which positions without giving rise to a domain mismatch. The chart in (47) shows all possible combinations of the constraints and positions under consideration. For each constraint, the types of elements referred to in the constraint's focus are indicated with the notation - foc. $\mathbf{M} / \mathbf{s t r}$ combinations that involve a domain mismatch are identified with shaded cells and the notation $\boldsymbol{D M}$. For each non-mismatch, if the constraint is attested in the language examples discussed in chapters 3 and 4 , the corresponding cell is marked with $\checkmark$ and a crossreference to the relevant examples is given. If the constraint is not attested, the cell is marked with one of three codes (SCC, vac [uous], or note) and the constraint is given further discussion below the chart.

to epenthetic vowels in roots. This prediction appears to be borne out in Chukchee (Krause 1979), where roots preferentially bear stress unless the only vowels they contain are epenthetic schwas, and there is no general ban on stressed schwa. 
(47) Predicted positional augmentation constraints

\begin{tabular}{|c|c|c|c|c|c|}
\hline & \multicolumn{3}{|c|}{ (a) $\mathbf{M} / \Phi_{\text {str }}$} & \multicolumn{2}{|c|}{ (b) $M / \Psi_{\text {str }}$} \\
\hline & ó & V: & onset & $\sigma_{1}$ & root \\
\hline $\begin{array}{l}\text { HEAVYo } \\
\text { - foc: } \sigma\end{array}$ & $\begin{array}{c}\checkmark \\
\$ 3.2 .1 .1\end{array}$ & $D M$ & $D M$ & $(S C C)$ & $(S C C)$ \\
\hline $\begin{array}{l}\text { *PEAK/X } \\
\text { - foc: } s e g, \sigma\end{array}$ & $\begin{array}{c}\checkmark \\
\$ 3.2 .1 .3\end{array}$ & $\begin{array}{c}\checkmark \\
\$ 3.3\end{array}$ & (note) & $(S C C)$ & $(S C C)$ \\
\hline $\begin{array}{l}\text { ONSET } \\
\cdot f o c: \sigma\end{array}$ & $\begin{array}{c}\checkmark \\
\S 3.2 .2\end{array}$ & $D M$ & DM & $\begin{array}{c}\checkmark \\
\$ 4.2 .1 .1\end{array}$ & $(S C C)$ \\
\hline $\begin{array}{l}{ }^{*} \text { ONSET/X } \\
\text { - foc: } \mathrm{seg}, \sigma\end{array}$ & $\underset{\S}{\S 3.2 .2}$ & $\begin{array}{c}(\checkmark) \\
(v a c .)\end{array}$ & (note) & $\begin{array}{c}\checkmark \\
\S 4.2 .1 .2\end{array}$ & $(S C C)$ \\
\hline $\begin{array}{l}\text { HAVECPLACE } \\
\text { - foc: C }\end{array}$ & $D M$ & $D M$ & $\underset{\checkmark}{\$ 3.4}$ & $D M$ & $(S C C)$ \\
\hline $\begin{array}{l}\text { HTONE } \\
\text { • foc: } \sigma\end{array}$ & $\begin{array}{c}\checkmark \\
\S 3.2 .1 .2\end{array}$ & $D M$ & $D M$ & $\begin{array}{c}(S C C ?) \\
(\$ 4.2 .1 .3)\end{array}$ & $\begin{array}{c}(S C C ?) \\
(\$ 4.2 .2 .3)\end{array}$ \\
\hline $\begin{array}{l}\text { HAVESTRESS } \\
\text { - foc: } \sigma\end{array}$ & $\begin{array}{c}(\Omega) \\
(\text { vac. })\end{array}$ & $D M$ & $D M$ & $\begin{array}{c}\text { (note) } \\
(\$ 4.2 .1 .3)\end{array}$ & $\begin{array}{c}\checkmark \\
\$ 4.2 .2 .1\end{array}$ \\
\hline
\end{tabular}

The special cases indicated in the chart in (47) are as follows. A number of

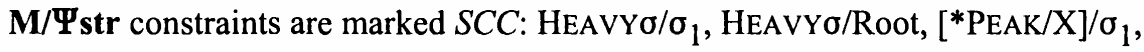
[ ${ }^{*}$ PEAK/X]/Root, ONSET/Root, $\left[{ }^{*}\right.$ ONSET/X]/Root, and HAVECPLACE/Root. These are all well-formed constraints by the $\mathrm{C} /$ str schema, and they pass the Prominence Condition. Nevertheless, they are excluded from CON because they fail to pass the Segmental Contrast Condition, a constraint filter on $\mathbf{M} / \Psi$ str constraints that is discussed in $\$ 2.4$ below and in $\S 4.3$. (HTONE/ $\sigma_{1}$ and HTONE/Root may also be blocked by the Segmental Contrast Condition; see $\S 4.3 .4 .2$ for discussion.)

Two of the M/str constraints in (47) are marked vacuous: [ ${ }^{*}$ ONSET/X]/V: and HAVESTRESS/o. These constraints would have the formulations in (48). 
(48) (a) $\left[{ }^{*} \mathrm{ONSET} / \mathrm{X}\right] / \mathrm{V}:$ For every segment $a$ that is the leftmost pre-moraic segment of some syllable $x$, if $a$ is a $\mathrm{V}$ :, then $|a|<\mathrm{X}$

where $|y|$ is the sonority of segment $y$

$\mathrm{X}$ is a particular step on the segmental sonority scale

(b) HAVESTRESS/ $\sigma$ For all syllables $x$, if $x$ is a ó , then a $x$ bears stress

As can be seen in (48), these two constraints are not technically domain mismatches, because there is no formal incompatibility between the focus of $\mathbf{M}$ and the restrictor clause introduced by the strong position. However, they are like domain mismatches in that they are tautological-they are vacuously satisfied by every possible candidate. [ $\left.{ }^{*} \mathrm{ONSET} / \mathrm{X}\right] / \mathrm{V}:$ is always satisfied because no segment can simultaneously be non-moraic and bimoraic. HAVESTRESS/ó is always satisfied because it simply demands of a stressed syllable that it be a stressed syllable. Since these two constraints are tautological, they can play no active role in the phonologies of individual languages (and may even be ruled out by a constraint filter against tautological constraints; see the remarks on domain-mismatch cases in $\S 2.2 .2$ ).

Finally, there are three constraints in (47) marked note: HAVESTRESS $/ \sigma_{1}$, $\left[{ }^{*} \mathrm{ONSET} / \mathrm{X}\right] / \mathrm{Ons}$, and $\left[{ }^{*} \mathrm{PEAK} / \mathrm{X}\right] / \mathrm{Ons}$. These constraints are predicted by the model to exist, but (at this time) it is not clear that there is evidence of their activity in the phonology of any language. These constraints are predicted to exist because they are well-formed according to the $\mathrm{C} /$ str schema, they pass the Prominence Condition, they pass the Segmental Contrast Condition (see $\$ 2.4$ below), and, as shown in (49), they do not involve a domain mismatch.

(a) HAVESTRESS $/ \sigma_{1}$

For all syllables $x$, if $x$ is the leftmost $\sigma$ whose head is affiliated with MWd $m$, then $x$ bears stress 
(b) $\left[{ }^{*} \mathrm{ONSET} / \mathrm{X}\right] / \mathrm{Ons}$

where

(c) $\left[{ }^{*} \mathrm{PEAK} / \mathrm{X}\right] /$ Ons
For every segment $a$ that is the leftmost premoraic segment of some syllable $x$, if $a$ is $\mathrm{C}_{[+ \text {release }]}$, then $|a|<\mathrm{X}$

$|y|$ is the sonority of segment $y$

$\mathrm{X}$ is a particular step on the segmental sonority scale

For every segment $a$ that is the head of some syllable $x$, if $a$ is $\mathrm{C}_{[+ \text {release] }}$, then $|a|>\mathrm{X}$

where $|y|$ is the sonority of segment $y$

$\mathrm{X}$ is a particular step on the segmental sonority scale

HAVESTRESS/ $\sigma_{1}(49 a)$ is a constraint that requires the initial syllable to bear stress. As noted in $\$ 4.2 .1 .3$, there are indeed languages with mandatory initial stress; the problem is only that it may be impossible to distinguish HAVESTRESS $/ \sigma_{1}$ empirically from ALIGN-L constraints on stress.

[*ONSET/X]/Ons (49b) is a constraint subhierarchy whose members demand that a syllable "onset" (formally, the leftmost pre-moraic segment) be low in sonority if it is an instance of the strong position "onset" (formally, a released consonant). The leftmost pre-moraic segment of a syllable will nearly always be a released consonant. As a result, in most languages, $\left[{ }^{*} \mathrm{ONSET} / \mathrm{X}\right] / \mathrm{Ons}$ will assign exactly the same violation marks as general ${ }^{*}$ ONSET/X. Only in a language where the leftmost pre-moraic segment is not always released (as might be the case in an obstruent-obstruent onset cluster, as argued for example by Steriade 1997) can the effects of [ $\left.{ }^{*} \mathrm{ONSET} / \mathrm{X}\right] / \mathrm{Ons}$ and general ${ }^{*} \mathrm{ONSET} / \mathrm{X}$ be distinguished; such a case is not included in the languages considered in chapters 3 and 4 .

$\left[{ }^{*} \mathrm{PEAK} / \mathrm{X}\right] / \mathrm{Ons}(49 \mathrm{c})$ is a constraint subhierarchy whose members demand that if a syllable peak consists of a released consonant, that peak must be high in sonority. Again, the effects of this constraint could be distinguished from those of general $* \mathrm{PEAK} / \mathrm{X}$ only in a language that makes a distinction between consonantal syllable peaks that are released and consonantal syllable peaks that are not released, and a relevant situation has not been found among the languages considered here.

Thus, the three constraints marked note in (47) are constraints that are predicted by the model to exist, but either overlap to a great extent with other attested constraints, or would potentially be active in only a very limited set of circumstances; it is not surprising that empirical evidence for these constraints has yet to be found. 
The rest of the formally possible $\mathbf{M} / \mathbf{s t r}$ constraints in (47), marked with $\checkmark$, are empirically attested, just as the model predicts. Languages in which these $\mathbf{M} / \mathbf{s t r}$ constraints play an active role are discussed in the sections of chapters 3 and 4 that are indicated in the corresponding cells in the chart.

\subsubsection{Summary}

This section has implemented the first proposed restriction on $\mathbf{M} / \mathbf{s t r}$ constraints, the Prominence Condition, as a filter in the Schema/Filter model of CON. The Prominence Condition accepts an $\mathbf{M} / \mathbf{s t r}$ constraint only if the corresponding general $\mathbf{M}$ constraint is an augmentation constraint (calling for the presence of perceptually prominent characteristics). This filter has been shown to make correct predictions about which $\mathbf{M}$ constraints are seen to have $\mathbf{M} / \mathbf{s t r}$ counterparts in the languages discussed in chapters 3 and 4, because all the general $\mathbf{M}$ constraints from which the attested $\mathbf{M} / \mathbf{s t r}$ constraints are built are indeed augmentation constraints. Finally, all the possible combinations of these augmentation constraints with the members of the set of strong positions have been enumerated, and the inventory of $\mathbf{M} / \mathbf{s t r}$ constraints predicted by the model has been shown to match well with the inventory of attested M/str constraints - once the effects of the Segmental Contrast Condition, the constraint filter that is the topic of the following section, have also been taken into account.

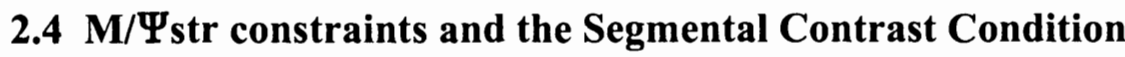

Up to this point, the discussion has focused on general properties of augmentation constraints and strong positions, and thus on the predictions that the model makes about $\mathbf{M} / \mathbf{s t r}$ constraints in general. However, not all strong positions obtain their privileged status from the same kinds of substantive considerations. As originally proposed by Beckman $(1997,1998)$ and Casali $(1996,1997)$, there are two classes of strong positions. Phonetically strong positions, abbreviated $\Phi_{s t r}$, are those positions that have particularly salient phonetic cues to the recovery of phonological contrasts; examples discussed here are the stressed syllable, the long vowel, and the onset (released consonant). Psycholinguistically strong positions, abbreviated $\Psi_{\text {str }}$, are positions that are important in speech processing; specifically, it is proposed here that psycholinguistically strong positions are those positions that play an important role in the early stages of word recognition (see $\$ 2.4 .2$ below and $\$ 4.3 .1$ 
on this distinction). Thus, psycholinguistically strong positions include the initial syllable and the morphological root, but, crucially, not the stressed syllable.

Because only psycholinguistically strong positions are directly involved in early-stage word recognition ( $\$ 4.3$ ), there are substantive pressures on these strong positions that are not relevant for phonetically strong positions. One such pressure is the drive to avoid the neutralization of phonological contrasts specifically in these positions, since this would make word recognition less efficient without reducing the overall complexity of the language's inventory of contrastive categories ( $\$ 4.3 .5)$. Another substantive pressure relevant for psycholinguistically strong positions is the drive to facilitate what is known as segmentation of the speech stream, that is, division of the incoming acoustic signal into words so that word recognition can be accomplished (see $\S 4.3 .4 .1 .3$ on the difficulties of word segmentation in speech perception).

These two pressures are formalized here as a constraint filter, the Segmental Contrast Condition, which evaluates $\mathbf{M} / \Psi$ str constraints-augmentation constraints relativized to psycholinguistically strong positions. The Segmental Contrast Condition blocks $\mathbf{M} / \Psi$ str constraints from inclusion in CON if their satisfaction would neutralize contrasts that are important in early-stage word recognition (i.e., "segmental" contrasts, as distinct from "prosodic" contrasts, but see $\S 4.3 .4 .2$ on the possibility that tone patterns with segmental contrasts), unless the property required by the constraint is one that aids in left-edge demarcation of words.

With the Segmental Contrast Condition in place, the model predicts that certain $\mathbf{M} / \Psi$ str constraints that pass the Prominence Condition (\$2.3.1), and do not involve any domain mismatch between the constraint focus and the strong position (\$2.3.3), will nevertheless fail to be part of CON if they call for the neutralization of psycholinguistically relevant contrasts. The differences between the columns for stressed syllable (a phonetically strong position) and initial syllable (a psycholinguistically strong position) in (47) above demonstrate the accuracy of this prediction. The two strong positions are both syllable-sized, so they are formally compatible with the same set of augmentation constraints (as far as the $\mathrm{C} /$ str schema and domain-mismatch avoidance are concerned). Nevertheless, there are more $\mathbf{M} / \boldsymbol{\sigma}$ constraints than $\mathbf{M} / \sigma_{1}$ constraints attested. Likewise, the absence of feature-related M/Root constraints in (47) matches the predictions made by the Segmental Contrast Condition.

This section presents the Segmental Contrast Condition and discusses its implications for the theory of positional augmentation constraints. (More detailed discussion of the nature of the Segmental Contrast Condition, and a review of the psycholinguistic evidence that supports the characterization of this constraint filter as it is given here, are provided in $\S 4.3$; cross-references to relevant subsections of $\S 4.3$ are given throughout this section.) $\S 2.4$.1 presents a characterization of the 
Segmental Contrast Condition as a constraint filter, describes how constraints can be tested for compliance with this filter, and identifies the $\mathbf{M} / \Psi$ str constraints that pass the filter. \$2.4.2 gives an overview of the difference between phonetically and psycholinguistically strong positions that is fundamental to the Segmental Contrast Condition (this difference is discussed in greater detail in $\S 4.3$ ). A preliminary proposal is also presented concerning another domain of phonology that can be fruitfully analyzed in terms of a constraint filter distinguishing between phonetically and psycholinguistically strong positions: the close relationship between neutralization avoidance in phonetically strong positions and the specific phonetic characteristics of those positions ("licensing by cue"), which has been investigated especially in the work of Steriade (1993, 1995, 1997, 1999ab).

\subsubsection{The Segmental Contrast Condition}

The Segmental Contrast Condition is a constraint filter on $\mathbf{M} / \Psi$ str constraints that models the substantive requirements on psycholinguistically strong positions described above: the pressure to avoid the neutralization of contrasts in these psycholinguistically important positions, and the pressure to facilitate segmentation of the speech stream by demarcating the left edges of words. Because these two substantive pressures interact, they are modeled as a single, two-part constraint filter.

\section{Segmental Contrast Condition}

If a constraint is of the form $\mathbf{M} / \Psi$ str, then it must meet one of the following two conditions:

I. Satisfaction of the $\mathbf{M}$ constraint from which the $\mathbf{M} / \mathbf{\Psi}$ str constraint is built does not alter features that are distinguished in early-stage word recognition.

or

II. Tstr is $\sigma_{1}$, and satisfaction of the $M / \Psi$ str constraint serves to demarcate the left edge of $\sigma_{1}$.

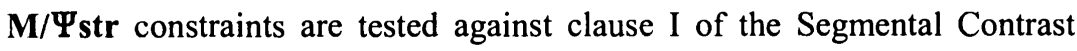
Condition as follows. From an arbitrary input, two candidates are generated that are "minimally different" with respect to their faithfulness violations (as defined for the 
Prominence Condition in $\S 2.3 .1$ above), such that one satisfies the general version of $\mathbf{M}$ but the other violates it. If the two candidates, while being perceived, cause the same pattern of lexical activation-that is, cause the same set of lexical entries to be activated to the same extent - then the contrast neutralized by $\mathbf{M}$ is irrelevant for early-stage word recognition, and the $\mathbf{M} / \Psi$ str constraint passes clause I of this filter.

Constraints are tested against clause II of the Segmental Contrast Condition in a similar manner. Again, from an arbitrary input two minimally different candidates are generated such that one satisfies $\mathbf{M} / \mathbf{\Psi}$ str (the positional version of the constraint this time, since it is a particular position, namely initial syllable, to which clause II is sensitive) and the other violates it. If $\Psi$ str is something other than initial syllable, then $\mathbf{M} / \Psi$ str fails clause II immediately. If $\Psi$ str is initial syllable, and the leftmost segments of the two candidates differ, then the M/ Tstr constraint passes clause II. ${ }^{36}$

Like the Prominence Condition, discussed in $\$ 2.3$ (and like the Inductive Grounding Principle of Hayes 1999a), the Segmental Contrast Condition is a filter that evaluates formally possible constraints by using extra-phonological information. In this case, it is information from the processing and word-recognition system that determines which potential constraints should be included in CON. It should be noted that the Prominence Condition and the Segmental Contrast Condition are not ordered or ranked in any way, as is true of constraint filters in general (i.e., constraint filters are not like the constraints of an OT grammar). Any constraint that fails any of the filters is simply not included in CoN.

The Segmental Contrast Condition makes explicit predictions about what kinds of augmentation constraints can have $\mathbf{M} / \Psi$ str counterparts. Namely, $\mathbf{M} / \Psi$ str constraints are not permitted to manipulate phonological contrasts that are involved in early-stage word recognition, with the exception of onset manipulation in initial syllables, which is allowed by clause II. Since stress, even though it can be phonologically contrastive, is not crucially involved in this aspect of word recognition (\$4.3.4), augmentation constraints that manipulate stress in psycholinguistically strong positions are predicted to exist. In fact, there is empirical

36. Clause II of the Segmental Contrast Condition is formulated to be as general as possible: an $\mathbf{M} / \Psi$ str constraint passes clause II if its satisfaction affects the left edge of $\sigma_{1}$, with no further specification as to how the left edge must be affected. As a result, constraints that manipulate the left edge of $\sigma_{1}$ in ways unrelated to prominence enhancement will pass clause II just as the legitimate, empirically attested ONSET $/ \sigma_{1}$ and $\left[{ }^{*} \mathrm{ONSET} / \mathrm{X}\right] / \sigma_{1}$ will. However, any left-edge-effecting constraint that is not related to prominence enhancement will fail to pass the Prominence Condition (\$2.4.1) and will be excluded from CoN for that reason. 
evidence in support of the existence of HAVESTRESS/Root (\$4.2.2.1), and evidence that is compatible with the existence of HAVESTRESS/ $\sigma_{1}(\$ 4.2 .1 .3)$.

It is necessary also to consider the implications of the Segmental Contrast Condition for positional augmentation constraints that manipulate tone, such as HTONE $/ \sigma_{1}$ and HTONE/Root. If tone is lexically contrastive in a given language, then it is possible to have two lexical entries that are phonologically distinguished only by tone. However, psycholinguistic results reported to date are not conclusive about whether tone is like segmental contrasts, in that it distinguishes lexical entries during early-stage word recognition, or whether tone is like stress, in that it does not. Until the role of tone in word recognition is better understood, it is not clear whether HTONE $/ \sigma_{1}$ and HTONE/Root pass the Segmental Contrast Condition. See $\S 4.3 .4 .2$ for discussion.

On the other hand, augmentation constraints that manipulate segmental contrasts, namely, HEAVYo, *PEAK/X, ONSET, *ONSET/X, and HAVECPLACE, all fail clause I of the Segmental Contrast Condition because their satisfaction does affect segmental featural contrasts. Therefore, these constraints do not have M/Root counterparts at all, and only ONSET and *ONSET/X have $\mathbf{M} / \sigma_{1}$ counterparts, because these are the only $M / \sigma_{1}$ constraints that are relevant for the left edge of the initial syllable $^{37}$ and therefore pass clause II of the filter.

In summary, there is a tension inherent in the substantive considerations related to $\mathbf{M} / \Psi$ str constraints. On the one hand, psycholinguistically strong positions, like all strong positions, would be perceptually enhanced by the satisfaction of augmentation constraints relativized to these positions. On the other hand, when an augmentation constraint is satisfied, some potential phonological contrast is neutralized - the perceptually salient characteristic mandated by the constraint is always present, so the presence versus absence of that characteristic can no longer serve as the basis for a contrast-and the neutralization of segmental contrasts specifically in psycholinguistically strong positions is detrimental to efficient word recognition. The inclusion of the Segmental Contrast Condition in the Schema/Filter model of CON protects psycholinguistically strong positions from segmentalcontrast neutralization, including neutralization for purposes of augmentation, except where augmentation would help in left-edge demarcation for segmentation of the speech stream.

37. HAVECPLACE $/ \sigma_{1}$ might look like another left-edge-related constraint, but since the focus of HAVECPLACE is a segment, relativizing this constraint to the initial syllable leads to a domain mismatch (47). 


\subsubsection{Classifying strong positions}

The Segmental Contrast Condition, presented in the preceding section, is a constraint filter that captures certain substantive requirements holding of markedness constraints relativized to psycholinguistically strong positions. These substantive requirements have their basis in the special status of psycholinguistically strong positions in early-stage word recognition. In this section, the distinction between psycholinguistically and phonetically strong positions is discussed, and the proposal that only initial syllable and root qualify as psycholinguistically strong positions is defended (see also the more detailed discussion in \$4.3.3). Consideration is also given to another phonological phenomenon that distinguishes phonetically and psycholinguistically strong positions: "licensing by cue" effects (Steriade 1993, 1995, 1997, 1999ab) in resistance to positional neutralization.

\subsubsection{Psycholinguistically strong positions}

The definition of a psycholinguistically strong position given here is based on the proposals of Beckman $(1997,1998)$ and Casali $(1996,1997)$, who recognize the phonologically privileged status of these positions in their ability to resist positional neutralization. These researchers state (emphasis added),

Positions which are psycholinguistically prominent are those which bear the heaviest burden of lexical storage, lexical access and retrieval, and processing: root-initial syllables, roots and, to some degree, final syllables. (Beckman 1998:1)

... preferential preservation of word-initial material may be related to the crucial function initial segments play in speech processing ... (Casali 1997:494)

However, the proposal made here is more specific than Beckman's and Casali's proposals: it is the importance of a particular position specifically for early-stage word recognition, ${ }^{38}$ not just for speech processing in general, that gives it the status of a psycholinguistically strong position. Thus, initial syllables and roots qualify as psycholinguistically strong positions, but stressed syllables do not, even though they are clearly not irrelevant for speech processing (\$4.3.4.1). Crucially, the involvement of stressed syllables in speech processing is different and less direct.

38. The term early-stage word recognition is used here to indicate the stage of speech processing in which a set of lexical entries that are similar to the incoming acoustic signal is initially activated (see $§ 4.3 .1$ ). 
Stress patterns are used in later stages of word recognition to find the best match between the incoming signal and one of the lexical items that has already been activated in the early stage, and stressed syllables are used in some languages to help with segmentation of the speech stream during processing. However, earlystage word recognition is not carried out with direct reference to stressed syllables. Justification for this claim is provided throughout $\S 4.3$, most explicitly in $\S 4.3 .4$.

\subsubsection{Phonetically strong positions}

The theories of positional faithfulness developed by Beckman $(1997,1998)$ and Casali (1996, 1997), following work by, e.g., Steriade (1993, 1995, 1997) and Jun (1995), also recognize certain positions that have a special ability to license contrasts, or resist neutralization processes, because they are positions that have intrinsic phonetic salience. These positions include the stressed syllable, the long vowel, and the syllable onset (released consonant).

As discussed extensively by Steriade (1993, 1995, 1997, 1999ab; see also, e.g., Kingston 1985, 1990 and Lombardi 1991, 1999), there is a close relationship between the kind of salience that a phonetically strong position has and the kinds of contrasts that it has a special ability to license. (Preliminary discussion of how this relationship could itself be modeled in the Schema/Filter theory of CON is found in $\$ 2.4 .2 .3$ below.) For example, onset consonants are characteristically released into a following sonorant, so they are better able than characteristically unreleased consonants (such as syllable codas) to license contrasts for which auditory cues are found in the consonantal release-e.g., voicing, aspiration, or many place features. However, syllable onsets (and released consonants in general) show no special licensing ability for retroflexion, because, Steriade (1993 et seq.) argues, the most salient perceptual cues for retroflexion are found in the VC transition, not in the consonantal release.

While the relationship between phonetically strong positions and featural licensing abilities (i.e., possible positional faithfulness constraints) is highly restricted, however, the relationship between these positions and augmentation constraints is remarkably free. As observed in the chart in (47) in \$2.3.3, phonetically strong positions are eligible for any kind of positional augmentation constraint that can legitimately be relativized to a position of that particular size. Most strikingly, a phonetically strong position can undergo augmentation processes that add prominent properties completely unrelated to the properties that make that 
particular position a salient one. ${ }^{39}$ For example, the position stressed syllable is prominent for phonetic reasons related to properties of vowels (or rimes), such as increased duration or amplitude relative to unstressed syllables. Nevertheless, stressed syllables undergo augmentation processes involving consonantal features, i.e., ONSET/ó and [*ONSET/X]/ó.

Thus, it can be concluded that there are no restrictions on positional augmentation constraints for phonetically strong positions, other than the basic Prominence Condition, which applies to all $\mathbf{M} / \mathbf{s t r}$ constraints. In terms of the Schema/Filter model of CON, this pattern supports the proposal that there are no filters that apply specifically to $\mathbf{M} / \boldsymbol{\Phi}_{\text {str }}$ constraints. Furthermore, the Segmental Contrast Condition is predicted not to apply to these positions, since they are not directly relevant in early-stage word recognition. This prediction is met, since

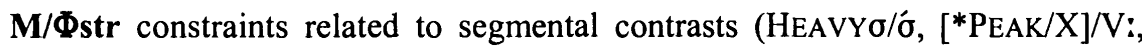
$\left[{ }^{*} \mathrm{PEAK} / \mathrm{X}\right] / \sigma, \mathrm{onSET} / \sigma, \boldsymbol{\sigma}\left[{ }^{*} \mathrm{ONSET} / \mathrm{X}\right] / \sigma, \mathrm{o}$ HAVECPLACE/Onset) are well attested.

\subsubsection{Excursus: "Licensing by Cue" as a filter on $F / \Phi_{\text {str }}$ constraints}

Given that there is a formal distinction between phonetically and psycholinguistically strong positions that is relevant for one constraint filter, the Segmental Contrast Condition, it is to be expected that other constraint filters might also make use of this distinction. One plausible case has to do with the way that positional faithfulness effects-the special resistance of material in strong positions to featural neutralization-seem to differ between phonetically and psycholinguistically strong positions. When positional faithfulness constraints ${ }^{40}$ are

39. As noted in Chapter 1, this is evidence for the status 'phonetically strong position' as an abstract, formal designation that is distinct from the phonetic properties that allow a position to qualify as strong.

40. As discussed in $§ 5.2$, several formally distinct proposals have been made for analyzing positional neutralization effects: $\mathbf{F} / \mathbf{s t r}$ constraints, $\mathbf{M} / \mathbf{w k}$ constraints, and $\operatorname{CoINCIDE}(\alpha, \mathbf{s t r})$ constraints. $\mathbf{M} / \mathbf{w k}$ constraints are not considered here because there are problems inherent in adopting constraints that refer directly to weak positions; see $§ 1.3 .2$. F/str and COINCIDE constraints, while formally different in how they assess violations and in how they are used to account for positional neutralization effects, both refer only to strong positions. Since the discussion in this section shows that there are substantive restrictions on $\mathbf{F} / \Phi_{\text {str }}$ constraints in a system that accounts for positional neutralization with $\mathbf{F} / \mathbf{s t r}$ constraints, this would obviously translate into substantive restrictions on $\operatorname{COINCIDE}(\alpha, \Phi$ str) constraints if the other approach to positional neutralization is taken. In either case, the same substantive restriction must be captured in a filter on the relevant $\Phi_{s t r}$-related constraints. 
high ranking, they allow strong positions to "license features," that is, to resist neutralization processes that affect other positions. Therefore, an examination of the contrasts that are permitted only in a particular strong position gives an indication of the kinds of positional faithfulness constraints that exist for that position.

For positional augmentation constraints, as just seen, there is a filter specific to $\mathbf{M} / \Psi$ str constraints that restricts the possibilities for augmentation in psycholinguistically strong positions. For positional faithfulness constraints, the reverse pattern is observed: the phonetically strong positions are particularly restricted in their ability to form $\mathbf{F} / \boldsymbol{\Phi}$ str constraints, whereas no such restriction is found for $\mathbf{F} / \Psi$ str constraints. Although a detailed investigation of positional faithfulness effects sorted by type of position is not possible here, this section presents a preliminary proposal for how restrictions on $\mathbf{F} / \Phi$ str constraints can be modeled in the Schema/Filter model of CON. A filter, the Feature Licensing Condition, can be used to rule out formally possible but apparently unattested positional faithfulness constraints for phonetically strong positions.

As noted in the preceding section, Steriade (1993, 1995, 1997, 1999ab) demonstrates that the special licensing abilities of syllable onsets/released consonants are restricted to features whose salient cues are found in the consonantal release, such as place, voicing, or aspiration. Consonants of this type have no special ability to license features such as retroflexion or preaspiration, since the cues to those features are found at the transition into a consonant, and simply being released does not mean that a consonant is in an environment (i.e., postvocalic) where cues for retroflexion and preaspiration are found.

A similar result emerges when the positional licensing effects compiled by Beckman (1998) for the phonetically strong position stressed syllable and the psycholinguistically strong position initial syllable are compared, as in (51). 
(51) Feature licensing by ó and $\sigma_{1}$ (from Beckman 1998)

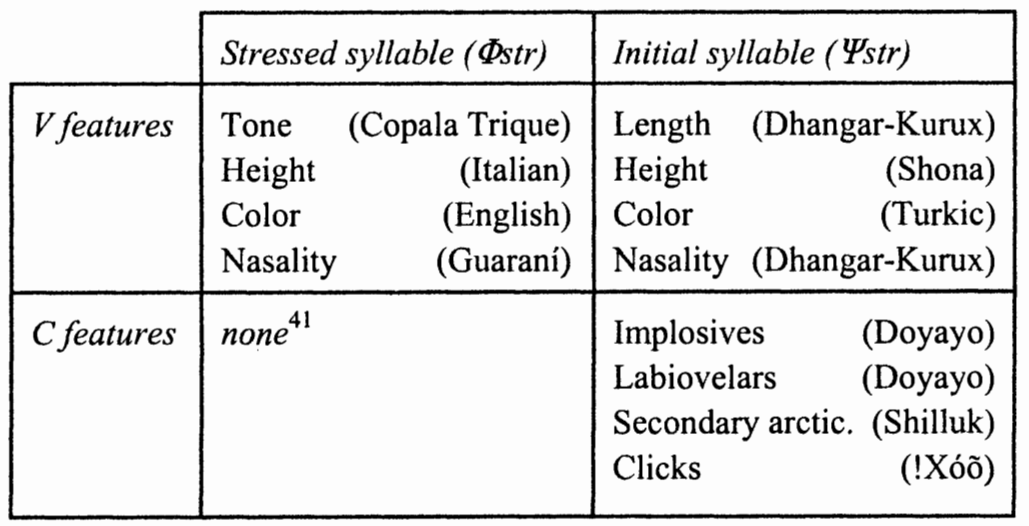

This chart supports the claim that a phonetically strong position can only have a special faithfulness relationship with a feature for which that position characteristically possesses salient cues. Since the phonetic prominence of a stressed syllable has to do with the duration, amplitude, and/or pitch contour of its rime, all of which increase the perceptibility of vowel features, there are stressedsyllable positional faithfulness constraints for vowel features but not for consonantal features. However, the initial syllable, a psycholinguistically strong position, has no such restriction; a variety of contrasts, involving both consonant and vowel features, are licensed by initial syllables in the languages listed in (51).

Although there is a close relationship between a phonetically strong position and the features for which it has a special licensing ability, there is reason to believe that this relationship is not a direct one. First, evidence from positional augmentation in phonetically strong positions shows that the status of 'phonetically strong position' is an abstract formal property that can be manipulated by the phonology in ways that are unrelated to the phonetic source of the position's special status. As discussed in $\S 2.3 .3$ and $\$ 2.4 .2 .2$ above and in Chapter 3, an augmentation

41. Actually, Beckman (1998) does list one example that might fit in this box: the Otomanguean language Copala Trique, which has final stress. The description of this language in Hollenbach (1977) suggests that certain consonant types are restricted to the onset of the final syllable, but there also appear to be allophonic alternations (suggestive of particularly low-ranking faithfulness) that occur only in this position as well. Further information is needed to determine whether it is the presence of stress per se that influences these consonant patterns; the otherwise striking restriction of Beckman's (1998) stressedsyllable licensing examples to vocalic features suggests that another explanation may be available for Copala Trique. 
constraint can be relativized to a phonetically strong position even when the prominent property manipulated by the augmentation constraint is not related to the characteristic phonetic salience of the position. For example, even though the stressed syllable is phonetically salient based on its vowel- or rime-related properties, this strong position is affected by positional augmentation constraints like ONSET/o and [ ${ }^{*}$ ONSET/X]/o, which do not affect vowels or rimes. This fact indicates that the formal phonological system has access to the abstract information $\sigma$ is a phonetically strong position, rather than to the phonetically transparent information ó has good vowel-and rime-based cues.

Second, the filters that have been discussed so far-the Prominence Condition, the Segmental Contrast Condition, and also the Inductive Grounding Principle of Hayes (1999a)-all show that substantive considerations influence the contents of CON through constraint filters that use extra-phonological information, from domains such as perception, processing, and articulation, to accept or reject formally possible constraints. This supports the proposal, advanced also by Hayes (1999a), that constraint construction itself is a formal process that freely generates constraints without regard to functional or substantive considerations-such substantive considerations being imposed exclusively by the constraint filters. If it is true that constraint construction is a purely formal process, then there is no way for the constraint-construction module to know that a positional faithfulness constraint like IDENT[Vheight]/o is a possible constraint, but something like IDENT[voice]/ó is not.

Thus, if the relationship between phonetically strong positions, like the stressed syllable, and the features for which they have special licensing abilities, in this case vowel-related features, is not supplied directly by the phonetics, then there must be some explanation for the fact that positional faithfulness constraints for phonetically strong positions are restricted to those involving features whose perceptual cues are particularly salient in the position in question. This is precisely the role played by filters in the Schema/Filter model. A full account of the restricted positional licensing behavior shown by phonetically strong positions would therefore involve an additional filter, the Feature Licensing Condition, stated informally as in (52).

Feature Licensing Condition

In a constraint of the form IDENT[Feat $] / \Phi$ str, the following condition must be met:

$\Phi$ str must possess salient cues for the perception of Feat. 
An examination of the contrasts listed in Beckman (1998) that are specially maintained by the psycholinguistically strong position root in the face of positional neutralization indicates that, as for the initial syllable, there are no restrictions on positional faithfulness constraints for this position. Thus, in general, there is nothing like the Feature Contrast Condition for psycholinguistically strong positions, and as a result, these positions show special licensing abilities for consonantal features as well as for vocalic features.

Feature licensing by root and $\sigma_{1}$ (Beckman 1998)

\begin{tabular}{|c|c|c|}
\hline & Root & Initial syllable \\
\hline $\begin{array}{l}V \\
\text { features }\end{array}$ & $\begin{array}{r}\text { (German) } \\
\text { (Turkish) }\end{array}$ & $\begin{array}{lr}\text { Length } & \text { (Dhangar-Kurux) } \\
\text { Height } & \text { (Shona) } \\
\text { Color } & \text { (Turkic) } \\
\text { Nasality } & \text { (Dhangar-Kurux) }\end{array}$ \\
\hline $\begin{array}{l}\text { C } \\
\text { features }\end{array}$ & $\begin{array}{lr}\begin{array}{l}\text { Non-coronal } \\
\text { place }\end{array} & \text { (German) } \\
\text { Affricates } & \text { (German) } \\
\text { Clicks } & \text { (Zulu, Xhosa) } \\
\text { Laryngeal } & \text { (Cuzco } \\
\text { features } & \text { Quechua) } \\
\text { [pharyngeal] } & \text { (Arabic) } \\
\text { Onset clusters } & \text { (Sanskrit) }\end{array}$ & $\begin{array}{lr}\text { Implosives } & \text { (Doyayo) } \\
\text { Labiovelars } & \text { (Doyayo) } \\
\text { Secondary arctic. } & \text { (Shilluk) } \\
\text { Clicks } & \text { (!Xóõ) }\end{array}$ \\
\hline
\end{tabular}

Thus, the distinction between phonetically and psycholinguistically strong positions is relevant for positional faithfulness constraints as well as for positional augmentation constraints. The Schema/Filter model of CON can provide an account for the special restrictions on positional IDENT constraints for phonetically strong positions with the Feature Licensing Condition, a constraint filter that ensures that a phonetically strong position only licenses features with which it has a special perceptual relationship.

\subsubsection{Summary}

Phonetically and psycholinguistically strong positions have different substantive bases for their special status. As a consequence, constraint filters, which are the locus of substantive restrictions on phonology, are potentially sensitive to the 
distinction between the two classes of strong positions. Indeed, the special relationship between psycholinguistically strong positions and early-stage word recognition places restrictions on possible augmentation constraints for these positions, as modeled in the Segmental Contrast Condition (further discussion of the psycholinguistic factors formalized in this constraint filter is provided in \$4.3). Another example has also been given preliminary consideration here: the special relationship between the particular phonetic salience of a phonetically strong position and the types of contrasts for which the position has $\mathbf{F} / \mathbf{s t r}$ constraints can also be modeled in terms of a substantively based constraint filter.

\subsection{Summary and conclusions}

This chapter has developed a formal theory of $\mathbf{M} / \mathbf{s t r}$ constraints. The Schema/Filter model of CON provides a way for substantive pressures to affect the universal inventory of constraints by means of constraint filters. A given constraint filter blocks the inclusion of any constraint in CON that fails to exhibit the particular substantively-based characteristic to which that filter is sensitive. Thus, the Prominence Condition rejects any $\mathbf{M} / \mathbf{s t r}$ constraint whose satisfaction does not enhance perceptual prominence, and the Segmental Contrast Condition rejects any $\mathbf{M} / \Psi$ str constraint whose satisfaction affects segmental contrasts (other than those that help demarcate the left edge of the initial syllable).

It is important to note that the Schema/Filter model of CON does not automatically predict that all constraints in CON have some relationship to substantive factors (although such a claim certainly could be incorporated into the model). If no filter exists to reject a particular type of constraint, then even if that constraint has no substantive basis, it will not be prevented from appearing in CoN.

Likewise, the approach to $\mathbf{M} / \mathbf{s t r}$ constraints outlined here does not automatically predict that an $\mathbf{M} / \mathbf{s t r}$ constraint exists for any phonetic characteristic that happens to be perceptually prominent. Filters do not create constraints; they merely evaluate, on the basis of some substantive factor, the constraints that are formally constructed from the set of constraint schemas and the set of basic phonological elements. Thus, if there happens to be some phonetic property that is perceptually salient, but is not part of the formal phonological system (as, e.g., a feature), then no $\mathbf{M} / \mathbf{s t r}$ (or other) constraint involving that property will ever be constructed in the first place. Instead, what the theory predicts is that if there is a constraint requiring the presence of property $P$, and $P$ happens to be a prominent property, then the constraint should have $\mathbf{M} / \mathbf{s t r}$ counterparts. 


\section{Augmentation of \\ Phonetically Strong Positions}

\subsection{Introduction}

This chapter presents a number of examples of positional augmentation in phonetically strong positions. As defined in Chapter 1 (following Beckman 1998), phonetically strong positions are those that characteristically contain salient cues to the perception of certain phonological contrasts; they are distinguished from psycholinguistically strong positions, which are examined in the following chapter. The phonetically strong positions for which augmentation effects are documented here are the positions stressed syllable (\$3.2), long vowel (\$3.3), and syllable onset or released consonant $(\$ 3.4)$.

The examples discussed in this chapter show that there are a variety of positional augmentation constraints that make reference to these three positions, including constraints calling for such properties as syllable weight, high-sonority nuclei, low-sonority onsets, high tone, and supralaryngeal place features. These properties have all been shown to enhance perceptual prominence (\$2.3.2), so constraints that require the presence of these properties are all legitimate $\mathbf{M} / \mathbf{s t r}$ constraints according to the Prominence Condition.

After the language examples have been presented in $\S \S 3.2-4$, a summary of the $\mathbf{M} / \mathbf{s t r}$ constraints discussed in this chapter is given in $\S 3.5$. As noted in $\$ 2.3 .3$ above, the typology of attested $\mathbf{M} / \mathbf{s t r}$ constraints for phonetically strong positions 
matches the predicted typology of such constraints as restricted only by the Prominence Condition. ${ }^{1}$

\subsection{Positional augmentation in stressed syllables}

A special relationship between stress and perceptual prominence is frequently recognized. For one thing, the phonetic realization of stress often involves one or more prominent properties, including pitch, duration, and amplitude (Lehiste 1970). Other perceptually prominent properties that stressed syllables are sometimes altered in order to manifest include low-sonority onsets (de Lacy 2000, to appear) or onsets in general. Furthermore, there are languages with what are traditionally called "unbounded stress systems," in which stress is attracted to a syllable that is intrinsically associated with a prominent property such as high tone, syllable weight, or vowel sonority (for recent discussion see Kenstowicz 1994, Hayes 1995, Walker 1996, and de Lacy 1999); attraction of stress to syllables with onsets or with lowsonority onsets has also been documented (Strehlow 1942; Davis 1988; Everett \& Everett 1984ab, Everett 1988).

The stressed syllable is a strong position, as evidenced by the resistance of stressed syllables to positional neutralization effects (Trubetzkoy 1939; Steriade 1993; Beckman 1998). The theory developed here thus predicts that there should be augmentation constraints-prominence-enhancing markedness constraintsrelativized to this position. Moreover, stressed syllables, being syllables, are large enough to encompass consonants, vowels, and prosodic features such as tone. Therefore, augmentation constraints that require a particular prominent property to hold of consonants or vowels, or that manipulate tone, can potentially be relativized to stressed syllables. In accordance with this prediction, there are indeed a wide range of augmentation effects observed in stressed syllables. For example, they are required to be heavy (Mohawk, West Germanic, Aguacatec; $\$ 3.2 .1 .1$ ), to bear high tone (Slave, Golin, Serbo-Croatian; §3.2.1.2), to have high-sonority nuclei (Slovene, Mokshan Mordwin; §3.2.1.3), to have onsets (Dutch, Western Arrernte; $\S 3.2 .2 .1$ ), or to have specifically low-sonority onsets (Niuafo'ou, Pirahã; §3.2.2.2).

The observed affinity of stressed syllables for prominent properties is thus accounted for by $\mathbf{M} / \boldsymbol{\sigma}$ constraints, prominence-enhancing markedness constraints relativized to stressed syllables. Under this approach, it is no accident that the kinds

1. The situation is different with psycholinguistically strong positions (considered in Chapter 4), because an additional filter, the Segmental Contrast Condition, is relevant for these positions. 
of properties involved in stress attraction (as in unbounded stress systems) are also those involved in stressed-syllable alteration effects (when syllables, independently designated to bear stress either by lexical specification or through the interaction of metrical constraints, are also required to have particular prominent properties). The same inventory of $\mathbf{M} / \mathbf{\sigma}^{\sigma}$ constraints is responsible for both classes of phenomena.

This section is structured as follows. First, in $\$ 3.2 .1$, familiar cases of stress/prominence interaction involving syllable weight, high tone, and nuclear sonority are briefly reviewed, and the $\mathbf{M} / \mathbf{\sigma}$ constraints responsible for such effects are introduced and exemplified. The relationship between stressed-syllable alteration and stress attraction phenomena is also discussed in this section (see especially §3.2.1.1). §3.2.2 then examines onset-related phenomena specific to stressed syllables. Interactions between stress and onsets or onset sonority levels are less common than the kinds of stress/prominence interactions discussed in $§ 3.2 .1$, so the languages introduced in $\$ 3.2 .2$ are discussed in somewhat greater depth. Crucially, the addition of an onset and the reduction of onset sonority are also ways to enhance perceptual prominence (as argued in §2.3.2.3), so positional versions of markedness constraints calling for these properties are correctly predicted to exist. The results of the section are summarized in $\$ 3.2 .3$.

\subsubsection{Familiar stress/prominence interactions through $M / \sigma o \sigma$ constraints}

This section reviews a number of interactions between the strong position stressed syllable and several properties that are traditionally regarded as perceptually prominent: syllable weight (\$3.2.1.1), high tone (\$3.2.1.2), and high-sonority syllable nuclei (\$3.2.1.3). The constraints responsible for these effects are all given formulations as $\mathbf{M} / \sigma$ constraints. The connection between stressed-syllable alteration and stress attraction effects is also examined.

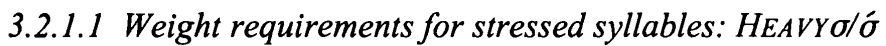

One of the most familiar examples of a requirement that holds of the strong position stressed syllable is seen in the special relationship between stress and heavy syllables, frequently discussed in the context of metrical stress theory. In some languages, such as Mohawk (Michelson 1988) or West Germanic (Murray \& Vennemann 1983), stressed syllables are required to be or become heavy, through vowel lengthening or consonant gemination. In other languages, with what are traditionally known as unbounded stress systems, including for example Aguacatec 
(McArthur \& McArthur 1956; Walker 1996), stress is attracted to already existing heavy syllables.

Both of these patterns represent ways in which a language can satisfy a single positional augmentation constraint on stressed syllables, HEAVYo/ó.

As discussed in Chapter 2, this constraint is a positional version, relativized to stressed syllables, of a constraint that requires syllables in general to be heavy. The formulation of HEAVYo/ó, as for any positional augmentation constraint, is compositional; it is predictable given the formulations of the $\mathbf{C} /$ str schema and the general constraint HEAVYo. ${ }^{2}$

$\mathrm{C} / \mathrm{str}$ For all $y$, if $y$ is a str, then $\mathbf{C}$ holds of $y$

where $y$ is an element in the focus of the constraint $\mathbf{C}$ (Crowhurst \& Hewitt 1997; see \$2.2.2)

HEAvYo

For all syllables $x, x$ dominates $>1$ mora

HEAVYo/ó is high-ranking and active in both stressed-syllable alteration languages like Mohawk or West Germanic and stress-attraction languages like Aguacatec. The difference between the two kinds of languages stems from the relative ranking of two other types of constraints. If the constraints that determine the location of stress (which may, depending on the language, be alignment constraints, faithfulness constraints, or both) outrank the faithfulness constraints regulating syllable weight, then faithfulness is what is violated, so stressed-syllable alteration occurs. But if the weight-related faithfulness constraints outrank the stress-location constraints, then the location constraints are violated, and the actual location of the syllable that bears stress will be determined by the location of any pre-existing heavy syllables in the prosodic word.

For example, consider the case of a language with an unbounded stress system that prefers to stress bimoraic syllables over monomoraic syllables when possible. One example of such a language is Aguacatec, given an OT analysis by Walker (1996) based on the original data and description in McArthur \& McArthur (1956) and analyses by Hayes $(1981,1995)$.

2. See $\$ 2.3 .2 .1$ on the status of HEAVYo as an independently attested general (i.e., nonpositional) constraint. 
In Aguacatec, when a word has only light syllables, stress falls on the rightmost syllable. (CV and CVC syllables are light; CV: syllables are heavy.)

Rightmost stress in Aguacatec (McArthur \& McArthur 1956:73-4)

$\begin{array}{llll}\text { wuqán } & \text { 'my foot' } & \text { ta?ál } & \text { 'its juice' } \\ \text { nkJk'a?tJán } & \text { '(someone) fed you' } & \text { ?awéj } & \text { 'your trousers' } \\ \text { bijól } & \text { 'butcher' } & \text { ?alk'óm } & \text { 'thief } \\ \text { pa?tbíl } & \text { 'raincape' } & \text { tfinhojlíh-ts 'they search for me' } \\ \text { ?at'új } & \text { 'your small earthen jug' } & & \end{array}$

However, if there is a heavy syllable, it receives stress, even when it is not the rightmost syllable in the word.

Heavy syllables preferentially stressed (McArthur \& McArthur 1956:73-4)

$\begin{array}{llll}\text { (a) Tintá: } & \text { 'my father' } & \text { (b) } \text { ?á:k'ah } & \text { 'new' } \\ \text { Tatsí:? } & \text { 'your dog' } & \text { ?á:ts'um } & \text { 'salt' } \\ \text { Tatsú:ts } & \text { 'your milk' } & \text { nqé:rats } & \text { 'that isn't it' } \\ & & \text { Té:q'um } & \text { 'carrier' } \\ & & \text { tfí:'bah } & \text { 'meat' } \\ & & \text { mí:tu? } & \text { 'cat' }\end{array}$

Previous OT treatments of unbounded stress systems, such as Kenstowicz (1994), Walker (1996), and Bakovic (1998), have demonstrated that the essential constraint interaction is one in which an alignment constraint requiring stress to fall at the left or right edge of the word is dominated by a constraint requiring stressed syllables to be heavy, ${ }^{3}$ which under the constraint nomenclature conventions adopted here is the positional augmentation constraint HEAVYo/o. As a result of this ranking, stress will fall on a heavy syllable when one is available, even if it is not

3. In default-to-opposite-side unbounded stress systems (where the heavy syllable nearest one edge is stressed, but in the absence of heavy syllables a light syllable at the other edge is stressed), additional constraints are involved. Zoll (1996, 1997a) develops an account of default-to-opposite stress in which COINCIDE constraints are used to locate light stressed syllables (as marked structures) at specified edges. Gordon (2000) argues that the only true cases of default-to-opposite systems are those of the "rightmost heavy, else leftmost" variety, and that intonational factors are responsible for the appearance of initial stress in words with only light syllables. 
the rightmost syllable in the word. (The effect of the alignment constraint, although it is dominated, is still seen when there are no heavy syllables in the word and stress is final, or when there is more than one heavy syllable in a word and the rightmost of them is chosen. ${ }^{4}$ See Walker (1996) for detailed discussion.)

HEAVYo/ó For all syllables $x$, if $x$ is a ó, then $x$ dominates $>1$ mora

ALIGN-R(ó, PrWd) $\quad \forall$ ó $\exists$ PrWd such that the Right edges of $\sigma^{\prime}$ and PrWd are aligned (McCarthy \& Prince 1993a) ('Stress is rightmost in the prosodic word')

(6) Heavy syllable chosen over rightmost syllable

\begin{tabular}{|c||c|c|}
\hline /mi:tur/ & HEAVYo/ó & ALIGN-R(ó, PrWd) \\
\hline \hline a. mistú? & $* !$ & \\
\hline b. mítu? & & \\
\hline
\end{tabular}

Another ranking relationship must also hold to produce a stress system like that in Aguacatec: $\operatorname{FAITH}(\mu)$, the faithfulness constraint that prohibits lengthening of an underlying short vowel, must also dominate the alignment constraint. Otherwise, final stress could be achieved without violating HEAVYo/ó simply by lengthening the final vowel of the word.

(7) Misalignment preferred over vowel lengthening in Aguacatec

\begin{tabular}{|r|c|c|c|}
\hline /mi:tur/ & HEAVYo/ó & FAITH $(\mu)$ & ALIGN-R(ó, PrWd) \\
\hline \hline a. mistú:? & & $* !$ & \\
\hline b. míttu? & & & \\
\hline
\end{tabular}

However, it is not logically necessary that $\operatorname{FAITH}(\mu)$ dominate ALIGN-R(ó, PrWd) in every language. The two constraints are completely independent, so the factorial typology of possible language systems includes a ranking in which these

4. McArthur \& McArthur (1956) do not provide any actual examples of words with two long vowels, but their description of the stress-assignment pattern is explicit. 
two constraints are reversed. Such a language would lengthen an underlyingly short final vowel in order to perfectly satisfy both the augmentation constraint HEAVYo/ó and the stress-location constraint ALIGN-R( $\sigma$, PrWd).

Vowel lengthening preferred over misalignment (hypothetical language)

\begin{tabular}{|c|c|c|c|}
\hline /mi:tur/ & HEAVYo/ó & $\begin{array}{l}\text { ALIGN-R } \\
\left({ }^{\circ}, \text { PrWd) }\right.\end{array}$ & $\operatorname{FAITH}(\mu)$ \\
\hline a. mi:tú:? & & & * \\
\hline b. mistu? & & $* !$ & \\
\hline
\end{tabular}

Indeed, there are languages that require stressed syllables to be heavy. One example of such a language is Mohawk (Iroquoian; Michelson 1988), in which long vowels are not generally permitted, but stressed open syllables are realized as long. ${ }^{5}$

Stressed-syllable lengthening in Mohawk (Michelson 1988:53)

\begin{tabular}{|c|c|c|}
\hline k-atirút-ha? & 1A-pull-HAB & 'I pull' \\
\hline$\wedge$-k-atirúz.t-ı? & FUT-1 A-pull-PUNC & 'I'll pull' \\
\hline \multicolumn{3}{|l|}{ /ohar/ } \\
\hline k-ohár-ha? & 1A-attach- $\mathrm{HAB}$ & 'I attach it' \\
\hline ^-k-ohá:.r-^? & FUT-1 A-attach-PUNC & 'I'll attach it \\
\hline
\end{tabular}

5. In Mohawk, regular penultimate stress assignment is disrupted by epenthesis; an epenthetic vowel in an open penult is ignored for stress assignment, giving rise to stress on the antepenult (or an even further leftward syllable if there are multiple epenthetic vowels). If the epenthetic vowel is the morphologically mandated "joiner vowel" [a], then the vowel in a stressed open antepenult will also be long, just as for regular penultimate stress. However, in the case of phonologically, rather than morphologically, determined epenthesis, the epenthetic vowel is realized as [e] and the vowel in a stressed open antepenult will, exceptionally, not be long. See Michelson (1988), especially chapters 5 and 6, for discussion.

Proto-Lake-Iroquoian, from which Mohawk has developed, had predictably long vowels in all stressed open syllables, whether penult or antepenult and whether epenthetic or underlying, except when the onset of the following syllable was a laryngeal consonant (Michelson 1988:52). 


\begin{tabular}{|c|c|c|}
\hline yo-tewey-awák-ht-ha? & NP-fan-shake-INST-HAB & 'fan' \\
\hline wak-atewey-awá:.k-u & 1P-fan-shake-STAT & 'I'm fanning myself' \\
\hline & & \\
\hline $\begin{array}{l}\text { ye-hyatu-hkhwa? } \\
\text { k-hyá:.tu-s }\end{array}$ & $\begin{array}{l}\text { FA-write-INST.HAB } \\
\text { 1A-write-HAB }\end{array}$ & $\begin{array}{l}\text { 'pencil' } \\
\text { 'I write' }\end{array}$ \\
\hline
\end{tabular}

Another example of a system in which the satisfaction of HEAVYo/ó affects not stress location, but syllable shape, can be found in the history of Germanic (Murray \& Vennemann 1983:525-526). Reconstructed syllable structure for Proto-Germanic includes forms like CV́x.yV, where $x$ and $y$ are consonants and $x$ has lower sonority than $y$, violating the common cross-linguistic tendency to avoid increasing sonority across a syllable boundary (Murray \& Vennemann's "Syllable Contact Law"). Furthermore, in West Germanic this changed, not simply to resyllabified CV́. $x y \mathrm{~V}$, but to $\mathrm{C} v x \cdot x y \mathrm{~V}$ with gemination. To explain both the marked Proto-Germanic syllabification and also the development of gemination rather than simple resyllabification in West Germanic, Murray \& Vennemann propose a "Stressed Syllable Law," which states, "The preferred stressed syllable (in Germanic) has exactly two morae." ${ }^{6}$ Their Stressed Syllable Law corresponds to the positional augmentation constraint HEAVYo/ó. ${ }^{7}$

Thus, two familiar kinds of stress-related phenomena-stressed-syllable alteration, as in Mohawk and West Germanic, and stress attraction, as in Aguacatec - can be attributed to the same positional augmentation constraint,

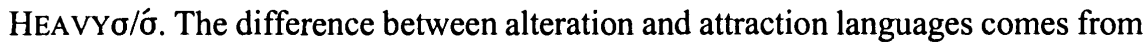
the relative ranking of the constraint or constraints that determine the location of stress (alignment constraints, faithfulness constraints, or both) with respect to the faithfulness constraints on syllable weight (such as a constraint against vowel

6. See also Lahiri \& Dresher (1999) on Open Syllable Lengthening in descendants of West Germanic. They argue that Open Syllable Lengthening was specifically a lengthening of short vowels in stressed open syllables, analogous to the Mohawk case, and was not due to compensatory lengthening (contra, e.g., Minkova 1982).

7. In this example, the faithfulness constraint that must be dominated by HEAVYo/ó is not a constraint against vowel lengthening, but rather, a constraint against converting an underlying singleton consonant into a geminate. But the more general pattern is the same: a weight-related faithfulness constraint that would otherwise protect a phonological contrast between light and heavy syllables is ranked below both HEAVYo/ó and a stress-location constraint, so it is syllable weight that is altered in response to HEAvYo/ó. 
lengthening, or a constraint against consonant gemination). As always, it is the lower-ranked constraint that is violated.

Alteration versus stress attraction

(a) Alteration ranking: HEAVYo/ó, LOCATE(Stress) > FAITH

(b) Attraction ranking: HEAVYo/ó, FAITH $>>$ LOCATE(Stress)

The results obtained in this section can be extended to the analysis of other augmentation processes that affect stressed syllables. In general, given a highranking stressed-syllable augmentation constraint that demands prominent property $P$, languages in which the relevant faithfulness constraints are ranked low will alter stressed syllables so that they have $P$, whereas languages with low-ranking stresslocation constraints will place stress on a syllable that already has $P$. Evidence that this is the right approach to take for stressed-syllable alteration and attraction phenomena in general comes from the fact that the same properties are involved in both patterns $(11,12)$.

(11) Stressed-syllable alteration effects

(a) ó becomes heavy: Mohawk, West Germanic

(b) ó acquires high tone: Slave

(c) ó increases the sonority of its nucleus: Slovene

(d) ó epenthesizes an onset: Dutch

(e) ó rejects a high-sonority onset: Niuafo'ou

(12) Stress attraction effects

(a) Attracted to heavy $\sigma$ : Aguacatec

(b) Attracted to $\sigma$ with high tone: Golin, Serbo-Croatian

(c) Attracted to $\sigma$ with high-sonority nuclei: Mokshan Mordwin

(d) Attracted to $\sigma$ with onsets: Arrernte

(e) Attracted to $\sigma$ with low-sonority onsets: Pirahã

Because stressed-syllable alteration and stress attraction are both responses to the same augmentation constraints, the fact that these two phenomena are sensitive to the same set of properties-syllable weight, high tone, sonority, and the presence of onsets-is given a principled account. 
The additional stressed-syllable augmentation constraints whose effects are seen in the languages listed in (11) and (12) are given in (13) below; these constraints are addressed in the remainder of this section (\$\$3.2.1.2-3) and in $\S 3.2 .2$. (The general counterparts of these $\mathbf{M} / \mathbf{s t r}$ constraints have been introduced in $\$ 2.3 .2$.

(13) Additional M/óo constraints

(a) HTONE/o For all syllables $x$, if $x$ is a $\sigma$, then a tone-bearing $(\$ 3.1 .2)$ unit associated with $x$ bears high tone

(b) $\left[{ }^{*} \mathrm{PEAK} / \mathrm{X}\right] /$ ó $\quad$ For every segment $a$ that is the head of some $(\S 3.1 .3)$ syllable $x$, if $x$ is a $\sigma$, then $|a|>\mathrm{X}$

where $|y|$ is the sonority of segment $y$

$\mathrm{X}$ is a particular step on the segmental sonority scale

(c) ONSET/ó

For all syllables $x$, if $x$ is a ó, then $a \neq b$ $(\S 3.2 .1)$

where $a$ is the leftmost segment dominated by $x$ $b$ is the head of $x$

(d) $\left[{ }^{*}\right.$ ONSET $\left./ \mathrm{X}\right] / \sigma \quad$ For every segment $a$ that is the leftmost pre-moraic (§3.2.2) segment of some syllable $x$, if $x$ is a ó, then $|a|<\mathrm{X}$ where $|y|$ is the sonority of segment $y$

$\mathrm{X}$ is a particular step on the segmental sonority scale

In summary, the commonly observed interactions between stress and syllable weight in which stressed syllables are required to become heavy or stress is attracted to heavy syllables can be accounted for with the constraint HEAVYo/o. This constraint is one member of the set of $\mathbf{M} / \mathbf{\sigma}$ constraints, markedness constraints that are relativized to the strong position stressed syllable. The fact that languages can satisfy HEAVYo/ó in one of two ways, through stressed-syllable alteration or through stress attraction, is true of other $\mathbf{M} / \boldsymbol{\sigma}$ constraints as well. In general, languages with stressed-syllable alteration change input material (violating some faithfulness constraint) to satisfy an $\mathbf{M} / \sigma^{\prime}$ constraint without affecting stress placement; languages with stress attraction force stress to fall on a syllable that 
already satisfies $\mathbf{M} / \boldsymbol{\sigma}$, violating the stress-location constraints but maintaining faithfulness.

\subsubsection{High tone in stressed syllables: HTONE/ó}

Another perceptually prominent property that is often seen to have a special relationship with stress is high $(\mathrm{H})$ tone. For example, in the Hare dialect of Slave (Rice 1987), $\mathrm{H}$ tone is realized on stressed syllables, and in Golin (Bunn \& Bunn 1970; Hayes 1995; Walker 1996; de Lacy 1999) and Serbo-Croatian (Zec 1999), stress is attracted to syllables with $\mathrm{H}$ tone. According to the treatment of strongposition-specific phonological requirements being developed here, these phenomena occur when the positional augmentation constraint HTONE/ó is ranked high, so that stressed syllables are required to be associated with $\mathrm{H}$ tone.

HTONE/ó

For all syllables $x$, if $x$ is a ó, then a tone-bearing unit associated with $x$ bears high tone

Again, just as for HEAVYo/o, the difference between the stressed-syllable alteration pattern found in Slave and the stress attraction pattern found in Golin or SerboCroatian emerges from the different ranking of tonal faithfulness constraints and stress-location constraints in these languages.

The Hare dialect of Slave (Athapaskan; Rice 1987) has an underlying contrast between morphemes that have an associated $\mathrm{H}$ tone and those that do not. But, in verbs, the surface location of the $H$ tone (if any) is entirely determined by the location of the stressed syllable in the morphologically complex form.

The following forms, composed of a person/number prefix with no lexical $\mathrm{H}$ tone and a verb root, show that there is a contrast between verb roots with and without $\mathrm{H}$ tone. Nevertheless, the $\mathrm{H}$ tone surfaces on the prefix rather than on the root itself. (Vowels associated with $\mathrm{H}$ tone are underlined.)

(15) Tonal contrasts in Hare verbs (Rice 1987:240)
(a) weh-dzo
/dzo/
's/he trapped'
wéh-k'e
$/ k^{\prime}$ e/
's/he shot'
(b) nع-dõ
/dõ/
'you (sg.) drink'
$n \underline{\varepsilon}-? a$
/?a/
'you (sg.) eat' 

(c) heh-ji
/ti/
'I sing'
heh-lu
/lu/
'I net'

Verb roots are always monosyllabic; one or more prefixes may precede the root; at most one suffix syllable may follow the root; and the location where the $\mathrm{H}$ tone is realized is always the syllable immediately preceding the root.

According to Rice (1987), the syllable preceding the root is the location of stress. Rice justifies this claim on the basis of processes of vowel deletion and tenseness harmony that are sensitive to metrical structure (as well as on impressionistic evidence that the pre-root syllable has greater amplitude and/or duration than the root syllable even when no $\mathrm{H}$ tone is present). Therefore, the placement of $\mathrm{H}$ tone in Slave verbs is an example of a stressed-syllable alteration effect. As usual for an alteration pattern, both the stressed-syllable augmentation constraint HTONE/ $\sigma$ and the constraint(s) determining the location of stress, encapsulated here as LOCATE(Stress) ${ }^{8}$ must outrank the faithfulness constraint(s) against tone shift, represented here as NOSHIFT (16). To account for the fact that no $\mathrm{H}$ tone is epenthesized to satisfy HTONE/o when the input lacks a $\mathrm{H}$ tone, the ranking DEP-TONE >> HTONE/ó is also necessary (17).

(16) Stressed syllable is altered by attracting $\mathrm{H}$ tone

\begin{tabular}{|c|c|c|c|c|}
\hline /wعh + k'e/ & $\begin{array}{l}\text { DEP- } \\
\text { TONE }\end{array}$ & HTONE/ó & $\begin{array}{l}\text { LOCATE } \\
\text { (Stress) }\end{array}$ & NoShIFT \\
\hline a. wéhk'e & & $* !$ & & \\
\hline b. wénh'e & & & & * \\
\hline c. WEhk'é & & & $* !$ & \\
\hline
\end{tabular}

8. Rice (1987) accounts for stress location with a trochaic foot that is right-aligned with the verb root (since the addition of a suffix vowel does not affect the placement of stress). 
No H-tone epenthesis

(HTONE/ó violations tolerated when the input has no $\mathrm{H}$ tone)

\begin{tabular}{|r||c|c|c|c|}
\hline /weh + dzo/ & $\begin{array}{c}\text { DEP- } \\
\text { TONE }\end{array}$ & HTONE/ó & $\begin{array}{c}\text { LOCATE } \\
\text { (Stress) }\end{array}$ & NOSHIFT \\
\hline \hline a. wéhdzo & & $*$ & & \\
\hline b. wéhdzo & $* !$ & & & $*$ \\
\hline
\end{tabular}

Thus, whenever there is a $\mathrm{H}$ tone present in the input, as in (16), violation of lowranking NOSHIFT allows for the satisfaction of both the LOCATE(Stress) constraints and HTONE/o. That is, stressed syllables are altered so that they bear $\mathrm{H}$ tone.

Other languages in which stressed syllables are altered to bear $\mathrm{H}$ tone, providing further motivation for the positional augmentation constraint HTONE/ $\sigma$, include Safwa (Zoll 1997b) and Sukuma (Kang 1997). ${ }^{9}$

The other expected pattern-the attraction of stress to syllables intrinsically associated with $\mathrm{H}$ tone-is found, for example, in Golin (Chimbu; New Guinea). This language has been analyzed by Hayes (1995), Walker (1996), and de Lacy (1999), based on the description by Bunn \& Bunn (1970). Stress in Golin appears on the rightmost syllable with $\mathrm{H}$ tone, and on the final syllable in words with only $\mathrm{L}$ tones. (Again, vowels with high tone are underlined.)

Stress in Golin (Bunn \& Bunn 1970:5)

(a) Rightmost H-tone syllable

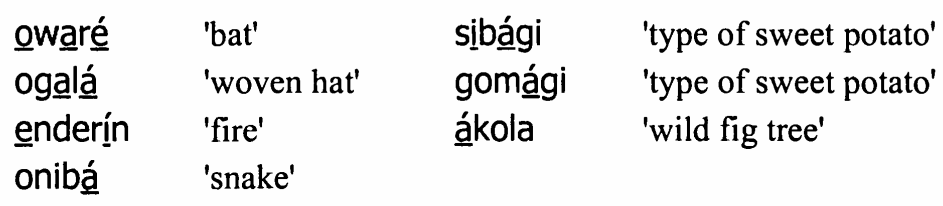

9. As noted in $\$ 2.3 .2 .5$, a complete account of the interaction between tone and strong positions would probably also include a constraint LTONE/ó. Sukuma is, according to Kang's (1997) analysis but in terms of the constraints developed here, an example of a language where HTONE/ó outranks LTONE/ó (this is presumably a universally fixed ranking, given the greater intrinsic prominence of $\mathrm{H}$ over L tones; see de Lacy 1999), but LTONE/ó is also active, with the result that a metrical head is supplemented with a $\mathrm{L}$ tone when a $\mathrm{H}$ tone is not available. Arguments for the affinity of stressed syllables for all tones, not just $\mathrm{H}$, are also presented in Yip (2000). 
(b) Final syllable (no H)

kawligí 'post'

These facts indicate that the positional augmentation constraint HTONE/ó dominates the stress-placement constraint ALIGN-R(ó, PrWd). As a result of this ranking, stress appears as far to the right as possible (19), but it must appear on a $\mathrm{H}$-tone syllable when there is one (20).

(19) Rightmost stress preferred when possible

\begin{tabular}{|c|c|c|}
\hline /oware/ & HTONE/ó & ALIGN-R(ó, PrWd) \\
\hline \hline a. ôwaré & & \\
\hline b. owwáre & & $* !$ \\
\hline c. ówąre & & $* ! *$ \\
\hline
\end{tabular}

(20) Attraction of stress to $\mathrm{H}$ tone takes priority over right-edge alignment

\begin{tabular}{|r||c|c|}
\hline /sịbagi/ & HTONE/ó & ALIGN-R(ó, PrWd) \\
\hline \hline a. sibagai & $* !$ & \\
\hline b. sịbági & & $*$ \\
\hline c. síbạgi & & $* * !$ \\
\hline
\end{tabular}

Another example of stress attraction to syllables with $\mathrm{H}$ tone is found in the Neo-Štokavian dialects of Serbo-Croatian (Zec 1999). Here, the placement of $\mathrm{H}$ tone in a form is largely determined by morphological factors (see Zec 1999 for discussion), but it is in turn the location of the $\mathrm{H}$ that determines the surface location of stress. To account for the attraction of stress to H tones, Zec (1999) proposes a constraint called SFOOTSALIENCE, formulated as in (21).

SFoOTSALIENCE $\quad$ Head $_{\text {PrWd }}$ has to be associated with a High tone (Zec 1999:251)

This constraint is clearly analogous to HTONE/o. 
Thus, the interaction between stress and $\mathrm{H}$ tone in the languages discussed above can be accounted for with the positional augmentation constraint HTONE/o. Moreover, both of the two possible ways of satisfying this constraint are attested. In Hare, stressed-syllable alteration effects are observed. The location of stress is fixed by high-ranking metrical constraints and never varies; instead, if there is a $\mathrm{H}$ tone present in the representation, it becomes affiliated with the stressed syllable so that the stressed syllable will bear $\mathrm{H}$ tone. On the other hand, Golin and SerboCroatian show the typical stress-attraction pattern. In these languages, the location of a $\mathrm{H}$ tone is determined by factors other than stress (faithfulness to lexical specification in Golin and constraints on morphological structure in SerboCroatian), and the location of stress is determined by the location of $\mathrm{H}$ tone.

\subsubsection{High-sonority nuclei in stressed syllables: $\left[{ }^{*} P E A K / X\right] / \sigma$}

The constraint subhierarchy that demands high-sonority syllable nuclei, [*PEAK/X], is also predicted to have a version specific to stressed syllables, since higher sonority nuclei are more perceptually prominent. And as predicted, the relativized constraint subhierarchy $\left[{ }^{*} \mathrm{PEAK} / \mathrm{X}\right] / \sigma$ is attested. Once again, languages in which members of the $\left[{ }^{*} \mathrm{PEAK} / \mathrm{X}\right] /$ o subhierarchy are active come in the predicted two varieties: stressed-syllable alteration languages such as Zabice Slovene, in which the nuclei of stressed syllables become higher in sonority, and stress-attraction languages like Mokshan Mordwin, in which stress placement is determined by the sonority of syllable nuclei.

Crosswhite (1999b), in a study of stress-related vowel-neutralization effects, reports a large number of languages that follow the familiar positional neutralization pattern: vowel qualities that are contrastive in stressed syllables undergo neutralization in unstressed syllables. However, there is one language reported in the study, the Zabice dialect of Slovene, that has vowel qualities that are contrastive in unstressed syllables but are neutralized in stressed syllables. Crucially, as predicted by the theory of $\mathbf{M} / \mathbf{s t r}$ constraints developed here, the vowels that are banned from stressed syllables are those of low sonority (low perceptual prominence); they are replaced in stressed syllables with vowels of higher sonority. 
Crosswhite, citing Rigler (1963), lists the following inventories of possible monomoraic nuclei in stressed and unstressed syllables in Zabice Slovene. As indicated, the high vowels $[i+\mathrm{u}]$ are banned from stressed syllables. ${ }^{10}$

(a) Unstressed syllable

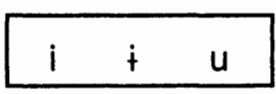

e $\partial 0$

a (b) Stressed syllable

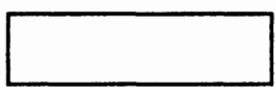

e $\quad$ o 0

a

Crosswhite (1999b) accounts for the ban on stressed high vowels with a constraint subhierarchy called *STRESSED/X, which is defined as a version of the ${ }^{*}$ PEAK/X subhierarchy making explicit reference to the peak of a stressed syllable. Crosswhite's proposed hierarchy is thus nearly equivalent to what would under the constraint naming conventions adopted here be called $\left[{ }^{*} \mathrm{PEAK} / \mathrm{X}\right] / \sigma^{.1}{ }^{11}$

10. A few additional facts about the vowel system of Zabiče Slovene are as follows. First, long high vowels [i: $\dot{t}: \mathrm{u}:]$ are permitted even when stressed. Also, there is a long vowel [ $\varepsilon:]$ that does not have a short counterpart. These two facts can both be accounted for with highranking positional faithfulness constraints on vowel features relativized to the strong position long vowel. These positional faithfulness constraints must outrank the stressed-syllable augmentation constraint [ $\left.{ }^{*} \mathrm{PEAK} / \mathrm{HIGHV}\right] / \sigma$ (see below) and the featural markedness constraint against the highly marked vowel quality $[\varepsilon]$, so that short versions of $[\varepsilon]$ and of high stressed vowels are prohibited, but long versions of these vowels are protected.

11. There are two differences between Crosswhite's (1999b) *STRESSED/X subhierarchy and the $\left[{ }^{*} \mathrm{PEAK} / \mathrm{X}\right] /$ ó subhierarchy as adopted here (see $\$ 2.3 .2 .2$ for detailed discussion of *PEAK/X). First, Crosswhite (1999b) follows Kenstowicz (1994) in postulating that ${ }^{*} \operatorname{PEAK}\left({ }^{*} \operatorname{STRESSED}\right) /[\theta]$ is the highest-ranking member of the ${ }^{*} \operatorname{PEAK}\left({ }^{*} \operatorname{STRESSED}\right) / \mathrm{X}$ subhierarchy. As discussed in $§ 2.3 .2 .2$, it is argued here that schwa patterns with other mid vowels in terms of its sonority, and the avoidance of stressed schwa that is observed in many languages stems from factors other than sonority. Second, in order to allow stressed high vowels to surface when long, Crosswhite (1999b) stipulates that the ${ }^{*}$ STRESSED/X subhierarchy refers explicitly to monomoraic vowels. Here, it is proposed that the [*PEAK/X]/ó subhierarchy is not sensitive to mora count, but only to vowel quality; the appearance of long stressed high vowels in Zabiče Slovene can be independently accounted for by means of a positional faithfulness constraint that protects the feature [+high] in the strong position long vowel. 
(23) $\left[{ }^{*} \mathrm{PEAK} / \mathrm{X}\right] / \sigma \quad$ For every segment $a$ that is the head of some syllable $x$, if $x$ is a ó, then $|a|>\mathrm{X}$

where $|y|$ is the sonority of segment $y$

$\mathrm{X}$ is a particular step on the segmental sonority scale

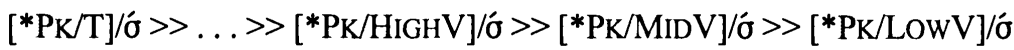

Crosswhite (1999b) does not present any actual examples from Zabiče Slovene in her discussion, but she gives the following description of her source, Rigler (1963).

\begin{abstract}
Although Rigler does not provide dialectal forms illustrating the relevant neutralizations, he indicates that in this dialect etymological short accented high vowels are realized as mid vowels: /í,, , > [é], /ú/ > [ó]. (Crosswhite 1999b:44)
\end{abstract}

The analysis here follows Crosswhite in assuming, based on the diachronic pattern that Rigler reports, that synchronically too, underlying short high vowels are realized as mid vowels when they are stressed. This means that [*PEAK/HIGHV]/o, which forbids high vowels in stressed syllables, dominates the faithfulness constraint that would otherwise prevent high vowels from being realized as mid, IDENT[high]. Also undominated, given that this is an alteration language, are the constraints that determine stress placement, once again encapsulated as LOCATE(Stress).

$\left[{ }^{*} \mathrm{PEAK} / \mathrm{HIGHV}\right] / \sigma \quad$ For every segment $a$ that is the head of some syllable $x$, if $x$ is a ó, then $|a|>\mathrm{HIGHV}$

where $|y|$ is the sonority of segment $y$

IDENT[high]

Output segments agree with their input correspondents in their specification for the feature [high] (McCarthy \& Prince 1995)

The interaction among these constraints is shown in (25). 
(25) Lowering of high vowels in stressed syllables (hypothetical input)

\begin{tabular}{|r||c|c|c|}
\hline /túka/ & LOCATE(Stress) & [*PEAK/HiV]/ó & IDENT[high] \\
\hline \hline a. túka & & $* !$ & \\
\hline b. tuká & $* !$ & & \\
\hline c. tóka & & & $*$ \\
\hline
\end{tabular}

Because it is not the case that all vowels become as high in sonority as possible when they appear in stressed syllables, we know that the next lower member of the [ $\left.{ }^{*} \mathrm{PEAK} / \mathrm{X}\right] / \sigma$ hierarchy, $\left[{ }^{*} \mathrm{PEAK} / \mathrm{MIDV}\right] / \sigma$, is dominated by IDENT[low], the faithfulness constraint that forbids changes in the specification of the feature [low] (on the assumption that mid vowels are [-high, -low]). Otherwise, underlying high and mid vowels would be realized as low vowels, rather than mid vowels, in stressed syllables. ([*PEAK/HIGHV]/ó and IDENT[low] do not conflict, so their ranking relationship cannot be directly established; the same is true of IDENT[high] and IDENT[low].)

[ $\left.{ }^{*} \mathrm{PEAK} / \mathrm{MIDV}\right] / \sigma \quad$ For every segment $a$ that is the head of some syllable $x$, if $\mathrm{x}$ is a ó, then $|a|>\operatorname{MIDV}$

where $|y|$ is the sonority of segment $y$

IDENT[low] Output segments agree with their input correspondents in their specification for the feature [low]

(McCarthy \& Prince 1995)

(27) Stressed vowels do not all become low (hypothetical input)

\begin{tabular}{|r||c|c|c|c|}
\hline /túka/ & {$\left[{ }^{*} \mathrm{PK} / \mathrm{HIV}\right] / \sigma$} & $\begin{array}{c}\text { IDENT } \\
\text { [high] }\end{array}$ & $\begin{array}{c}\text { IDENT } \\
{[\text { low] }}\end{array}$ & {$\left[{ }^{*} \mathrm{PK} / \mathrm{MIDV}\right] / \sigma$} \\
\hline \hline a. túka & $* !$ & & & \\
\hline b. tóka & & $*$ & & \\
\hline c. táka & & $*$ & $* !$ & \\
\hline
\end{tabular}


Members of the $\left[{ }^{*} \mathrm{PEAK} / \mathrm{X}\right] / \sigma$ subhierarchy are thus responsible for stressedsyllable alteration effects in languages like Slovene, where low sonority nuclei become higher in sonority under stress. As with the other M/ó constraints, members of the $\left[{ }^{*} \mathrm{PEAK} / \mathrm{X}\right] / \sigma$ hierarchy can also be responsible for stress-attraction effects, as seen in languages where stress is attracted to vowels that are already high in sonority. Such languages have been discussed by, among others, Hayes (1995) and Kenstowicz (1994). One example is the Mokshan dialect of Mordwin (Finno-Ugric; Kenstowicz 1994, citing data from Tsygankin \& Debaev 1975), in which stress is never assigned to high vowels if there are non-high vowels in a word. ${ }^{12}$

In Mokshan Mordwin, stress falls on the leftmost possible vowel but avoids high vowels. This pattern indicates that the left-alignment constraint for stress, Align-L( $\sigma$, PrWd), must be dominated by [*PEAK/HIGHV]/o (28). However, the fact that mid and low vowels are treated equivalently shows that ALIGN-L(ó, PrWd) dominates both [ $\left.{ }^{*} \mathrm{PEAK} / \mathrm{MIDV}\right] / \sigma$ and [*PEAK/LOWV]/o; that is, placing stress as close to the left edge as possible is preferable even to choosing a low vowel over a mid vowel (29).

(28) High vowels are avoided for stress: /putat/ 'you set down'

\begin{tabular}{|c||c|c|c|c|}
\hline /putat/ & $\begin{array}{c}{\left[{ }^{*} \mathrm{PK} /\right.} \\
\mathrm{HIV}] / \sigma\end{array}$ & ALIGN-L & $\begin{array}{c}{\left[{ }^{*} \mathrm{PK} /\right.} \\
\mathrm{MIDV}] / \sigma\end{array}$ & $\begin{array}{c}{\left[{ }^{* \mathrm{PK} /}\right.} \\
\mathrm{LOV}] / \sigma\end{array}$ \\
\hline \hline a. pútat & $* !$ & & & \\
\hline b. putát & & $*$ & & $*$ \\
\hline
\end{tabular}

12. Stress also avoids schwa in Mokshan Mordwin. As discussed in $\$ 2.3 .2 .2$, this fact is best accounted for with a separate constraint, orthogonal to the *PEAK/X subhierarchy. 
(29) Leftmost stress preferred, given a non-high vowel: /noldasak/ 'you release it'

\begin{tabular}{|r||c|c|c|c|}
\hline /noldasak/ & $\begin{array}{c}{\left[{ }^{*} \mathrm{PK} /\right.} \\
\text { HIV]/ó }\end{array}$ & ALIGN-L & $\begin{array}{c}{\left[{ }^{*} \mathrm{PK} /\right.} \\
\mathrm{MIDV}] / \sigma\end{array}$ & $\begin{array}{c}{\left[{ }^{*} \mathrm{PK} /\right.} \\
\mathrm{LoV}] / \sigma\end{array}$ \\
\hline \hline a. nóldasak & & & $*$ & \\
\hline b. noldásak & & $* !$ & & $*$ \\
\hline c. noldasák & & $* * !$ & & $*$ \\
\hline
\end{tabular}

In both Zabice Slovene and Mokshan Mordwin, the line between good and bad stressed-syllable peaks is drawn between mid and high vowels; that is, high vowels and everything lower in sonority are avoided as peaks in stressed syllables, while mid vowels and everything higher in sonority are selected. This division is the result of some other constraint (IDENT[low] in Slovene and ALIGN-L in Mordwin) dominating [ $\left.{ }^{*} \mathrm{PK} / \mathrm{MIDV}\right] / \sigma$ and rendering it inactive. However, there is nothing intrinsically important about the division between high and mid vowels, and in fact, there are stress systems in which other divisions of the sonority hierarchy are observed.

For example, English allows syllabic sonorants, [ $r \mid m \mathfrak{n}$ ], as nuclei in unstressed syllables. However, the options for stressed syllables are more limited: syllabic nasals are banned, and some dialects ban [l] as well, allowing only [r].

(30) Syllabic sonorants in English

(a) Unstressed syllables

(b) Stressed syllables

\begin{tabular}{|c|c|c|c|}
\hline maker & méj.kr & cur & kŕ \\
\hline able & éj.b! & bull & búl \\
\hline complain & kmp.pléjn & come & kím \\
\hline mason & méj.sn & sun & sín \\
\hline congratulate & kn.grǽdzəlèjt & hung & hín \\
\hline
\end{tabular}


That is, English makes a sonority division for stressed-syllable nuclei, not between high and mid vowels as in the previous examples, but between laterals and rhotics. ${ }^{13}$ This time, the constraint that crucially intervenes at some point along the [*PEAK/X]/ó subhierarchy (perhaps DEP-SEG, which would prevent the insertion of an epenthetic vowel to serve as the syllable nucleus) is ranked higher than in Slovene and Mordwin-dominating [ $\left.{ }^{*} \mathrm{PEAK} / \mathrm{RHO}\right] / \sigma$ and all the lower-ranked $\left[{ }^{*} \mathrm{PEAK} / \mathrm{X}\right] / \sigma$ constraints, rather than dominating only the last two $\left[{ }^{*} \mathrm{PEAK} / \mathrm{X}\right] / \sigma$

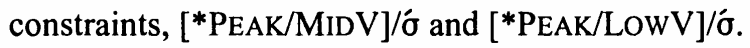

(31) Sonority division between rhotics and laterals for stressed-syllable nuclei

(a) Stressed [r] is permitted

\begin{tabular}{|c||c|c|c|c|}
\hline$/ \mathrm{kr} /$ cur & $\begin{array}{r}{\left[{ }^{*} \mathrm{PK} /\right.} \\
\mathrm{NAS}] / \sigma\end{array}$ & $\begin{array}{c}{\left[{ }^{*} \mathrm{PK} /\right.} \\
\mathrm{LAT}] / \sigma\end{array}$ & DEP-SEG & $\begin{array}{r}{\left[{ }^{*} \mathrm{PK} /\right.} \\
\mathrm{RHO}] / \sigma\end{array}$ \\
\hline \hline a. $\mathrm{kr}$ & & & & $*$ \\
\hline b. $\mathrm{kVr}$ & & & $* !$ & \\
\hline
\end{tabular}

(b) Stressed [1] not permitted (hypothetical input)

\begin{tabular}{|c|c|c|c|c|}
\hline$/ \mathrm{bl} /$ & $\begin{array}{c}{\left[{ }^{*} \mathrm{PK} /\right.} \\
\mathrm{NAS}] / \sigma\end{array}$ & $\begin{array}{c}{\left[{ }^{*} \mathrm{PK} /\right.} \\
\mathrm{LAT}] / \sigma\end{array}$ & DEP-SEG & $\begin{array}{r}{\left[{ }^{*} \mathrm{PK} /\right.} \\
\mathrm{RHO}] / \sigma\end{array}$ \\
\hline \hline a. 'b! & & $* !$ & & \\
\hline b. 'bVl & & & $*$ & \\
\hline
\end{tabular}

It should be noted that example (31b) is not intended to assert that the stressed vowel in the English word bull is epenthetic. No words with stressed nuclear [1] are possible in English, so even though no actual lexical entries probably contain such a structure, the grammar of English must ensure that if such an input were encountered, some unfaithful candidate that has eliminated the stressed nuclear [1]

13. This pattern is additional support for the sonority distinction between rhotics and laterals (see also §2.3.2.2 and §4.2.1.2). 
would be selected; this outcome is what (31b) demonstrates. See the discussion of richness of the base in $\S 1.3 .2$, especially footnote 12 .

Thus, as predicted given the Prominence Condition and the relationship of nuclear sonority to perceptual prominence, there is a positional version of the $\left[{ }^{*} \mathrm{PEAK} / \mathrm{X}\right]$ constraint subhierarchy for stressed syllables: [*PEAK/X]/o.

\subsubsection{Summary}

The Schema/Filter Model of CoN holds that any formally possible constraint is an actually occurring constraint as long as there are no substantively based constraint filters to rule it out. M/str constraints formed by relativizing markedness constraints to the phonetically strong position stressed syllable are subject only to one filter, the Prominence Condition. This filter passes $\mathbf{M} / \mathbf{s t r}$ constraints if they are formed from markedness constraints that require the presence of perceptually prominent properties. Therefore, this system predicts that $\mathbf{M} / \mathbf{\sigma}$ constraints requiring syllable weight, high tone, and high-sonority syllable nuclei should exist, since these are widely recognized examples of perceptually prominent properties.

In accordance with this prediction, the M/ó constraints HEAVYo/o, HTONE/ó, and the $[* \mathrm{PEAK} / \mathrm{X}] / \sigma$ subhierarchy are in fact attested. This section has reviewed familiar examples of interaction between stress and the perceptually prominent properties syllable weight, high tone, and high-sonority syllable nuclei, showing that these interactions are accounted for with the positional augmentation constraints given above. Furthermore, depending on the ranking of other constraints in the

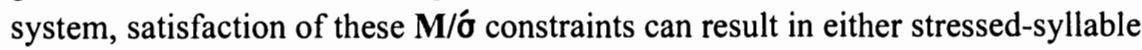
alteration or stress attraction effects. The fact that exactly the same properties are involved in alteration and attraction supports an approach, like this one, in which the same constraints are responsible for both classes of phenomena.

\subsubsection{Stress/onset interactions through $\mathrm{M} /{ }^{\prime}$ constraints}

As argued in $\$ 2.3 .2 .3$, syllables with onsets are more perceptually prominent than syllables without onsets, and the lower in sonority the onset, the more perceptually prominent the syllable. Markedness constraints requiring onsets and those requiring low-sonority onsets thus qualify as augmentation constraints and are predicted to have $\mathbf{M} / \mathbf{s t r}$ counterparts.

The languages discussed in this section show that ONSET and the members of the * ONSET/X constraint subhierarchy do, as predicted, have positional counterparts relativized to the strong position stressed syllable. As was the case for the $\mathbf{M} / \boldsymbol{\sigma}$ 
constraints considered in the preceding section, these onset-related $\mathbf{M} / \mathbf{\sigma}$ constraints give rise to both stressed-syllable alteration and stress attraction phenomena. The effects of ONSET/o, which requires stressed syllables to have onsets, are seen in Dutch (§3.2.2.1), where stressed syllables acquire epenthetic glottal-stop onsets, and in Western Arrernte (\$3.2.2.2), where stress is attracted to syllables with onsets. Likewise, the effects of the [ $\left.{ }^{*} \mathrm{ONSET} / \mathrm{X}\right] / \sigma$ subhierarchy, which enforces low sonority in onset consonants, are seen in Niuafo'ou (§3.2.2.3), where glide onsets are banned from appearing in stressed syllables, and in Pirahã (§3.2.2.4), where stress is attracted to syllables with voiceless obstruent onsets.

\subsubsection{ONSET/ó in Dutch: Glottal-stop epenthesis in stressed syllables}

In Dutch, as described by Booij (1995), onsetless syllables are avoided whenever possible. This fact can be attributed to a relatively high rank for the general markedness constraint ONSET. Nevertheless, unstressed syllables manage to surface without onsets if the only way for them to have an onset would be to epenthesize one, indicating that DEP-SEG dominates ONSET. Crucially, however, stressed syllables must always have an onset, even if this forces epenthesis. ONSET/ó, a stressed-syllable augmentation constraint requiring onsets specifically in stressed syllables, must therefore dominate DEP-SEG.

Given an underlying $/ V_{1}-V_{2}$ / sequence where $V_{1}$ is nonlow, hiatus in Dutch is always resolved by glide formation (32). The glide that appears agrees with $V_{1}$ in rounding and backness, indicating that the glide is created from $V_{1}$ (Rosenthall 1994). Glide formation occurs regardless of the surface stress pattern of the word.

(32) Glide formation after nonlow $\mathrm{V}_{1}$ (Booij 1995, (23))

(a) [4] after rounded front vowels

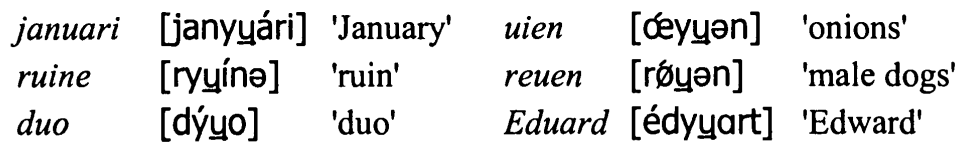

(b) [j] after unrounded front vowels

$\begin{array}{llllll}\text { dieet } & \text { [dijét] } & \text { 'diet' } & \text { Gea } & \text { [yéja] } & \text { (fem. name) } \\ \text { bioscoop } & \text { [bijoskóp] } & \text { 'cinema' } & \text { zeeen } & \text { [zéjən] } & \text { 'seas' } \\ \text { Indriaas } & \text { [Indrijas] } & \text { 'Andrew' } & \text { vijand } & \text { [VÉijant] } & \text { 'enemy' }\end{array}$


(c) [U] after nonlow back (=round) vowels

\section{Ruanda [ruvánda] 'Rwanda' \\ houen [hóuven] 'to hold'}

The appearance of an onset glide homorganic to $V_{1}$ in the forms in (32) indicates that the input vowel has two output correspondents - the vowel and the glide. Such output forms violate INTEGRITY (McCarthy \& Prince 1995), the faithfulness constraint against multiple output correspondents, but in doing so they satisfy ONSET while also avoiding the DEP-SEG violation that would be incurred by a truly epenthetic onset (a segment with no input correspondent).
ONSET
For all syllables $x, a \neq b$
where
$a$ is the leftmost segment dominated by $x$
$b$ is the head of $x$
DEP-SEG
Output segments have input correspondents (McCarthy \& Prince 1995)
INTEGRITY Input segments do not have multiple output correspondents (McCarthy \& Prince 1995)

The interaction among these three constraints for a word like those in (32) is shown in (34). Numerical subscripts indicate correspondence relations between input and output segments; for readability, only the crucial correspondence relations are shown in the output forms. Epenthetic segments (with no input correspondents) are in boldface.

Glide formation to provide onsets

\begin{tabular}{|c|c|c|c|}
\hline$/ \mathrm{d}_{1} \mathrm{i}_{2} \mathrm{e}_{3} \mathrm{t}_{4} \mid$ & DEP-SEG & ONSET & INTEGRITY \\
\hline \hline a. $\mathrm{di}_{2}$ et & & $* !$ & \\
\hline b. $\mathrm{di}_{2} \cdot \mathrm{r}_{5}$ et & $* !$ & & \\
\hline c. $\mathrm{di}_{2} \cdot \mathrm{j}_{2}$ et & & & $*$ \\
\hline
\end{tabular}

The facts discussed so far are true of any instance of hiatus in which $V_{1}$ is a nonlow vowel, independent of stress. Stress becomes relevant when $V_{1}$ is the low 
vowel [a]. In an [a]- $\mathrm{V}_{2}$ sequence, no glide is formed (low vowels are often dispreferred as glides; see, e.g., McCarthy \& Prince 1993b, Rosenthall 1994). If the potentially onsetless syllable (the one containing $V_{2}$ ) is not stressed, then it simply surfaces without any onset (35).

[a] $-V_{2}$ hiatus, where $V_{2}$ is not stressed (Booij 1995, (22))

$\begin{array}{lll}\text { chaos } & \text { [xá.ôs] } & \text { 'chaos' } \\ \text { farao } & {[\text { fá.ra.o] }} & \text { 'pharaoh' }\end{array}$

The above examples show that the constraint that disfavors low glides, here informally called *LOWGLIDE, must dominate ONSET. That is, pressure to satisfy ONSET generally causes glide formation but cannot cause the formation of a glide corresponding to [a] (represented as [a]). Another way to avoid an onsetless syllable would be to epenthesize an onset consonant, but the data in (35) above show that this does not occur. Since no consonant is epenthesized to allow satisfaction of ONSET, then DEP-SEG must also dominate ONSET. The combined ranking for the Dutch examples seen so far is therefore $\left\{{ }^{*}\right.$ LOWGLIDE, DEP-SEG $\}>$ ONSET $\gg>$ INTEGRITY, exemplified in (36).

No glide formation after [a]

\begin{tabular}{|r||c|c|c|c|}
\hline$/ f_{1} a_{2} r_{3} a_{4} o_{5} /$ & $*$ LOWGLIDE & $\begin{array}{c}\text { DEP- } \\
\text { SEG }\end{array}$ & ONSET & INTEG \\
\hline \hline a. fá.ra $a_{4} \cdot 0$ & & & $*$ & \\
\hline b. fá. $r a_{4} \cdot r_{6} 0$ & & $* !$ & & \\
\hline c. fá.ra $a_{4} \cdot a_{4} 0$ & $* !$ & & & $*$ \\
\hline
\end{tabular}

To summarize the important constraint interactions in (36), onsetless syllables are tolerated following [a] despite the fact that INTEGRITY is ranked lower than ONSET because an INTEGRITY violation in this case would also entail a violation of the higher-ranked *LOWGLIDE (36c). ${ }^{14}$

14. Two other constraints must also be ranked above ONSET. One is IDENT[low], which rules out a candidate such as $*\left[\right.$ fa.rá $\left.{ }_{2} \mathrm{j}_{2} \mathrm{O}\right]$ in which the input $/ \mathrm{a}_{2} /$ corresponds to some nonlow glide. The other is a constraint against the formation of an onset glide from $V_{2}$ instead of $V_{1}$; 
The outcome of [a]- $V_{2}$ hiatus is different, however, when the would-be onsetless syllable is stressed. In this case only, epenthesis of an onset consonant, [?], does occur (37).

[a]- $\mathrm{V}_{2}$ hiatus, where $\mathrm{V}_{2}$ is stressed (Booij 1995, (22))

paella [pa?ćlja] 'paella'

aorta [a?órta] 'aorta'

Kaunda [ka?únda] 'Kaunda'

These examples show that some constraint compelling stressed syllables to have onsets outranks DEP-SEG, even though DEP-SEG outranks ordinary ONSET.

The high-ranking constraint in question is ONSET/ó (38), a positional version of the augmentation constraint ONSET that is specific to stressed syllables.

$$
\begin{aligned}
\text { ONSET/o } & \text { For all syllables } x, \text { if } x \text { is a } \sigma \text {, then } a \neq b \\
\text { where } & a \text { is the leftmost segment dominated by } x \\
& b \text { is the head of } x
\end{aligned}
$$

As noted above, this constraint must dominate DEP-SEG. (The constraint *LOWGLIDE must also dominate DEP-SEG, because epenthesis is chosen instead of [a]-glide formation to provide onsets in (37).)

\begin{tabular}{|c|c|c|c|c|c|}
\hline$/ p_{1} a_{2} \varepsilon_{3} l_{4} j_{5} a_{6} /$ & *ONS/ó & $\begin{array}{c}{ }^{*} \text { Low } \\
\text { GLI }\end{array}$ & $\begin{array}{l}\text { DEP- } \\
\text { SEG }\end{array}$ & ONS & INTEG \\
\hline a. $p a_{2} . \varepsilon ́ l j a$ & *! & & 4 & * & \\
\hline b. $\quad \mathrm{pa}_{2} \cdot ?_{7} \varepsilon \dot{l} \mathrm{ja}$ & & & * & & \\
\hline c. $\quad \mathrm{pa}_{2} . \mathrm{ă}_{2} \varepsilon \dot{l j a}$ & & $* !$ & & & $*$ \\
\hline
\end{tabular}

$$
\text { Onset epenthesis in stressed syllables, after [a] }
$$

The final ranking for Dutch is therefore as shown in (40).

$$
\{\text { ONSET/ó, *LOWGLIDE }\}>>\text { DEP-SEG }>>\text { ONSET }>>\text { INTEGRITY }
$$


This ranking makes the following predictions, which match the phenomena that Booij (1995) describes: The splitting of input vowels to create glides will occur when needed to provide onsets (ONSET > INTEGRITY), except when the vowel is [a] ( ${ }^{*}$ LOWGLIDE $>$ ONSET). An underlyingly onsetless vowel following [a] will remain onsetless (DEP-SEG >> ONSET) unless that vowel is in the stressed syllable, in which case an onset consonant is epenthesized (ONSET/ó > DEP-SEG).

It might seem possible to consider an alternative analysis for Dutch [?]epenthesis in stressed syllables, under which it is a general constraint (such as ONSET) that drives [?]-epenthesis, not a position-specific constraint like ONSET/ó, and it is the blocking effect of some higher-ranked constraint $\boldsymbol{C}$ that prevents glottal stop onsets from appearing in unstressed syllables. ${ }^{15}$ Under this approach, the blocking constraint $\boldsymbol{C}$ is highest ranked; also, ONSET must dominate DEP-SEG, since epenthesis is now a "general" repair blocked in unstressed syllables, rather than a "special" repair triggered by an M/ó constraint. Because glide formation is preferred over [?]-epenthesis after vowels other than [a], the ranking DEP-SEG >> INTEGRITY is still necessary.

(41) An alternative: General [?]-epenthesis, blocked in unstressed syllables

(a) Epenthesis in stressed syllables

\begin{tabular}{|c|c|c|c|c|}
\hline /paélja/ & $\boldsymbol{C}$ & ONSET & DEP-SEG & INTEGRITY \\
\hline \hline a. pa.élja & & $* !$ & & \\
\hline b. pa.rélja & & & $*$ & \\
\hline
\end{tabular}

(b) Blocking of epenthesis in unstressed syllables

\begin{tabular}{|r|c|c|c|c|}
\hline /fárao/ & $\boldsymbol{C}$ & ONSET & DEP-SEG & INTEGRITY \\
\hline \hline \multirow{2}{*}{ a. fá.ra.o } & & $*$ & & \\
\hline b. fá.ra.?o & $* !$ & & $*$ & \\
\hline
\end{tabular}

For this alternative to work, it is necessary to find a plausible constraint to stand in for $\boldsymbol{C}$. It must be a constraint that penalizes glottal stop specifically in 
unstressed syllables (because a form with a glottal stop onset in the stressed syllable, such as [pa?źlja], can surface and so evidently satisfies $\boldsymbol{C}$ ). However, such a constraint could only take the form of a markedness constraint specific to the position unstressed syllable, which is a weak position. ${ }^{16}$ As discussed in $\S 1.3 .2$, it is preferable to allow constraints to make specific reference to phonological positions only if they are members of the set of strong positions. This alternative account for Dutch, under which the positional augmentation constraint ONSET/o is unnecessary, is viable only if constraints are permitted to make reference, not only to strong positions, but to weak positions as well. Since positional augmentation constraints-including positional ONSET constraints like ONSET/ $\sigma_{1}(\$ 4.2 .1 .1)$-are needed for the analysis of other languages, it is better to reject the $C$ analysis and account for the distribution of [?]-epenthesis in Dutch by means of ONSET/ó.

\subsubsection{ONSET/ó in Western Arrernte: Stress attraction to syllables with onsets}

The Arandic language Western Arrernte [Aranda], as described by Strehlow (1942; see Downing 1998, Breen \& Pensalfini 1999, and Pensalfini 1998 for recent analyses), assigns main stress to the initial syllable of the word unless that syllable has no onset. If the initial syllable is onsetless, stress is assigned to the following (peninitial) syllable.

(42) Stress placement in Western Arrernte

(Downing 1998:35; data from Strehlow 1942)

(a) Initial stress in C-initial words

$\begin{array}{llll}\text { párpa } & \text { 'quickly' } & \text { kála } & \text { 'already' } \\ \text { rá:tama } & \text { 'to emerge' } & \text { kútungula } & \text { 'ceremonial assistant' } \\ \text { wóratara } & \text { (place name) } & & \end{array}$

16. As usual, where phonological requirements are concerned, a (positional) faithfulnessbased analysis is not a viable option. The ranking FAITH(?)/ó $\gg>$ *? $\gg$ FAITH(?) can account for the restriction of glottal stop to stressed syllables only, but does not explain why glottalstop epenthesis is obligatory for an otherwise vowel-initial stressed syllable. 
(b) Second-syllable stress in V-initial words $(>2 \sigma)$

$\begin{array}{ll}\text { ergúma } & \text { 'to seize' } \\ \text { ulúrba } & \text { 'cold; cold wind' } \\ \text { urká:buma } & \text { 'to work' }\end{array}$

The Arrernte pattern is the stress attraction counterpart to the stressed-syllable alteration pattern seen in Dutch. In both languages, stress must fall on a syllable that has an onset, because the positional augmentation constraint ONSET/ó is high ranking. In Dutch, this is accomplished through the epenthesis of an onset for the stressed syllable, violating the faithfulness constraint DEP-SEG. In Western Arrernte, there is no epenthesis or other faithfulness violation. Instead, the constraint that requires initial stress, ALIGN-L(ó, PrWd), is (minimally) violated, so that stress can fall on a syllable that already has an onset.
ALIGN-L(ó, PrWd)
$\forall \sigma^{\prime} \exists \operatorname{PrWd}$ such that the Left edges of $\sigma^{\prime}$ and PrWd are aligned (McCarthy \& Prince 1993a) ('Stress is leftmost in the prosodic word')

The fact that Western Arrernte chooses to satisfy ONSET/o by disrupting the location of the stress, rather than epenthesizing an onset or deleting the initial vowel, shows that DEP-SEG and MAX-SEG dominate ALIGN-L(ó, PrWd). ONSET/ó must also dominate ALIGN-L(ó, PrWd), or the stressed-syllable onset requirement would not be enforced at the expense of alignment.

ONSET/ó satisfaction through misalignment

\begin{tabular}{|r||c|c|c|c|}
\hline /ulurba/ & ONSET/ó & MAX-SEG & DEP-SEG & $\begin{array}{c}\text { ALIGN-L } \\
\text { (ó, PrWd) }\end{array}$ \\
\hline \hline a. úlurba & $* !$ & & & \\
\hline b. lúrba & & $* !$ & & \\
\hline c. túlurba & & & $* !$ & \\
\hline d. ulúrba & & & & $*$ \\
\hline e. ulurbá & & & & $* * !$ \\
\hline
\end{tabular}


Thus, in Western Arrernte, the segmental composition of a vowel-initial word is unchanged, but the left-alignment of stress is compromised. As usual, however, the alignment constraint is only minimally violated; realizing the stress any further to the right than the peninitial syllable merely increases violation of ALIGN-L( $\dot{\sigma}$, PrWd) without improving performance on any higher-ranked constraint.

To give a complete analysis of Western Arrernte stress, it is necessary to consider also the placement of stress in disyllabic vowel-initial words (Arrernte has a disyllabic minimal-word requirement). In these words, stress falls on the initial, onsetless syllable.

(45) Stress in disyllabic V-initial words

(Downing 1998:35; data from Strehlow 1942)

$\begin{array}{ll}\text { á:twa 'man' } \\ \text { ílba } & \text { 'ear' }\end{array}$

Thus, the constraint NONFINALITY must dominate ONSET/ $/{ }^{17}$ As a result, it is better to stress an onsetless syllable than to have stress on the final syllable.

$$
\text { NonFinality The stressed syllable is not final in the PrWd }
$$

(Prince \& Smolensky 1993:40)

Initial stress in disyllabic words

\begin{tabular}{|r||c|c|c|}
\hline /a:twa/ & NoNFin & ONSET/ó & ALIGN-L(ó, PrWd) \\
\hline \hline a. ártwa & & $*$ & \\
\hline b. a:twá & $* !$ & & \\
\hline
\end{tabular}

Thus, the constraint ranking responsible for stress placement in Arrernte is as follows.

17. The reverse ranking, ONSET/ó $>>$ NONFINALITY, is seen in closely related Alyawarra (Yallop 1977), in which even disyllabic words avoid initial stress on an onsetless syllable. 


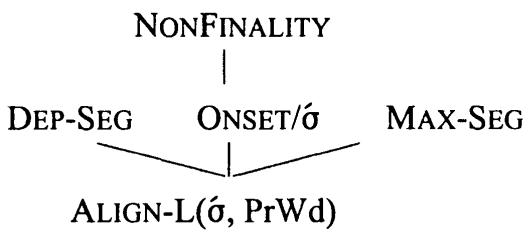

The positional augmentation constraint ONSET/o dominates the stress-location constraint ALIGN-L(ó, PrWd), which is also dominated by the faithfulness constraints MAX-SEG and DEP-SEG. Therefore, initial stress is generally sacrificed when that would mean stress on an onsetless syllable. However, since NONFINALITY dominates ONSET/o, stress is realized on an initial onsetless syllable when the only other available syllable is the word-final syllable.

In this account of Arrernte stress, the constraint ONSET/ó is crucial in driving stress away from onsetless syllables. Other analyses of Arrernte have also been developed. Downing (1998) proposes that stress avoids initial onsetless syllables because they fall outside the prosodic word. In her account, a high-ranking alignment constraint demands that the PrWd be left-aligned with a syllable that has an onset. The initial syllable is thus excluded from the PrWd in a vowel-initial (morphological) word, and stress must be realized on some syllable that is actually in the PrWd. However, the crucial PrWd-to-onsetful-syllable constraint in Downing's (1998) system is created by the Boolean conjunction (in the sense of Crowhurst \& Hewitt 1997) of two rather different constraints, ONSET and ALIGN$\mathrm{L}(\mathrm{PrWd}, \sigma)$. Downing argues that the conjunction of these two constraints is legitimate because the (left edge of the) syllable is an argument of both constraints. However, while the constituent $\sigma$ is arguably the focus - the element associated with universal quantification-of ONSET, it is not the focus of the alignment constraint (the second argument of an alignment constraint is associated with existential quantification; McCarthy \& Prince 1993a). Thus, pending further development of the theory of constraint conjunction, it remains to be seen whether ONSETกALIGN$\mathrm{L}(\mathrm{PrWd}, \sigma)$ is in fact a well-formed constraint. ${ }^{18}$

18. A related possibility, that avoids the questions raised by the use of constraint conjunction in Downing's (1998) analysis, is to propose a constraint of the form ALIGNL(PrWd, C), in the spirit of Goedemans (1996; see also Buckley 1998 for a related proposal). There appears to be no empirical distinction between ONSET/ó and a potential ALIGN-L(PrWd, C) constraint in the context of Arrernte stress. Nevertheless, the movement of stress to syllables with onsets, as seen in Arrernte, is precisely the kind of stress-attraction 
Breen \& Pensalfini (1999) have yet another take on Arrernte stress. They propose that Arrernte words are all vowel-initial at the time when stress is assigned, stress is always assigned to the second syllable of the word, and apparent consonant-initial words with initial stress are created by a later rule that deletes all word-initial schwas. However, this analysis (which reflects historical developments in Arrernte) is advanced as part of Breen and Pensalfini's proposal that all syllables in Arrernte have the structure [.VC.], and such a proposal runs counter to the widely accepted typological universal that languages with VC syllables also have $\mathrm{CV}$ and CVC syllables (Jakobson 1962). Furthermore, even if Breen and Pensalfini's diachronically based analysis of Arrernte stress proves to be the most insightful approach, there exist languages outside Arandic with the same onset-sensitive stress-placement pattern; for example, Downing (1998) mentions Banawá (citing Buller, Buller \& Everett 1993 and Everett 1995) and Iowa-Oto (citing Robinson 1975). ${ }^{19}$

In summary, Dutch and Western Arrernte both show the effects of high-ranking ONSET/ $\sigma$, a constraint that is predicted to exist because the Prominence Condition allows any $\mathbf{M} / \boldsymbol{\sigma}$ constraint that enforces the presence of some perceptually prominent property. The difference between the two languages, as for all cases of alteration versus stress-attraction phenomena, stems from whether it is a stresslocation constraint or a faithfulness constraint that is violated to satisfy the highranking positional augmentation constraint.

The next two subsections exemplify a different onset-related M/ó constraint, or rather, constraint subhierarchy: $\left[{ }^{*} \mathrm{ONSET} / \mathrm{X}\right] / \sigma$. The ${ }^{*} \mathrm{ONSET} / \mathrm{X}$ subhierarchy enforces low sonority in segments that are parsed as syllable onsets. Since lowsonority onsets contribute to the perceptual prominence of syllables, the constraints in this subhierarchy are legitimate augmentation constraints, and the positional version of the subhierarchy [ $\left.{ }^{*} \mathrm{ONSET} / \mathrm{X}\right] / \sigma$ is predicted to exist. Indeed, there are languages in which members of this subhierarchy are ranked high enough to have an effect on the composition of stressed syllables. In Niuafo'ou (\$3.2.2.3), the constraint $\left[{ }^{*}\right.$ ONSET/GLI]/ó keeps glide onsets out of stressed syllables. In Pirahã (§3.2.2.4), [ ${ }^{*}$ ONSET/D]/o, the constraint against voiced obstruent onsets in stressed syllables, forces stress to be attracted to syllables with voiceless obstruent onsets.

effect that is predicted by the existence of the constraint ONSET/ó, which is independently motivated in the analysis of Dutch given above.

19. In a paper written after Breen \& Pensalfini (1999), Pensalfini (1998) presents an OT analysis of Arrernte word-edge phenomena in which the assumption that all syllables are .VC. is no longer necessary. 


\subsubsection{3 [ ${ }^{*}$ ONSET/X]/ó in Niuafo'ou: Avoidance of glide onsets in stressed syllables}

In Niuafo'ou (Polynesian; Tsukamoto 1988, de Lacy 2000, 2001), although glide onsets are not found in native forms, they do occur in loanwords (49).

(49) Glide onsets in Niuafo'ou

(de Lacy 2000, to appear; data from Tsukamoto 1988)

$\begin{array}{ll}\text { ju.ní.tị } & \text { 'unit' } \\ \text { wa.é.a } & \text { 'wire' } \\ \text { we.lì.na.tó.nị } & \text { 'Wellington' } \\ \text { wa.í.ne } & \text { 'wine' }\end{array}$

But there is an absolute ban on glide onsets in main-stress syllables-they are always avoided, even in loanwords (50). Loanwords that have glide onsets to stressed syllables in the source language are realized in Niuafo'ou with a fully syllabic high vowel preceding the stressed syllable.

(50) No glide onsets in stressed syllables (de Lacy 2000, 2001; data from Tsukamoto 1988)

$\begin{array}{lll}\text { i.á.te } & \text { *já.te } & \text { 'yard' } \\ \text { u.á.fy } & \text { *wá.fy } & \text { 'wharf' } \\ \text { u.i.pi } & \text { *wí.pi } & \text { 'whip' }\end{array}$

In order to account for the special prohibition against glides in the onsets of stressed syllables, de Lacy (2000) argues for the existence of the constraint subhierarchy [ ${ }^{*}$ MARGIN/X]/o, with [ ${ }^{*}$ MARGIN/GLI]/ó ranked high in Niuafo'ou. As discussed in \$2.3.2.3.3, the "margin" constraint subhierarchy that is responsible for onset-sonority effects is implemented here as ${ }^{*}$ ONSET/X, formulated as in (51) for the anti-glide step of the subhierarchy and relativized to the position stressed syllable.

[*ONSET/GLI]/o For every segment $a$ that is the leftmost pre-moraic segment of some syllable $x$, if $x$ is a ó, then $|a|<$ GLI

where $|y|$ is the sonority of segment $y$ 
This positional augmentation constraint is undominated in Niuafo'ou, since nothing can ever force glide onsets to surface in stressed syllables. In particular, [ ${ }^{*}$ ONSET/GLI]/ó must dominate ONSET and ONSET/ó, because stressed syllables avoid glide onsets by appearing with no onset at all. [ ${ }^{*}$ ONSET/GLI]/o must also dominate the faithfulness constraint that regulates moraic status (informally, "FAITH $(\mu) ")$, because even if a form had an underlying glide, as in the potential input /jate/, it would surface as a high vowel rather than as an onset to the stressed syllable. $^{20}$

Avoidance of glide onsets in stressed syllables

\begin{tabular}{|r||c|c|c|c|}
\hline /jate/ & {$\left[{ }^{*}\right.$ ONSET/GLI]/ó } & ONSET/ó & ONSET & FAITH $(\mu)$ \\
\hline \hline a. já.te & $* !$ & & & \\
\hline b. i.á.te & & $*$ & $*$ & \\
\hline
\end{tabular}

The ranking ONSET $>$ FAITH $(\mu)$ is motivated, as de Lacy (2000, to appear) argues, because high vocoids that appear before non-main stress vowels always surface as glide onsets, never as vowels (53).

Glide onsets in other syllables

\begin{tabular}{|c|c|c|c|c|}
\hline /iuniti/ & $\begin{array}{c}\text { ["ONSET/ } \\
\text { GLI]/ó }\end{array}$ & ONSET/ó & ONSET & $\operatorname{FAITH}(\mu)$ \\
\hline a. i.u.ní.ti & & & *! & \\
\hline b. ju.ní.ti & & & & $*$ \\
\hline
\end{tabular}

Of course, as seen in (52), the positional augmentation constraint $\left[{ }^{*} \mathrm{ONSET} / \mathrm{GLI}\right] / \boldsymbol{\sigma}$ outranks ONSET, but since the glide onset in (53) above is not part of the stressed syllable, $\left[{ }^{*} \mathrm{ONSET} / \mathrm{GLI}\right] / \sigma$ is irrelevant for this form.

20. de Lacy (2000) assumes that a potential input like /jate/ will surface as [i.a.te] rather than as, e.g., [a.te] or [ta.te] - that is, that vocalization of the glide is the chosen repair. There are apparently no overt alternations to show that this is in fact so, but since the (English) source words for the forms in question do have glide onsets in the stressed syllables, and these correspond to high vowels in Niuafo'ou, it seems plausible that glide vocalization is in fact the chosen repair for an impossible form like /jate/. 
Thus, the fact that glide onsets are prohibited from stressed syllables, but are in fact preferred to vowels in hiatus in other syllables, is evidence for a stressed syllable-specific version of the augmentation constraint *ONSET/GLI, namely, [*ONSET/GLI]/ó.

\$2.3.2.3.3 argues that ONSET is not simply "[*ONSET/Ø]," an endpoint of the *ONSET/X subhierarchy, but must be viewed as a formally separate constraint. The crucial ranking [*ONSET/GLI]/o >> ONSET/ó in Niuafo'ou is evidence for this claim. Since ONSET/ó must be ranked below [ ${ }^{*}$ ONSET/GLI]/o, ONSET/ó cannot possibly be the same thing as "[*ONSET/Ø]/o," which would be universally ranked at the top of the $\left[{ }^{*} \mathrm{ONSET} / \mathrm{X}\right] / \sigma$ hierarchy. (See also $\S 4.2 .1 .2 .5$ on differences between ONSET and ${ }^{*}$ ONSET/X.)

\subsubsection{4 $\left[{ }^{*}\right.$ ONSET/X]/ó in Pirahã: Stress attraction and low-sonority onsets}

Once again, there is a close link between stressed-syllable alteration and the attraction of stress to syllables that have particular properties. In Niuafo'ou, considered above, the ranking of the $\left[{ }^{*} \mathrm{ONSET} / \mathrm{X}\right] / \sigma$ hierarchy causes a syllable that would otherwise surface with a glide onset to be altered so that the glide onset is not present in the output form when the syllable is stressed. Stress-attraction phenomena driven by [ $\left.{ }^{*} \mathrm{ONSET} / \mathrm{X}\right] / \sigma$ constraints are also observed, as for example in Pirahã (an Amazon language of the Mura family; Everett \& Everett 1984ab; Everett 1988), where stress is attracted to the syllable with the lowest-sonority onset available. (Other positional augmentation constraints are also active in Pirahã: ONSET/ó and HEAvYo/ó.)

While Pirahã is a tone language, contrasting high and low tones, it also has phonologically relevant stress that is independent of tone. (In all Pirahã data shown here, stress is indicated with an acute accent, and high tone is marked by underlining. Vowels that are not underlined have low tones.)

Tone does not affect stress placement (Everett \& Everett 1984a)

$\begin{array}{llll}\text { Páo.oi } & \text { 'foreigner' } & \text { Páo.ọi } & \text { 'ear' } \\ \text { Páo.oị } & \text { 'type of fruit' } & \text { Páo.oi } & \text { 'skin' } \\ \text { Páo.oi } & \text { 'Brazil nut shell' } & & \end{array}$

Everett (1988) argues that stress in Pirahã has phonological relevance. For example, native speakers correct mistakes in stress placement made by non-native speakers, 
and there are optional processes of devoicing and deletion that are sensitive to stress placement.

In Pirahã (as in other languages such as Spanish; Harris 1983), there is a "window" for stress: it must fall within the last three syllables. However, the determination of stress placement within that window is dependent on the characteristics of the last three syllables. Long vowels and diphthongs take precedence over short vowels (there are no CVC syllables), and if two syllables have the same rime weight, then syllables with voiceless onsets are preferred to those with voiced onsets, which in turn are preferred to onsetless syllables. (In the examples below, the three classes of onsets are labeled T, D, and $\emptyset$ respectively.)

The data in (55) show that, regardless of onset class, stress is placed on a heavy syllable (CVV) rather than on a light one (CV).

Stress is attracted to heavy syllables (Everett \& Everett 1984a)

$\begin{array}{lll}\varnothing \mathrm{VV}>\mathrm{TV} & \text { ho.ái..pi } & \text { 'type of fish' } \\ \varnothing \mathrm{VV}>\mathrm{TV}, \mathrm{DV} & \text { ?a.ho.áo.gi } & \text { (proper name) } \\ \mathrm{DVV}>\mathrm{TV} & \text { bíi.si } & \text { 'red' } \\ \mathrm{DVV}>\mathrm{TV}, \mathrm{DV} & \text { gíi.so.gi } & \text { 'turtle' }\end{array}$

The attraction of stress to heavy syllables can be accounted for by HEAvYo/o, the positional augmentation constraint that requires stressed syllables to be heavy (§3.2.1.1). Since onset-sonority effects appear only when rime weight is held constant, HEAVYo/ó must outrank the constraints that are sensitive to onset sonority. However, the matter of syllable weight will be set aside for now, and only syllables with equivalent rime weight will be compared, because the focus of the current discussion is onset sonority.

The scale of onset preference, $\mathrm{T} \succ \mathrm{D} \succ \varnothing$, is demonstrated in (56).

Onset-sensitive stress placement (Everett \& Everett 1984a)

(Italics indicate the relevant syllables to be compared.)

(a) Heavy syllables

$\begin{array}{cll}\text { TVV > DVV } & \text { káa.gai } & \text { 'word' } \\ & \text { pa.hái.bii } & \text { (proper name) } \\ \text { biï.sái } & \text { 'red' } \\ & \text { ii.baọ.sái } & \text { 'her cloth' }\end{array}$




\begin{tabular}{|c|c|c|}
\hline TVV $>\varnothing V V$ & $\begin{array}{l}\text { poo.hói.hị.ai } \\
\text { ?ị.sií.ho.ại } \\
\text { soi.oạ.ga.hái } \\
\text { ko.so.ii.gai.tái }\end{array}$ & $\begin{array}{l}\text { 'fish' } \\
\text { 'liquid fuel' } \\
\text { 'thread' } \\
\text { 'eyebrow' }\end{array}$ \\
\hline $\mathrm{DVV}>\varnothing \mathrm{VV}$ & $\begin{array}{l}\text { gáo.ii } \\
\text { poo.gái.hi.aí } \\
\text { gi.ai.bái }\end{array}$ & $\begin{array}{l}\text { (proper name) } \\
\text { 'banana' } \\
\text { 'dog' }\end{array}$ \\
\hline
\end{tabular}

(b) Light syllables ${ }^{21}$

$\begin{array}{lll}\text { TV }>\text { DV } & \text { 7á.ba.gi } & \text { 'toucan' } \\ & \text { ti.pó.gi } & \text { 'species of bird' } \\ \text { ka.gi.hí } & \text { 'wasp' } \\ \text { ?a.ba.pá } & \text { (proper name) }\end{array}$

Thus, syllables with voiceless obstruent onsets are preferred to those with voiced onsets, which in turn are preferred to syllables with no onset at all. The difference between voiced and voiceless obstruents can be viewed as a difference in sonority (see $\S 2.3 .2 .2$ for discussion). Since the ${ }^{*}$ ONSET/X subhierarchy is derived from the segmental sonority scale, this means that there are *ONSET/X constraints that distinguish between voiced $(D)$ and voiceless $(T)$ obstruents. For Pirahã, it is the positional versions of these constraints, relativized to stressed syllables, that are relevant.

$\left[{ }^{*} \mathrm{ONSET} / \mathrm{D}\right] / \mathbf{\sigma}$

$\left[{ }^{*} \mathrm{ONSET} / \mathrm{T}\right] / \sigma$
For every segment $a$ that is the leftmost pre-moraic segment of some syllable $x$, if $x$ is a ó, then $|a|<\mathrm{D}$

For every segment $a$ that is the leftmost pre-moraic segment of some syllable $x$, if $x$ is a ó, then $|a|<\mathrm{T}$

These two constraints, being members of the $\left[{ }^{*} \mathrm{ONSET} / \mathrm{X}\right] / \sigma$ subhierarchy, are universally ranked: $\left[{ }^{*}\right.$ ONSET/D $] / \sigma o ~>~\left[{ }^{*}\right.$ ONSET/T $] / \sigma$. With $\left[{ }^{*}\right.$ ONSET/D $] / \sigma$ ranked above the constraint that would otherwise determine default stress placement, and with faithfulness to voicing undominated, then syllables with voiceless onsets will be preferred to those with voiced onsets for stress. 
The constraint that is responsible for default stress placement can be determined by the stress pattern of words with syllables that agree in rime weight and onset sonority. In such cases, there is a preference for stress on the rightmost syllable.

$$
\text { All else being equal, stress is rightmost }
$$

(Everett \& Everett 1984a, Everett 1988)

TVV TVV 'TVV

(DVV) TVV 'TVV

TV TV 'TV

DV DV 'DV

\section{pao.hoa.hái} bai.tọi.sái ko.?o.pá gị.go.gí 'anaconda'

'wildcat'

'stomach'

'what about you?'

The preference for right-edge stress can be seen as the result of the constraint AlIGN-R(o, PrWd). This constraint is dominated by the stressed-syllable onsetsonority constraint [*ONSET/D]/ó (and by HEAVYo/ó), since rightmost stress emerges only when onset sonority (and rime weight) are equal across syllables.

(59) Avoiding voiced onsets has priority over right-edge stress

(a) Default stress is rightmost

\begin{tabular}{|c|c|c|c|}
\hline /paohoahai/ & $\begin{array}{c}{[* \text { ONSET/ }} \\
\text { D]/ó }\end{array}$ & $\begin{array}{c}{\left[{ }^{*} \text { ONSET/ }\right.} \\
\text { T]/ó }\end{array}$ & $\begin{array}{c}\text { ALIGN-R } \\
(\dot{\sigma}, \operatorname{PrWd})\end{array}$ \\
\hline a. páo.hoa.hai & & $*$ & $* ! *$ \\
\hline b. pao.hóa.hai & & $*$ & $* !$ \\
\hline c. pao.hoa.hái & & $*$ & \\
\hline
\end{tabular}


(b) Voiceless onsets preferred to voiced

\begin{tabular}{|c||c|c|c|}
\hline /7abagi/ & $\begin{array}{c}\text { [*ONSET/ } \\
\text { D]/ó }\end{array}$ & $\begin{array}{c}{\left[{ }^{*} \text { ONSET/ }\right.} \\
\text { T]/óo }\end{array}$ & $\begin{array}{c}\text { ALIGN-R } \\
\text { (o, PrWd) }\end{array}$ \\
\hline \hline a. 7á.ba.gi & & $*$ & $* *$ \\
\hline b. 7a.bá.gi & $* !$ & & $*$ \\
\hline c. 7a.ba.gí & $* !$ & & \\
\hline
\end{tabular}

As noted above, however, syllables with voiced onsets are preferred to syllables with no onsets at all. This means that ONSET/o dominates [*ONSET/D]/ó. However, ONSET/o is itself dominated by the constraint or constraints responsible for keeping the stress within the final three syllables, encapsulated here as "STRESSWINDOW"; thus, every word has stress within that three-syllable window. (See Green (1995) for one proposal concerning the nature of the constraints enforcing a three-syllable stress window in Pirahã.)

(60) Even voiced onsets are preferred to onsetless syllables ${ }^{22}$

\begin{tabular}{|r|c|c|c|c|c|}
\hline /poo.gai.hi.ai] & $\begin{array}{c}\text { STRS } \\
\text { WIN- } \\
\text { DOW }\end{array}$ & $\begin{array}{c}\text { ONS } \\
/ \sigma\end{array}$ & $\begin{array}{c}{\left[{ }^{*} \mathrm{ONS} /\right.} \\
\mathrm{D}] / \sigma\end{array}$ & $\begin{array}{c}{\left[{ }^{*} \mathrm{ONS} /\right.} \\
\mathrm{T}] / \sigma\end{array}$ & $\begin{array}{c}\text { AL-R } \\
(\dot{\sigma}, \\
\text { PrWd }\end{array}$ \\
\hline \hline a. poo.gái.hi.ai & & & $*$ & & $* *$ \\
\hline b. poo.gai.hi.ái & & $* !$ & & & \\
\hline c. póo.gaai.hi.ai & $* !$ & & & $*$ & $* * *$ \\
\hline
\end{tabular}

Thus, the constraint ranking motivated here for Pirahã is as in (61).

(61) Constraint ranking for Pirahã stress

$$
\begin{aligned}
& \text { STRESSWINDOW }>>\text { HEAVY } / \text { /o }>>\text { ONSET/ó }>>\left[{ }^{*} \text { ONSET/D }\right] / \sigma o> \\
& \left\{\left[{ }^{*} \mathrm{ONS} / \mathrm{T}\right] / \sigma, \operatorname{ALIGN}-\mathrm{R}(\dot{\sigma}, \operatorname{PrWd})\right\}
\end{aligned}
$$

22. An output candidate with penultimate stress, [poo.gai.hí.ai], is not considered here because, as noted above, rime weight takes precedence over onset sonority. 
The ranking relationship of greatest interest here is $\left[{ }^{*}\right.$ ONSET/D $] / \sigma>$ ALIGN$\mathrm{R}(\dot{\sigma}$, PrWd), which accounts for the ability of the onset-sonority preference (voiceless over voiced) to override default right-edge stress (as long as higherranked constraints like STRESSWINDOW and HEAVYo/ó are satisfied). The constraints of the *ONSET/X subhierarchy, and therefore of its positional versions like $\left[{ }^{*} \mathrm{ONSET} / \mathrm{X}\right] / \sigma$, are in a universally fixed ranking derived from the segmental sonority scale. Consequently, the fact that $\left[{ }^{*} \mathrm{ONSET} / \mathrm{D}\right] / \sigma$ is active in Pirahã entails that the higher-ranking members of the subhierarchy, $\left[{ }^{*}\right.$ ONSET/LOWV]/o $>>$

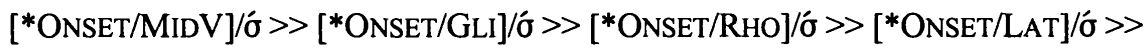
$\left[{ }^{*}\right.$ ONSET/NAS]/o, are also ranked high enough to be active. However, the effect of these constraints cannot be seen, as they are vacuously satisfied-Pirahã has no such segments in its phonemic inventory.

$$
\text { Segmental phoneme inventory of Pirahã (Everett \& Everett 1984a) }{ }^{23}
$$

$$
\text { /pt (k) ? shbgiao/ }
$$

Interestingly, it may be the general version of the *ONSET/X subhierarchy that is responsible for such a segmental inventory. Pirahã has no closed syllables, so all consonants are onsets. If the constraints *ONSET/GLI > ${ }^{*}$ ONSET/RHO $>$ *ONSET/LAT $\gg *$ ONSET/NAS are ranked above MAX-SEG or IDENT[f], this would explain the absence of any consonant higher in sonority than the voiced obstruents. $^{24}$

\subsubsection{Concluding remarks on stress/onset interactions}

The last four languages discussed-Dutch, Western Arrernte, Niuafo'ou, and Pirahã-show that there are interactions between stressed syllables and onsetrelated properties that pattern just like the familiar stress/prominence interactions

23. Everett \& Everett (1984b) state that [k] appears to be a variant of [h], as well as a portmanteau of the sequence [hi], and is probably not an independent phoneme.

24. It is apparently not the case that Pirahã completely lacks sonorant consonants in surface forms. Everett (1988:106) states that /b/ and /g/ have optional realizations as nasals (following pause or word-initially) and as "vibrants" (in certain intervocalic environments). The analysis outlined here predicts that these phonemes are most likely to be realized as stops when they are in the main-stress syllable, although since it is not clear which constraints drive their (optional) realization as sonorants, it is impossible to predict exactly what the pattern would be. 
reviewed in \$3.2.1. Namely, there are languages in which stressed syllables are altered to have onsets or to have low-sonority onsets, and there are languages in which stress is attracted to syllables that have onsets or to syllables that have lowsonority onsets.

The existence and the nature of these stress/onset interactions follow directly from the theory of $\mathbf{M} / \mathbf{s t r}$ constraints developed in this dissertation. First, stressedsyllable versions of ONSET and the *ONSET/X subhierarchy exist because these constraints are prominence-enhancing, satisfying the Prominence Condition. Second, these $\mathbf{M} / \mathbf{\sigma}$ constraints, like the $\mathbf{M} / \mathbf{\sigma}$ constraints in $\S 3.2 .1$, are able to give rise to either alteration or attraction effects because the difference between the two patterns depends on the relative ranking among other constraints in the system (faithfulness constraints related to the prominent property in question versus stresslocation constraints).

This approach to onset-related stress-attraction effects also makes a contribution to the long-standing debate about the role of syllable onsets in stress assignment. Syllable weight is determined without reference to onsets; this is one of the insights that has led to the development of moraic theory (Hyman 1985; Hayes 1989), in which onsets are not weight-bearing elements. Nevertheless, several cases of onset-sensitive stress placement have been reported (see, e.g., Davis 1988). As should be obvious from the nature of the stressed-syllable augmentation constraints introduced throughout this section, the approach taken here is consistent with an insight from Hayes (1995:Ch 7): not every property that is involved in stress placement need be related directly to syllable weight in the narrow sense of mora count, since other dimensions of prominence (including tone and vowel quality as well as properties of syllable onsets) may also be independently invoked. Thus, it seems reasonable to separate onset-related stress effects from syllable weight per se. (Recall that in Pirahã, syllable weight in the conventional sense has absolute priority over onset preferences in stress placement; this fact further supports the claim that these two factors affecting stress placement are distinct rather than cumulative.)

It follows from the theory developed here that stress placement can be sensitive to some property of syllable onsets only if that property involves perceptual prominence. This is because stress attraction effects are a response to demands imposed by high-ranking stressed-syllable augmentation constraints, and the only possible positional augmentation constraints are those involving properties that serve to enhance perceptual prominence.

The prediction that onset-sensitive stress placement is necessarily related to perceptually prominent properties appears to be borne out. The languages surveyed by Davis (1988), in which stress is sensitive to certain characteristics of syllable 
onsets, include Pirahã and Western Arrernte, which, as shown above, do involve independently attested stressed-syllable augmentation constraints.

Another case that Davis (1988) also discusses is the Australian language Mathimathi [Madimadi], based on work by Hercus (1969). Hercus and Davis propose that stress placement in this language is sensitive to whether or not an onset consonant is coronal: according to Davis's analysis, stress is attracted away from an initial light syllable onto the second syllable if the onset of the second syllable is coronal. If stress placement in Mathimathi truly were sensitive to the place of articulation of onset consonants, then this would be a counterexample to the claim that onset-sensitive stress must involve prominence-enhancing constraints-except in the case of laryngeal versus supralaryngeal places of articulation (see $\S 3.4$ below), there does not seem to be a phonologically relevant distinction in prominence among the different places of articulation.

However, the stress pattern of Mathimathi has been reanalyzed by Gahl (1996), who shows that stress is assigned on the basis of morphological, rather than phonological, factors. That is, stress falls on the final syllable of the morphological stem. The reason for the apparent relationship of coronal consonants to stress is that possible root shapes in Mathimathi are quite restricted. Out of 104 roots of the form CVVㄷ and 22 of the form CVㄷV in Hercus (1969), every single one has a coronal consonant in the intervocalic position. Therefore, disyllabic roots always have second-syllable stress, and they always have coronal onsets in the second syllable, but stress placement is truly determined with reference to the right edge of the stem rather than to place of articulation in onset consonants. Gahl supports this alternative analysis by analyzing a number of forms that would be exceptions to a coronal-onset account of stress placement but are consistent with the morphological account.

Davis (1988) discusses two further examples of onset-sensitive stress assignment. The first involves -ere verbs in Italian (based on work by Davis, Manganaro, \& Napoli 1987), which according to his analysis attract stress onto the antepenultimate syllable (away from the penult), as in lú.ce.re 'to be light,' if the root final vowel has a sonorant onset (among other factors). The second is stress assignment in -ative adjectives in English, in which the first syllable of the suffix is destressed when it has a sonorant onset (as in nóminative, *nóminàtive; cf. quálitàtive). The crucial onset property in both these cases is sonority, which is a property that is relevant to perceptual prominence, although the Italian pattern seems to go the wrong way (with stress being attracted to a syllable that has a relatively poor sonority contrast between onset and nucleus). In any case, as noted by Gahl (1996:343), these two examples both involve morphologically restricted sets of words; it is not necessarily clear that a phonological account of stress placement in these cases is the correct approach. 
Thus, it seems to be the case that phonological, productive instances of onsetsensitive stress placement do involve perceptually prominent properties, as predicted by the positional augmentation approach to stress-attraction effects.

\subsubsection{Summary: stressed-syllable augmentation}

This section has considered a number of languages in which stressed-syllable augmentation constraints are phonologically active. As predicted by the theory of $\mathbf{M} / \mathbf{s t r}$ constraints developed in Chapter 2, the kinds of phonological requirements that can hold specifically of stressed syllables are varied in nature, but they all involve perceptually prominent properties. The Prominence Condition ensures that all $\mathbf{M} / \mathbf{s t r}$ constraints are prominence-enhancing, and since there is no additional constraint filter on $\mathbf{M} / \Phi$ str constraints, the full set of augmentation constraints can be freely relativized to phonetically strong positions like the stressed syllable. Moreover, there are many augmentation constraints whose constraint focus is compatible with relativization to a syllable-sized position like the stressed syllable.

It has also been shown in this section that $\mathbf{M} / \mathbf{\sigma}$ constraints can account for both alteration and attraction effects in stress systems, explaining why these two types of patterns are sensitive to the same set of prominent properties.

The remaining two sections of this chapter discuss augmentation effects for two more phonetically strong positions, long vowels (§3.3) and onsets (§3.4).

\subsection{Positional augmentation in long vowels}

Another member of the set of phonetically strong positions is the long vowel. Thus, any markedness constraint that enforces the presence of perceptually prominent properties in vowels is predicted to have a counterpart that is relativized to long vowels.

The *PEAK/X subhierarchy, which calls for high sonority in vowels, qualifies as an augmentation constraint (\$2.3.2.2). As predicted, this constraint subhierarchy has a $[* \operatorname{PEAK} / \mathrm{X}] / \mathrm{V}$ : counterpart.

$\left[{ }^{*} \mathrm{PEAK} / \mathrm{X}\right] / \mathrm{V}: \quad$ For every segment $a$ that is the head of some syllable $x$, if $a$ is a V:, then $|a|>\mathrm{X}$

where $|y|$ is the sonority of segment $y$

$\mathrm{X}$ is a particular step on the segmental sonority scale 
The effects of the positional augmentation constraint [*PEAK/HIGHV]/V: are seen in the Yawelmani dialect of Yokuts (California; Newman 1944). Yawelmani has a process of vowel lowering that affects long high vowels, causing them to surface as mid vowels (Kuroda 1967, Kisseberth 1969, Archangeli 1984).

Yawelmani long-vowel lowering interacts in complex ways with other processes in the language, including vowel epenthesis and a height-sensitive process of rounding harmony; in fact, Yawelmani is a classic example that has been used to argue for abstract intermediate levels in phonological derivations (Kisseberth 1969). Therefore, Yawelmani vowel phonology poses a challenge for nonderivational theories like OT that do not recognize such intermediate levels. Several non-derivational analyses of the vowel alternations in Yawelmani have been put forward (Cole \& Kisseberth 1996; Sprouse 1997; McCarthy 1999; cf. Goldsmith 1993; Archangeli \& Suzuki 1997), but to present and evaluate these proposals in detail here is beyond the scope of the present discussion. Here, the question of how all the different vowel-related processes interact will be set aside to focus on just this one aspect of the phonology of Yawelmani vowels.

The lowering of long high vowels is exemplified by the forms in (64ab). Evidence that the root vowels in these forms, which appear as $[o(:)]^{25}$ on the surface, are underlyingly /u:/ comes from the behavior of these roots with respect to vowel harmony. Yawelmani [round] harmony occurs only between vowels of the same height (where [i u] are high and [a o] are low). Therefore, the unexpected occurrence of harmony in (64a) and the blocking of harmony in (64b) both indicate that the root vowel is underlyingly high, not low (compare the forms in $(64 \mathrm{~cd})$, formed from roots with underlying /o:/, where harmony applies as expected given the surface root vowel).

Lowering of /u:/

(Kenstowicz \& Kisseberth 1979:90; data from Newman 1944)

Roots with /u:/

(a) wo:?uy-hun 'falls asleep'

do:lul-hun 'climbs'

c'om-hun 'destroys'

şog-hun 'pulls out a cork'
Roots with /0:/

(c) so:nil-hin 'packs on the back' ho:tin-hin 'takes the scent' dos-hin 'reports' won-hin 'hides'

25. Long vowels in Yawelmani are regularly shortened when they appear in closed syllables. 

(b) woy?-al 'might fall asleep' doll-al 'might climb'
c'o:m-al 'might destroy' so:g-al 'might pull out the cork'

(d) sonl-ol 'might pack on the back' hotn-ol 'might take the scent' do:s-ol 'might report' wo:n-ol 'might hide'

There is no direct evidence from harmony patterns that surface [e:] corresponds to underlying [i:], since the unrounded version of the suffix vowels (and of the epenthetic vowel that appears in CVC_C roots) is the default form. But other phonological alternations in the language do provide evidence for an underlying /i:/ that surfaces as /e:/ (Kisseberth 1969). In any case, in an OT analysis that assumes richness of the base, it is necessary to account for why there is no surface contrast between [i:] and [e:], or between [u:] and [o:]; ${ }^{26}$ this point would still hold even without the evidence from harmony for /u:/. Clearly, something is forcing the neutralization of (potential) /i:/ and /e:/ to [e:], and that of /u:/ and /o:/ to [o:].

The non-derivational accounts of Yawelmani vowel phonology in Cole \& Kisseberth (1996), Sprouse (1997), and McCarthy (1999) differ considerably, but all of these proposals include a constraint that bans long high vowels from surface forms.

Constraints against long high vowels in Yawelmani

(a) LOWER V $\mu \mu \rightarrow[$ low]

Cole \& Kisseberth (1996)

(b) *VV[high] No [high] on a V linked to two $\mu[\ldots]$ Sprouse (1997)

(c) LONG/-HIGH If long, then non-high McCarthy (1999)

Cole \& Kisseberth (1996) give a substantive justification for their constraint LOWER that is entirely compatible with the notion of positional augmentation under development here.

26. Actually, [i:] and [u:] do sometimes occur, as contractions of [iw] and [uw] respectively in certain highly restricted morphological environments (Kenstowicz \& Kisseberth 1979:99); however, the analyses of Yawelmani that I have consulted tend to regard this as a limited and exceptional phenomenon. 
Lowering can be viewed as an optimizing constraint that increases the sonority of bimoraic vowels. This is a case of the strong (in terms of weight) becoming stronger (in terms of peak sonority). (Cole \& Kisseberth 1996:13; emphasis added)

That is, [*PEAK/HIGHV]/V: (or LOWER) is a legitimate augmentation constraint, because it acts to enhance the prominence of the strong position $\mathrm{V}$ :.

\subsection{Positional augmentation in syllable onsets}

Yet another phonetically strong position is the syllable onset (as noted in §2.3.3, onset here is used as an abbreviation for "the phonetically strong position onset/released consonant"). Again, because the only filter relevant for phonetically strong positions is the Prominence Condition, any prominence-enhancing markedness constraint that can be relativized to the syllable onset is predicted to have an onset-specific counterpart. One such constraint is HAVECPLACE (§2.3.2.4). Evidence for HAVECPLACE/Onset, an onset-specific version of HAVECPLACE, is found in Chamicuro (Parker 1994, 2000).

In Chamicuro, the glottal consonants [h, ?] are contrastive members of the phoneme inventory, but they occur only in coda position and are banned from appearing as syllable onsets. Parker (1994) presents data showing that the glottals $[\mathrm{h}, ?]$ in coda position contrast with each other, with vowel length, with zero, and with other consonants (although most codas in the language are in fact glottals; S. Parker, p.c.).

(66) Coda [h, ?] contrastive in Chamicuro (data from Parker 1994)
(a) me?sa 'sea lion'
meisa 'party'
meØsa 'table'
(b) ičehki 'it burns'
iče:ki 'it is abundant' 


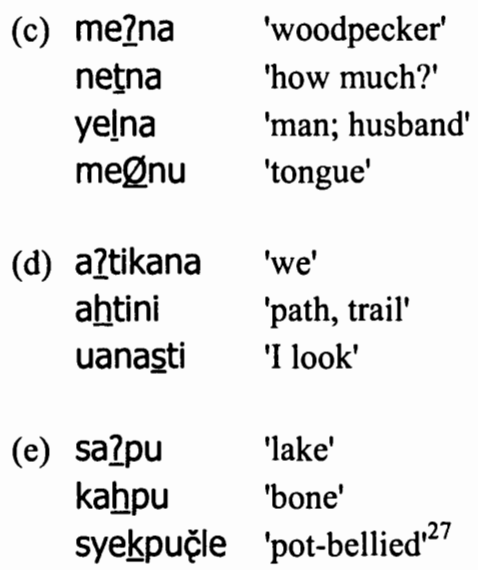

Parker $(1994,2000)$ specifically argues that [h, ?] should be considered coda consonants and not part of syllabic nuclei, because they have the same distribution as other coda consonants in the language. For example, syllables can be maximally $\mathrm{CVC}$ or CVV, and [h, ?] cannot cooccur with a long vowel or another coda consonant. Also, final syllables must be light in Chamicuro, and syllables with [h, ?] do not occur in word-final position.

Since the glottals [h, ?] are allowable segments in Chamicuro, then their inability to serve as onset consonants can obviously not be explained as part of a general prohibition against glottals in the language. Also, the familiar argument against a faithfulness-based account holds here as well; special faithfulness constraints for onsets cannot account for a language in which some permissible input structure fails to appear in onset position (see Parker 2000 for further discussion of problems that a faithfulness-based analysis would encounter). To account for the distribution of glottal consonants in Chamicuro, Parker (2000) argues that it is necessary to recognize a markedness constraint that specifically bans glottal consonants in onset position: the positional augmentation constraint HAVECPLACE/Ons (the formulation given here has been slightly modified from Parker's original constraint to make it consistent with the $\mathrm{C} /$ str schema).

HAVECPlace/Ons For all consonants $x$, if $x$ is $\mathrm{C}_{[+ \text {release] }}$, then $x$ has a supralaryngeal Place specification

27. Following Parker (1994), I use the symbol [ $\check{c}]$ to stand for a retroflex alveopalatal affricate. 
Parker (2000) proposes the following ranking for Chamicuro (some constraint names have been altered here in the interest of consistent nomenclature).

(68) Glottal-related constraint ranking for Chamicuro (Parker 2000)

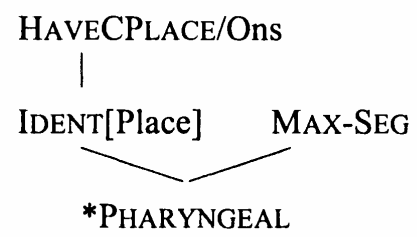

The ranking IDENT[Place], MAX-SEG $>>$ *PHARYNGEAL is necessary because outside of onset position, input glottals surface faithfully, without being either changed to a non-glottal or deleted (69a). On the other hand, HAVECPLACE/Ons must dominate at least one of the two faithfulness constraints shown in (68) above, so that an input glottal, if it is syllabified as an onset, will not surface as a glottal. According to the ranking given in Parker (2000), the crucial interaction for onset glottals is HAVECPLACE/Ons > IDENT[Place], so that an input glottal will surface as a non-glottal consonant in onset position rather than being deleted (69b).

(69) Glottal consonants surface in the coda, but not in the onset

(a) HAVECPLACE/Ons is irrelevant for coda glottals (Parker 2000); [apehta] 'sardine'

\begin{tabular}{|c||c|c|c|c|}
\hline /apehta/ & $\begin{array}{c}\text { MAX- } \\
\text { SEG }\end{array}$ & $\begin{array}{c}\text { HAVE } \\
\text { CPL/Ons }\end{array}$ & $\begin{array}{c}\text { IDENT } \\
\text { [Place] }\end{array}$ & *PHAR \\
\hline \hline a. a.peh.ta & & & & $*$ \\
\hline b. a.pe.ta & $* !$ & & & \\
\hline c. a.pek.ta & & & $* !$ & \\
\hline
\end{tabular}


(b) Onset glottals surface with a supralaryngeal Place specification (hypothetical input; Parker 2000)

\begin{tabular}{|r||c|c|c|c|}
\hline /hapeta/ & $\begin{array}{c}\text { MAX- } \\
\text { SEG }\end{array}$ & $\begin{array}{c}\text { HAVE } \\
\text { CPL/Ons }\end{array}$ & $\begin{array}{c}\text { IDENT } \\
\text { [Place] }\end{array}$ & *PHAR \\
\hline \hline a. ha.pe.ta & & $* !$ & & $*$ \\
\hline b. a.pe.ta & $* !$ & & & \\
\hline c. ka.pe.ta & & & $*$ & \\
\hline
\end{tabular}

Thus, Parker's (2000) analysis shows that an account of the distribution of glottal consonants in Chamicuro crucially depends on the existence of a constraint HAVECPlace/Ons. Parker notes that languages of the Cariban family, such as Tiriyó, restrict glottal consonants to coda position just as Chamicuro does, indicating that HAVECPLACE/Ons is high ranking in these languages as well. Under the model of M/str constraints developed here, HAVECPLACE/Ons is a well-formed positional augmentation constraint, because it requires the strong position onset to have an additional perceptually prominent property, supralaryngeal place.

\subsection{Conclusion: Predicted and attested $M / \Phi$ str constraints}

This chapter has focused on the phonetically strong positions stressed syllable, long vowel, and onset/released consonant, presenting a number of languages in which markedness constraints relativized to these positions play a crucial role.

Under the Schema/Filter model of CoN, all formally possible constraints exist unless there is a constraint filter that excludes them. The Prominence Condition is one filter that applies to $\mathbf{M} / \mathbf{s t r}$ constraints, ensuring that markedness constraints are relativized to strong positions only when they are augmentation (prominenceenhancing) constraints. If there are no other relevant filters, then there should be no further restrictions on possible $\mathbf{M} / \mathbf{s t r}$ constraints. This prediction is borne out for the phonetically strong positions, as shown in (70). Setting aside cases of domain mismatch, in which the size of the strong position is not compatible with the focus of the augmentation constraint (\$2.2.2), nearly all expected combinations of augmentation constraints and strong positions do occur. The only exceptions are constraints that are vacuously satisfied by any output candidate $\left(\left[{ }^{*} \mathrm{ONSET} / \mathrm{X}\right] / \mathrm{V}\right.$ :, HAVESTRESS/ó) or essentially indistinguishable from other constraints ([ $\left.\left.{ }^{*} \mathrm{PEAK} / \mathrm{X}\right] / \mathrm{Ons},\left[{ }^{*} \mathrm{ONSET} / \mathrm{X}\right] / \mathrm{Ons}\right)$; see $\S 2.3 .3$ for further discussion of these cases. 
(70) Predicted augmentation constraints for phonetically strong positions

\begin{tabular}{|c|c|c|c|}
\hline & ó & V: & onset \\
\hline HEAVYo & $\begin{array}{l}\text { - Mohawk } \\
\text { - West } \\
\text { Germanic } \\
\text { - Aguacatec }\end{array}$ & $\begin{array}{l}\text { domain } \\
\text { mismatch }\end{array}$ & $\begin{array}{l}\text { domain } \\
\text { mismatch }\end{array}$ \\
\hline HTONE & $\begin{array}{l}\text { - Slave } \\
\text { - Golin } \\
\text { - Serbo- } \\
\text { Croatian }\end{array}$ & $\begin{array}{l}\text { domain } \\
\text { mismatch }\end{array}$ & $\begin{array}{l}\text { domain } \\
\text { mismatch }\end{array}$ \\
\hline$\left[{ }^{*} \mathrm{PEAK} / \mathrm{X}\right]$ & $\begin{array}{l}\text { - Slovene } \\
\text { - Mordwin } \\
\text { - English }\end{array}$ & - Yawelmani & $\begin{array}{l}\text { (hard to } \\
\text { distinguish } \\
\text { from other } \\
\text { constraints) }\end{array}$ \\
\hline ONSET & $\begin{array}{l}\text { - Dutch } \\
\text { - W. Arrernte }\end{array}$ & $\begin{array}{l}\text { domain } \\
\text { mismatch }\end{array}$ & $\begin{array}{l}\text { domain } \\
\text { mismatch }\end{array}$ \\
\hline$\left[{ }^{*}\right.$ ONSET/X] & $\begin{array}{l}\text { - Niuafo'ou } \\
\text { - Pirahã }\end{array}$ & $\begin{array}{l}\text { (vacuously } \\
\text { satisfied) }\end{array}$ & $\begin{array}{l}\text { (hard to } \\
\text { distinguish } \\
\text { from other } \\
\text { constraints) }\end{array}$ \\
\hline HaVECPLACE & $\begin{array}{l}\text { domain } \\
\text { mismatch }\end{array}$ & $\begin{array}{l}\text { domain } \\
\text { mismatch }\end{array}$ & - Chamicuro \\
\hline HAVESTRESS & $\begin{array}{l}\text { (vacuously } \\
\text { satisfied) }\end{array}$ & $\begin{array}{l}\text { domain } \\
\text { mismatch }\end{array}$ & $\begin{array}{l}\text { domain } \\
\text { mismatch }\end{array}$ \\
\hline
\end{tabular}

In the matter of domain mismatches versus well-formed $\mathbf{M} / \mathbf{s t r}$ constraints, the *PEAK/X subhierarchy is an interesting case. The focus of *PEAK/X (shown in bold in (71)) includes reference to both a segment and a syllable, because a syllable peak is crucially identified as a segment that has a particular relationship to a syllable. 
(71) $*$ PEAK/X For every segment $a$ that is the head of some syllable $x$, $|a|>\mathrm{X}$

where $|y|$ is the sonority of segment $y$

$\mathrm{X}$ is a particular step on the segmental sonority scale

Thus, relativization to both a syllable-sized strong position (72a) and a segmentsized strong position (72b) is possible.

(72) Positional versions of *PEAK/X

(a) $\left[{ }^{*} \mathrm{PEAK} / \mathrm{X}\right] /$ o $\quad$ For every segment $a$ that is the head of some syllable $x$, if $x$ is a ó, then $|a|>\mathrm{X}$

where $|y|$ is the sonority of segment $y$

$\mathrm{X}$ is a particular step on the segmental sonority scale

(b) $\left[{ }^{*} \mathrm{PEAK} / \mathrm{X}\right] / \mathrm{V}$ : For every segment $a$ that is the head of some syllable $x$, if $a$ is a V:, then $|a|>\mathrm{X}$

where $|y|$ is the sonority of segment $y$

$\mathrm{X}$ is a particular step on the segmental sonority scale

As seen from the discussion of Zabiče Slovene, Mokshan Mordwin, and rhotic dialects of English, in which [*PEAK/X]/ó is active (\$3.2.1.3), and Yawelmani, in which $\left[{ }^{*} \mathrm{PEAK} / \mathrm{X}\right] / \mathrm{V}$ : is active (\$3.3), these two relativized versions of the ${ }^{*} \mathrm{PEAK} / \mathrm{X}$ subhierarchy are both attested.

In conclusion, the languages discussed in this chapter support the model of positional augmentation constraints developed in Chapter 2, according to which the Prominence Condition is the only constraint filter that is relevant for $\mathbf{M} / \mathbf{s t r}$ constraints on the phonetically strong positions stressed syllable, long vowel, and onset/released consonant. That is, any markedness constraint can be relativized to a phonetically strong position, as long as the basic criterion for possible $\mathbf{M} / \mathbf{s t r}$ constraints-the Prominence Condition-is met. A wide variety of augmentation effects are therefore observed in these positions. Of course, the kinds of augmentation phenomena that affect long vowels and onsets are necessarily limited to phenomena that can affect vowels and consonants respectively; other positional augmentation constraints for these positions lead to a domain mismatch. However, 
the comparatively unrestricted nature of $\mathbf{M} / \mathbf{s t r}$ constraints for phonetically strong positions (assuming only satisfaction of the Prominence Condition) is seen in the great variety of augmentation phenomena affecting stressed syllables. The syllabic size of this position makes it compatible with augmentation effects involving consonantal, vocalic, and prosodic features.

The varied combination of phonetically strong positions with augmentation constraints stands in contrast to the restricted augmentation possibilities for psycholinguistically strong positions, examined in the following chapter. The set of legitimate $\mathbf{M} / \mathbf{s t r}$ constraints for psycholinguistically strong positions is restricted not only by the Prominence Condition, but also by the Segmental Contrast Condition (\$2.4.1), which basically prohibits augmentation constraints from being relativized to psycholinguistically strong positions if such constraints would result in the neutralization of lexical contrasts in those positions. As a result, even though the psycholinguistically strong positions-initial syllable and root-are domains large enough for all the augmentation constraints enumerated in (70) to apply, the actual number of attested $\mathbf{M} / \mathbf{s t r}$ constraints for these two positions is somewhat smaller. 


\section{Augmentation of Psycholinguistically Strong Positions}

\subsection{Introduction}

The strong positions discussed in Chapter 3 have a special phonological status because of their phonetic salience. As demonstrated most thoroughly by Beckman (1998; see also Beckman 1997; Casali 1996, 1997), there are also positions that have the status of phonologically strong positions, and the concomitant ability to resist positional neutralization, because of their psycholinguistic salience-namely, the root and the morphological word (MWd)-initial syllable. ${ }^{1}$

The theory developed in Chapter 2 predicts that these psycholinguistically strong positions (abbreviated $\Psi_{s t r}$ ), being strong positions, should be eligible for positional augmentation constraints, and $\mathbf{M} / \mathbf{s t r}$ constraints for these positions are in fact attested (examples are discussed in $\$ 4.2$ ). The theory also predicts that, because only psycholinguistically strong positions are subject to the Segmental Contrast Condition ( $\$ 2.4)$, there should be fewer $\mathbf{M} / \Psi$ str than $\mathbf{M} / \Phi$ str constraints attested (where $\Phi_{s t r}$ are phonetically strong positions such as stressed syllable, long vowel, and onset/released consonant). Specifically, psycholinguistically strong positions are particularly important in the initial stages of word recognition, so there is substantive pressure against the neutralization of contrasts in those positions-even neutralization of the prominence-enhancing kind-because it

1. Beckman $(1997,1998)$ proposes that the relevant "initial" syllable for positional faithfulness constraints is the root-initial syllable. See $\S 4.4$ for discussion. 
would be inefficient for word recognition if too many contrasts were neutralized in these important positions. This substantive pressure is formalized as the Segmental Contrast Condition, a filter that rules out $\mathbf{M} / \Psi$ str constraints if their satisfaction would have the effect of neutralizing contrasts of the kind that are relevant for earlystage word recognition.

Indeed, empirically, the psycholinguistically strong positions do not exhibit the same wide range of augmentation effects that the phonetically strong positions show. For example, although there is a $\left[{ }^{*} \mathrm{PEAK} / \mathrm{X}\right] / \sigma$ constraint subhierarchy (§3.2.1.3), there is no evidence of an analogous $\left[{ }^{*} \operatorname{PEAK} / \mathrm{X}\right] / \sigma_{1}$ subhierarchy. The *PEAK/X constraint subhierarchy qualifies as prominence-enhancing (\$2.3.2.2), and it can obviously be relativized to a syllable-sized domain given $\left[{ }^{*} \mathrm{PEAK} / \mathrm{X}\right] / \sigma$, so the existence of a $\left[{ }^{*} \mathrm{PEAK} / \mathrm{X}\right] / \sigma_{1}$ subhierarchy would be compatible with $\mathbf{M} / \mathbf{s t r}$ constraint construction and the Prominence Condition. Nevertheless, $\left[{ }^{*} \mathrm{PEAK} / \mathrm{X}\right] / \sigma_{1}$ is not attested. Other examples of $\mathbf{M} / \Psi$ str constraints that satisfy the Prominence Condition but are not empirically attested include HEAVYo/ $\sigma_{1}$ and HAVECPLACE/Root; see chart (47) in $\$ 2.3 .3$ for a general comparison of attested $\mathbf{M} / \Psi$ str and $\mathbf{M} / \Phi$ str constraints.

The Segmental Contrast Condition correctly predicts that empirically attested

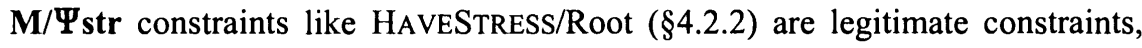
because stress (even when lexically contrastive) does not play the same role in early-stage word recognition that segmental contrasts play, and so augmenting even a psycholinguistically strong position with this property does not hamper the recognition of words. But something like the ${ }^{*} \mathrm{PEAK} / \mathrm{X}$ subhierarchy is relevant for segmental features rather than prosodic properties. Requiring initial syllables to have highly sonorant nuclei (as by a positional $\left[{ }^{*} \mathrm{PEAK} / \mathrm{X}\right] / \sigma_{1}$ subhierarchy) would reduce the number of segmental contrasts that are available in initial syllables, so this is the kind of constraint that the Segmental Contrast Condition rejects.

Finally, there is one case in which an $\mathbf{M} / \Psi$ str constraint is able to pass the Segmental Contrast Condition despite reference to a contrast that is relevant for early-stage word recognition: when satisfaction of the constraint would affect the left edge of the initial syllable. This aspect of the Segmental Contrast Condition also reflects substantive considerations, because the satisfaction of such constraints aids in demarcating the beginnings of words, and the segmentation (i.e., separation) of words in the speech stream is an important and difficult part of speech perception

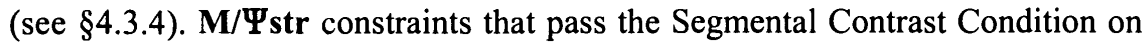
these grounds include ONSET $/ \sigma_{1}(\S 4.2 .1 .1)$ and the $\left[{ }^{*} \mathrm{ONSET} / \mathrm{X}\right] / \sigma_{1}$ subhierarchy (\$4.2.1.2).

The proposal summarized above has been presented in Chapter 2. The goal of the current chapter is to support this proposal, and explore some of its implications, by discussing in more depth various matters relating to the Segmental Contrast 
Condition and the augmentation of psycholinguistically strong positions. $\S 4.2$ presents case studies of languages in which $\mathbf{M} / \Psi$ str constraints are active; some of these case studies also have implications for subsyllabic structure and for details of the formulation of ${ }^{*}$ ONSET/X constraints. $\$ 4.3$ reviews evidence from psycholinguistic studies in support of the claims made in Chapter 2 about the substantive basis for the Segmental Contrast Condition: that the positions initial syllable and root have a particularly important status in lexical organization and early-stage word recognition; that the stressed syllable does not have the same status (although it is important for other aspects of speech perception); and that information about prosodic properties such as stress and perhaps also tone is not used to constrain early-stage word recognition in the same way that segmental features are used. $\$ 4.4$ examines the claim that "initial syllable" is best understood as "morphological-word-initial syllable," considering alternatives (particularly "root-initial syllable" (Beckman 1998) and "prosodic-word-initial syllable") and showing that the choice of MWd-initial syllable is the most consistent, theoretically and empirically, with the general pattern of attested initial-syllable augmentation effects. Conclusions are presented in $\S 4.5$.

\subsection{Exemplification of $M / \Psi$ str constraints}

This section demonstrates that augmentation constraints relativized to psycholinguistically strong positions play a crucial role in phonological patterning. Languages exemplifying initial-syllable augmentation constraints and root augmentation constraints are discussed in $\S 4.2 .1$ and $\S 4.2 .2$ respectively. $\S 4.2 .3$ then examines the typology of attested $M / \Psi$ str constraints and shows how the Segmental Contrast Condition (discussed more thoroughly in \$4.3) restricts augmentation constraints for psycholinguistically strong positions.

\subsubsection{Positional augmentation in initial syllables}

The initial syllable $\left(\sigma_{1}\right)$ is identified by Beckman $(1995,1997,1998)$ as a strong position. Phonologically, there is good evidence that the initial syllable has special status, since there are many languages in which it resists processes of positional neutralization (see, e.g., Trubetzkoy 1939; Steriade 1993; Beckman 1995, 1997, 1998; Casali 1996, 1997). The external justification that Beckman $(1997,1998)$ gives for the special status of the initial syllable is its importance in speech 
perception and language processing. (The discussion in $\S 4.3$ below argues that it is specifically an importance in early-stage word recognition that is crucial).

Since it is a strong position, the initial syllable is predicted to undergo positional augmentation in addition to resisting positional neutralization. This section presents examples of augmentation constraints that are relativized to this position: ONSET $/ \sigma_{1}(\$ 4.2 .1 .1)$ and the $\left[{ }^{*} \mathrm{ONSET} / \mathrm{X}\right] / \sigma_{1}$ constraint subhierarchy (§4.2.1.2, where some theoretical implications for syllable structure are also discussed).

Beckman $(1997,1998)$ proposes that what "initial syllable" means is rootinitial syllable, a choice that is consistent with the phonological patterns that she examines. The initial-syllable augmentation effects discussed here, on the other hand, are most compatible with the designation of "morphological word (MWd)initial syllable" as the relevant strong position. Explicit discussion of this choice, and of the broader implications of various possible definitions of "initial syllable," is postponed until $\S 4.4$ below, since the purpose of the current section is to present natural-language examples of augmentation phenomena in initial syllables.

\subsubsection{Obligatory onsets in initial syllables: ONSET/ $\sigma_{I}$}

In some languages, the requirement that syllables must have onsets holds more strictly of initial syllables than of other syllables; onsetless syllables are tolerated medially, but not initially. The special demand for initial onsets is accounted for with an initial-syllable-specific version of the general augmentation constraint ONSET (the particular formulation of ONSET given here was introduced in \$2.3.2.3.2; the positional restrictor if-then clauses that correspond to each strong position were introduced in §2.3.3).

ONSET $/ \sigma_{1}$

For all syllables $x$, if $x$ is the leftmost syllable whose head is affiliated with MWd $m$, then $a \neq b$

where $a$ is the leftmost segment dominated by syllable $x$ $b$ is the head of syllable $x$

Since the constraint ONSET $/ \sigma_{1}$ is relativized to the strong position initial syllable, no violations are incurred by a medial syllable that lacks an onset. Languages that tolerate medial hiatus but not initial vowels have the ranking in (2).

$$
\text { ONSET } / \sigma_{1} \gg \text { FAITH } \gg \text { ONSET }
$$


General ONSET is ranked low, which allows vowel-initial syllables generally, but specific ONSET $/ \sigma_{1}$ is ranked high, ensuring that all initial syllables do have onsets.

This section discusses two languages that have a specific onset requirement for initial syllables, Arapaho (Salzmann 1956) and Guhang Ifugao (Newell 1956, Landman 1999). Other languages that require onsets in initial syllables, but not in all syllables, include Hausa (Greenberg 1941), Guarani (Gregores \& Suarez 1967), and Tabukang Sangir (Maryott 1961).

According to Salzmann (1956), the Northern Arapaho dialect of Arapaho (Algonquian) is an example of a language that tolerates onsetless syllables medially, but not word-initially. Examples of medial syllables without onsets are given in (3).

$$
\begin{aligned}
& \text { Medial onsetless syllables (Salzmann 1956:53-4) }{ }^{2} \\
& \text { čé.či.no.hú:.으 } \\
& \text { noh.?ó.ex.sei.hí.hi? 'firefly' } \\
& \text { wo.ró.u‥so: 'kitten' } \\
& \text { 'chicken hawk (obviative)' }
\end{aligned}
$$

However, in initial position, onsetless syllables are not tolerated: vowels "are found only medially and finally, thus contrasting with consonants which are found also initially" (Salzmann 1956:51). All words start with one of the consonantal phonemes $/ \mathrm{b}, \mathrm{t}, \mathrm{k}, \mathrm{c}, \theta, \mathrm{s}, \mathrm{x}, \mathrm{h}, \mathrm{n}, \mathrm{w}, \mathrm{y} / \mathrm{.}^{3}$

2. Medial hiatus is not tolerated when the adjacent vowels are identical (this is presumably an OCP effect). For example, Salzmann (1956:53) states that in a sequence of like vowels VV, a tonal pattern in which the vowels are heterosyllabic, "the two vowels are peaks of two separate syllables marked with a y-like or h-like transition in the case of $i$, and an h-like transition in the case of the other three vowels [[e,u,o]-JLS]."

3. $\Pi /$ is also a phoneme of the language, but Salzmann (1956:50) notes that word-initial $/ ? /$ occurs in his corpus only in two interjections. 
(4) No vowel-initial words (Salzmann 1956:51, 53)

\begin{tabular}{|c|c|}
\hline bétee & '(human) heart' \\
\hline kétee & 'is it your heart?' \\
\hline čis & 'nighthawk' \\
\hline seeníwuú & 'lizards' \\
\hline xooó & 'skunk' \\
\hline hé $\theta$ & 'dog' \\
\hline nówo? & 'fish' \\
\hline wóto? & '(human) nape' \\
\hline
\end{tabular}

Because word-internal syllables can be vowel-initial (3), the constraint ONSET is dominated by faithfulness constraints such as MAX-SEG (which penalizes deletion) and DEP-SEG (which penalizes epenthesis) (5).

(5) Segmental correspondence (faithfulness) constraints (after McCarthy \& Prince 1995)

MAX-SEG Every input segment has an output correspondent

DEP-SEG Every output segment has an input correspondent

The interaction among ONSET, MAX-SEG, and DEP-SEG is shown in (6).

(6) Medial onsetless syllables are tolerated

\begin{tabular}{|c|c|c|c|}
\hline /Čéčinohú:o/ & MAX-SEG & DEP-SEG & ONSET \\
\hline a. čé.či.no.hú:.o & & & \\
\hline 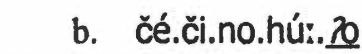 & & $* !$ & \\
\hline c. čé.či.no.hú:_ & $* !$ & & \\
\hline
\end{tabular}

However, as seen in (4) above, a stricter requirement holds of initial syllables: they must have onsets. No vowel-initial forms are allowed. Languages like Arapaho (and Guhang Ifugao; see below) thus present an interesting contrast to the more 
common pattern, found for example in Axininca Campa (Payne 1981; Spring 1990; McCarthy \& Prince 1993ab), in which onsetless syllables are tolerated only in initial position. In languages like Axininca, alignment constraints dominate ONSET (McCarthy \& Prince 1993ab), so that disrupting the alignment of the input material with the left edge of the prosodic word by means of epenthesis is worse than tolerating an onsetless syllable. This can be seen in (7), where failure to epenthesize an onset medially is fatal (7a), but epenthesizing an initial onset is also fatal (7b).

$$
\text { Initial onset epenthesis blocked in Axininca Campa }
$$
(adapted from McCarthy \& Prince 1993a:119)

/i-N-koma-i/ 'he will paddle' (epenthetic segments are in boldface)
\begin{tabular}{|r||c|c|c|}
\hline /i-N-koma-i/ & $\begin{array}{c}\text { ALIGN-L } \\
\text { (Stem, PrWd) }\end{array}$ & ONSET & DEP-SEG \\
\hline \hline a. in.ko.ma.i & & $* * !$ & \\
\hline b. tin.ko.ma.ti & $* !$ & & $* *$ \\
\hline c. in.ko.ma.ti & & $*$ & $*$ \\
\hline
\end{tabular}

By contrast, Arapaho-type languages seem to go out of their way to disrupt edge-alignment. Because these languages require onsets in initial position, an alignment-based analysis will not work. What can account for the Arapaho pattern is a constraint that specifically mandates the presence of an onset in the initial syllable: ONSET $/ \sigma_{1}(8)$. If this constraint dominates at least one faithfulness constraint, then initial syllables will always surface with an onset (9).

(8) ONSET/ $\sigma_{1} \quad$ For all syllables $x$, if $x$ is the leftmost syllable whose head is affiliated with MWd $m$, then $a \neq b$

where $a$ is the leftmost segment dominated by syllable $x$ $b$ is the head of syllable $x$ 
(9) Initial syllables always have onsets (hypothetical input)

\begin{tabular}{|c|c|c|c|c|}
\hline /oto?/ & ONSET/ $\sigma_{1}$ & MAX-SEG & DEP-SEG & ONSET \\
\hline a. oto? & $* !$ & & & $*$ \\
\hline (ख) b. hoto? & & & $*$ & \\
\hline (1) c. c. to? & & * & & 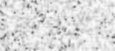 \\
\hline
\end{tabular}

Whether $(9 b)$ or $(9 c)$ - or indeed some other candidate not shown here-is the actual output that the grammar of Arapaho would produce for the hypothetical input /oto?/ will depend on the ranking relationships among MAX-SEG, DEP-SEG, and other faithfulness constraints, which cannot be precisely determined here. What matters for this discussion is that the faithful, onsetless candidate is less harmonic than other candidates, so an output with an onsetless initial syllable will never surface.

Guhang Ifugao (Newell 1956; Landman 1999) is another language that shows the effects of the ONSET $/ \sigma_{1}$ constraint. ${ }^{4}$ Onsetless syllables do occur in the language, as seen in (10). (The syllable breaks for the first five examples are given explicitly by Newell (1956:535); other examples are syllabified in accordance with his description of syllable structure. Note that either vowel in a VV sequence may bear stress, a fact which supports separate syllabification.)

4. Landman (1999) takes a different approach to Guhang Ifugao, proposing a constraint, MORPHEME-CONTIGUITY, that penalizes contiguity violations (including epenthesis, deletion, and metathesis) inside morphemes but is not violated by epenthesis at morpheme edges. 
(10) Medial onsetless syllables (Newell 1956:535, 538)

$\begin{array}{ll}\text { Ti.áb.ba? } & \text { 'I will carry (on back)' } \\ \text { bú.un } & \text { 'an Ifugao necklace' } \\ \text { ma.ná.an } & \text { 'remove' } \\ \text { ma.ga.á.tan } & \text { 'clean (by cutting)' } \\ \text { ba.bá.ị } & \text { 'female' } \\ \text { ha.í.tan } & \text { 'whetstone' } \\ \text { hé.ẹp } & \text { 'sunflower' } \\ \text { bu.ma.nú.ot } & \text { 'smolder' } \\ \text { ma.ni.go.á? } & \text { 'I'm looking for' }\end{array}$

However, vowel-initial syllables are not permitted in word-initial position (11).

(11) Initial onsets are obligatory (Newell 1956:534-6)

$\begin{array}{ll}\text { (a) tin.ní.go } & \text { 'saw' } \\ \text { gád.den } & \text { 'goat' } \\ \text { mun.bán.nad } & \text { 'return' } \\ \text { ní.lig } & \text { 'yard' } \\ \text { lu.má.hu } & \text { 'hot' } \\ \text { hi.dí } & \text { 'there (far)' } \\ \text { ya.gu.yá } & \text { 'why' } \\ \text { wá.da } & \text { 'there is' } \\ \text { ?u.húp } & \text { 'late, long time' } \\ \text { 7ín.ni } & \text { 'baby girl' } \\ \text { ?ad.dá.ya } & \text { 'sky' } \\ & \\ \text { (b) *uhup } & \\ \text { *inni } & \\ \text { *addaya } & \end{array}$

Just as for Arapaho, an analysis that accounts for the facts of Guhang Ifugao is one in which ONSET $/ \sigma_{1}$ outranks (at least one of) the faithfulness constraints MAX-SEG and DEP-SEG (12). 
(12) Initial syllables always have onsets (hypothetical input)

\begin{tabular}{|c|c|c|c|c|}
\hline /uhup/ & ONSET/ $\sigma_{1}$ & MAX-SEG & DEP-SEG & ONSET \\
\hline a. u.hup & $* !$ & & & * \\
\hline (\$) b. ?u.hup & & & * & \\
\hline (®) c. _hup & & $*$ & & \\
\hline
\end{tabular}

Again, as for Arapaho, the evidence introduced here does not determine whether onsetless initial syllables are repaired by means of (consonant) epenthesis or (vowel) deletion; the choice between (12b) and (12c) will depend on the relative ranking of the faithfulness constraints. (Landman (1999) suggests that there is evidence, from orthographic practice and comparison with related languages, that glottal-stop epenthesis is the chosen "repair" for a potential onsetless initial syllable, so (12b) may be the actual output.)

The languages examined in this section, Arapaho and Guhang Ifugao, are languages with mandatory onsets only in initial syllables. Such languages provide evidence for the positional augmentation constraint ONSET $/ \sigma_{1}$. In such a language, ONSET $/ \sigma_{1}$ outranks at least one of the faithfulness constraints that would otherwise protect initial onsetless syllables, such as DEP-SEG or MAX-SEG. However, the general ONSET constraint is dominated by all relevant faithfulness constraints; as a result, non-initial syllables may remain onsetless.

The following section examines phenomena that motivate another family of positional augmentation constraints for the strong position $\sigma_{1}$ : the $\left[{ }^{*} \mathrm{ONSET} / \mathrm{X}\right] / \sigma_{1}$ subhierarchy.

\subsubsection{High-sonority onsets banned in initial syllables: $\left[{ }^{*}\right.$ ONSET/X]/$\sigma_{I}$}

In certain languages, initial-syllable onsets that are high in sonority, such as glides or liquids, are not allowed. ${ }^{5}$ To account for languages with special onset-sonority restrictions for initial syllables, it is necessary to recognize a specific version of the *ONSET/X constraint subhierarchy relativized to initial syllables, $\left[{ }^{*} \mathrm{ONSET} / \mathrm{X}\right] / \sigma_{1}$.

5. In such languages, initial onsetless syllables may be tolerated. This is to be expected, since ONSET and * ONSET/X are formally independent and the ranking between them is not universally fixed (\$2.3.2.3.3). 
This section examines three different sonority-sensitive restrictions on initialsyllable onsets: a ban on rhotic and glide onsets in initial syllables, a ban on all liquid onsets in initial syllables, and a ban on specifically rhotic onsets in initial syllables. These three types are exemplified by Campidanian Sardinian ( $\$ 4.2 .1 .2 .1)$, by Mongolian, Kuman, Guugu Yimidhirr, and Pitta-Pitta (\$4.2.1.2.2), and by Mbabaram (\$4.2.1.2.3) respectively.

The general *ONSET/X subhierarchy, a universally ranked set of constraints banning onset consonants of particular levels of sonority, was discussed in $\S 2.3 .2 .3 .3$. The initial-syllable-specific version of this constraint subhierarchy is given in (13).

$\left[{ }^{*} \mathrm{ONSET} / \mathrm{X}\right] / \sigma_{1}$ For every segment $a$ that is the leftmost pre-moraic segment of some syllable $x$, if $x$ is the leftmost syllable whose head is affiliated with MWd $m$, then $|a|<\mathrm{X}$

where $|y|$ is the sonority of segment $y$

$\mathrm{X}$ is a particular step on the segmental sonority scale

The particular values assigned to $X$ in (13), and therefore the specific formulations of the individual constraints that make up the subhierarchy, depend on the composition of the segmental sonority hierarchy. The sonority hierarchy assumed here is that shown in (14) (see $\S 2.3 .2 .2$ for discussion). Some of the languages discussed in this section, Campidanian Sardinian (\$4.2.1.2.1) and Mbabaram ( $\$ 4.2 .1 .2 .3$ ), further support the division of the class of liquids into rhotics and laterals, because they prohibit rhotics but allow laterals as the onsets of initial syllables.

$$
\begin{aligned}
& \text { Segmental sonority scale (consonantal portion) } \\
& \text { glides }>\text { rhotics }>\text { laterals }>\text { nasals }>\text { voiced obstruents }>\text { voiceless } \\
& \text { obstruents }
\end{aligned}
$$

When this sonority scale is applied to the constraint formulation in (13) above, the following universal subhierarchy is derived (where $D$ and $T$ are abbreviations for voiced and voiceless obstruents respectively).

$$
\begin{aligned}
& {\left[{ }^{*} \mathrm{ONS} / \mathrm{GLI}\right] / \sigma_{1} \gg\left[{ }^{*} \mathrm{ONS} / \mathrm{RHO}\right] / \sigma_{1}>\left[{ }^{*} \mathrm{ONS} / \mathrm{LAT}\right] / \sigma_{1}>>\left[{ }^{*} \mathrm{ONS} / \mathrm{NAS}\right] / \sigma_{1}} \\
& >>\left[{ }^{*} \mathrm{ONS} / \mathrm{D}\right] / \sigma_{1} \gg>\left[{ }^{*} \mathrm{ONS} / \mathrm{T}\right] / \sigma_{1}
\end{aligned}
$$


The analyses proposed in this section also have implications for the formulation of general and positional *ONSET/X constraints. Languages in which syllable-initial glides are syllabified as true onset consonants must ban initial glides when they ban initial liquids, because of the universal ranking [ $\left.{ }^{*} \mathrm{ONS} / \mathrm{GLI}\right] / \sigma_{1} \gg\left[{ }^{*} \mathrm{ONS} / \mathrm{RHO}\right] / \sigma_{1}$ $>\left[{ }^{*} \mathrm{ONS} / \mathrm{LAT}\right] / \sigma_{1}$. An example of a language that follows this pattern is the Sestu dialect of Campidanian Sardinian (\$4.2.1.2.1). Thus, languages that ban word-initial rhotics (or liquids generally) while tolerating word-initial glides, such as Mongolian, Kuman, Guugu Yimidhirr, Pitta-Pitta, and Mbabaram, provide evidence that not all syllable-initial glides are structurally onsets. This point will be taken up at the end of the onset-sonority discussion, in $\$ 4.2 .1 .2 .4$.

\subsection{Initial glide and rhotic onsets banned in Campidanian Sardinian}

In the Sestu dialect of Campidanian Sardinian (Bolognesi 1998), ${ }^{6}$ there is a prohibition against initial onsets that consist solely of a glide or of the liquid [r]. Sestu Campidanian thus provides an example of a language that bans both liquid (rhotic, in this case) and glide onsets together. This subsection first gives an overview of the relevant data and then presents an analysis that makes crucial use of the positional augmentation constraints $\left[{ }^{*} \mathrm{ONSET} / \mathrm{GLI}\right] / \sigma_{1}$ and [ $\left.{ }^{*} \mathrm{ONSET} / \mathrm{RHO}\right] / \sigma_{1}$.

Where initial [r] would have been expected in Sestu and other Campidanian dialects, given the diachronic or loanword source of a particular word, the liquid is instead preceded by a low vowel (and is usually geminate). ${ }^{7}$

(16) Potential [r]-initial words have prothetic vowels (Bolognesi 1998:42)

(a) Regular outcomes of diachronic change from Latin/Romance

$\begin{array}{lll}\text { ar:oza } & \text { 'rose' } & <\text { Latin rosa } \\ \text { ariu } & \text { 'river/creek' } & <\text { Latin rivus } \\ \text { ariana } & \text { 'frog' } & \text { < Latin } \text { rana } \\ \text { ar:ußiu 'red' } & \text { < Latin rubeum } \\ \text { ar:oða } & \text { 'wheel' } & \text { < Latin rota }\end{array}$

6. Thanks to Paul de Lacy for bringing this example and reference to my attention.

7. According to Bolognesi (1998), the initial [a] is optional for some recent loanwords from Italian. 
(b) Loanwords from Italian

$$
\begin{array}{ll}
\text { ariik:u 'rich' } & <\text { Italian ricco } \\
\text { ar:aðiu 'radio' } & \text { < Italian radio }
\end{array}
$$

However, syllable-initial [r] is possible in syllables that are not word-initial $(17 \mathrm{a}){ }^{8}$ and [r] can appear as part of a complex onset (17b), even in word-initial syllables.

$$
\text { Licit occurrences of onset [r] }
$$

(a) Onset [r] in non-initial syllables (Bolognesi 1998:37, 39, 496, 498)

$\begin{array}{ll}\text { dzeneru } & \text { 'son-in-law' } \\ \text { dzị̌u } & \text { 'jar' } \\ \text { ankora } & \text { 'still' } \\ \text { pu[̦u } & \text { 'also' } \\ \text { naraða } & \text { 'call-3sG-PST' }\end{array}$

(b) [r] in complex onsets (Bolognesi 1998: 31, 44, 45, 496, 497, 502)

$\begin{array}{ll}\text { tronu } & \text { 'thunder' } \\ \text { trinku } & \text { 'scar' } \\ \text { prenu } & \text { 'full' } \\ \text { grandu } & \text { 'great' } \\ \text { frisku } & \text { 'fresh air' } \\ \text { at:rֵu } & \text { 'other' } \\ \text { sempri } & \text { 'always' }\end{array}$

Sestu Campidanian also disallows glide-initial words, again with the exception of certain very recent loanwords like yoghurt [jo yurtu] and whisky (no transcription given). According to Bolognesi (1998:44), word-initial [j] is relatively uncommon in the Campidanian dialects in general, occurring in about four forms. But Sestu disallows initial [j] even in these forms. There is a prothetic [a] in two of the four

8. Bolognesi (1998:27) explicitly states that $/ \mathrm{r} /$ does not show any allophonic alternations. However, he sometimes-although not consistently-gives intervocalic $/ r /$ as a surface tap [r] in his phonetic transcriptions. Forms shown here follow Bolognesi's transcriptions. 
cases where other Campidanian dialects have initial [j] (34a). (In the other two cases, the initial consonant is an affricate in Sestu (34b) or the Sestu form is not given (34c).)

Forms with initial [j] (Bolognesi 1998:44)

\section{Sestu form Other Campidanian dialects Gloss}
(a) ajaju
jaju
'grandfather'
ajaja
jaja
'grandmother'
(b) $d 3 u$
juu
'yoke'
(c) $<$ not given $>^{9}$
jos:u
'down'

As for initial [w], Bolognesi (1998:44) states that it occurs in word-initial position only in the single loanword whisky.

In addition to the forms in (18a), word-medial glide onsets appear in a few other forms.

Word-medial glide onsets (Bolognesi 1998:44-5)

$\begin{array}{ll}\text { maju } & \text { 'May' } \\ \text { eja } & \text { 'yes' } \\ \text { koja } & \text { 'wedding' } \\ \text { fawajt]u } & \text { 'liar' }\end{array}$

Thus, like [r], glides can be syllable onsets as long as they are not in initial position. But glides differ from [r] in one respect: in Sestu, glides do not appear with another onset consonant, so that there are.$C r V$. sequences but not $. C w / j V$. sequences. (This point becomes important in the discussion of syllabic positions for glides in $\$ 4.2 .1 .2 .4$ below.)

Rising diphthongs are allowed by syllable structure in some dialects of Campidanian, but they are normally prohibited in Sestu and other central dialects.

9. The Sestu form corresponding to [jos:u] 'down' is not given in Bolognesi's (1998:44-5) explicit discussion of initial glides, and it does not appear in either of the two long Sestu texts transcribed in Bolognesi's appendix. 
As a consequence the "Standard" Campidanian word 'kwaq.u ('horse') is realized as $k u^{\prime} a d \cdot u$ in the Sestu dialect: / $u$ / is short and unstressed, but distinctly longer than the corresponding glide. (Bolognesi 1998:24) ${ }^{10}$

In contrast to $[\mathrm{r}]$ and the glides, which do not appear in initial position, [1] can appear as a word-initial onset in Sestu Campidanian.

(20) Onset [1] in word-initial syllables (Bolognesi 1998:43-4)

$\begin{array}{ll}\text { luzi } & \text { 'light' } \\ \text { ledzu } & \text { 'ugly' } \\ \text { lat:i } & \text { 'milk' } \\ \text { luðu } & \text { 'mud' }\end{array}$

To summarize the patterns seen in the data introduced above: Sestu Campidanian does not tolerate glides or $[r]$ as independent onsets in initial syllables. However, these consonants can be onsets of medial syllables, which indicates that the sonority-based restrictions in question hold specifically of initial syllables. Finally, while [r] can form part of an onset cluster even in initial syllables, rising diphthongs (or complex onsets involving glides) are not permitted. The remainder of this subsection presents an analysis of initial-syllable onsets in Sestu, accounting for the special ban on high-sonority elements with constraints from the $\left[{ }^{*} \mathrm{ONSET} / \mathrm{X}\right] / \sigma_{1}$ constraint subhierarchy.

The consonants that may not be initial onsets in Sestu Campidanian are the glides, $[\mathrm{j}, \mathrm{w}]$, and the rhotic [r]. This pattern can be accounted for if the constraints $\left[{ }^{*} \mathrm{ONSET} / \mathrm{GLI}\right] / \sigma_{1}$ and $\left[{ }^{*} \mathrm{ONSET} / \mathrm{RHO}\right] / \sigma_{1}$ are ranked above at least one of the faithfulness constraints.

10. Rising diphthongs do appear from time to time in Bolognesi's (1998) phonetic transcriptions of the running texts. For example, the form /famil:ia/ is given as [(v)amil:ia], as expected, on p. 30, but as [(f:)amil:ia], with an apparent rising diphthong, on p. 45 (in both examples, sandhi processes have affected the word-initial fricative). Since Bolognesi so explicitly states that Sestu does not have rising diphthongs, presumably the unexpected glides in the transcriptions are to be taken as some sort of rapid-speech contraction. 
(21)

$[*$ ONSET/GLI $] / \sigma_{1}$

For every segment $a$ that is the leftmost pre-moraic segment of some syllable $x$, if $x$ is the leftmost syllable whose head is affiliated with $\mathrm{MWd} m$, then $|a|<\mathrm{GLI}$

where $|y|$ is the sonority of segment $y$

$\left[{ }^{*} \mathrm{ONSET} / \mathrm{RHO}\right] / \sigma_{1} \quad$ For every segment $a$ that is the leftmost pre-moraic segment of some syllable $x$, if $x$ is the leftmost syllable whose head is affiliated with MWd $m$, then $|a|<\mathrm{RHO}$

where $|y|$ is the sonority of segment $y$

Because Sestu words that correspond to glide- or [r]-initial words in other dialects or languages generally have initial [a] (and even very recent loanwords from Italian that begin with [r] have optional variants with an epenthetic [a]; Bolognesi 1998:43), it appears that the faithfulness constraint crucially dominated by these initial-syllable markedness constraints is DEP-SEG, which penalizes epenthesis.

The constraints $\left[{ }^{*} \mathrm{ONSET} / \mathrm{GLI}\right] / \sigma_{1}$ and $\left[{ }^{*} \mathrm{ONSET} / \mathrm{RHO}\right] / \sigma_{1}$ must also dominate ONSET $/ \sigma_{1}$ (as well as general ONSET), or the onsetless syllable created by [a]epenthesis would not be tolerated. DEP-SEG must dominate ONSET $/ \sigma_{1}$ as well, or an epenthetic onset would appear with the epenthetic [a]. The interactions among these constraints are shown in (22).

(22) Initial glides and [r] avoided

(a) [ajaju] 'grandfather'

\begin{tabular}{|c|c|c|c|c|}
\hline /jaju/ & $\begin{array}{l}{\left[{ }^{*} \text { ONS/ }\right.} \\
\mathrm{GLI}] / \sigma_{1} \\
\end{array}$ & $\begin{array}{l}{\left[{ }^{*} \text { ONS/ }\right.} \\
\text { RHO }] / \sigma_{1} \\
\end{array}$ & $\begin{array}{l}\text { DEP- } \\
\text { SEG } \\
\end{array}$ & ONSET $/ \sigma_{1}$ \\
\hline a. jaju & $* !$ & & +4 & \\
\hline b. ajaju & & & $*$ & 23 \\
\hline c. tajaju & & & $* * !$ & \\
\hline
\end{tabular}


(b) [ar:っða] 'wheel' ${ }^{11}$

\begin{tabular}{|c|c|c|c|c|}
\hline /roда/ & $\begin{array}{l}{\left[{ }^{*} \mathrm{ONS} /\right.} \\
\mathrm{GLI}] / \sigma_{1}\end{array}$ & $\begin{array}{l}{\left[{ }^{*} \text { ONS/ }\right.} \\
\text { RHO }] / \sigma_{1} \\
\end{array}$ & $\begin{array}{l}\text { DEP- } \\
\text { SEG } \\
\end{array}$ & ONSET $/ \sigma_{1}$ \\
\hline a. roða & & *! & & \\
\hline b. ar:っða & & & $*$ & $*$ \\
\hline c. tar:oða & & & $* * !$ & \\
\hline
\end{tabular}

Although DEP-SEG is dominated by $\left[{ }^{*}\right.$ ONSET $\left./ \mathrm{GLI}\right] / \sigma_{1}$ and $\left[{ }^{*} \mathrm{ONSET} / \mathrm{RHO}\right] / \sigma_{1}$, it dominates the other constraints in the subhierarchy, including [ ${ }^{*}$ ONSET/LAT] $/ \sigma_{1}$.

$$
\begin{array}{ll}
{\left[{ }^{*} \text { ONSET } / \text { LAT }\right] / \sigma_{1}} & \text { For every segment } a \text { that is the leftmost pre-moraic } \\
\text { segment of some syllable } x, \text { if } x \text { is the leftmost } \\
\text { syllable whose head is affiliated with MWd } m \text {, then } \\
|a|<\text { LAT }
\end{array}
$$

where $|y|$ is the sonority of segment $y$

Therefore, initial liquid onsets (and less sonorous initial segments) appear faithfully in output forms.

11. While the constraints introduced thus far are sufficient to account for the glide-initial cases, something more will need to be said about the [r]-initial cases, given that a form like /roða/ surfaces as [ar:oða], with gemination of the liquid. Some constraint must be active that prefers a candidate with gemination over the more faithful candidate *[arəða]. Intuitively, one suspects that there may be an opaque interaction here-perhaps an underlyingly wordinitial [r] resists being realized as a flap even though it is intervocalic on the surface, and gemination is the strategy chosen to avoid flaps, if indeed flaps are mandatory in intervocalic environments; see footnote 8 . 
(24) Initial [1] surfaces: [luzi] 'light'

\begin{tabular}{|c||c|c|c|c|}
\hline /luzi/ & $\begin{array}{c}{\left[{ }^{*} \mathrm{ONS} /\right.} \\
\mathrm{GLI}] / \sigma_{1}\end{array}$ & $\begin{array}{c}{\left[{ }^{*} \mathrm{ONS} /\right.} \\
\text { RHO }] / \sigma_{1}\end{array}$ & DEP-SEG & $\begin{array}{c}{\left[{ }^{*} \mathrm{ONS} /\right.} \\
\mathrm{LIO}] / \sigma_{1}\end{array}$ \\
\hline \hline a. luzi & & & & $*$ \\
\hline b. aluzi & & & $* !$ & \\
\hline
\end{tabular}

Since all relevant faithfulness constraints dominate [ $\left.{ }^{*} \mathrm{ONSET} / \mathrm{LAT}\right] / \sigma_{1}$, but [*ONSET/RHO] $/ \sigma_{1}$ crucially dominates at least one of the faithfulness constraints, the two constraints [ $\left.{ }^{*} \mathrm{ONSET} / \mathrm{RHO}\right] / \sigma_{1}$ and $\left[{ }^{*} \mathrm{ONSET} / \mathrm{LAT}\right] / \sigma_{1}$ must be recognized as distinct members of the $\left[{ }^{*} \mathrm{ONSET} / \mathrm{X}\right] / \sigma_{1}$ hierarchy. A unitary [ $\left.{ }^{*} \mathrm{ONSET} / \mathrm{LIQ}\right] / \sigma_{1}$ constraint, that makes reference to rhotics and laterals together, cannot capture the pattern of permissible initial onsets seen in Sestu Campidanian. (The same point can be made about Mbabaram, discussed in $\$ 4.2 .1 .2 .3$ below.)

A final point to note is that, because the formulation of *ONSET/X constraints makes them relevant only for the leftmost (pre-moraic) segment of the syllable, [*ONSET $/ \mathrm{RHO}] / \sigma_{1}$ correctly fails to penalize initial $\mathrm{Cr}$ - clusters, which are attested in Sestu Campidanian. The leftmost element of a syllable such as [tro] in (25) is [t], not $[\mathrm{r}]$, so it does not violate $\left[{ }^{*} \mathrm{ONSET} / \mathrm{RHO}\right] / \sigma_{1}$ as the syllable [ro] would do.

Initial $\mathrm{Cr}$ - onsets are allowed: [tronu] 'thunder'

\begin{tabular}{|c||c|c|c|}
\hline /tronu/ & {$\left[{ }^{*} \mathrm{ONS} / \mathrm{GLI}\right] / \sigma_{1}$} & {$\left[{ }^{*} \mathrm{ONS} / \mathrm{RHO}\right] / \sigma_{1}$} & DEP-SEG \\
\hline \hline a. tronu & & & \\
\hline b. atronu & & & $* !$ \\
\hline c. taronu & & & $* !$ \\
\hline
\end{tabular}

As noted above, however, Sestu does not allow rising diphthongs; that is, syllables like *[tja] or *[twa] are prohibited. These syllables satisfy [*ONSET/GLI], just as the attested syllable [tro] satisfies [ ${ }^{*}$ ONSET/RHO], because the glide is not leftmost in the syllable. Therefore, another constraint must be responsible for ruling out such syllables. This point is considered again in $\S 4.2 .1 .2 .4$ below, where various matters pertaining to glides and onsets are addressed.

In conclusion, the initial-syllable-specific constraint subhierarchy [*ONSET $/ \mathrm{X}] / \sigma_{1}$ accounts for the prohibition of initial $[\mathrm{j}, \mathrm{w}, \mathrm{r}]$ in Sestu Campidanian. 
[ $\left.{ }^{*} \mathrm{ONSET} / \mathrm{GLI}\right] / \sigma_{1}$ and $\left[{ }^{*} \mathrm{ONSET} / \mathrm{RHO}\right] / \sigma_{1}$ are ranked above a faithfulness constraint, so glide and [r] onsets are avoided. However, [ ${ }^{*}$ ONSET $/$ LAT $] / \sigma_{1}$ and all lowerranked constraints in the $\left[{ }^{*} \mathrm{ONSET} / \mathrm{X}\right] / \sigma_{1}$ hierarchy are dominated by all relevant faithfulness constraints, so that other consonants, including [1], are permissible initial onsets.

\subsection{Initial liquid onsets banned}

This section discusses four languages with a different kind of initial-syllable onset sonority restriction. Mongolian, Kuman, Guugu Yimidhirr, and Pitta-Pitta, each discussed in Walsh Dickey 1997, ban all liquid onsets-including laterals as well as rhotics - in initial syllables. (Unlike Sestu Campidanian, these languages do allow word-initial glides. The implicational relationship between liquids and glides as admissible onsets is discussed in §4.2.1.2.4 below.)

First, the crucial initial-syllable restrictions in each language are presented. Then, an analysis is proposed that accounts for these languages with the positional augmentation constraints [ $\left.{ }^{*} \mathrm{ONSET} / \mathrm{RHO}\right] / \sigma_{1}$ and $\left[{ }^{*} \mathrm{ONSET} / \mathrm{LAT}\right] / \sigma_{1}$.

In (Khalkha) Mongolian, as in most Altaic languages, there is a ban on liquids in word-initial position (Poppe 1970, Ramsey 1987), ${ }^{12}$ although they may appear medially and finally (26). Ramsey (1987:205) describes [1] as a dental lateral, and [r] as a dental "flap or trill."

Medial and final liquids (Ramsey 1987:205-7)

$\begin{array}{llll}\text { dalae } & \text { 'sea' } & \text { zurag } & \text { 'picture' } \\ \text { üül } & \text { 'cloud' } & \text { xar } & \text { 'black' } \\ \text { emeel } & \text { 'saddle' } & \text { tenger } & \text { 'sky' }\end{array}$

Other consonants ${ }^{13}$ may appear initially (27). Vowel-initial words are also permitted (28).

12. According to Ramsey (1987:206), the ban on initial [r] is absolute, but initial [l] does occur in a small number of loanwords. This difference between [ $\mathrm{r}]$ and $[\mathrm{l}]$ may well reflect rhotics' higher sonority.

13. Ramsey (1987:205) states that [w] occurs only in loanwords, but he does not indicate whether or not $[w]$ is a possible initial onset. 
(27) Initial consonants (Ramsey 1987:205-9)

$\begin{array}{llll}\text { tenger } & \text { 'sky' } & \text { xüxe } & \text { 'blue' } \\ \text { baatar } & \text { 'hero' } & \text { zuraba } & \text { 'painted' } \\ \text { gar } & \text { 'hand' } & \text { maxa } & \text { 'meat, fish' } \\ \text { Jönö } & \text { 'night' } & \text { jaba } & \text { 'go!' }\end{array}$

(28) Vowel-initial words (Ramsey 1987:205-9)

$\begin{array}{ll}\text { ulaan } & \text { 'red' } \\ \text { emeel } & \text { 'saddle' } \\ \text { onoqu } & \text { 'to understand' } \\ \text { axa } & \text { 'elder brother' }\end{array}$

Thus, the class of liquids is systematically excluded from the word-initial onset position in Mongolian.

Another language in which initial liquids are banned is Kuman (Papuan; Trefry 1969, Lynch 1983, Blevins 1994). In this language, as analyzed by Lynch (1983 [cited in Blevins 1994]), there are two contrastive liquids, a velar lateral [L] (which can coalesce with a following [i] to form an alveolar lateral [1]), and an alveolar flap $[r]$ that is in complementary distribution with [t]. No liquid segment occurs wordinitially in Kuman; the [r] [t] phoneme surfaces as [t] whenever it is word-initial (although [r] appears prevocalically in all other cases), and [L] [l] does not appear in initial position at all. However, both liquids occur medially and finally (29).

Medial and final liquids (Trefry 1969:2-5; Blevins 1994:321)

\begin{tabular}{|c|c|c|c|}
\hline mere & 'many' & priso & 'hear! (DU IMP)' \\
\hline borumai & 'blood' & suara & 'one' \\
\hline if & 'cold' & piraLendi & 'warm' \\
\hline jaı_o & 'plant! (SG IMP)' & jalo $</$ jaL $+\mathrm{io} /$ & 'plant! (PL IMP)' \\
\hline biLe & 'broken' & gaLpuLa & 'hat' \\
\hline jaL & 'man' & & \\
\hline
\end{tabular}

As in Mongolian, initial glides (30) and vowel-initial words (31) are possible in Kuman (Trefry 1969:12-13). 
(30) Initial glides (Trefry 1969:2-3, Blevins 1994:321-4)

$\begin{array}{llll}\text { jaralka } & \text { 'I will plant' } & \text { jobuLo } & \text { 'bone-3SG' } \\ \text { juo } & \text { 'bring! (SG IMP)' } & \text { wam } & \text { 'fat' } \\ \text { winale } & \text { 'claw' } & \text { wagai } & \text { 'good' }\end{array}$

(31) Vowel-initial words (Trefry 1969:13-14)

$\begin{array}{llll}\text { i } & \text { 'this' } & \text { ir } & \text { 'cold' } \\ \text { olto } & \text { 'long' } & \text { amuL } & \text { 'pandanus' } \\ \text { amniLo } & \text { 'milk' } & & \end{array}$

Once again, it is the class of liquids that is excluded from initial-syllable onsets.

Two more examples of this pattern are the Australian languages Guugu Yimidhirr and Pitta-Pitta, which ban word-initial liquids while permitting other consonants in word-initial position (Dixon 1980: Ch. 6). (Unlike Mongolian and Kuman, however, these two languages do not allow onsetless syllables; see footnote 5.)

In Guugu Yimidhirr (Dixon 1980:162-3), there is an apical lateral [1] and two rhotics, the apico-alveolar trill $[\mathrm{r}]$ and the retroflex approximant $[\mathrm{l}]$. However, none of these liquids are possible in initial position-even though Guugu Yimidhirr, unlike some Australian languages, does allow the non-liquid apicals [d,n] in initial position. 
(32) Consonant inventory of Guugu Yimidhirr (Dixon 1980:162)

(Shaded segments are banned from word-initial position.)

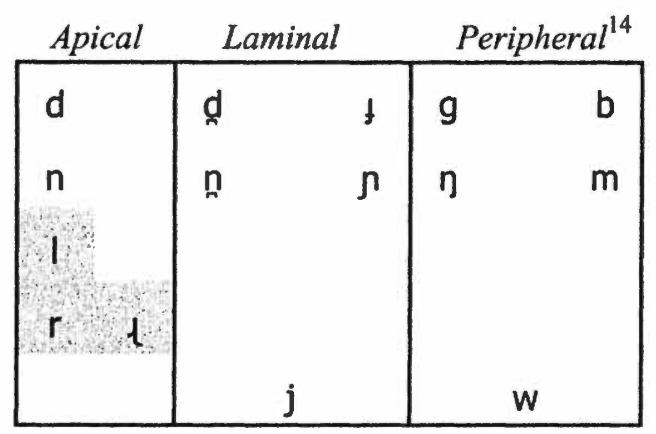

In Pitta-Pitta (Dixon 1980:160-161), all apico-alveolar consonants are banned from word-initial position (dashed box in (33)), so naturally there are no initial apico-alveolar liquids. However, this language has a number of additional liquids at other places of articulation, and none of those liquids are possible initial onsets either. That is, in addition to a place-based ban on initial apico-alveolars, Pitta-Pitta also has a general ban on initial liquids.

Consonant inventory of Pitta-Pitta (Dixon 1980:160)

(Shaded segments are banned from word-initial position.)

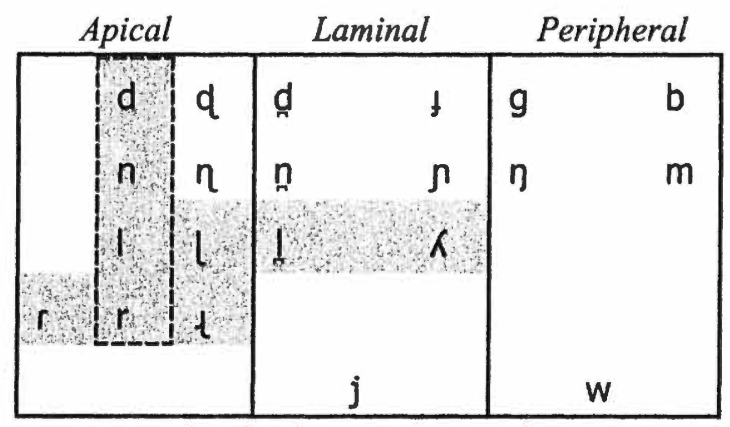

Thus, both Guugu Yimidhirr and Pitta-Pitta also exclude specifically liquids from the set of possible initial onsets.

14. Dixon (1980) groups labial and velar consonants together as "peripheral" consonants because they typically pattern as a class in Australian languages. 
These four languages that systematically ban liquid onsets in initial syllables-Mongolian, Kuman, Guugu Yimidhirr, and Pitta-Pitta-can be accounted for with a ranking in which the positional augmentation constraint $\left[{ }^{*} \mathrm{ONSET} / \mathrm{LAT}\right] / \sigma_{1}$ dominates at least one relevant faithfulness constraint. (Since $\left[{ }^{*} \mathrm{ONSET} / \mathrm{RHO}\right] / \sigma_{1}$ dominates [*ONSET/LAT] $/ \sigma_{1}$ universally, both rhotics and laterals are thus impossible onsets. ${ }^{15}$ ) If $\left[{ }^{*}\right.$ ONSET/RHO] $/ \sigma_{1}$ and $\left[{ }^{*}\right.$ ONSET/LAT $] / \sigma_{1}$ outrank at least one (relevant) faithfulness constraint, then the lowest ranked of such faithfulness constraints, $\mathbf{F}_{n}$, will be violated when necessary to avoid a liquid onset in the initial syllable. Depending on what faithfulness constraint stands in the place of $\mathbf{F}_{n}$ in a particular language, several strategies for avoiding initial liquids are possible.

If $\mathbf{F}_{n}$ is DEP-SEG, then epenthesis will occur to ensure that the input initial liquid is not an initial-syllable onset in the output (34). In \$4.2.1.2.1 above, Campidanian Sardinian was argued to be a language with this kind of ranking: vowels are epenthesized when otherwise initial onsets would be unacceptably high in sonority.

Initial liquid onset avoided by epenthesis

\begin{tabular}{|r||c|c|c|c|c|}
\hline /rana/ & $\begin{array}{c}{\left[{ }^{*} \text { ONS/ }\right.} \\
\mathrm{R}] / \sigma_{1}\end{array}$ & $\begin{array}{c}{\left[{ }^{*} \text { ONS/ }\right.} \\
\mathrm{L}] / \sigma_{1}\end{array}$ & $\begin{array}{c}\text { MAX- } \\
\text { SEG }\end{array}$ & IDENT[f] & $\begin{array}{c}\text { DEP- } \\
\text { SEG }\end{array}$ \\
\hline \hline a. rana & $* !$ & & & & \\
\hline b. erana & & & & & $*$ \\
\hline c. ana & & & $* !$ & & \\
\hline d. tana & & & & $* !$ & \\
\hline
\end{tabular}

If $\mathbf{F}_{n}$ is MAX-SEG, then initial liquid onsets will be deleted (35).

15. Of course, $\left[{ }^{*} \mathrm{ONSET} / \mathrm{GLI}\right] / \sigma_{1}$ universally outranks $\left[{ }^{*} \mathrm{ONSET} / \mathrm{RHO}\right] / \sigma_{1}$, since glides are higher in sonority than rhotics. But as noted above, the four languages discussed in this section do not prohibit word-initial glides. In $\S 4.2 .1 .2 .4$, a connection is drawn between the fact that a ban on liquid (or only rhotic) onsets does not always entail a ban on syllableinitial glides and the fact that syllable-initial glides are not always structural onsets, but may sometimes be onglides within the nucleus. 
(35) Initial liquid onset avoided by liquid deletion

\begin{tabular}{|c||c|c|c|c|c|}
\hline /rana/ & $\begin{array}{c}{\left[{ }^{*} \text { ONS/ }\right.} \\
\mathrm{R}] / \sigma_{1}\end{array}$ & $\begin{array}{c}{\left[{ }^{*} \text { ONS/ }\right.} \\
\mathrm{L}] / \sigma_{1}\end{array}$ & $\begin{array}{c}\text { DEP- } \\
\text { SEG }\end{array}$ & IDENT[f] & $\begin{array}{c}\text { MAX- } \\
\text { SEG }\end{array}$ \\
\hline \hline a. rana & $* !$ & & & & \\
\hline b. erana & & & $* !$ & & \\
\hline c. ana & & & & & $*$ \\
\hline d. tana & & & & $* !$ & \\
\hline
\end{tabular}

Finally, if $\mathbf{F}_{n}$ is IDENT[f] for some feature $f$, then the optimal output for a form with an initial liquid is one that has changed the $f$ specification for the liquid so that it surfaces as a non-liquid. This is the ranking that is relevant for Kuman [r], which according to Blevins (1994) surfaces as [t] in word-initial position (as well as preconsonantally).

(36) Initial liquid onset avoided by altering the liquid

\begin{tabular}{|c||c|c|c|c|c|}
\hline /rana/ & $\begin{array}{c}{\left[{ }^{*} \text { ONS/ }\right.} \\
\mathrm{R}] / \sigma_{1}\end{array}$ & $\begin{array}{c}{ }^{*} \mathrm{ONS} / \\
\mathrm{L}] / \sigma_{1}\end{array}$ & $\begin{array}{c}\text { DEP- } \\
\text { SEG }\end{array}$ & $\begin{array}{c}\text { MAX- } \\
\text { SEG }\end{array}$ & IDENT[f] \\
\hline \hline a. rana & $* !$ & & & & \\
\hline b. erana & & & $* !$ & & \\
\hline c. ana & & & & $* !$ & \\
\hline d. tana & & & & & $*$ \\
\hline
\end{tabular}

Although for some of the languages surveyed in this section it is not possible to tell precisely which repair strategy is chosen for avoiding initial liquid onsets, it is clear that in each language the positional augmentation constraints $\left[{ }^{*} \mathrm{ONSET} / \mathrm{RHO}\right] / \sigma_{1} \gg\left[{ }^{*} \mathrm{ONSET} / \mathrm{LAT}\right] / \sigma_{1}$ are ranked above at least one faithfulness constraint; initial liquid onsets do not surface. 


\subsection{Initial rhotic onsets banned in Mbabaram}

The third pattern of initial-syllable onset sonority restrictions to be discussed here is that found in Mbabaram (Australian; Dixon 1991). This pattern is related to both of the patterns just discussed. Like Sestu Campidanian (§4.2.1.2.1), Mbabaram bans rhotics from initial onset position while allowing laterals. But unlike Sestu, and like the four languages discussed in \$4.2.1.2.2, Mbabaram allows word-initial glides.

Mbabaram has three liquids in its consonant inventory: the apico-alveolar lateral [l], the 0alveolar tap or trill [ $\mathrm{r}$ ], and the retroflex [ $\mathrm{c}]$, "which may be a tap, a trill, or a rhotic continuant" (Dixon 1991:356). However, while the lateral may appear word-initially (37), both of the rhotics are banned from that position (Dixon 1991:359). ${ }^{16}$

Word-initial laterals in Mbabaram (Dixon 1991:398-401)

$\begin{array}{ll}\text { lim } & \text { 'good, fresh, satisfied' } \\ \text { lo:r I } & \text { 'whip-tail kangaroo' } \\ \text { libór } & \text { 'eye' } \\ \text { lugúl } & \text { 'long way' } \\ \text { lábi- } & \text { 'leave (something in a place)' } \\ \text { lángil } & \text { 'light' }\end{array}$

As noted, word-initial glides do appear in Mbabaram, but further discussion of this fact and its implications is postponed until §4.2.1.2.4.

16. One might ask whether there could be a confounding factor, unrelated to sonority differences, when languages ban initial rhotics but not initial laterals. Several kinds of rhotics, including taps and trills, occur preferentially between vowels, so there may be independent constraints unrelated to the *ONSET/X hierarchy that prevent these kinds of rhotics from surfacing in word-initial position. However, the fact that Mbabaram [ $r$ ] can be realized as a continuant in addition to its realization as a tap or trill, and yet is still banned from word-initial position, indicates that it really is ${ }^{*}[\mathrm{ONSET} / \mathrm{RHO}] / \sigma_{1}$, and not simply a ban on initial taps and trills, that is active in this language. 
(38) Word-initial glides in Mbabaram (Dixon 1991:398-401)

$\begin{array}{llll}\text { jł } & \text { 'I (subject)' } & \text { wirr } & \text { 'head hair' } \\ \text { ju } & \text { 'fish' } & \text { we } & \text { 'mouth, teeth' } \\ \text { ja-t } & \text { 'give-PAST' } & \text { wu: } & \text { 'mother' } \\ \text { jigír } & \text { 'itchy' } & \text { wo } & \text { 'west' } \\ \text { jargúl } & \text { 'woman' } & \text { walná } & \text { 'girl' } \\ & & \text { wurgún } & \text { 'boy' }\end{array}$

The fact that Mbabaram disallows initial rhotics shows that [*ONSET $/$ RHO]/ $\sigma_{1}$ outranks some faithfulness constraint (such as DEP-SEG, MAX-SEG, or IDENT[f]).

Initial rhotics banned in Mbabaram (hypothetical input)

\begin{tabular}{|c||c|c|c|c|}
\hline$/$ ramba/ & {$\left[{ }^{*} \mathrm{ONS}^{\prime} / \mathrm{RHO}\right] / \sigma_{1}$} & $\begin{array}{c}\text { DEP- } \\
\text { SEG }\end{array}$ & $\begin{array}{c}\text { MAX- } \\
\text { SEG }\end{array}$ & IDENT[f] \\
\hline \hline a. ramba & $* !$ & & & \\
\hline$(\bowtie)$ b. aramba & & $*$ & & \\
\hline$(\infty)$ c. amba & & & $*$ & \\
\hline$(\infty)$ d. damba & & & & $*$ \\
\hline
\end{tabular}

Again, there is not enough information to tell whether the actual output for this kind of input would be (39b), ${ }^{17}$ (39c), or (39d), but crucially, it is not (39a).

Laterals, unlike rhotics, are permissible initial onsets. Therefore, all relevant faithfulness constraints, such as DEP-SEG, MAX-SEG, and IDENT[f], represented here by FAITH, must dominate [*ONSET/LAT]/ $\sigma_{1}$.

17. Mbabaram is an "initial-dropping" language (Dixon 1991:351), which gives it an atypical phonological system for an Australian language. For example, most Australian languages require all syllables to have onsets, but Mbabaram tolerates the word-initial vowel [a]. (Arrernte, discussed in Chapter 3, is another initial-dropping language, but the two languages are geographically separated and, along with still other languages, are argued by Dixon (1980) to have undergone similar diachronic changes independently.) 


\begin{tabular}{|r||c|c|c|}
\hline /langil/ & {$\left[{ }^{*}\right.$ ONS/RHo $] / \sigma_{1}$} & FAITH & {$\left[{ }^{*}\right.$ ONS/LAT $] / \sigma_{1}$} \\
\hline \hline a. langil & & & $*$ \\
\hline b. alangil & & $* !$ & \\
\hline c. angil & & $* !$ & \\
\hline d. dangil & & $* !$ & \\
\hline
\end{tabular}

The above analysis of Mbabaram initial-syllable onsets concludes the set of case studies presented to exemplify constraints from the [ $\left.{ }^{*} \mathrm{ONSET} / \mathrm{X}\right] / \sigma_{1}$ subhierarchy. Before closing the discussion of $\mathbf{M} / \sigma_{1}$ constraints (see $\S 4.2 .1 .3$ ), however, this section addresses one final topic: the conclusions that can be drawn on the basis of these case studies about the formulation of [*ONSET/X] constraints - in particular, how an additional possible syllabic position for pre-peak glides can allow a syllable to begin with a glide without violating [ ${ }^{*}$ ONSET/GLI]

\subsection{Implications for syllable structure and onset-related constraints}

This section discusses certain implications that the results of the [ $\left.{ }^{*} \mathrm{ONSET} / \mathrm{X}\right] / \sigma_{1}$ case studies have for constraint formulations and for subsyllabic structure. The crucial question is the structural position of syllable-initial glides. A distinction has been made in the literature between glides that are true consonantal onsets, and those that precede the syllable peak but are included within the nucleus (Kaye \& Lowenstamm 1984; Davis \& Hammond 1995; Harris \& Kaisse 1999). The proposal developed here is that $\left[{ }^{*} \mathrm{ONSET} / \mathrm{GLI}\right]\left(/ \sigma_{1}\right)$ is violated by true onset glides, but not by nuclear onglides; this allows glides to "escape" onset sonority restrictions in certain languages.

As seen above, languages like Mongolian, Kuman, Guugu Yimidhirr, and PittaPitta avoid liquid onsets in initial syllables, but they allow words to begin with glides. The avoidance of initial liquids was analyzed by means of a ranking in which the positional augmentation constraints [*ONSET/RHo] $/ \sigma_{1} \gg\left[{ }^{*} \mathrm{ONSET} / \mathrm{LAT}\right] / \sigma_{1}$ dominate at least one relevant faithfulness constraint. As a result of this ranking, any initial liquid in the input will fail to surface as such-depending on the ranking among the faithfulness constraints, the liquid will be deleted or changed into a nonliquid segment, or a prothetic vowel will appear. 
However, the constraints [ $\left.{ }^{*} \mathrm{ONSET} / \mathrm{RHO}\right] / \sigma_{1}>\left[{ }^{*} \mathrm{ONSET} / \mathrm{LAT}\right] / \sigma_{1}$ do not exist in isolation. They are part of the $\left[{ }^{*} \mathrm{ONSET} / \mathrm{X}\right] / \sigma_{1}$ constraint subhierarchy, whose component constraints are in a universally fixed ranking that is derived from the segmental sonority scale (\$2.3.2.3.3). In particular, the constraint [ $\left.{ }^{*} \mathrm{ONSET} / \mathrm{GLI}\right] / \sigma_{1}$ always dominates $\left[{ }^{*} \mathrm{ONSET} / \mathrm{RHO}\right] / \sigma_{1}$. By transitivity of ranking, this entails that whenever $\left[{ }^{*}\right.$ ONSET $/$ RHO $] / \sigma_{1}$ dominates some faithfulness constraint $\mathbf{F}$, $\left[{ }^{*} \mathrm{ONSET} / \mathrm{GLI}\right] / \sigma_{1}$ also dominates $\mathbf{F}$. So if a language avoids initial liquid onsets through vowel prothesis (meaning that DEP-SEG is dominated by $\left.\left[{ }^{*} \mathrm{ONSET} / \mathrm{RHO}\right] / \sigma_{1} \gg\left[{ }^{*} \mathrm{ONSET} / \mathrm{LAT}\right] / \sigma_{1}\right)$, the prediction is made that initial glide onsets will be avoided through prothesis as well. ${ }^{18}$

A ban on liquids entails a ban on glides

(a)

\begin{tabular}{|c||c|c|c|}
\hline$/ \mathrm{la} /$ & {$\left[{ }^{*}\right.$ ONS/RHO $] / \sigma_{1}$} & {$\left[{ }^{*}\right.$ ONS/LAT $] / \sigma_{1}$} & $\begin{array}{c}\text { DEP- } \\
\text { SEG }\end{array}$ \\
\hline \hline a. la & & $* !$ & \\
\hline & & & $*$ \\
\hline
\end{tabular}

18. Another situation in which a language would satisfy $\left[{ }^{*} \mathrm{ONSET} / \mathrm{RHO}\right] / \sigma_{1} \gg$ $\left[{ }^{*}\right.$ ONSET $/$ LAT $] / \sigma_{1}$ while nevertheless violating $\left[{ }^{*} \mathrm{ONSET} / \mathrm{GLI}\right] / \sigma_{1}$ might arise if the lowranking faithfulness constraint whose violation was compelled by $\left[{ }^{*} \mathrm{ONSET} / \mathrm{RHO}\right] / \sigma_{1} \gg$ $\left[{ }^{*}\right.$ ONSET $\left./ \mathrm{LAT}\right] / \sigma_{1}$ were a faithfulness constraint for a feature that was crucial to the identity of a liquid but not to that of a glide. For example, imagine a language in which underlying word-initial liquids are avoided because they surface as [n] (a pattern that is actually seen in Korean, but for all (non-ambisyllabic) syllable onsets, not just word-initial onsets). This would indicate that featural faithfulness constraints such as IDENT[nasal] and IDENT[lateral] are ranked below $\left[{ }^{*} \mathrm{ONSET} / \mathrm{RHO}\right] / \sigma_{1}>\left[{ }^{*} \mathrm{ONSET} / \mathrm{LAT}\right] / \sigma_{1}$. If IDENT[consonantal] (as well as MAX-SEG and DEP-SEG) outranks [ $\left.{ }^{*} \mathrm{ONSET} / \mathrm{GLI}\right] / \sigma_{1}$, initial glides will surface unchanged even though initial liquids are altered, because glides cannot become lower in sonority without changing from [-cons] to [+cons], but liquids are already [+cons] and will remain so even if they change into lower-sonority segments.

However, the problem of initial glides in languages that ban only initial liquids is a more general problem that requires a general explanation; feature alteration is not always the way that languages choose to avoid initial liquid onsets. For example, the Iglesias dialect of Campidanian Sardinian avoids initial liquid onsets (as does Sestu) but allows initial glides (unlike Sestu) (Bolognesi 1998:44). Crucially, initial liquid onsets are avoided through prothesis. There is no explanation intrinsic to the featural composition of glides as to why prothesis would be blocked before glides and not before liquids. 
(b)

\begin{tabular}{|c|c|c|c|c|}
\hline /ja/ & $\begin{array}{l}{\left[{ }^{*} \text { ONS/ }\right.} \\
\text { GLI] } / \sigma_{1}\end{array}$ & $\begin{array}{r}{\left[{ }^{*} \mathrm{ONS} /\right.} \\
\mathrm{RHO}] / \sigma_{1}\end{array}$ & $\begin{array}{l}\text { [*ONS/ } \\
\text { LAT]/的 }\end{array}$ & $\begin{array}{l}\text { DEP- } \\
\text { SEG }\end{array}$ \\
\hline a. ja & $* !$ & & & in \\
\hline b. eja & & & & * \\
\hline
\end{tabular}

How, then, is it possible for a language to avoid initial liquid onsets while nevertheless tolerating initial glides? This apparent contradiction can be resolved if it is recognized that not all syllable-initial glides are truly onsets, as in (42a). Some such glides are actually part of the nucleus; in other words, they are dominated by a mora, as shown in (42b). (Glides that form part of the nucleus but precede the peak, as in (42b), will be called nuclear onglides.)

(a) True onset

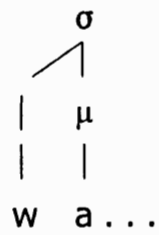

(b) Nuclear onglide

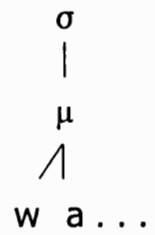

This structural distinction is possible to maintain under a version of moraic theory in which onset consonants are directly affiliated with the syllable node (Hayes 1989; McCarthy \& Prince 1986). Failure to be dominated by a mora then becomes the structural diagnostic for a true onset; as seen below, the structural difference between the glides in (42a) and (42b) can be exploited in the formulation of constraints.

Supporting evidence for the claim that languages distinguish between true consonantal onset glides and nuclear onglides can be found in French (Kaye \& Lowenstamm 1984), Spanish (Harris \& Kaisse 1999; see also Harris 1983, Hualde 1989), Slovak (Rubach 1998; Harris \& Kaisse 1999), and American English (Davis \& Hammond 1995).

Kaye \& Lowenstamm (1984) propose that glides in French occupy true onset positions in some words, but are nuclear onglides in other words. Their evidence comes mainly from the observation that glides in native words act like vowels, whereas those in loanwords act like consonants, but they offer additional support for their claim from facts about syllable structure and co-occurrence restrictions.

There are several well-known phonological processes that occur at the boundary beteen two words in French and are conditioned by the vowel/consonant status of the initial segment of the second word. Vowel (schwa) elision takes place 
before a V-initial word, but not before a C-initial word (43a); liaison ${ }^{19}$ takes place before a V-initial word, whereas a final consonant is (under certain conditions) deleted before a $\mathrm{C}$-initial word (43b); and suppletive forms of certain determiners are conditioned by $\mathrm{V}$-initial versus $\mathrm{C}$-initial words (43c).

(43) Phonological processes sensitive to word-initial V/C distinction in French (Kaye \& Lowenstamm 1984:135)
pre- $V$
pre- $C$
(a) V elision
$\checkmark \quad$ [IØ etydjã]
'the student'
NA [la bato]
'the boat'
(b) Liaison
[pətiț etydjã]
'small student'
NA [patiø bato]
'small boat'
(c) Suppletion
[şt etydjã]
- C
[so bato]
'this boat'

Words with initial glides fall into two groups with respect to the V/C-sensitive processes exemplified in (43), as shown in (44). Glide-initial native words pattern with V-initial words, and glide-initial (recent) loanwords pattern with C-initial words.

Glide-initial words in French

(Clements \& Keyser 1983; Kaye \& Lowenstamm 1984)

Native
(a) V elision

[!Ø jatys]

'the hiatus'

[lØ wazo]

'the bird'
Loanword

$\begin{array}{ll}\text { NA } & {[\underline{\text { la jogi }]}} \\ & \text { 'the yogi' } \\ & {[\underline{\text { la } w i s k i]}} \\ & \text { 'the whisky' }\end{array}$

19. For the purposes of the present discussion, liaison may be regarded as the resyllabification of a word-final consonant, but see, e.g., Schane (1968), Selkirk (1974), and Clements \& Keyser (1983) for more detailed discussion of this complex process. 
Native

(b) Liaison

$\checkmark$

$[\mid \varepsilon \underline{z} \mathrm{j} \varnothing]$

'the eyes'

[patit wazo]

'small bird'

(c) Suppletion [set jatys]

'this hiatus'

[set wazo]

'this bird'
Loanword

\begin{tabular}{|c|c|}
\hline NA & $\begin{array}{l}\text { [l\&Ø jogi] } \\
\text { 'the yogis' } \\
\text { [potiØ wiski] } \\
\text { 'small whisky' }\end{array}$ \\
\hline${ }_{-} C$ & $\begin{array}{l}\text { [so jogi] } \\
\text { 'this yogi' } \\
\text { [so wiski] } \\
\text { 'this whisky' }\end{array}$ \\
\hline
\end{tabular}

It has been proposed (Milner 1973, Clements \& Keyser 1983, Kaye \& Lowenstamm 1984) that the difference between the initial glides in native words like oiseau [wazo] 'bird' and those in loanwords like whisky [wiski] 'whisky' is that the glides are "vocalic" in the former case but "consonantal" in the latter. Kaye \& Lowenstamm (1984) further propose that this distinction is actually the result of two different structural representations for the two classes of glides: the glide in whisky is a true onset, while that in oiseau is part of a complex nucleus, forming a rising diphthong - what has been termed here a nuclear onglide.

Structural representations for glides in French (after Kaye \& Lowenstamm 1984:135)

(a) Glide as onset

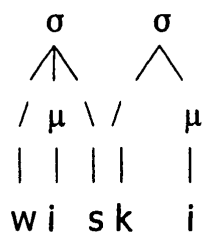

(b) Glide as nuclear onglide

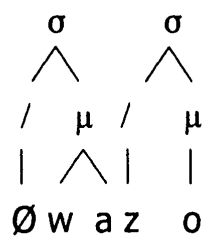

That is, the word whisky has an element in the onset position, so it behaves like other consonant-initial words. However, oiseau has no true onset consonant, so it participates in processes such as vowel elision and liaison just as unambiguously vowel-initial words do.

Kaye \& Lowenstamm (1984:135-9) present additional support for the distinction that they draw between the two types of initial glides. They note that 
words of the oiseau type, which trigger pre-V phonological processes, show more stringent co-occurrence restrictions between the glide and the following vowel than do words of the whisky type; they interpret these facts to mean that restrictions hold between elements of a complex nucleus that do not hold between an onset glide and a nuclear vowel. Furthermore, the same restricted set of glide+vowel pairs that trigger pre- $\mathrm{V}$ phonological processes are also involved in various (synchronic or diachronic) alternations with single vowels, as in verra [vera] 'see-3SG.FUT' versus voit [vwa] 'see-3SG.PRES'; these alternations are at least suggestive that the glide+vowel sequences in question are complex nuclei (in the alternating words) rather than onset+nucleus sequences. Finally, Kaye \& Lowenstamm (1984) show that possible word-initial stop+liquid+glide $+\mathrm{V}$ sequences correlate with the possible glide $+\mathrm{V}$ sequences in oiseau-type words. For example, trois [trwa] 'three' is permitted, but trouer /tru+e/ 'to bore a hole in' is [tru.we], not *[trwe] (cf. louer $/ l u+e /$ 'to rent,' which is [lwe]). Crucially, the [wa] in trois is one of the small number of permitted glide+vowel sequences seen in the oiseau-type words, that is, one of the set of possible complex nuclei. However, [we] is not a possible complex nucleus, so in this case the [w] must be syllabified into the onset. A stop+liquid sequence may precede a complex nucleus ([tr'wa]), but it may not form a triply complex onset with a glide $(*[\operatorname{trw} \cdot e])$.

Thus, Kaye \& Lowenstamm (1984) have proposed that two distinct syllabic positions for glides must be recognized in French, on the basis of diagnostics for onset versus nuclear status as well as co-occurrence restrictions on complex onsets and complex nuclei. Crucially, the difference between monosyllabic [trwa], with nuclear onglide $[w]$, and disyllabic [tru.we], with onset [w] $\left({ }^{*}[\right.$ trwe]), shows that the difference between the two kinds of glides is not simply a loanword/native word difference, but is relevant within the phonology of native words as well. ${ }^{20}$

Arguments in favor of a distinction between onset and rimal syllabification of pre-peak glides have also been made for Spanish (Harris \& Kaisse 1999; see also Harris 1983, Hualde 1989) and Slovak (Rubach 1998; Harris \& Kaisse 1999). In these languages, facts about syllable weight and (for Spanish) possible syllable

20. Systematic differences between the phonotactics of native morphemes and etymological loanwords have been given several different treatments in OT, including lexical stratum-specific rankings (Itô \& Mester 1995), stratum-specific constraints (Fukazawa, Kitahara, \& Ota 1998), and the use of underlyingly specified versus unspecified properties (Inkelas, Orgun, \& Zoll 1997). A detailed analysis of how the constraint ranking differs between French native morphemes and loanwords, resulting in their respective syllabifications for glides, will not be pursued here. What is important for the current discussion is simply that there is evidence for a difference between onset glides and nuclear onglides in the phonology of French. 
shapes and glide fortition patterns show that a pre-peak glide is parsed as part of the rime unless there is nothing else to be parsed as a true onset, in which case the glide fills that slot.

Harris \& Kaisse (1999) present several pieces of evidence concerning the syllabification of glides in Spanish. First, they show that a CGV syllable is heavy, but a GV syllable is light. Antepenultimate stress is possible, as a lexically marked option, in Spanish nouns and adjectives, but this is blocked in words that have a heavy penult or a heavy ultima. Crucially, in all dialects of Spanish, antepenultimate stress is also impossible if the penult or ultima contains a CGV syllable (Harris \& Kaisse 1999:149). Therefore, CGV syllables behave like heavy syllables.

CGV syllables are heavy (Harris \& Kaisse 1999:149)

(a) Final .CGV.

$\begin{array}{lll}\text { cal.ví.c[j]e } & \text { 'baldness' } & \text { but * 'CVX.CV.CGV\# } \\ \text { au.dá.c[j]a } & \text { 'audacity' } & \\ \text { ne.gó.c[j]o } & \text { 'business' } & \end{array}$

(b) Penultimate .CGV.

$\begin{array}{lll}\text { tra.v[j]é.so } & \text { 'mischievous' } & \text { but }{ }^{*} \text { 'CVX.CGV.CV\# } \\ \text { me.d[j]ó.cre } & \text { 'mediocre' } & \\ \text { a.c[j]á.go } & \text { 'ominous' }\end{array}$

In contrast, GV syllables do not pattern as heavy syllables; a GV penult still allows antepenultimate stress.

(47) GV syllables are light (Harris \& Kaisse 1999:148) ${ }^{21}$

o.no.ma.to.pé.[j]i.co 'onomatopoeic'

Plé.[j]a.des 'Pleiades'

Sá.[j]a.go (surname)

Harris \& Kaisse conclude from these facts that a glide contributes to weight if and only if it is not the initial segment in the syllable.

21. Syllable-initial glides, as in these examples, are subject to glide hardening in many dialects of Spanish. See below. 
Another piece of evidence that syllable-initial glides in Spanish are syllabified differently from glides preceded by other consonants comes from the inventory of possible syllable shapes. According to Harris \& Kaisse, *CGVGs and *CGVCs syllables are impossible, even though substrings of those sequences are possible syllables (48a). Furthermore, similar syllables with a liquid $\mathrm{R}$ in place of the prepeak glide are possible (48b), and another five-segment syllable shape CRGVC is possible (48c), so (unsurprisingly) the relevant restriction has nothing to do with segment count.

Possible syllable shapes (Harris \& Kaisse 1999:126)
(a) CGVG [bwej]
'ox'
but not *CGVGs

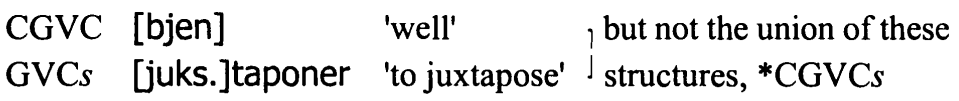
cf. (b) CRVGs [klaws.] tro 'cloister'
CRVCs [trans.]formar 'to transform'
(c) CRGVC pu[.drjen.]do 'rotting'
but not *CRGVGs, *CRGVCs

The reason for the impossibility of C(R)GVXs syllables, according to Harris \& Kaisse, is that the glide in these syllables is part of the rime, unlike the liquid in a $\mathrm{CR}(\mathrm{G}) \mathrm{VX}(s)$ syllable, which is part of a complex onset. As shown by the stress facts discussed above, glides are weight-bearing when preceded by another consonant, but non-weight-bearing when in absolute syllable-initial position. If Spanish syllables are maximally bimoraic (with an optional weightless final element), this would rule out C(R)GVXs - all the underlined elements would be weight-bearing, and so this syllable would be too large. ${ }^{22}$

Thus, there are two pieces of evidence that glides in Spanish are weight-bearing when they are preceded by an onset consonant. First, CGV syllables, but not GV syllables, pattern as heavy syllables for the purposes of stress assignment. Second, a glide is counted toward the maximum syllable size limit when it is preceded by an onset consonant, but not when it is initial in the syllable. Under the standard

22. Harris \& Kaisse (1999:129) are not working within moraic theory, so they do not describe this limit in terms of moras; they state it as a limit of three segments in the rime, or a restriction that the rime may branch at most twice. 
assumption that a weight-bearing element must be part of the rime, this shows that a pre-peak glide preceded by another consonant is part of the rime. The fact that a syllable-initial glide is not weight-bearing moreover suggests that a glide in this environment is not part of the rime, but this fact alone is not necessarily conclusive, since not all rimal segments contribute to syllable weight.

Stronger evidence that a syllable-initial glide (with no preceding onset consonant) is not part of the rime comes from facts about glide hardening. In many dialects of Spanish, syllable-initial [j] in this position may become something like [3] or [j], and [w] may become something like $\left[\mathrm{g}^{\mathrm{w}}\right] .^{23}$ This kind of sonorityreduction effect would be unexpected in a segment belonging to the rime, but the preference for low-sonority onsets is quite well established (formally, glide hardening would thus be seen as a response to (general) *ONSET/GLIDE).

Therefore, translating Harris \& Kaisse (1999)'s proposal into moraic-theory representations, pre-peak glides in Spanish have one of the following two structural positions, depending on whether or not an onset consonant precedes.

(a) True onset

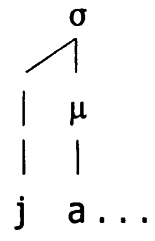

(b) Nuclear onglide $\sigma$

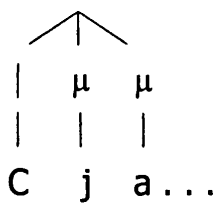

Crucially, in Spanish, a rimal pre-peak glide as in (49b) is associated with its own mora; this is why it contributes to weight. This need not be true for all languages, however. Even offglides, as in [ai], are not weight-bearing in all cases, so there should be quantity-sensitive languages in which nuclear onglides do not contribute to syllable weight, indicating that they are adjoined to the mora associated with the syllable peak as shown for French in (45) above. (Note, however, that since French

23. Harris \& Kaisse (1999) use the failure of glides to harden when another onset consonant is present as an additional argument that such glides are not true onsets. However, this particular argument does not hold up in the context of the analysis under development here. Recall from the discussion of *\#r onsets versus \# $\mathrm{Cr}$ clusters in Campidanian Sardinian above that $\left[{ }^{*} \mathrm{ONSET} / \mathrm{GLI}\right] / \sigma_{1}$ would never be violated by a glide in a \#CGV syllable-even if it were syllabified as a true consonantal onset-because the glide is not the leftmost segment in the syllable. Thus, failure to harden in the context of a preceding onset consonant does not necessarily prove that the glide in question is not also syllabified as an onset. (However, the evidence for rime status based on syllable weight that has just been described seems quite convincing.) 
is not quantity-sensitive, a structure with a shared mora cannot be definitively proven in the case of French specifically.) Incidentally, the term "nuclear onglide" is still appropriate in the case of Spanish, even though the vocoid in question heads its own mora, because the syllable peak is the following non-high vocoid.

Finally, Davis \& Hammond (1995) argue that both of these structural possibilities for pre-peak glides are found in American English. They account for the fact that $\mathrm{V}$ is nearly unrestricted in $j \mathrm{~V}, w \mathrm{~V}$, and $\mathrm{C} w \mathrm{~V}$ syllables, but restricted to $[\mathrm{u}]$ in $\mathrm{Cj} \mathrm{V}$ syllables, by proposing that $[\mathrm{j}]$ is a nuclear onglide when a preceding tautosyllabic consonant is present (like glides in Spanish and Slovak), but syllableinitial $[\mathrm{j}]$, and $[\mathrm{w}]$ in all cases, are true onsets.

In summary, it has been shown that more than one syllabic position is available for glides: they may be true consonantal onsets, or they may be part of the rime as nuclear onglides to the syllable peak. The choice of how to syllabify glides is made on a language-specific basis or even, as in French, Spanish, Slovak, and English, in a way that is dependent on the lexical or phonological context of the glides.

Since neither option for the syllabification of glides is universally avoided, there must be separate markedness constraints that prefer each of the structures over the other. Whether a language chooses to syllabify glides as onsets or as nuclear onglides will then depend on the relative ranking of these constraints.

First, there must be a constraint that penalizes true onset glides but not nuclear onglides. For this, we can make use of the familiar constraint *ONSET/GLI (in its general version as shown here, or in position-specific versions where applicable).

$$
\text { *ONSET/GLI For all syllables } x,|a|<\mathrm{GLI}
$$

where $a$ is the leftmost pre-moraic segment dominated by $x$ $|y|$ is the sonority of segment $y$

Now the consequences of the phrasing 'leftmost pre-moraic segment' in the formulation of this constraint become clear-a nuclear onglide, which is dominated by a mora, does not violate this constraint as it is given in (50). *ONSET/GLI refers to the onset proper, not just to any non-peak element at the left edge of the syllable.

In addition, there must be a constraint that penalizes nuclear onglides but not true onset glides. One possibility, following Rosenthall (1994), is *BRANCH- $\mu$, which prohibits two vowels from sharing a single mora. ${ }^{24}$

24. As seen above, Harris \& Kaisse (1999) demonstrate that, in Spanish (and Slovak), nuclear onglides are themselves weight-bearing vocoids. For languages of this type, the glide is apparently associated with its own mora (see (49b)), so it will not violate *BRANCH- $\mu$. Another constraint is therefore needed to rule out the structure in (49b), to account for 
(51) Constraint against nuclear onglides (after Rosenthall 1994:21)

${ }^{* \text { BRANCH- } \mu} \stackrel{*_{\mu}^{\mu}}{\mathrm{V}_{\mathrm{i}} \mathrm{V}_{\mathrm{j}}}$

A language that ranks *BRANCH- $\mu$ lower than *ONSET/GLI will syllabify glides as nuclear onglides (as long as *BRANCH- $\mu$ is also ranked below other relevant constraints, such as IDENT[f] constraints to prevent a glide from being changed into some other kind of segment, or MAX-SEG and DEP-SEG to prevent the deletion of the glide or the epenthesis of another segment respectively).

Languages with nuclear onglides

\begin{tabular}{|c|c|c|c|}
\hline /wa/ & *ONS/GLI & IDENT[f] & *BRANCH- $\mu$ \\
\hline a. $\quad$ w & $* !$ & & \\
\hline$\bigwedge_{1}^{\alpha}$ & & & 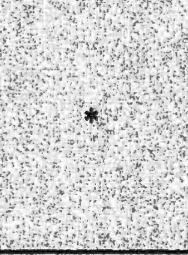 \\
\hline c. ba & & *! & \\
\hline
\end{tabular}

A language in which ${ }^{*}$ ONSET/GLI is lowest ranked will syllabify glides as true onsets.

languages (or environments within a language) that avoid moraic nuclear onglides in addition to those that share a mora with the syllable peak. One plausible option would be an alignment constraint that penalizes any mora intervening between the syllable peak (the head mora) and the left edge of the syllable. For simplicity of exposition, the rest of the discussion will refer only to *BRANCH- $\mu$, but this should be understood where necessary to represent all the constraints that rule out nuclear onglides, whether weight-bearing or not. 
(53) Languages with true onset glides

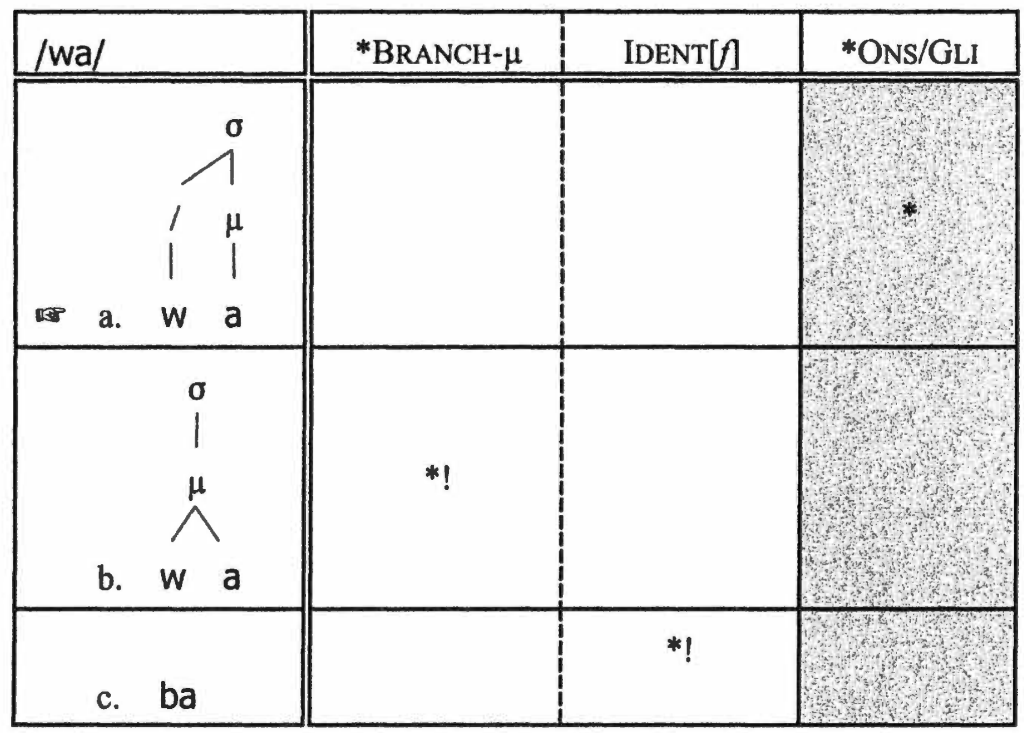

In this way, the structure that a language chooses for the syllabification of pre-peak glides depends on the relative ranking of ${ }^{*}$ ONSET/GLI, ${ }^{*}$ BRANCH- $\mu$, and other relevant constraints such as IDENT[f]. ${ }^{25}$

Now, given that pre-peak glides can be syllabified as part of the rime rather than as part of the onset proper, there is an explanation for why glides in some languages are able to "escape" sonority restrictions that hold for onsets otherwise. In languages such as Mongolian, Kuman, Guugu Yimidhirr, and Pitta-Pitta, where an avoidance of initial liquid onsets does not entail an avoidance of word-initial glides, *BRANCH- $\mu$ is apparently ranked low enough to allow pre-peak glides to be syllabified into the rime, avoiding violations of $\left[{ }^{*} \mathrm{ONSET} / \mathrm{GLI}\right] / \sigma_{1}$ while still maintaining segmental and featural faithfulness to word-initial glides (54).

25. Of course, natural languages are likely to have rankings more complicated than the illustrative rankings shown here. In Spanish, for example, a glide+V sequence is syllabified as onset+nucleus when no other onset consonant is present, but as a complex nucleus when there is a preceding onset consonant. This suggests that *BRANCH- $\mu$ (and the head-mora alignment constraint; see footnote 24) dominates *ONSET/GLI, such that onset syllabification of glides is preferred to rimal, but *COMPLEXONSET dominates the anti-onglide constraints, forcing glides into the rime when other onset consonants are present. (ONSET is satisfied by either true onset glides or nuclear onglides, so it can have no effect on glide syllabification; see below.) 
Languages that ban word-initial liquids but not word-initial glides ${ }^{26}$

(a) $\left[{ }^{*} \mathrm{ONS} / \mathrm{GLI}\right] / \sigma_{1} \gg\left[{ }^{*} \mathrm{ONS} / \mathrm{RHO}\right] / \sigma_{1} \gg\left[{ }^{*} \mathrm{ONS} / \mathrm{LAT}\right] / \sigma_{1} \gg \mathbf{F}$ (initial true-onset glides avoided; initial liquid onsets avoided)

(b) $\left[{ }^{*} \mathrm{ONS} / \mathrm{GLI}\right] / \sigma_{1} \gg \mathbf{F} \gg{ }^{*}$ BRANCH $-\mu$

(initial true-onset glides avoided by syllabification as nuclear onglides, rather than by segmental or featural unfaithfulness)

At this time, no independent evidence is available to confirm the proposal that the Mongolian-type languages syllabify word-initial glides as nuclear onglides. However, the above discussion of glides in French and Spanish has shown that the nuclear onglide position must be potentially available for pre-peak glides-even for absolute word-initial glides, as seen in French. If word-initial glides in the Mongolian-type languages are analyzed as nuclear onglides, then the fact that surface word-initial glides appear does not invalidate the use of [ $\left.{ }^{*} \mathrm{ONSET} / \mathrm{RHO}\right] / \sigma_{1}$ and/or [ ${ }^{*}$ ONSET/LAT] $/ \sigma_{1}$ to account for the absence of word-initial liquids. As a result, the ban on word-initial liquids can in all cases be seen as the work of positional versions of independently motivated onset-sonority markedness constraints.

On the other hand, languages that have anti-onglide constraints like *BRANCH$\mu$ ranked above some relevant faithfulness constraint are predicted to disallow nuclear onglides. Glides cannot escape into the nucleus to avoid onset sonority restrictions in these languages, so if initial onset liquids are banned, then initial glide onsets are banned as well (55).

(55) Languages that ban word-initial liquids and word-initial glides

(a) $\left[{ }^{*} \mathrm{ONS} / \mathrm{GLI}\right] / \sigma_{1} \gg\left[{ }^{*} \mathrm{ONS} / \mathrm{RHO}\right] / \sigma_{1} \gg\left[{ }^{*} \mathrm{ONS} / \mathrm{LAT}\right] / \sigma_{1} \gg \mathbf{F}$ (initial true-onset glides avoided; initial liquid onsets avoided)

26. When positional versions like $\left[{ }^{*} \mathrm{ONSET} / \mathrm{GLI}\right] / \sigma_{1}$ are included, a slightly more complex prediction is made: languages with the ranking [*ONSET/GLI]/str $>{ }^{*}$ BRANCH- $\mu \gg$ ${ }^{*}$ ONSET/GLI, $\mathbf{F}$ will have true onset glides in most cases $\left({ }^{*}\right.$ BRANCH- $\mu>>*^{*}$ ONSET/GLI, F), but nuclear onglides in the relevant strong position $\left(\left[{ }^{*} \mathrm{ONSET} / \mathrm{GLI}\right] /\right.$ str $>{ }^{*}$ BRANCH- $\left.\mu, \mathbf{F}\right)$ where true structural onsets must meet stricter sonority requirements. In practice, it may be empirically difficult to confirm that such a language exists, but it does not seem implausible. 
(b) $\left[{ }^{*} \mathrm{ONS} / \mathrm{GLI}\right] / \sigma_{1},{ }^{*} \mathrm{BRANCH}-\mu>\mathbf{F}$

(initial true-onset glides avoided by segmental or featural unfaithfulness, not by syllabification as nuclear onglides)

An example of a language that bans word-initial glides as well as liquids (specifically rhotics, in this case) is Sestu Campidanian. Some additional support for a relatively high rank for ${ }^{*}$ BRANCH- $\mu$ in Sestu can be seen in a property of this dialect reported in \$4.2.1.2.1 above: Sestu, unlike other dialects of Campidanian Sardinian, lacks CGV sequences altogether. Since pre-peak glides can potentially be syllabified either as true onsets or as nuclear onglides, the absence of CGV structures means that both of the following structures are banned in Sestu.

$$
\text { Possible syllabification of CGV syllables }
$$
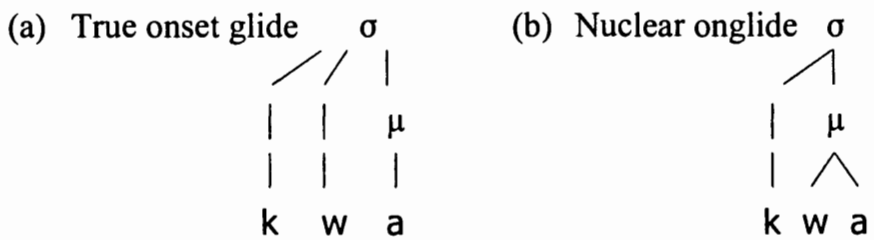

Since true onset glides and nuclear onglides are structurally distinct, the two syllabifications in (56) must be independently excluded. The ranking needed to ban (56a) is tangential to this discussion and will not be further investigated here ${ }^{27}$ what is crucial is that, if CGV syllables are never attested, then in addition to whatever ranking excludes (56a), ${ }^{*}$ BRANCH- $\mu$ must also be ranked high enough to exclude (56b). It is instructive to compare Sestu with the nearby Iglesias dialect of Campidanian Sardinian. Iglesias excludes word-initial liquids but not word-initial glides, which indicates that the word-initial glide in a form like [jaja] 'grandmother' (Bolognesi 1998:44) is a nuclear onglide. Iglesias is therefore predicted to permit the structure in (56b). In fact, Iglesias does allow CGV syllables, as in [kwad:u] 'horse' (Bolognesi 1998:24).

27. This is actually quite an interesting problem. It is not *COMPLEXONSET that rules out (56a) in Sestu, because complex onsets involving non-glides, as in tronu 'thunder,' do occur. One approach might be to make use of a constraint requiring glides (and other vocalic elements) to be moraic-perhaps as part of yet another sonority-related constraint subhierarchy - but the broader consequences of positing such a constraint family, and its interaction with other constraints in the system, require further investigation. 
In summary, a language that bans (initial) onset liquids, but not (initial) onset glides, does not serve as a counterexample to the universally ranked ${ }^{*} \mathrm{ONSET} / \mathrm{X}\left(/ \sigma_{1}\right)$ subhierarchy, because glides need not be syllabified as true onsets. Given the appropriate constraint ranking, a language can choose to syllabify glides as nuclear onglides instead.

\subsubsection{Summary: Initial-syllable augmentation}

Arapaho and Guhang Ifugao, discussed in $\$ 4.2 .1 .1$, provide evidence for the existence of a version of ONSET that is relativized to the strong position initial syllable - these languages allow onsetless syllables generally, but specifically require onsets in initial syllables.

The languages examined in $\S 4.2 .1 .2$ all provide evidence for an initial-syllablespecific version of the *ONSET/X subhierarchy, because they are languages in which high-sonority onsets are banned in initial syllables, but permitted in other syllables. In Mongolian, Kuman, Guugu Yimidhirr, and Pitta-Pitta, the first three constraints in the subhierarchy, $\left[{ }^{*} \mathrm{ONSET} / \mathrm{GLI}\right] / \sigma_{1} \gg\left[{ }^{*} \mathrm{ONSET} / \mathrm{RHO}\right] / \sigma_{1} \gg\left[{ }^{*} \mathrm{ONSET} / \mathrm{LAT}\right] / \sigma_{1}$, outrank the conflicting faithfulness constraints; as a result, glides and all liquids are banned from the true onset position in initial syllables (although glides do appear word-initially in these four languages, since they have an alternative possible syllabification as nuclear onglides). Mbabaram and Sestu Campidanian have only the first two of these constraints ranked above the conflicting faithfulness constraints, so they ban initial true-onset glides and initial onset rhotics while permitting initial onset laterals. Mbabaram does allow word-initial glides, showing that this language also permits glides to be syllabified as nuclear onglides rather than as true onsets. However, Sestu completely bans word-initial glides as well as rhotics, which correlates with the fact that this dialect also bans rising diphthongs; thus, nuclear onglides are in general disallowed in this language.

The theory of positional augmentation constraints predicts that there should be languages in which even more of the $\left[{ }^{*} \mathrm{ONSET} / \mathrm{X}\right] / \sigma_{1}$ hierarchy is ranked above faithfulness constraints, meaning that even more classes of segments (e.g., glides, liquids, and nasals) should be banned from word-initial position in some language. Recall that this prediction is borne out for the stressed-syllable version of this constraint subhierarchy (\$3.2.2.4), since Pirahã has even the comparatively lowranking [ ${ }^{*}$ ONSET/D]/ó ranked high enough to be active. Languages that show a tendency to avoid even medium-sonority onsets such as nasals or voiced segments, whether in stressed syllables, in initial syllables, or in all positions, are admittedly rare, but this may be simply because a language that restricts possible onsets in output forms to such a degree is less effective as a tool for communication and 
would therefore be subject to strong diachronic pressure to change its constraint ranking. ${ }^{28}$ Notably, there are languages that enforce many of the constraints of the *ONSET/X subhierarchy in reduplication, by violating faithfulness constraints on the $\mathrm{B}$ (ase)-R(eduplicant) correspondence relation (rather than on the I(nput)-O(utput) relation). Examples include Sanskrit, where it is the least sonorous member of a base onset cluster that is copied in the reduplicant (Steriade 1982, 1988), and Halq'eméylem', where sonorant onsets are avoided in reduplicants (Urbanczyk 1999a).

Further discussion of predicted and attested initial-syllable augmentation constraints is given in $\S 4.2 .3$ below.

\subsubsection{Positional augmentation in roots}

This section presents examples of positional augmentation effects in the other psycholinguistically strong position, the root. The root qualifies as a psycholinguistically strong position because roots are important in the organization of the lexicon and thus in how lexical entries are accessed (see $\$ 4.3 .3$ below). As a strong position, the root is predicted to resist positional neutralization, and such cases are indeed attested (see, e.g., McCarthy \& Prince 1995; Casali 1996; Beckman 1998; Alderete 1999b, 2001). Likewise, roots are predicted to undergo positional augmentation effects. Augmentation constraints that are seen to affect roots include HAVESTRESS/Root (\$4.2.2.1); it may also be the case that the familiar root-minimality effects are the result of root-specific augmentation constraints $(\S 4.2 .2 .2)$.

\subsubsection{Obligatory root stress: HAVESTRESS/Root}

One well-attested positional augmentation constraint for roots is HAVESTRESS/Root, formulated as follows (see $\$ 2.3 .2 .6$ on general HAVESTRESS).

28. One language that appears to have the ranking [*ONSET/D]/ $\sigma_{1} \gg$ IDENT[voice] is Bakairi (Wetzels \& Mascaró 2001). This language has several complicated restrictions on the voicing of obstruents, but the relevant fact for present purposes is that voiced and voiceless obstruents are contrastive, but obstruents in word-initial position can only be voiceless. Thanks to Rachel Walker for bringing this example to my attention. 
For all syllables $x$, if the head of $x$ is affiliated with a root, then $x$ bears stress

If this constraint is ranked high enough to be active, then a language will show a requirement for root stress. In some cases, as in Diegueño (Yuman; Langdon 1975, 1977), root stress is mandatory, indicating that HAVESTRESS/Root is undominated. There are also languages, such as Tuyuca (Tucanoan; Barnes 1996; Smith 1998), in which the requirement for root stress is subordinated, e.g., to the faithful preservation of input affix stress, but when all else is equal, root stress is enforced-an instance of "the emergence of the unmarked" (McCarthy \& Prince 1994). Additional languages that have been claimed to have a requirement for root stress include Tahltan (Athapaskan; Cook 1972, Nater 1989, Alderete 1999b, 2001), Chukchee (Paleo-Siberian; Krause 1979), Nancowry (Nicobarese; Radhakrishnan 1981), and Mbabaram (Australian; Dixon 1991).

It is important to note that not all languages that prefer to place stress on roots can serve as evidence for the M/Root constraint HAVESTRESS/Root. For example, Alderete (1999b, 2001) argues that the preference for root stress in Cupeño (UtoAztecan; Hill \& Hill 1968) has its basis in root-specific faithfulness (F/Root) constraints; an underlying root stress is preferentially realized over an underlying affix stress, but an underlying affix stress will be realized when the root is unstressed, just as in Tuyuca (see below). Crucially, however, when no morphemes in a word have an underlying stress, the surface form has stress on the word-initial syllable, whether or not it is part of the root. The default, markedness-driven pattern is thus left-edge stress, not root stress. This motivates the following ranking for Cupeño, in which HAVESTRESS/Root plays no role.

$$
\text { FAITH(Stress)/Root } \gg \text { FAITH(Stress) } \gg \text { ALIGN-L(ó, PrWd) }
$$

Thus, crucial evidence for HAVESTRESS/Root comes not from Cupeño, but from languages like Diegueño and Tuyuca, in which roots are compelled to have stress, rather than merely being given priority to realize an underlying stress when they have one.

Diegueño (Yuman; Langdon 1975, 1977) is a language in which roots always bear stress in output forms. (Here and in the following discussion, roots are underlined.)

$$
\text { Root stress in Diegueño (Langdon 1977:239-240) }
$$

(a) mát 
(b) tə-xə-mə- $\underline{k^{W} \text { án- }}-p$

(c) m-axku:xáp ${ }^{29}-c-m ə-y u$ you-catch.up-SAME.SUBJ-you-be 'is tangled up'

'Are you catching up with him?'

In a Diegueño-type language, with mandatory root stress, HAVESTRESS/Root must be ranked above all relevant faithfulness constraints, as well as above any markedness constraints (such as stress-alignment constraints or $\mathbf{M} /{ }^{\prime} \mathbf{o}$ constraints) whose satisfaction would cause stress to fall outside the root. A representative set of constraints that must be crucially dominated by HAVESTRESS/Root in a Diegueño-type language is shown in (60).

A language with mandatory root stress (hypothetical form)

\begin{tabular}{|r||c|c|c|c|}
\hline /ma+du+tá/ & HAVESTR/Rt & $\begin{array}{c}\text { ALIGN- } \\
\text { L(ó) }\end{array}$ & $\begin{array}{c}{[* \text { PEAK/V]/ó }} \\
\text { HIV }\end{array}$ & $\begin{array}{c}\text { FAITH } \\
\text { (Stress) }\end{array}$ \\
\hline \hline a. madúta & & $*$ & $*$ & $*$ \\
\hline b. mádutta & $* !$ & & & $*$ \\
\hline c. madutá & $* !$ & $* *$ & & \\
\hline
\end{tabular}

In Diegueño-type languages, the positional augmentation constraint HAVESTRESS/Root is undominated, so stress always appears on a root vowel. Unlike the ranking for Cupeño, the high-ranking constraint that compels root stress here cannot possibly be a positional faithfulness constraint such as MAX-PROM/Root (which penalizes the deletion of a root stress; Alderete 1999b, 2001), because the satisfaction of a faithfulness constraint, by definition, cannot force roots to have stress. The relevant constraint must be one that specifically requires the root to bear stress, that is, the M/Root constraint HAVESTRESS/Root.

Another language for which the existence of HAVESTRESS/Root is crucial is Tuyuca (Tucanoan; Barnes 1996; Smith 1998). Here, HAVESTRESS/Root is not undominated as it is in Diegueño-type languages. Tuyuca has a lexical contrast between stressed and unstressed roots, and between stressed and unstressed affixes.

29. Langdon (1977:239) notes that the stem a:kuxáp contains three prefixes, but she does not segment them. According to Langdon's $(1975,1977)$ descriptions, roots in Diegueño are always monosyllabic, so the root here must be áp or xáp. 
When a word is formed from a stressless root and a stressed affix, the surface form will have stress on the affix, violating HAVESTRESS/Root in order to satisfy faithfulness constraints. However, in situations where faithfulness to underlying stress is not at stake, the effects of HAVESTRESS/Root emerge. Namely, when a word in Tuyuca contains only stressless morphemes, the obligatory default stress is inserted into the root rather than into an affix, so that HAVESTRESS/Root is satisfied. Once again, a system that has no markedness constraint specifically requiring root stress is unable to account for this pattern.

According to Barnes (1996), stress in Tuyuca is assigned at the level of the PrWd, which consists of a root optionally followed by one or more suffixes. ${ }^{30}$ Both roots and suffixes can be underlyingly specified for stress, and the location of stress within roots is also lexically contrastive ${ }^{31}$ (suffix stress location is generally predictable; see Barnes 1996 for discussion).

(61) Lexical contrasts for stress in Tuyuca (data from Barnes 1996)

\begin{tabular}{|c|c|c|c|c|}
\hline \multirow[b]{2}{*}{ Stressed } & \multicolumn{2}{|l|}{ Roots } & \multicolumn{2}{|l|}{ Suffixes } \\
\hline & $\begin{array}{l}\text { hóa } \\
\text { póa } \\
\text { waí } \\
\text { kapéa } \\
\text { keeró } \\
\text { hóo } \\
\text { hoó }\end{array}$ & $\begin{array}{r}\text { 'to write' } \\
\text { 'hair' } \\
\text { 'fish' } \\
\text { 'eye' } \\
\text { 'lightning bug' } \\
\text { 'to plant manioc' } \\
\text { 'to cut slashes' }\end{array}$ & $\begin{array}{l}\text {-mênã } \\
\text {-mãkê } \\
\text {-díki } \\
- \text { sotoá } \\
\text {-jú } \\
- \text {-wí } \\
\text {-gó }\end{array}$ & $\begin{array}{r}\text { 'with' } \\
\text { 'stuff } \\
\text { 'only' } \\
\text { 'on top of } \\
\text { 'beforehand' } \\
\text { (an evidential) } \\
\text { (fem. sg. vb. sfx.) }\end{array}$ \\
\hline 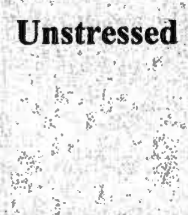 & $\begin{array}{l}\text { hoo'to } \\
\text { nõã } \\
\text { waka } \\
\text { waso }\end{array}$ & $\begin{array}{r}\text { submerge oneself } \\
\text { 'who' } \\
\text { 'splinter' } \\
\text { 'to change' }\end{array}$ & $\begin{array}{l}-a \\
-i \\
-j e \\
-s a \text { (ther }\end{array}$ & $\begin{array}{r}\text { (an evidential) } \\
\text { (an evidential) } \\
\text { (change of focus) } \\
\text { ematic importance) }\end{array}$ \\
\hline
\end{tabular}

30. There is a two-morpheme window for stress assignment in Tuyuca-all morphemes after the first two in a PrWd (or the first three, in certain circumstances involving classifiers) are ignored in determining the placement of stress. See Barnes (1996:46 ff.) for discussion. Since Tuyuca has no prefixes, the root will always be included in the stress-assignment window.

31. The verb roots analyzed by Barnes (1996) as having a lexically specified floating stress are reanalyzed in Smith (1998) with lexical stress on the root-final mora. 
A stressed root is one that bears stress regardless of what suffix is attached to it (62a), while an unstressed root is one that bears stress when concatenated with certain suffixes but not with others (62b). A stressed suffix is one that bears stress when attached to an unstressed root $^{32}(62 \mathrm{c})$; an unstressed suffix is one that never bears stress $(62 d)$.

Stressed and unstressed morphemes in combination (Barnes 1996:41)

\begin{tabular}{|c|c|c|c|}
\hline & \multicolumn{2}{|l|}{ Roots } \\
\hline & & stressed & unstressed \\
\hline \multicolumn{2}{|c|}{ Suffixes } & (a) /hóa/ & (b) /waso/ \\
\hline stressed & (c) /-jú/ (ASP.) & hóaju & wasojú \\
\hline unstressed & (d) $/-\mathrm{i} /$ (EV.) & hóai & wasói \\
\hline
\end{tabular}

Thus, surface stress appears on an affix when a lexically stressed affix is combined with a lexically unstressed root. This fact indicates that MAX-PrOM (63), which bans the deletion of input stress specifications, must dominate HAVESTRESS/Root, as shown in (64).

MAX-PROM

A metrical prominence (=stress) in the input must have an output correspondent. (Alderete 1999b, 2001)

32. Affix stress also surfaces when a verb with final stress is combined with a monosyllabic nominalizer. In this case, the verb stress is deleted, and the affix stress is realized. The analysis presented in Smith (1998) accounts for this pattern by means of a noun-specific positional faithfulness constraint that prevents deletion of stress in morphemes that are $[+\mathrm{N}($ oun $)]$, including nominalizers. 
(64) Stressed affix and unstressed root: affix bears stress

/hoo + wi/ submerge.oneself-EV 'he submerges himself'
\begin{tabular}{|r||c|c|}
\hline /hoo+wi// & MAX-PROM & HAVESTRESS/Rt \\
\hline \hline a. hoówi & $* !$ & \\
\hline b. hoowi & & $*$ \\
\hline
\end{tabular}

Because the faithfulness constraint MAX-PROM outranks HAVESTRESS/Root, the latter can never force stress to fall on a lexically unstressed root rather than on a lexically stressed affix. However, the effects of HAVESTRESS/Root emerge whenever MAX-PROM is inactive. One such situation arises when a PrWd is composed entirely of unstressed morphemes, as in (65). Since there are no input stresses to be deleted, no candidate violates MAX-PROM. The undominated constraint CULMINATIVITY requiring every PrWd to have exactly one stress (see \$2.3.2.6) forces stress insertion, but the choice between root stress (65b) and affix stress $(65 \mathrm{c})$ falls to HAVESTRESS/Root, which selects the candidate with root stress. $^{33}$

No underlying stress: default stress falls on root

/hoo + a/ submerge.oneself-EV 'I submerge myself

\begin{tabular}{|r||c|c|c|}
\hline /hoo + a/ & CULMinAtivity & $\begin{array}{c}\text { MAX- } \\
\text { Prom }\end{array}$ & HAVESTRESS/Rt \\
\hline \hline a. hooa & $* !$ & & $*$ \\
\hline b. hoóa & & & \\
\hline c. hooá & & & $* !$ \\
\hline
\end{tabular}

Since there are both general and root-specific faithfulness constraints (McCarthy \& Prince 1995; Beckman 1998; Alderete 1999b, 2001), insertion of a

33. Barnes (1996) states that default stress is always inserted on the rightmost vowel (mora) of the root, which indicates that the ranking HAVESTRESS/Root $>$ ALIGN-R(ó, PrWd) determines default stress placement. Crucially, it is not simply left-edge alignment of stress that is responsible for placing stress on the root rather than on a suffix. 
default stress into a root is a greater faithfulness violation than insertion of the stress into the affix (66).

(66) Faithfulness violations for stress insertion

\begin{tabular}{|c||c|c|}
\hline /hoo + a/ & DEP-PROM/Root & DEP-PROM \\
\hline \hline a. hoóa & $* !$ & $*$ \\
\hline b. hooá & & $*$ \\
\hline
\end{tabular}

No matter how DEP-PROM and DEP-PROM/Root are ranked with respect to one another, insertion of default stress into a root rather than into an affix could never be optimal if these were the only relevant constraints in the system. Default stress on roots can be compelled only if HAVESTRESS/Root is part of the system and outranks DEP-PROM/Root (67). (The ranking of general DEP-PROM with respect to HAVESTRESS/Root is irrelevant, since undominated CULMINATIVITY will force one violation of general DEP-PROM whenever there is no input stress.)

HAVESTRESS/Root compels root-stress insertion

\begin{tabular}{|c|c|c|c|}
\hline /hoo + a/ & HAVESTRESS/Rt & DEP-PROM/Rt & DEP-PROM \\
\hline \hline a. hoóa & & $*$ & $*$ \\
\hline b. hooá & $* !$ & & $*$ \\
\hline
\end{tabular}

The other configuration in which HAVESTRESS/Root shows emergent effects is a word in which there is a lexically specified stress both on the root and on an affix, as in (68). In this case, undominated CULMINATIVITY rules out any candidate with more than one stress. All candidates that satisfy CULMINATIVITY violate MAXPROM equally, so MAX-PROM is not active in determining the winner. Again, the decision falls to HAVESTRESS/Root, which chooses root stress (68b) over affix stress $(68 \mathrm{c})$. 
Multiple underlying stresses: root stress survives

/hóo + wi/ plant.manioc-EV 'he plants manioc'

\begin{tabular}{|r||c|c|c|}
\hline /hóo+wi/ & CULMINATIVITY & MAX-Prom & HAVESTR/Rt \\
\hline \hline a. hóowi & $* !$ & & \\
\hline b. hóowi & & $*$ & \\
\hline c. hoowi & & $*$ & $* !$ \\
\hline
\end{tabular}

A candidate that deletes an underlying affix stress would also be chosen over one that deletes an underlying root stress, as in (68), by faithfulness constraints, just as in Cupeño (Alderete 1999b, 2001)-deleting an affix stress violates only MAXProm, but deleting a root stress violates both MAX-PrOM and MAX-PrOM/Root. However, there is no faithfulness alternative for the obligatory insertion of stress into roots (as in (67)). For the insertion case, HAVESTRESS/Root is crucial. Thus, Tuyuca provides additional evidence for the inclusion of HAVESTRESS/Root in the universal constraint inventory.

The relevant constraint ranking for Tuyuca is summarized in (69).

$$
\begin{aligned}
& \text { CULMINATIVITY }>>\text { MAX-PROM }>>\text { HAVESTRESS/Root }>>\text { DEP-PROM/Root } \\
& \longrightarrow \text { DEP-PROM }
\end{aligned}
$$

Undominated CULMINATIVITY ensures that every PrWd has one and only one stress. MAX-PROM >> HAVESTRESS/Root means that default root stress will never be assigned if there is an underlyingly stressed affix available. But even though HAVESTRESS/Root is dominated, its effects emerge in cases where MAX-PROM is inactive; the crucial case is a word in which no morpheme has an underlying stress, so that a default stress must be inserted.

In summary, HAVESTRESS/Root is an augmentation constraint that acts to enhance the strong position root with the prominent property stress. When this constraint outranks stress-location constraints and faithfulness constraints that preserve underlying affix stress, then stress always falls on roots. Even when this constraint is dominated by faithfulness constraints so that some words bear stress on their affixes, its effects may emerge as a requirement that inserted default stresses fall on roots. 


\subsubsection{Root minimality effects}

In addition to the root-stress requirement considered in $\$ 4.2 .2 .1$, there is another property that is often enforced specifically of roots: in many languages, roots are subject to minimal-word (i.e., bimoraic or disyllabic) size requirements. Such requirements result from demands that roots be coextensive with prosodic words (Prince 1980; Broselow 1982; Crowhurst 1992; McCarthy \& Prince 1986, 1990). Minimality requirements on PrWds are themselves obtained from the prosodic hierarchy: a PrWd must contain a foot, and (non-degenerate) feet are minimally binary.

The question that must be addressed, then, is how to ensure that a root contains a whole PrWd. Minimality effects have most often been analyzed within OT, following McCarthy \& Prince (1993ab), as the combined effect of separate alignment constraints demanding that the left and right edges of the morphological category in question to be aligned with the analogous edges of PrWds. Aligning the left edge of a root with the left edge of a PrWd, and the right edge of a root with the right edge of a PrWd, entails that the root contains (at least) one PrWd.

This approach to root minimality requirements, which can be called the "double-edge alignment" approach, is quite attractive; it forces roots to match up with PrWds using nothing but alignment constraints, the existence of which is independently motivated. However, the requirement that roots fill out a prosodic constituent of a particular size is reminiscent of an augmentation process-ensuring that roots are fairly large does have the effect of ensuring that they are perceptually prominent. But formally, there is no way to view the double-edge alignment approach to root minimality as a kind of root augmentation, since roots are mapped onto PrWds only when two independent alignment constraints (for the two edges, $\mathrm{L}$ and $\mathrm{R}$ ) happen both to be high ranking. Under this approach, there is no single constraint that requires roots to contain PrWds or otherwise forces them to be large.

Several previous approaches to root minimality effects did have more of the flavor of root augmentation constraints, however. For example, Prince \& Smolensky's (1993:43) constraint $\mathrm{LX} \approx \operatorname{Pr}(M C a t)$ 'A member of the morphological category $M C a t$ correspond[s] to a PrWd' was designed to be relativized to different morphological categories, so that, e.g., roots, stems, and MWds could each be compelled to equal a PrWd in size. Similarly, earlier work by McCarthy \& Prince (1991 [cited in McCarthy \& Prince 1993a]) makes use of morphology/prosody output constraints of the form 'MCat=PCat,' a schema which would allow for the instantiation 'Root=PrWd.'

Furthermore, it has been argued in Smith $(1998,1999,2001)$ that the lexical category noun behaves as a strong position with respect to avoidance of positional neutralization. As yet, no unambiguous instances of noun augmentation have come 
to light, but there is at least one language with noun-specific minimality effects: in Chuukese [Trukese] (Muller 1999), nouns must be bimoraic, but verbs need not be; furthermore, monomoraic verbs, without affixation, are well-formed utterances.

Thus, roots and nouns, both of which are strong positions, are both seen to have minimality requirements. This is at least suggestive that the existence of prosodic minimality requirements for a lexical/morphological constituent is related to its status as a strong position. However, additional investigation is needed to determine whether it is appropriate to treat minimal-size requirements as a type of augmentation effect (with a single size-enforcing constraint that can be relativized to strong positions like roots and nouns, perhaps formalized as a word-level counterpart to Truckenbrodt's (1999) phrasal WRAP constraints), or whether the formally simpler double-edge alignment approach is preferable, despite its lack of any fundamental connection to the status of an element as a strong position.

\subsubsection{Summary: Root augmentation}

Roots, as strong positions, are predicted to be eligible for positional augmentation (M/Root) constraints. This prediction is borne out: the constraint HAVESTRESS/Root is active in a number of languages, overriding faithfulness (and root faithfulness) constraints and requiring output forms to place stress within the root. Root minimality effects are another possible case of positional augmentation in roots, but further research is required before specific claims can be made about the constraints responsible for minimality effects.

\subsubsection{Conclusion: Predicted and attested $M / \Psi$ str constraints}

This section has presented examples of languages in which positional augmentation constraints for psycholinguistically strong positions are active: ONSET $/ \sigma_{1}$, $\left[{ }^{*} \mathrm{ONSET} / \mathrm{X}\right] / \sigma_{1}$, and HAVESTRESS/Root. As discussed in $\$ 2.4$, fewer positional augmentation constraints are predicted to exist for psycholinguistically strong positions than for phonetically strong positions; the former are important in earlystage word recognition, so there are substantive reasons to avoid neutralizing crucial contrasts - that is, contrasts in features that are relevant in the early stages of speech perception-in these positions.

The constraint filter that formally represents this substantive pressure is the Segmental Contrast Condition (introduced in $\$ 2.4 .1$, with further discussion and justification in $\S 4.3$ below). This filter screens out $\mathbf{M} / \Psi$ str constraints whose satisfaction would require the neutralization of contrasts that are relevant in early- 
stage word recognition. Since contrasts involving stress are not important at that stage of processing (see $\S 4.3 .4 .1$ for discussion), HAVESTRESS/Root is a legitimate

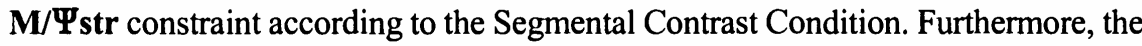
Segmental Contrast Condition has a disjunctive formulation, such that an $\mathbf{M} / \Psi$ str constraint is also acceptable if the strong position is the initial syllable and the constraint is one that enforces low sonority at the left edge. ONSET $/ \sigma_{1}$ and the members of the $\left[{ }^{*} \mathrm{ONSET} / \mathrm{X}\right] / \sigma_{1}$ subhierarchy are therefore able to pass the Segmental Contrast Condition as well.

However, a number of other formally possible $\mathbf{M} / \Psi$ str constraints (which, moreover, pass the Prominence Condition) are predicted not to pass the Segmental Contrast Condition. These are indicated in (70) with the notation 'SCC.'

(70) Predicted positional augmentation constraints for psycholinguistically strong positions

\begin{tabular}{|c|c|c|}
\hline & $\sigma_{1}$ & root \\
\hline ONSET/str & $\begin{array}{l}\text { - Arapaho } \\
\text { - Guhang Ifugao }\end{array}$ & $S C C$ \\
\hline$\left[{ }^{*} \mathrm{ONSET} / \mathrm{X}\right] / s t r$ & $\begin{array}{l}\text { - Campidanian Sardinian } \\
\text { - Mongolian, Kuman, etc. } \\
\text { - Mbabaram }\end{array}$ & $S C C$ \\
\hline$\left[{ }^{*} \mathrm{PEAK} / \mathrm{X}\right] / s t r$ & SCC & $S C C$ \\
\hline HAVECPLACE/str & $\begin{array}{l}\text { domain } \\
\text { mismatch }\end{array}$ & $S C C$ \\
\hline HEAVYo/str & $S C C$ & $S C C$ \\
\hline HAVESTRESS/str & $? ?$ & $\begin{array}{l}\text { - Diegueño } \\
\text { - Tuyuca }\end{array}$ \\
\hline HTONE/str & ?? (SCC?) & ?? (SCC?) \\
\hline
\end{tabular}

For initial syllables, any constraint that refers to segmental contrasts, but whose satisfaction does not aid in left-edge demarcation, will fail to pass the Segmental Contrast Condition. Such constraints include $\left[{ }^{*} \mathrm{PEAK} / \mathrm{X}\right] / \sigma_{1}$ and HEAVYo/ $\sigma_{1}$. A further constraint that is predicted not to exist is HAVECPLACE $/ \sigma_{1}$; relativizing HAVECPLACE to the position initial syllable leads to a domain mismatch $(\S 2.2$, 
$\S 2.3 .3$ ), since the focus of this constraint makes reference to consonants, not syllables.

As discussed in $\S 2.3 .3$, any of the markedness constraints in (70) can be relativized to the root with no domain mismatch, since the root is defined with reference only to morphological affiliation, not to segments or to prosodic categories. Nevertheless, most of these constraints are prohibited from having M/Root counterparts because of the Segmental Contrast Condition. For roots, there is no left-edge escape clause, so any constraint whatsoever that refers to a segmental contrast will be ruled out by the Segmental Contrast Condition; these include ONSET/Root, [*ONSET/X]/Root, [ ${ }^{*}$ PEAK/X]/Root, HAVECPlaCE/Root, and HEAVYo/Root.

The three cells remaining in the chart in (70), representing positional augmentation constraints for which no examples have been presented here, but which are not (unambiguously) predicted to be ruled out by the Segmental Contrast Condition or ill-formed because of a domain mismatch, are indicated with '??.' The constraints in question are HAVESTRESS $/ \sigma_{1}$, HTONE/ $\sigma_{1}$, and HTONE/Root. The remainder of this section considers each of these three potential $\mathbf{M} / \Psi$ str constraints.

The positional augmentation constraint HAVESTRESS $/ \sigma_{1}$ is very clearly predicted to exist. The simple fact that HAVESTRESS/Root exists confirms that HAVESTRESS/ $\Psi$ str constraints pass the Segmental Contrast Condition; furthermore, experimental results show that stress does not play the same role as segmental contrasts in early-stage word recognition (see §4.3.4).

While there is at this time no conclusive evidence in support of the existence of HAVESTRESS $/ \sigma_{1}$, there is also no evidence that it does not exist. It is uncontroversial that some languages have stress on initial syllables; in fact, as demonstrated by the cross-linguistic survey of stress patterns in Hyman (1977), initial-stress languages are common. Languages with mandatory initial stress include, for example, a large number of the Australian languages, Czech, Cahuilla, and many of the Finno-Ugric languages. If there is a positional augmentation constraint HAVESTRESS $/ \sigma_{1}$, then it can account for languages with mandatory initial stress. 
(71) Initial stress by HAVESTRESS $/ \sigma_{1}$

\begin{tabular}{|c|c|c|}
\hline /tatáta/ & HAVESTRESS/ $\sigma_{1}$ & FAITH(Stress) \\
\hline \hline a. tátata & & $*$ \\
\hline b. tatáta & $* !$ & \\
\hline c. tatatá & $* !$ & $*$ \\
\hline
\end{tabular}

However, languages with mandatory initial stress can also be accounted for with a high-ranking ALIGN-L( $\dot{\sigma}, \mathrm{Wd})^{34}$ constraint, as demonstrated for example in Walker (1996).

Initial stress by ALIGN-L(ó, Wd)

\begin{tabular}{|r||c|c|c|}
\hline /tatáta/ & $\begin{array}{c}\text { ALIGN-L } \\
(\mathfrak{\sigma}, \mathrm{Wd})\end{array}$ & $\begin{array}{c}\text { ALIGN-R } \\
(\mathfrak{\sigma}, \mathrm{Wd})\end{array}$ & FAITH(Stress) \\
\hline \hline a. tátata & & $* *$ & $*$ \\
\hline b. tatáta & $* !$ & $*$ & \\
\hline c. tatatá & $* ! *$ & & \\
\hline
\end{tabular}

Where initial stress is mandatory, such that no form in the language ever violates the constraint calling for it, then there is no way to determine whether initial stress is caused by HAVESTRESS $/ \sigma_{1}$ or by ALIGN-L $(\dot{\sigma}, W d)$. However, as seen in (71) and (72), the two constraints have different patterns of violability. ALIGN-L(ó, Wd), being an alignment constraint (McCarthy \& Prince 1993a), is gradiently violable, but HAVESTRESS $/ \sigma_{1}$ is categorically evaluated (either the initial syllable has stress, or it does not).

34. The ALIGN-L constraint that most closely overlaps with HAVESTRESS $/ \sigma_{1}$ is ALIGN-L(ó, MWd), because (as argued in $\S 4.4$ below) the strong position "initial syllable" is best characterized as the MWd-initial syllable. However, in many stress systems, it will be difficult to distinguish empirically between ALIGN-L(ó, MWd) and ALIGN-L(ó, PrWd). In other words, ALIGN-L(ó, MWd) and ALIGN-L(ó, PrWd) are both alignment constraints that are potentially responsible for enforcing left-edge stress. 
Because ALIGN-L(ó, Wd) is gradient, it may still have partial effects in languages where it is crucially dominated -if stress cannot be leftmost, because that would violate some higher-ranked constraint, stress will at least be as far to the left as possible. This is the pattern seen in Western Arrernte stress (\$3.2.2.2): stress is initial except in vowel-initial words, where it appears on the second syllable to satisfy ONSET/ó (subject to NONFINALITY).

(73) Western Arrernte main stress (Downing 1998; data from Strehlow 1942)

(a) rá:tama 'to emerge'
kútungula 'ceremonial assistant'

(b) arálkama 'to yawn'

ulúrba 'cold; cold wind'

As seen in $\S 3.2$, this kind of nearly-edge-bound stress pattern comes about when a higher-ranking constraint (in this case, ONSET/ó) dominates ALIGN-L(ó, Wd). Crucially, the alignment constraint is only minimally violated; stress still falls as far to the left as possible, while appearing on a syllable with an onset.

$$
\text { Gradient violation of ALIGN-L(ó, Wd) in Arrernte }
$$

\begin{tabular}{|r||c|c|}
\hline /aralkama/ & ONSET/ó & ALIGN-L(ó, Wd) \\
\hline \hline a. áralkama & $* !$ & \\
\hline b. arálkama & & $*$ \\
\hline c. aralkamá & & $* * * !$ \\
\hline
\end{tabular}

The question now arises as to whether there are any stress systems with a tendency toward left-edge stress, but where violation of the left-stress constraint is categorical rather than gradient. If there are languages where stress, if prevented from falling on the initial syllable, shows no left-edge tropism but falls somewhere else entirely, then this would provide stronger evidence for the existence of HAVESTRESS $/ \sigma_{1}$ in addition to Align-L(o, Wd).

The status of HTONE $/ \sigma_{1}$ and HTONE/Root is less clear. Whether or not these are predicted to be legitimate $\mathbf{M} / \Psi$ str constraints depends on the degree to which tonal contrasts are utilized in early-stage word recognition, a question that is far from settled (see $\S 4.3 .4 .2$ below). If these constraints do exist, there should be 
languages in which initial syllables and roots obligatorily bear a $\mathrm{H}$ tone, so that (for example) $\mathrm{H}$ tones underlyingly associated with other syllables in the word either move or spread to the initial syllable or root respectively.

In summary, $\S 4.2$ has presented evidence for the existence of certain $\mathbf{M} / \Psi$ str constraints, namely, ONSET $/ \sigma_{1},\left[{ }^{*} \mathrm{ONSET} / \mathrm{X}\right] / \sigma_{1}$, and HAVESTRESS/Root, and has discussed certain theoretical implications of the existence of these constraints (especially the implications of [ $\left.{ }^{*} \mathrm{ONSET} / \mathrm{X}\right] / \sigma_{1}$ for the syllabification of pre-peak glides). The discussion here has also shown that the comparatively restricted inventory of $\mathbf{M} / \Psi$ str constraints is predicted by the constraint filter known as the Segmental Contrast Condition (§2.4). The next section, §4.3, examines in more detail the psycholinguistic basis of the Segmental Contrast Condition.

\subsection{Psycholinguistic evidence behind the Segmental Contrast Condition}

This section presents psycholinguistic evidence in support of the substantive factors that are formalized in the Segmental Contrast Condition, the filter on $M / \Psi$ str constraints introduced in $\$ 2.4 .1$, which is repeated here in (75).

\section{Segmental Contrast Condition}

If a constraint is of the form $\mathbf{M} / \Psi$ str, then it must meet one of the following two conditions:

I. Satisfaction of the $\mathbf{M}$ constraint from which the $\mathbf{M} / \Psi$ str constraint is built does not alter features that are distinguished in early-stage word recognition.

or

II. $\Psi$ str is $\sigma_{1}$, and satisfaction of the $M / \Psi$ str constraint serves to demarcate the left edge of $\sigma_{1}$.

First, $\$ 4.3 .1$ presents an overview of the process of word recognition, which serves as background for the discussion of relevant psycholinguistic findings in the remainder of $\S 4.3$. 4 4.3.2 then shows that word-initial material is of particular importance in determining the set of lexical entries to be considered in early-stage word recognition, and $\S 4.3 .3$ shows that roots have a special role in how the lexicon is organized and hence in how words are accessed. These two conclusions support, 
first, the identification of initial syllable and root as psycholinguistically strong positions; second, the definition of psycholinguistically strong positions as those positions that are of special importance in early-stage word recognition (as distinct from the stressed syllable, which is discussed in §4.3.4); and third, the idea that there are substantive reasons to avoid neutralizing phonological contrasts in these positions (a point further discussed in $\S 4.3 .5$ ).

$\S 4.3 .4$ then presents evidence that segmental and prosodic properties have fundamentally different roles in word recognition, motivating the narrow domain of relevance of the Segmental Contrast Condition: namely, its inapplicability to the strong position stressed syllable and its attention to segmental contrasts specifically. First, results are reviewed showing that stressed syllables are not used directly to access lexical items (although they are used in speech perception in other ways, such as in the related but distinct task of word segmentation). This is why the strong position stressed syllable is not subject to the Segmental Contrast Condition, a proposal that is empirically supported by the wide variety of $\mathbf{M} / \mathbf{\sigma}^{\mathbf{\sigma}}$ constraints discussed in Chapter 3-while not irrelevant for speech perception, the stressed syllable is not a psycholinguistically strong position in the strict sense. This subsection also examines psycholinguistic evidence bearing on the question of whether tonal contrasts play the same kind of role in early-stage word recognition that segmental contrasts do. In short, the discussion shows that there is no substantive reason to avoid $\mathbf{M} / \Psi$ str constraints such as HAVESTRESS/Root and HAVESTRESS $/ \sigma_{1}$ (and, perhaps, HTONE/Root and HTONE/ $\sigma_{1}$ ); neutralizations affecting the prosodic properties relevant for such constraints have much less of an impact on the efficiency of word recognition. And as seen in $\S 4.2$ above, $\mathbf{M} / \Psi$ str constraints involving prosodic properties like stress are indeed attested.

Finally, $\S 4.3 .5$ concludes the discussion of the psycholinguistic evidence by showing how that evidence supports the particular formulation that has been proposed for the Segmental Contrast Condition. Namely, decreasing the number of segmental contrasts available in the crucial positions initial syllable and root would have an adverse effect on word recognition (clause I). However, the ability to demarcate word boundaries clearly (as through satisfaction of ONSET/ $\sigma_{1}$ and members of the [ ${ }^{*}$ ONSET $\left./ \mathrm{X}\right] / \sigma_{1}$ subhierarchy) is useful enough in speech perception to override the general avoidance of segmental-contrast neutralization in psycholinguistically strong positions (clause II).

\subsubsection{Word recognition}

Before the substantive pressures on psycholinguistically strong positions that are modeled in the Segmental Contrast Condition-and, more generally, the relevance 
of psycholinguistically strong positions for word recognition-can be considered in detail, some background discussion of the process of word recognition is necessary. (See also, e.g., Altmann 1990a, Forster 1990, Garrett 1990, and Handke 1995 for overviews of various aspects of word recognition and lexical access.)

Spoken-language understanding happens extremely quickly and at a level that is essentially inaccessible to conscious introspection. In the course of this process, a hearer receives acoustic input, matches that input to entries in his or her mental lexicon, and uses the syntactic and semantic information provided by the selected lexical entries (as well as non-linguistic factors such as the discourse context) to arrive at an interpretation of the utterance. Word recognition, one component of this procedure, is the process by which an incoming acoustic signal is matched with lexical entries so that the words in the utterance can be identified. ${ }^{35}$

The results of a body of psycholinguistic research indicate that there are two stages in word recognition. When an acoustic signal is first perceived, a set of lexical entries that resemble the incoming acoustic signal is activated, with no influence from syntactic or semantic context. Then, this initially activated set of lexical entries is quickly pared down until the single entry that is the best fit, phonologically and also syntactically and semantically, is identified.

Evidence for a distinction between early and later stages of word recognition comes, for example, from cross-modal priming experiments. Cross-modal priming is an experimental paradigm based on the finding that semantic priming (exposure to a semantically related word) causes a faster lexical-decision response ("word or nonword?") to a target word presented shortly after the prime. In a cross-modal priming experiment, participants listen to an auditory stimulus and are asked to make a lexical decision about a visually presented target. If some element in the auditory input causes the activation of a particular lexical entry, then the response time to a lexical decision for a visually presented target that is semantically related to the activated lexical entry should be faster than in control cases (where the visual target is unrelated to any lexical entry that would be activated by the auditory stimulus).

Cross-modal priming studies have shown that when a lexically ambiguous word such as bug or rose is first perceived, both of the corresponding lexical entries are initially activated, but within about two hundred milliseconds (equivalent to two or

35. Some sources use the term lexical access for this process. Both word recognition and lexical access are used fairly inconsistently in the literature (see Handke 1995: $\$ 1.3$ for discussion); here, the term word recognition is used in the sense defined above, and lexical access is used more broadly to refer to aspects of speech processing that involve searching the lexicon or making use of lexically stored information. 
three syllables), only the appropriate entry remains activated (Swinney 1979; Seidenberg, Tanenhaus, Leiman, \& Bienkowski 1982). For example, Seidenberg et al. found that, for the auditory stimulus They all rose, priming effects for the visual target FLOWER are observed when presented at the offset of rose, indicating that the homophonous lexical entries $\operatorname{rose}_{\mathrm{V}}$ and $\operatorname{rose}_{\mathrm{N}}$ are both activated at this point (even though only the verb is a syntactically appropriate choice). However, if the visual target FLOWER is presented after a $200 \mathrm{msec}$ delay, no priming effects are observed, indicating that the inappropriate rose $_{\mathrm{N}}$ entry is no longer activated.

It is not only homophonous lexical entries that are activated when an auditory stimulus is encountered. A number of studies (including some of those reviewed in \$4.3.2-4 below) show that, more generally, a set of phonologically similar lexical entries is initially activated when input is processed. For example, Zwitserlood (1989) found that Dutch listeners showed priming effects for both geld 'money' and boot 'boat' on the offset of the auditory stimulus [kapi:], which is the initial portion of both kapitaal 'capital' and kapitein 'captain.' Similarly, Marslen-Wilson \& Zwitserlood (1989) found a certain degree of priming for bij 'bee' on the offset of woning 'dwelling,' a word that has a large amount of phonological overlap with honing 'honey'; this shows that listeners who hear woning activate honing (and therefore semantically prime bij). (However, Marslen-Wilson and Zwitserlood argue that a lexical entry is most strongly activated when it shares initial material with the auditory input; see $\$ 4.3 .2 .2$ ).

Thus, initial contact with an auditory stimulus results in a certain subset of the lexicon being activated, and the initially activated subset of the lexicon is chosen only on the basis of phonetic/phonological information. Subsequent lexical processing examines the activated entries for goodness of phonological fit as well as for syntactic and semantic compatibility. ${ }^{36}$ It is the stage of initial activation that

36. Many of the auditory-processing models that have been proposed directly incorporate a two-stage model of word recognition. Examples include the Search model (Forster 1976, 1990), the Cohort model (Marslen-Wilson \& Welsh 1978; Marslen-Wilson 1984, 1987), the Shortlist model (Norris 1994; Norris, McQueen, \& Cutler 1995), and the Neighborhood Activation Model (Luce 1986; Luce \& Pisoni 1998). These models differ in the factors and mechanisms that they consider to be involved in selection and competition among lexical entries (see $\S 4.3 .5$ for additional discussion). However, they all propose that word recognition has two stages: a first stage, in which phonetic/phonological information is used to identify a set of candidate lexical entries for further examination, and a later stage, in which the selected set is narrowed down (often on the basis of more than just phonetic or phonological information) until the best-matching lexical entry is identified.

One influential model of speech perception that does not incorporate two distinct stages of word recognition is TRACE (McClelland \& Elman 1986). However, see Norris (1994) 
is referred to in the discussion that follows as early-stage word recognition; §§4.2-4 present more detailed evidence supporting the claim that the initial syllable and the root are particularly important in early-stage word recognition, that the stressed syllable is not directly involved in this stage of processing, and that segmental features are involved in choosing the subset of lexical entries for initial activation but prosodic properties such as stress are less relevant at this stage.

\subsubsection{The importance of word-initial material in early-stage word recognition}

There is a considerable amount of psycholinguistic evidence that word-initial material plays a particularly important role in early-stage word recognition. In general, listeners have been shown to pay more attention to initial material than to later material, and to be more adversely affected by the disruption or mispronunciation of initial material than of material later in the word (\$4.3.2.1). Word-initial material has also been more directly shown to have a strong influence on early-stage word recognition (\$4.3.2.2). It is considerations of the kind addressed in this subsection that justify the identification of the word-initial position as a psycholinguistically strong position with special phonological status.

\subsubsection{Word-initial material as a focus of perceptual attention}

Brown \& McNeill (1966) and Browman (1978) investigate the "tip-of-the-tongue" (TOT) phenomenon, in which experimental participants have partially, but not completely, recalled a particular word. Brown \& McNeill (1966), using a written questionnaire study, found that the characteristic of the word most likely to be recalled by participants in a TOT state was the initial segment, although final material was more likely to be recalled than medial material. ${ }^{37}$ Browman (1978) reports that providing a participant with the initial segment, or confirming the

on the advantages of a two-stage model such as Shortlist over a model that, like TRACE, attempts to search the entire lexicon at once without first identifying a subset of relevant entries to consider.

37. Interestingly, Brown \& McNeill (1966) show that words recalled by participants in a TOT state that are related by meaning, rather than by sound, to the as-yet unrecalled target word are more likely to be similar to the target at the right edge than initially, suggesting that this may reflect a general tendency for English words to resemble each other more finally than initially. They conclude that "the relative superiority of the SS [similar-sound] curve is greater in the first three positions [than in the final three]" (emphasis in original). 
participant's recollection of the initial segment, was the most successful way to bring the participant out of a TOT state and promote actual recall of the target word.

Cole (1973) and Cole \& Jakimik $(1976,1980)$ report the results of experiments in which participants were asked to detect deliberate mispronunciations in the auditory presentation of a short story. Cole (1973) found that listeners were more likely to detect a change in multiple features than a change in only one (i.e., voicing or place), but that a one-feature change was more likely to be detected in initial position than in the second or third syllable. Cole \& Jakimik $(1976,1980)$ found that, in disyllabic words, stress facilitates the detection of mispronunciations equivalently in initial and second syllables (see also $\$ 4.3 .4 .1 .1$ ), but they confirm Cole's (1973) findings when unstressed syllables are considered: a mispronunciation in an initial unstressed syllable is more likely to be detected than a mispronunciation in a non-initial unstressed syllable.

Marslen-Wilson (1975) and Marslen-Wilson \& Welsh (1978) present evidence from speech-shadowing tasks that mispronunciations are more likely to be perceived in initial, rather than later, portions of words. In speech shadowing, participants listen to auditory input and repeat it aloud. These studies found that listeners were much less likely to "restore" a mispronunciation (i.e., repeat it back as the correctly pronounced word, indicating that they have not noticed the mispronunciation) when it occurred in an initial syllable. Even when the mispronounced words were embedded in syntactic and semantic contexts that gave strong cues for what the correctly pronounced word should be, leading to a high rate of restoration in non-initial syllables, there were few instances of initial-syllable restorations. Marslen-Wilson (1984), reviewing these results, concludes that the effects of an initial mispronunciation on auditory processing are much greater than those of a mispronunciation in a later position.

Finally, Mattys \& Samuel (2000) found that response times for phoneme monitoring (a task in which participants are asked to respond when they hear a particular phoneme) were faster when the target phonemes were onsets to initial syllables than when they were in other positions.

Thus, the studies reviewed here all indicate that listeners are more aware of, or pay greater attention to, material in the initial portion of the word.

\subsubsection{A special role for initial material in early-stage word recognition}

Given that word-initial material is, chronologically, the first portion of a word that a hearer encounters, and that hearers apparently pay special attention to initial material, it has the potential to play an important role in word recognition 
(Nooteboom 1981; Marslen-Wilson 1984). Several studies provide evidence that this is in fact the case.

Nooteboom (1981) shows that initial fragments are more useful than final fragments in identifying words. He identified a set of (Dutch) words that can be divided in two parts such that both the initial part and the final part are unique. For example, the word surrogaat 'substitute' is the only word that begins [sœro:], and the only word that ends [o:' $\left.\chi a^{\prime} t\right]$. When these fragments were played for listeners to identify, accuracy was higher and response times were faster for the initial fragments than for the final fragments.

Cole (1973) and Cole \& Jakimik (1980) report that response times for the detection of mispronunciations, measured from the onset of the mispronounced phoneme, were longer when the mispronunciation occurred in the onset of the initial syllable than when it occurred in later material. Cole and Jakimik point out that this finding is compatible with a model in which word-initial material plays a special role in lexical access.

\begin{abstract}
According to this view, reaction times are faster to mispronounced second syllables because the intended word has been accessed as one of a set of word candidates from information in its (correct) first syllable. . . By the same reasoning, a mispronounced first syllable provides misleading information, since the listener will access word candidates beginning with the mispronounced syllable. ... We assume that the subject initiates a [mispronunciation-]detection response when the second syllable fails to confirm any of the word candidates suggested by the first syllable. Thus, ... a mispronunciation in the first syllable of a word cannot be detected until information from a subsequent syllable is available. (Cole \& Jakimik 1980:968; original emphasis)
\end{abstract}

Finally, there is evidence from semantic priming studies indicating that a match in word-initial material is more likely to activate a lexical entry than a match in later material. As noted above, semantic priming is a phenomenon in which prior exposure to one word, such as captain, facilitates the recognition of a semantically related target word, such as boat. That is, the response time to a lexical decision task ("word or nonword?") for a semantically primed target is faster than that to a control target that was preceded by a unrelated, non-priming word.

Marslen-Wilson, Brown, \& Zwitserlood (1989) and Zwitserlood (1989) show that presentation of a phonetic string activates multiple lexical entries whose initial portions are compatible with it. For example, when (Dutch-speaking) listeners were presented with the stimulus [kapi:], which corresponds to the initial portion of two words, kapitein 'captain' and kapitaal 'capital,' they showed semantic priming effects for both boot 'boat' and geld 'money'; this indicates that both kapitein and kapitaal were lexically activated by the string [kapi:]. 
Marslen-Wilson and Zwitserlood (1989) then examine the effect of semantic priming for what they call rhyme primes-words (and nonwords) that are identical to an intended prime word except in the initial segment. Their results show that semantic priming by a rhyme prime, such as woning 'dwelling' (tested for its priming effect on the target word bij 'bee,' which would be primed by the intended rhyming word honing 'honey'), was less effective than priming by the intended lexical form (such as honing itself) or by an initial partial prime (as in the case of [kapi:] discussed above). Crucially, this was true even in the case of three-syllable rhyme primes, in which the degree of segmental overlap was even greater than that between [kapi:] and kapitein or kapitaal. Marslen-Wilson and Zwitserlood (1989) conclude that a match in initial material is more important in activating a lexical entry than simply the amount of total match across the whole of the form.

The results reported by Zwitserlood and colleagues are further supported by the findings of Pitt \& Samuel (1995) that "beheading" words, i.e., removing their initial onsets, slows recognition. Pitt \& Samuel (1995) first demonstrate that response time for a phoneme-monitoring task is faster for words than for nonwords in a condition where the uniqueness point (the point at which the word can be uniquely identified, or the nonword diverges from all possible words) comes early in the word or nonword. That is, successful word recognition at an early point gives the listener access to the lexical entry for that word, which facilitates phoneme monitoring. Listeners know that the target phoneme is part of the word perhaps even before they hear it, so they can respond quickly when they do hear it; but nonwords have no lexical entry, so there is no analogous effect.

However, the response-time advantage for words over nonwords disappeared when the words and nonwords had their initial onsets removed. The removal of the initial onset of a word therefore seems to inhibit word recognition, even when (as in the rhyme-prime study of Marslen-Wilson \& Zwitserlood 1989) there is otherwise a good match between the stimulus and the non-initial portion of the target lexical entry.

In conclusion, these results show that word-initial material is paid particular attention in speech perception ( $\$ 4.3 .2 .1)$, and more specifically that word-initial material has a particularly strong influence on how well a given lexical entry is deemed to match the acoustic input during early-stage word recognition, as seen in the results of experiments that test whether or how easily certain lexical entries are 
activated by a given acoustic input $(\$ 4.3 .2 .2){ }^{38}$ Thus, the initial syllable is legitimately included in the set of psycholinguistically strong positions. ${ }^{39}$

38. The importance of initial material in early-stage word recognition has been given different implementations in different models of speech processing. For example, in the Search model (Forster 1976, 1990), the first few phonemes from the acoustic input function as an access code that activates all lexical entries with a matching initial portion. In the Cohort model (Marslen-Wilson \& Welsh 1978; Marslen-Wilson 1984, 1987), the first few segments of the word are used to retrieve a 'cohort' of possible word-candidates from the lexicon with matching initial segments; the cohort is then narrowed down to one word through the use of syntactic, semantic, and further phonetic or phonological information. In interactive activation models such as TRACE (McClelland \& Elman 1986), there is no initial retrieval of a set of word-candidates based on the first few segments; instead, all lexical entries that are sufficiently similar to the auditory input become partially activated and compete with each other. In models such as these, the special importance of word-initial material may be incorporated by allowing a word-initial match to enhance activation levels more than a match in later positions in the word, as has been suggested for a TRACE-type model by Marslen-Wilson (1987) and Marslen-Wilson \& Zwitserlood (1989).

39. Most of the psycholinguistic results discussed in this section indicate that the first few segments of a word are important in recognition, but they do not necessarily point to the initial syllable as a unit. Nevertheless, the positional-neutralization patterns examined by Beckman $(1995,1997,1998)$ show privileged behavior in the initial syllable as opposed to other syllables, indicating that it is indeed a syllable-sized unit that is a phonologically strong position. In fact, there is evidence that syllables are used in processing as a basic perceptual unit, at least in some languages (see, e.g., Mehler, Dommergues, Frauenfelder, \& Segui 1981; Segui, Dupoux, \& Mehler 1990), although Cutler, Mehler, Norris, \& Segui (1986) argue that syllables are not used as basic units for processing by native listeners of English.

If the syllable is available as a basic perceptual unit in the processing of spoken language, then it is not surprising that the initial syllable would be treated as a strong position on the basis of the importance of initial material. (Segui, Dupoux, and Mehler (1990), for example, explicitly propose that early-stage word recognition in French is based on the initial syllable). And even if the syllable is not a universally basic unit of perception, it may well be that the grammar gives the special status of a strong position to the entire initial syllable because it is the single grammatical constituent that best matches the psycholinguistically relevant "first few segments."

Casali $(1996,1997)$ actually does propose positional faithfulness constraints for initial segments, but since he is looking at positional faithfulness effects specifically in word-initial vowels, his findings are consistent with initial-syllable faithfulness. 


\subsubsection{The importance of roots in early-stage word recognition}

The morphological root has been identified as a strong position on the basis of its ability to resist phonological processes and to maintain greater numbers of phonological contrasts than affixes can have (see, e.g., McCarthy \& Prince 1995; Alderete 1999b, 2001; Urbanczyk 1996ab, 1999b; Pater 1996, 1999; Beckman 1998; Struijke 1998, 2000).

This section reviews evidence that roots qualify for inclusion in the set of psycholinguistically strong positions because they are important in early-stage word recognition. ${ }^{40}$ Roots are shown to affect word recognition in inflectionally complex forms (\$4.3.3.1), and, crucially, even in derivationally complex forms (\$4.3.3.2). The latter result is somewhat surprising, given a widely held view of the structure of the lexicon in which derivationally complex forms have their own lexical entries (Chomsky 1970; Halle 1973; Aronoff 1976; Scalise 1986). This view of the lexicon is supported in that derivationally complex forms often have idiosyncratic, noncompositional meanings; derivational affixes are often less than fully productive; and native speakers are able to distinguish between "actual complex words" and "possible but non-occurring complex words." Nevertheless, the psycholinguistic evidence reviewed in \$4.3.3.2 indicates that roots play an important role in earlystage word recognition even for derivationally complex forms.

These results support the proposal that roots play a fundamental role in determining how lexical entries are structured and thus in how morphologically complex lexical entries are accessed during early-stage word recognition.

\subsubsection{Processing of words with inflectional affixes}

Roots have been shown to be much more involved than inflectional affixes in earlystage word recognition. For one thing, it is known that various aspects of language processing are sensitive to word frequency, but several studies have indicated that,

40. The extent to which information about the complete morphological structure of a word is relevant at prelexical stages of processing - and therefore the extent to which it is relevant for word recognition -is somewhat controversial (see, e.g., Henderson (1985) and Hawkins $\&$ Cutler (1988) for reviews of the literature). Furthermore, several of the experiments discussed below are visual rather than auditory experiments, and not all results from visual experiments may be directly applicable to theories of auditory processing. (It is clearly indicated for each experiment below whether or not it was auditory.) Despite these complications, there is a reasonable amount of evidence that roots are important in the recognition of morphologically complex words. 
for an inflected form of a particular root, it is the frequency of the root itself or of the whole set of inflected forms that is relevant, rather than the frequency of the individual inflected form. Rosenberg, Coyle, \& Porter (1966) ran an experiment in which they presented visual lists including English -ly adverbs and then asked participants to recall the presented items. Rosenberg et al. found that adverbs formed from high-frequency adjective roots were more likely to be recalled than adverbs formed from low-frequency adjective roots, even though the adverbs themselves were all low-frequency words. Compatible results for nouns have been found by Baayen and Schreuder and their colleagues: Baayen, Dijkstra, \& Schreuder (1997) and Baayen, Burani, \& Schreuder (1996) show, for Dutch and Italian respectively, that visual lexical decision times for basic, uninflected nouns are affected by the frequency with which all inflected forms of those nouns occur, not by the frequency of the bare or basic form alone. The results of these three studies show that, for aspects of processing that are sensitive to frequency of occurrence, all inflected forms of a given root are considered tokens of the same item.

Other studies further support the claim that in early-stage word recognition, inflectional affixes are not considered an integral part of the word to be recognized (or at least play a subordinate role to that of roots). A study by van der Molen \& Morton (1979) showed that nouns and their plural morphemes were sometimes recalled separately in a visual memory task. Participants were presented with lists of six words each, and were asked to recall the contents of each list. Every list included singular nouns; some lists included plural nouns as well. In $17 \%$ of the cases in which nouns were recalled that had been plural in the lists, they were incorrectly recalled in the singular. By contrast, there were extremely few instances of singular nouns being incorrectly recalled as plural forms, with one interesting exception: spurious plurals did occur in cases where a plural noun from the same list had been incorrectly recalled (forgotten entirely or recalled as a singular noun), as though such errors had given rise to a "loose" plural morpheme in memory. van der Molen \& Morton (1979) thus conclude that the nouns and the plural morphemes were given somewhat independent representations in memory during the recall task.

Jarvella \& Meijers (1983), in an auditory experiment, found a similar separation between verb roots and inflectional morphology in Dutch, a result which is particularly interesting in that some of the inflectional affixes in their study were prefixes rather than suffixes (past-participial ge- on two of the three classes of verbs). Listeners were presented with a verb in either a past or past-participle form, and had to identify whether the verb that came at the end of a subsequent string of words was the same or different with respect to either the root or the inflectional form. Jarvella \& Meijers found that both "same" and "different" responses were faster for the dimension "root" than for the dimension "form," even when the two 
verbs given were different forms of the same root. Interestingly, the advantage for "root" over "form" responses was found even in cases where decisions about form involved prefixes and so were in principle possible to make even before the roots themselves could have been recognized. These results suggest that the recognition of a verb root is faster or easier than the recognition of an inflectional affix, and are compatible with a theory in which inflected forms are accessible only if the root is accessed first. ${ }^{41}$

Results from auditory and visual experiments on inflected forms in SerboCroatian have been used to argue for a "satellite model" of lexical organization (Lukatela, Gligorijevic, Kostić, and Turvey 1980), in which the oblique-case forms of nouns are accessed through the nominative form. Lukatela et al. (1980) present results from a visual lexical-decision task showing that response times to all oblique case forms of a particular noun are the same, even when the individual frequencies among oblique cases differ (i.e., the genitive is typically much more frequent than the instrumental). Furthermore, the response times to the oblique forms are slower than to the nominative form, again, even when certain oblique forms are no less frequent than the nominative form. These original results have been replicated, again in Serbo-Croatian, by Katz, Boyce, Goldstein, \& Lukatela (1987) in an auditory study and by Lukatela, Carello, \& Turvey (1987) in a visual study that included nouns whose root shape changes in some but not all oblique forms. While in Serbo-Croatian the form with special status in early-stage word recognition is the nominative form, which does not always correspond exactly to the morphological root (as in [frula] 'flute-NOM' from the bound root /frul-/), the evidence that supports the satellite model does at least show that all the inflectional forms of a given root are stored together and cannot be accessed independently.

Finally, there are studies showing that inflected forms of a root are able to prime the bare or basic form of the root, in many cases to the same extent as "identity priming" of the root by a prior occurrence of the root itself. Stanners, Neiser, Hernon, \& Hall (1979), in a visual lexical-decision task using English verb roots, found that regularly inflected verb forms (such as pours or poured) are as effective at priming the bare root pour as is a prior presentation of pour itself. They found that irregularly inflected verbs (i.e., hung as a prime for hang) also showed significant priming effects, although not as much as the regularly inflected forms. But even the partial priming effect observed by Stanners et al. for irregular inflections was, they state, "larger and more robust" than the effect of ordinary

41. However, it will be seen in $\S 4.3 .4$ that non-initial stressed syllables have been shown to cause spurious lexical-access attempts in Dutch. Since the inflectional prefix ge- is unstressed, there may be a stress-related confound in these results. 
semantic priming between morphologically unrelated words (such as captain and boat or bee and honey).

Fowler, Napps, \& Feldman (1985) argue that the difference found by Stanners, Neiser, Hernon, \& Hall (1979) between regular and irregular inflected forms was an artifact of their experimental design. Fowler et al. (1985) used Stanners et al.'s (1979) design and replicated their results, but then, using a modified design that allowed more words to intervene between the prime and the target to reduce the effects of episodic priming (i.e., the participant's actually remembering a prior stimulus-response pair), they found equivalent priming of roots by regular and irregular inflected forms. (Similarly, Jarvella \& Meijers (1983) found no difference in the response times for "stem" judgments between strong ("irregular") and weak ("regular") verbs in Dutch.) Fowler et al. (1985) also replicated their results in an auditory version of their study, again finding statistically full priming of roots - and in this study, of complex forms as well-by regular and irregular inflected forms.

Feldman \& Moskovljević (1987) found similar results for Serbo-Croatian, namely, that a dative/locative form is primed by the nominative (basic) form just as much as by itself. They found significant priming of the dative/locative by the instrumental form as well, but in this case the priming was significantly less than by the nominative or in the identity-priming case. (This discrepancy is reminiscent of Lukatela et al.'s "satellite model" of the Serbo-Croatian lexicon outlined above, in that the oblique forms of a root may only be able to interact with each other through the mediation of the nominative form.) Feldman and Moskovljević were able to show that words with orthographic and phonological overlap but no morphological relationship had no priming effect, so even if the priming of one oblique form by another is only partial, it is still noteworthy.

Additionally, Reid \& Marslen-Wilson (2000) demonstrate with data from Polish that different members of a noun or verb inflectional paradigm prime each other, even when the root portion of a complex form differs from the basic form of the root as a result of phonological or morphophonemic alternations.

In summary, there is evidence from a number of different languages that, in a word containing a root and inflectional affixes, the root is processed separately from the affixes. Furthermore, forms with different inflectional affixes often behave like tokens of the same form with respect to frequency-sensitive phenomena and priming effects. Finally, inflected words appear to be processed as though they are organized around their roots. Thus, compared to inflectional affixes, roots have a privileged status in the structural organization of the lexicon and thus in early-stage word recognition-which seems appropriate, given that the major semantic content of an inflected word is contributed by the root morpheme. 


\subsubsection{Processing of words with derivational affixes}

Many studies involving derivational affixes, like those involving inflectional affixes, show a special role for roots in early-stage word recognition. In some cases, the derivational affixes themselves give rise to priming effects; as might be expected, they also seem to add complexity to the processing of words. Nevertheless, in most studies, roots are still seen to have an importance equal to or greater than that of derivational affixes.

There is evidence that roots inside derivationally complex words have priming effects, just as they do in forms with inflectional morphemes. Stanners, Neiser, Hernon, \& Hall (1979), in the visual study discussed in \$4.3.3.1, found that adjectival and nominal derivatives of English verbs significantly prime their verb roots, but not as much as the roots themselves or regular inflectional forms do. However, as noted above, Fowler, Napps, \& Feldman (1985) take issue with the experimental design used in the Stanners et al. study; once again, with their modified design, Fowler et al. found that morphological derivatives were able to prime their roots to an extent that was not significantly different from identity priming by the roots themselves, in both visual and auditory experiments.

Stanners, Neiser, \& Painton (1979) did a series of experiments involving derivational prefixes. They found only partial priming of target words with bound roots (such as revive or progress) when the roots and prefixes were presented separately (i.e., combined with other prefixes or roots respectively) ahead of time. However, there seem to be some problems with these experiments. First, Stanners, Neiser, \& Painton (1979) did not attempt to factor out the separate effects of priming contributed by the root and the prefix, since both were presented in each case. Also, the distance between targets and primes in these experiments was only 8-12 items, so presumably the criticisms raised by Fowler et al. (1985) about the confounding effects of episodic priming in Stanners, Neiser, Hernon, \& Hall's (1979) experiments are valid here as well.

The fourth experiment in Stanners, Neiser, \& Painton (1979) was different in several respects. First, the prefixed forms involved free roots with productive prefixes like un- and re-. Second, the distance between prime and target was increased to an average of 31 intervening items, which is more like the experimental design used by Fowler et al. (1985). Finally, Stanners et al. used the prefixed form as a prime and looked for priming effects on the bare root, rather than looking for priming effects on a complex form by a pair of other complex forms. In this experiment, they actually did find full priming of bare roots by prefixed forms: the degree of priming by prefixed forms did not differ significantly from identity priming by the roots themselves. 
Evidence that roots are primed by derivationally complex forms has been found in other languages as well. Feldman \& Moskovljević (1987) found priming of roots by derived forms in Serbo-Croatian. Boudelaa \& Marslen-Wilson (2000) report priming effects in Arabic between words that share the same morphological root even when they are not closely semantically related, such as mudaaxalatun 'conference' and duxuulun 'entering,' which share the root /dx1/.

There is a certain amount of evidence suggesting that derivational prefixes are removed or ignored to allow processing of roots first, an operation dubbed "prefixstripping" by M. Taft \& Forster (1975). For example, M. Taft, Hambly, \& Kinoshita (1986), in both auditory and visual experiments, compared lexicaldecision response times to four kinds of nonwords: real (bound) stems with real, but inappropriate, prefixes (dejoice), real stems with non-prefixes (tejoice), non-stems with real prefixes (dejouse), and non-stems with non-prefixes (tejouse). They found that the prefixed forms were more difficult than the non-prefix forms overall (having both slower response times and higher error rates). But crucially, the prefixed forms were more difficult to reject for stems than for non-stems, while the non-prefix forms were rejected just as quickly and accurately whether they contained real stems or not. In other words, there is no evidence that the nonwords that started with non-prefix strings were decomposed, so the fact that some of them contained real stems was apparently irrelevant to the processor. However, a difference between stem and non-stem forms appeared in the nonwords that started with real prefixes. In this case, it appears that the processor may have used the presence of the prefix as a cue to look inside the word for evidence of a stem. ${ }^{42}$

In earlier work, M. Taft (1979) also found root-frequency effects for prefixed words containing bound roots. For example, he found that participants were able to recognize deploy more quickly than deflate, even though the two words have the same frequency of occurrence, because -ploy has an overall frequency that is higher than that of -flate (compare employ and inflate). Bradley (1980) found rootfrequency effects for suffixed forms if the suffixes were productive and semantically transparent, like -ness, -ment, and -er (agentive).

Thus, while the available evidence is not absolutely conclusive, because of possible confounding effects in certain experiments stemming from the use of visual rather than auditory stimuli, the lack of a detailed comparison of inflectional with derivational prefixes, and the separate effects of stress pattern on lexical access in some languages, there is evidence that roots have a special role even in the

42. However, there is a possibility of interference from the effects of stress, since the stress would fall on the stem, and stressed syllables have been shown to initiate lexical access attempts in English (see \$4.3.4.1). 
processing of words with derivational morphology. Root-priming effects and rootfrequency effects are found with derived forms just as for inflected forms, and experimental participants appear to search for roots inside prefixed forms.

However, there is evidence that derivational morphology is somewhat more important in early-stage word recognition or word-level processing than inflectional morphology; again, this seems intuitively reasonable, given the greater semantic contribution of derivational morphology and the fact that derived forms are probably lexically listed. For example, some results indicate that extra complexity is introduced by derivational structure. Manelis \& Tharp (1977) had experimental participants view pairs of stimuli and give a positive response only if both were words. The stimuli all ended in letter sequences that could be suffixes, and included real words, either monomorphemic (somber) or bimorphemic (dark+er), and three classes of nonwords: C[ontrol] (locter), W[ord] (desker), and F[ragment] (garmer, cf. garment). They found that when both stimuli were words, response times were shorter when both words were either simple or complex, but longer if the pair was mixed. They also found that both the $\mathrm{W}$ and $\mathrm{F}$ nonwords were rejected more slowly than the controls. Jarvella \& Meijers (1983), in the Dutch verb experiment described above, found that response times for the class of verbs that start with a derivational (inseparable) prefix were slower than for the other classes of verbs. These results-as well as those from M. Taft et al. (1986) reported above, where responses to prefixed nonwords were slower than those with non-prefixes-all indicate that derivational morphological structure may add complexity to word recognition.

There are also psycholinguistic results suggesting that at least some derivationally related words have separate lexical entries rather than being grouped together as part of the entry of the root. Schreuder \& Baayen (1997), in a visual experiment measuring lexical-decision times to Dutch nouns, found an effect of the combined frequency of inflected forms of a noun (see also §3.4.3.1), but the total frequency of the "morphological family," including derived forms and compounds containing the noun in question, had no effect. Bradley (1980) found a difference between semantically transparent and opaque derivational affixes in English; while root frequency affected forms with transparent suffixes like -ment or -ness (as noted above), there was no effect of root frequency on forms with opaque, nonproductive affixes like -(t)ion, suggesting that the latter words have their own lexical entries.

Finally, there is evidence that derivational affixes can have priming effects. Boudelaa \& Marslen-Wilson (2000) show that, in Arabic, forms with two different roots but the same "word-form" (i.e., binyan or morphological template) prime each other as long as the word-form makes a semantic contribution in each case. For

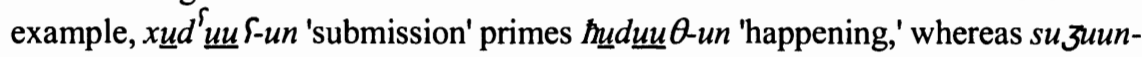
un 'prisons,' which contains the same vowel pattern but is semantically unrelated, 
does not. (However, Boudelaa \& Marslen-Wilson (2000) also cite studies by Frost and Deutsch and colleagues reporting that word-form has no priming effect in closely related Hebrew.)

Nevertheless, taken together, these results do not indicate that derivational affixes play a more significant part in word recognition than roots, although some of them suggest that derivational affixes may contribute links among lexical entries or otherwise affect the way that the lexicon is structured. ${ }^{43}$

Thus, the psycholinguistic findings reviewed in this section support the proposals made above about psycholinguistically strong positions. Namely, they show that roots and initial syllables are important in early-stage word recognition in a way that other positions, particularly stressed syllables (see \$4.3.4) and derivational affixes, are not. Therefore, these two positions can be grouped together as the set of psycholinguistically strong positions, crucially defined in terms of their special role in early-stage word recognition.

\subsubsection{Absence of a role for prosodic properties in early-stage word recognition}

The Segmental Contrast Condition has been proposed as a constraint filter that restricts $\mathbf{M} / \Psi_{\text {str }}$ constraints to those whose satisfaction does not entail the neutralization of segmental contrasts in psycholinguistically strong positions (aside from the special case of $\sigma_{1}$ left-edge demarcation effects; see $\S 4.3 .5$ ). This filter correctly predicts the relatively small inventory of augmentation constraints that can be relativized to the psycholinguistically strong positions initial syllable and root, especially compared with the large number of attested augmentation constraints for the strong position stressed syllable, which is comparable in size.

This subsection reviews psycholinguistic evidence bearing on the role of the prosodic properties stress and tone in speech perception. The findings presented here motivate two of the characteristics of the Segmental Contrast Condition as

43. Revithiadou (1999ab) has proposed, based on accentual phenomena, that if a derivational affix is the head of a word, it can have the status of a strong position. However, there is an alternative account of these accentual phenomena, with in fact broader empirical coverage (Alderete 1999b), and derivational heads have not been convincingly shown to behave as strong positions with respect to contrasts outside the domain of stress and accent. There also seem to be no examples of augmentation in derivational heads. Therefore, derivational heads have not been included in the set of strong positions here-a decision that is further supported by the psycholinguistic evidence reviewed in this section, which does not indicate that derivational affixes play a primary role in early-stage word recognition. 
formulated above. First, the fact that this filter applies to augmentation constraints relativized to roots and initial syllables but not to those for stressed syllables-more generally, the proposal that the stressed syllable is not included in the set of psycholinguistically strong positions-is supported by the findings in \$4.3.4.1: while the stressed syllable is involved in certain aspects of speech perception (\$4.3.4.1.1), its major role is in the segmentation of the speech stream rather than in accessing lexical entries directly (\$4.3.4.1.2); furthermore, this use of the stressed syllable in speech segmentation is language-specific rather than universal, since it depends on the system of stress placement in a given language (\$4.3.4.1.3). Thus, the stressed syllable is not as fundamentally relevant for early-stage word recognition as are the initial syllable and the root.

Another correct prediction made by the Segmental Contrast Condition as formulated above is that $\mathbf{M} / \Psi$ str constraints referring to prosodic properties, such as HAVESTRESS/Root, are attested. The second goal of this subsection is to provide psycholinguistic evidence motivating the Segmental Contrast Condition's distinction between segmental and prosodic properties with respect to augmentation constraints for psycholinguistically strong positions. The evidence reviewed here shows that this distinction is justified because prosodic properties such as stress (§4.3.4.1) do not play the same role in early-stage word recognition that is played by segmental properties. That is, stress is used in later stages of word recognition, to confirm which lexical entry should be selected out of those that are initially activated by the auditory input. However, mismatches in stress do not prevent the early-stage activation of a lexical entry for comparison with the auditory input, even in languages where stress is lexically contrastive. The case of tonal contrast is considered in \$4.3.4.2. Here, the psycholinguistic results are few and somewhat tentative, but there is at least some evidence that tonal contrasts behave differently from segmental contrasts in speech perception, and thus that HTONE/ $\mathbf{\Psi s t r}$ constraints may pass the SCC.

\subsubsection{The role of stressed syllables in speech perception}

To support the claim stated above, that the stressed syllable is not fundamentally relevant in early-stage word recognition in the way that the initial syllable and the root are relevant, requires some discussion. There is a fair amount of evidence that stressed syllables actually are important in certain aspects of speech perception and processing, particularly in stress-timed languages such as English and Dutch (§4.3.4.1.1). However, there is also evidence that stress location and stress-related contrasts are not fully utilized in early-stage word recognition (\$4.3.4.1.2). Following explicit or implicit proposals by L. Taft (1984), Norris, McQueen, \& 
Cutler (1995), and Mattys \& Samuel (2000), an argument is presented ( $\$ 4.3 .4 .1 .3)$ that the role of the stressed syllable in early-stage word recognition is only an indirect and language-particular one. Stressed syllables are indeed utilized in certain languages during speech processing, but they are used mainly to help locate initial syllables, not to access the lexicon itself. Thus, the stressed syllable is not a psycholinguistically strong position, so $\mathbf{M} / \sigma$ o constraints are not subject to filtering by the Segmental Contrast Condition.

\subsection{How stressed syllables are involved in processing}

A number of researchers have observed that stressed syllables appear to have some kind of special status in speech processing. This section reviews these results.

Cole \& Jakimik $(1976,1980)$ show that listeners are better able to detect mispronunciations (in running speech) when they occur in stressed syllables. However, this result might simply reflect the fact that stressed syllables tend to be uttered more distinctly than unstressed syllables, and need not be related to word recognition per se.

Brown \& McNeill (1966), in their study of the "tip-of-the-tongue" state, found some provisional evidence that it is possible to recall the stress pattern of the target word while in a TOT state. They examined the words offered by the experimental participants as "sounding similar" to the word they were trying to recall, and found that these words had a tendency to share stress with the target words. However, for various statistical reasons, they were only able to investigate the disyllabic words in their materials, which were only 31 out of 233 words. Brown \& McNeill (1966:330) can conclude only that "we are left suspecting that [the participant] in a TOT state has knowledge of the stress pattern of the target, but we are not sure of it."

Nakatani \& Schaffer (1978), in an experiment using "reiterant speech" (i.e., speech in which a single syllable, in this case [ma], is repeated over and over to replace the original segmental content of a phrase), found evidence that listeners used prosodic information to locate word boundaries. Reiterant speech was used to mimic adjective+noun phrases of the shape [mama\#ma] or [ma\#mama], with various stress patterns. Certain of their listeners were able to use their knowledge of possible versus impossible stress patterns to locate word-boundaries; in particular, when two primary-stress syllables were adjacent, these participants placed the word boundary between them.

There is evidence that stressed syllables (primary or secondary), when they occur in non-initial position, give rise to spurious lexical-access attempts. Cutler \& Norris (1988) report the results of experiments in which (English-speaking) 
listeners were asked to detect real words embedded inside longer nonwords. They found that listeners were able to detect the real word thin in both thintef [' $\theta$ Intəf] and thintayf ['OIn,tejf], but that mint was detected much more easily in mintef ['mintəf] than in mintayf ['mIn,tejf]. They conclude that the presence of the secondary stress in ['mIn,tejf] causes segmentation of the speech stream, and a new attempt at lexical access, at the phone [t], and this impedes recognition of the word mint. (The word thin, on the other hand, is complete before the beginning of the second stressed syllable in [' $\theta$ In,tejf], so recognition of this word is not impaired.) ${ }^{44}$ These findings have been replicated by Norris, McQueen, \& Cutler (1995) for English and by Vroomen \& de Gelder (1995) for Dutch.

Luce \& Cluff (1998) report additional evidence, from a semantic priming study, that non-initial stressed syllables cause spurious lexical-access attempts in English. They used words like hemlock, that had two heavy syllables, where the second syllable was homophonous to an unrelated monosyllabic word (i.e., lock), and where gating studies showed that the original disyllabic word would be recognized before its offset. They found that, e.g., hemlock would semantically prime a word related to lock but unrelated to hemlock, such as key. This result indicates that a new access attempt was initiated at the second stressed syllable in these words.

Another source of evidence that speakers of English and Dutch make use of stressed syllables in processing comes from misperceptions involving word boundaries. L. Taft (1984) found that both an iambic sequence (i.e., W(eak)S(trong)) that is ambiguous between a one-word and a two-word interpretation (in \# 'vests versus in'vests), and an iambic nonsense word, are more likely to be given a two-word interpretation than a trochaic sequence (SW) would be. Cutler \& Butterfield (1992) investigated a corpus of natural misperceptions and also

44. One might argue that the results reported by Cutler \& Norris (1988) could have another explanation: the [t] in mintef is more likely to be syllabified with the preceding syllable than is the [t] in mintayf, because of the different stress patterns of the two nonwords, and it might be this difference rather than the initiation of a lexical-access attempt that makes it harder to perceive mint in mintayf than in mintef. However, results from experiments by Cutler, Mehler, Norris, \& Segui (1986) show that the difference in syllabification between ba.lance and bal.cony does not affect English-speaking listeners' ability to recognize the substrings $b a$ or $b a l$ in these words (whereas for French-speaking listeners, the difference between ba.lance and bal.con has an effect). While the Cutler et al. (1986) task involved the spotting of syllable-sized nonword sequences, not the spotting of entire words as in the Cutler \& Norris (1988) experiment, Cutler et al.'s results suggest that dividing a target between two syllables does not necessarily make that target more difficult for English speakers to find. 
produced misperceptions in the laboratory by having participants listen to faint speech and report what they thought they heard. Their results from the two investigations show that the insertion of a word boundary is more common than boundary deletion before a stressed syllable, and that boundary deletion errors are more common than boundary insertion errors before an unstressed syllable. The faint-speech results were replicated by Vroomen \& de Gelder (1995) for Dutch; Bond (1999) reports analogous findings from a different corpus of naturally occurring English misperceptions.

Finally, there is evidence that words that start with unstressed syllables, or perhaps non-main-stress syllables, are more difficult to process in English. Cutler $\&$ Clifton (1984) found that lexical decision response times for SW words were faster than those for WS words. Gow \& Gordon (1993) report that both initial and final syllables were detected faster in trochaic forms than in iambic forms. Similarly, Mattys \& Samuel (2000) found that words that do not begin with a primary stress result in a processing delay and create more of a memory load (i.e., cause poorer performance on another task being carried out simultaneously) than words with initial primary stress. A somewhat related finding is reported, also for English, by L. Taft (1984) and Cutler \& Clifton (1984): when SW words are mispronounced as WS, response time is slowed. However, when WS words are mispronounced as SW, this either has no effect on response time (Cutler \& Clifton 1984) or even reduces response time (Taft 1984).

\subsection{How stressed syllables are not involved in processing}

The evidence presented above shows that stressed syllables are somehow relevant for speech processing, at least in English and Dutch. However, there is evidence that the stress pattern, or the location of main stress in a word, is not relevant in early-stage word recognition. Most strikingly, knowledge of the stress pattern of a word seems not to prevent the activation of a lexical entry that has a different stress pattern but the same segmental content.

First of all, it is important to factor out the effects of sentence-level stress when investigating the role of word stress in processing. Shields, McHugh, \& Martin (1974) found that the presence of sentential stress (i.e., a pitch accent) decreased response time for phoneme monitoring (in nonwords), but that when the experimental nonwords were cut from their sentential contexts and spliced into lists of nonwords, mere word-level stress had no effect on response time. This result also raises the question of whether Cole \& Jakimik's $(1976,1980)$ findings, that mispronunciations in running speech were better detected in stressed syllables, were caused by sentential stress rather than word stress. It has been known since work by 
Cutler (1976) that sentential focus (as signaled by preceding intonational contour) facilitates the processing of a word, but it may be only that sentence stress draws the hearer's attention to a word for semantic or pragmatic reasons, and this increased attention is responsible for the faster phoneme-monitoring and mispronunciationdetection times reported by Shields, McHugh, \& Martin (1974) and Cole \& Jakimik $(1976,1980)$.

Cole \& Jakimik (1980) provide a further reason to discard the hypothesis that early-stage word recognition proceeds directly by means of stressed syllables. They note:

The hypothesis that word candidates are accessed from stressed syllables did not fare well in the present experiment. According to this hypothesis, we should observe faster reaction times to words with intact stressed syllables than to words with mispronounced stressed syllables, since only words with intact stressed syllables will produce word candidates which include the intended (but mispronounced) word. (Cole \& Jakimik 1980:968)

Cutler \& Clifton (1984) and Cutler (1986) report findings that rather strongly discredit the possibility of a direct relationship between stressed syllables and earlystage word recognition. Cutler \& Clifton's (1984) results show that knowing the stress pattern of a word in advance does not make response time any faster in a lexical decision task. Cutler (1986) found that each member of a pair like for'bear/'fore,bear, homophones that differ only in stress (crucially, not in vowel quality), lexically activates the other; presentation of forbear shows semantic priming effects for words that are semantically related to forebear, and vice-versa. This indicates that a mismatch in stress pattern is not enough to keep a word from being lexically activated when its segmental content matches that of the auditory input being processed, or, in Cutler's (1986) terms, that lexical prosody does not constrain lexical access. ${ }^{45}$

45. A recently published paper by Cutler \& van Donselaar (2001) shows that in Dutch, a stress mismatch does reduce the activation of candidates for word-recognition to some degree-words differing only in stress contour do not show exactly the same patterns of lexical activation, so they are not functionally homophones in early-stage word recognition in the way that English 'fore, bear for'bear are. However, Cutler and van Donselaar still found that a segmental mismatch had a much larger effect than a stress mismatch in removing word candidates from consideration. Thus, the distinction made by the Segmental Contrast Condition may not be between contrasts that are relevant and not relevant in word recognition, but between contrasts that are relevant to a certain degree and less relevant than that degree. Crucially, segmental properties and stress still differ in their importance. 


\subsection{Stress-based segmentation as a language-particular processing strategy}

The results presented thus far indicate two rather different patterns. On the one hand, in Dutch and English, stressed syllables have been shown to affect processing, because (a) they trigger lexical-access attempts even when they appear wordmedially (as in hemlock) and (b) words that do not start with stressed syllables are more difficult to process. On the other hand, stress patterns are not utilized in earlystage word recognition, because (a) prior knowledge of the stress pattern does not facilitate word recognition and (b) a mismatch in stress pattern is not important if segmental material matches.

But these two sets of facts do not appear contradictory once it is understood that recognizing words in continuous speech actually involves two distinct operations. One operation, of course, is word recognition itself: the acoustic/phonetic signal must be matched to entries in the lexicon so that syntactic and semantic information becomes available for sentence processing. But another operation that must be performed is that of (word) segmentation-before words can be recognized, the continuous speech signal must be divided into words.

As noted by many researchers, including L. Taft (1984), Cutler \& Norris (1988), and Norris, McQueen, \& Cutler (1995), identifying the beginnings of words in the speech stream is not a trivial task. It is not even possible to assume that every word starts where a previous word ends, because some words are contained inside longer words (such as fan in fantastic), and furthermore hearers continue to understand speech even after encountering a word that they do not know or that is unintelligible, such they might not know where that word would end.

Taft (1984), Cutler \& Carter (1987), and Vroomen \& de Gelder (1995) note that in English and Dutch, words are likely to have initial stress. Cutler \& Carter (1987) compiled statistics from a 33,000-word computer-readable English dictionary and found that words with initial primary or secondary stress (including monosyllables) made up $73 \%$ of the words listed. They next examined a 190,000 word natural-speech British English corpus, and found that over $70 \%$ of the lexical words had initial primary or secondary stress. Moreover, they found that over threefourths of the strong syllables in the corpus were the initial (or only) syllables of lexical words, whereas two-thirds of the weak syllables were the initial (or only) syllables of function words. Vroomen \& de Gelder (1995), using the CELEX database, found that only $18.6 \%$ of English lexical words start with weak syllables. For Dutch, they found that only $12.3 \%$ of lexical words had (invariant) initial weak syllables, and of those, $96 \%$ began with the prefixes be-, ge-, or ver-.

Thus, in both English and Dutch, a relatively efficient processing strategy for finding the onset of a lexical word is to go to where the stressed syllables are. This 
insight is the basis for the Salience to Onset segmentation strategy of Taft (1984) and the Metrical Segmentation Strategy (MSS) of Cutler \& Norris (1988) and Norris, McQueen, \& Cutler (1995), for English, as well as the Syllable-Based Segmentation Strategy of Vroomen \& de Gelder (1995) for Dutch (a modification of the MSS). While these proposals differ in certain details, they all essentially claim that segmentation of the speech stream, in these languages, takes place when a stressed syllable is encountered. Since segmentation is a precursor to word recognition, these processing strategies are in effect assuming that stressed syllables are initial syllables. ${ }^{46}$ But the role of stressed syllables in early-stage word recognition is not a direct one; it is indirect, through the use of stressed syllables for segmentation.

Further reason to believe that stressed syllables do not have a universal, basic role in early-stage word recognition comes from considerations of stress placement cross-linguistically. Hyman's (1977) survey of stress patterns, containing about 400 languages, includes the following statistics:

(76) Cross-linguistic stress patterns (Hyman 1977)

$\begin{array}{lc}\text { Dominant initial stress } & 114 \\ \text { Dominant penultimate stress } & 77 \\ \text { Dominant final stress } & 97\end{array}$

No dominant stress placement $\quad 113$

The first three figures include languages with predictable stress on the position indicated as well as languages that simply show a majority of words with stress on that position. That is, both Polish and Spanish are placed in the category of "dominant penultimate stress"; while Polish does have obligatory penultimate stress with few exceptions (mostly loanwords), Spanish has a number of words with final and antepenultimate stress (including certain verb tenses with obligatory final stress). Nevertheless, it is reasonable to consider these figures in the context of the

46. Interestingly, a common strategy employed by children who are learning English or Dutch is to truncate initial unstressed syllables, so that all words do start with stressed syllables. Children learning these languages are also sometimes observed to shift stress onto initial weak syllables; see Fikkert (1994), Pater (1997), and references therein. Jusczyk, Houston, \& Newsome (1999) have also shown that infants in an English-language environment are able to segment strong-weak words from running speech at an earlier age than weak-strong words. 
current discussion, since both English and Dutch are themselves languages that show a preference rather than a requirement for initial stress.

In any case, Hyman's (1977) survey indicates that languages that favor stress at or near word-final position - penultimate or final stress-are about as common as languages that favor initial stress. Furthermore, approximately as many languages have phonemic stress as have initial stress. Thus, while it is reasonable to suggest that languages like English and Dutch can facilitate word-segmentation by equating stressed syllables with initial syllables, such a strategy is much less likely to be useful for a language with predominantly penultimate stress, like Polish; predominantly final stress, like Turkish; or lexically contrastive stress, like Russian.

Of course, languages with a tendency to have stress in penultimate or final position may well use the location of the stressed syllable to compute the most likely location for the following word-boundary, in a modified metrical segmentation strategy tailored to the metrical pattern of the language in question. But the crucial point for the present discussion is that actually equating the stressed syllable with the initial syllable is a segmentation strategy that will only be useful for a subset of the world's languages.

This point is made explicitly by proponents of the MSS and similar strategies. Taft (1984) states,

The language-specific version of the strategy specifies which portions of the signal are salient for that language, as well as the relationship between these salient portions and the onsets of words. (Taft 1984:90)

Norris, McQueen, \& Cutler (1995) make a similar statement:

[T]he stress-based metrical segmentation procedure . . . is appropriate for stresstimed languages such as English. Of course, this procedure could not operate for languages in which there is no alternation of strong and weak syllables. Nonetheless, a separate procedure of segmentation based on metrical structure can operate in any language; it is only different across languages insofar as the metrical structures of the languages themselves differ. (Norris, McQueen, \& Cutler 1995:1210)

In conclusion, stressed syllables are used, at least in some languages, to locate word boundaries for the purposes of word recognition. However, unlike initial syllables, stressed syllables are not directly used in early-stage word recognition. Therefore, by the definition introduced above, stressed syllables are legitimately excluded from the set of psycholinguistically strong positions.

Furthermore, since the presence or absence of stress on a given syllable is not relevant in early-stage word recognition (as seen, for example, in Cutler's (1986) 
findings that one word will prime another that differs from it only in stress contour), $\mathbf{M} / \Psi$ str constraints that involve stress, such as HAVESTRESS/Root (§4.2.2.1), are able to pass the Segmental Contrast Condition.

\subsubsection{On tonal contrasts in early-stage word recognition}

In addition to stress, tone is another prosodic property that is used by languages to distinguish lexical items. Because tone can be lexically contrastive, it is logically possible that languages with tonal contrasts might treat tone on a par with segmental features, and disallow $\mathbf{M} / \Psi$ str constraints that make reference to tone. However, it has been shown above that information about stress, although it can be used to form lexical contrasts, is apparently not used in early-stage word recognition. Therefore, it is also possible that tone and stress, both being prosodic properties, might pattern together in this respect. Unfortunately, there have not been as many studies on the extent to which tone is utilized in early-stage word recognition as there have been for stress, and the results that have been found are somewhat mixed.

On the one hand, there is evidence that tonal and segmental contrasts are treated differently by the processor, and in particular that it takes more time for tonal information to become available to listeners than segmental information. If tonal information is indeed slow to become available, it would presumably not be used in early-stage word recognition.

Cutler \& Chen (1997) report that tone takes longer to process than segmental information in Cantonese, a language with several lexical tones. In a "samedifferent" task (which requires deciding whether or not two auditory stimuli represent the same word), participants had slower response times and made more errors when the words in a stimulus pair differed only in tone than when they contained different segments, even in cases where the differing tones were perceptually quite distinct.

Walsh Dickey (in prep.) also found a difference in timing between segmental and tonal information in a same-different task in Igbo, a tone language in which any syllable may bear either a $\mathrm{H}$ or a $\mathrm{L}$ tone. The words used in the experiment were of the shape VCV. In each "different" pair, the words differed in only one feature: a segmental feature in the consonant (C), the first vowel (V1), or the second vowel (V2), or the tone in the first (T1) or second (T2) syllable. Walsh Dickey found that words differing in $\mathrm{Tl}$ had significantly longer response times than words differing in $\mathrm{Vl}$, even though the tonal and segmental mismatches occurred at the same chronological point in the two cases; this finding suggests that segmental information was more readily available than tonal information during the first syllable. On the other hand, words differing in $\mathrm{T} 2$ had response times that were just 
as fast as the V2 cases, suggesting that by the time the second syllable had been reached, tonal and segmental information were both available.

Finally, Cutler \& Chen (1997) found that in a Cantonese lexical decision task, nonwords were more likely to be erroneously labeled as words when they differed from actual words in tone than when they differed in segmental features. These results also seem to indicate that tone is not as salient or useful in word recognition as segmental features are, whether because of a difference in the time at which the information becomes available or for some other reason.

Thus, the results from Cantonese and Igbo seem to show that tonal and segmental features are handled differently in speech perception, and in fact that tonal information becomes available to the processor more slowly than segmental information does. In further support of this finding, acoustic analyses of tone-tosyllable alignment patterns in several languages have shown that tonal targets are often realized somewhat later than the peak of the syllable with which they are phonologically affiliated (see, e.g., Silverman \& Pierrehumbert (1990) on English; Arvaniti, Ladd, \& Mennen (1998) on Modern Greek; and Xu (1999) on Mandarin). If there is a delay in the accessibility of tonal information during speech processing, it may be the case that at the point of early-stage word recognition, differences in lexical tone do not exclude lexical entries from initial activation.

Another language for which the role of tonal features in processing has been investigated is Japanese. Lexical contrasts between syllables bearing $\mathrm{H}$ and $\mathrm{L}$ tone are found in many Japanese dialects, including standard or Tokyo-area Japanese. However, these systems are traditionally classified as pitch-accent systems rather than as tone systems proper, because what must be lexically stored is not a separate tone specification for each syllable, but the location in the word (if any) where the pitch falls from $\mathrm{H}$ to $\mathrm{L}$.

Psycholinguistic evidence for the role of lexical pitch accents in the perception of (Tokyo) Japanese is somewhat mixed. On the one hand, there is evidence, similar to that discussed above for lexical tone in Cantonese and Igbo, that pitch information becomes available to the listener at a later point in time than segmental information. Walsh Dickey (1996) had Japanese-speaking listeners perform a samedifferent task in which some of the "different" pairs contained different segments, but others differed only in accent. Listeners' responses to "different" pairs were much slower when the words differed only in accent than when segmental differences were involved.

However, there is also evidence that pitch accent does have some effect on processing in Japanese. For example, Minematsu \& Hirose (1995) showed that presenting Japanese words with incorrect accent patterns made them harder for experimental participants to identify. 
Perhaps the strongest evidence that Japanese pitch accent is relevant in earlystage word recognition is the finding by Cutler \& Otake (1999) that accent apparently does help to constrain the initial selection of a set of lexical entries to be further compared with the auditory input being processed (unlike stress in English (Cutler 1986); see §4.3.4.2). Words with different tonal shapes, such as ame HL 'rain' and ame LH 'candy,' were found not to behave as homophones in lexicalpriming tasks. This indicates that even in early stages of word recognition, ame $\mathrm{HL}$ is not considered a good match for ame $\mathrm{LH}-$ a decision that would seem to require reference to tonal information.

So it may be that tonal properties, unlike stress, are used in early-stage word recognition, at least for Japanese. The question nevertheless remains as to whether there is a fundamental difference between pitch-accent systems and true tonal systems in this respect, given the results reported above for the tonal languages Cantonese and Igbo where tonal information did not appear to be available in early stages of speech perception. Interestingly, there is some evidence that the relevant difference is not between pitch-accent and true tone systems in general, but rather between Japanese and the other languages investigated specifically.

Walsh Dickey (in prep.) suggests that one reason why tonal information may not be utilized in perception as early as segmental information is that it may be difficult to correctly identify the tone in the first syllable of a word, at least when spoken in isolation. Since tone is relative (i.e., the specific pitches used for "high" and "low" tones will depend on the pitch range of an individual speaker), perhaps the identity of the first tone of an utterance cannot be established without comparing it to subsequent tones. Thus, Walsh Dickey proposes, the tones in the first syllables of the Igbo words cannot be identified until they are compared with the secondsyllable tones. This hypothesis is consistent with the reported reaction times: the time needed for listeners to respond to a difference in first-syllable tone was equivalent to the time needed to respond to a difference in second-syllable tone or vowel quality.

Crucially, however, for Cutler \& Otake's (1999) Japanese listeners, identifying $\mathrm{H}$ versus $\mathrm{L}$ tone probably did not require information from an external context. Cutler and Otake's listeners could tell, with an accuracy significantly above chance, whether a single syllable excised from a word bore $\mathrm{H}$ or $\mathrm{L}$ tone in the word from which it was taken. When they measured the acoustic properties of their experimental materials, Cutler and Otake found that there was a strong correlation between $\mathrm{H}$ tones and level pitch on the one hand, and between $\mathrm{L}$ tones and changing pitch on the other. Furthermore, they found that the tones of the individual excised syllables were most reliably identified by the listeners when they conformed the most strongly to this pattern. Thus, listeners were making use of this kind of 
syllable-internal difference to distinguish $\mathrm{H}$ from $\mathrm{L}$ tones even without a surrounding context to help them identify the speaker's pitch range.

In summary, it can be tentatively concluded that tone is used in early-stage word recognition in some languages but not in others, and that this difference may be related to whether tones can be identified within the syllable to which they are associated, or whether external context is needed. However, there are still open questions: if pitch accent is used in early-stage word recognition in Japanese, why were the same-different results reported by Walsh Dickey (1996) so much slower for accentual differences than for segmental ones? And if being able to identify tones within the context of their own syllable is a crucial factor, why did Cutler \& Chen (1997) find that Cantonese tonal differences required long response times in a same-different task even when the tones were quite different from one another?

At this point, no more will be said about the status of tone in word recognition, especially since the investigation of tone-related positional augmentation constraints is itself a topic for further research (\$4.2.3). It may even turn out to be the case that the decision about whether tone is relevant for early-stage word recognition is determined, not on a universal basis, but with reference to facts about the individual grammar of a language. Nevertheless, there is still a prediction that can be made. Namely, a particular feature is something that can be legitimately referred to by an $\mathbf{M} / \Psi$ str constraint only when that feature is not crucial in early-stage word recognition, and this-not the distinction between "segmental" and "prosodic"-is what is actually encoded in the formulation of the Segmental Contrast Condition (see §2.4.1). The mnemonic label "segmental" used in the name of this filter might need be changed once the relationship between tone and processing is better understood, but the substantive motivation behind the filter remains the same.

\subsubsection{Conclusions: Psycholinguistic evidence and $M / \Psi$ str constraints}

The three preceding sections, $\S \S 4.3 .2-4$, have presented a number of psycholinguistic findings that are relevant for $\mathbf{M} / \Psi$ str constraints. To be sure, a great many of these results come from experiments on Western European languages, especially English and Dutch. It will be interesting to see how future psycholinguistic experimentation on additional, less closely related languages contributes to our understanding of the structures and processes involved in word recognition.

This section now relates the psycholinguistic findings reviewed above to particular aspects of the theory of $\mathbf{M} / \mathbf{s t r}$ constraints laid out in Chapter 2. $\S 4.3 .5 .1$ addresses the exclusion of the stressed syllable from the set of psycholinguistically strong positions and the resulting predictions about possible positional augmentation constraints for stressed syllables. \$4.3.5.2 shows how the 
psycholinguistic evidence presented above is reflected in the formulation of the Segmental Contrast Condition.

\subsubsection{Classifying the stressed syllable}

The psycholinguistic findings discussed above justify the distinction made here between the initial syllable and the root, which are classified as psycholinguistically strong positions, and the stressed syllable, which is not. The initial syllable and the root are especially important in early-stage word recognition-the initial syllable is important because the first few segments of a word have a particularly strong influence over which lexical entries are activated by a given auditory input, and the root is important because roots help determine how morphologically complex words are stored in the lexicon and therefore how they are activated. In contrast, while the stressed syllable may in some languages be used during speech processing to help identify the beginnings of words, it is not directly involved in early-stage word recognition.

The exclusion of the stressed syllable from the set of psycholinguistically strong positions has implications for a general theory of positional augmentation constraints: it predicts that $\mathbf{M} / \sigma^{\sigma}$ constraints should not be subject to any constraint filter that is specifically relevant for $\mathbf{M} / \Psi$ str constraints. Indeed, the Segmental Contrast Condition, an $\mathbf{M} / \Psi$ str constraint filter, does not apply to $\mathbf{M} / \sigma$ constraints. As seen in chart (47) in $\$ 2.3 .3$, there are a number of $\mathbf{M} / \mathbf{\sigma}$ constraints that refer to segmental contrasts (and whose potential $\mathbf{M} / \sigma_{1}$ and/or $\mathbf{M} / \mathbf{R o o t}$ counterparts are blocked by the Segmental Contrast Condition).

\subsubsection{On the formulation of the Segmental Contrast Condition}

The experimental results reviewed in $\$ \S 4.3 .2-4$ identify certain substantive pressures on psycholinguistically strong positions that stem from their special role in early-stage word recognition. The substantive pressures in question are those that have been formalized in the Segmental Contrast Condition (\$2.4.1), repeated here in (77). This section addresses each clause of the constraint filter in turn, showing how it is related to the psycholinguistic evidence. 
If a constraint is of the form $M / \Psi$ str, then it must meet one of the following two conditions:

I. Satisfaction of the $\mathbf{M}$ constraint from which the $\mathbf{M} / \Psi$ str constraint is built does not alter features that are distinguished in early-stage word recognition.

or

II. $\Psi$ str is $\sigma_{1}$, and satisfaction of the $M / \Psi$ str constraint serves to demarcate the left edge of $\sigma_{1}$.

Clause I of the Segmental Contrast Condition reflects two aspects of early-stage word recognition that have been identified in $\$ \S 4.3 .2-4$ : in this phase of word recognition, (a) psycholinguistically strong positions are influential in determining which lexical entries are to be activated, and (b) segmental properties are more important than prosodic properties such as stress in determining how well a given lexical entry matches the incoming auditory signal. Clause I follows proposals by, e.g., Nooteboom (1981) and L. Taft (1984) that speech processing is more efficient if psycholinguistically strong positions are given a large number of phonological contrasts to draw from, since these positions have a particularly strong influence on determining which lexical entries are activated in early-stage word recognition. Behind this claim is the assumption that using a larger number of contrastive categories divides the lexicon more finely, so that there will be fewer lexical entries matching any given initial sequence (this assumption is examined more closely below). However, clause I differs from its antecedents in the literature by specifically excluding prosodic properties such as stress (\$4.3.4.1) and perhaps tone (\$4.3.4.2) from its scope. Because prosodic properties are effectively disregarded at this stage of word recognition, whether or not contrasts involving these properties are available in psycholinguistically strong positions would have no particular influence on word recognition.

The idea that contrast preservation in psycholinguistically strong positions is important for the efficiency of word recognition has been discussed by, among others, Nooteboom (1981), L. Taft (1984), Beckman (1997, 1998), and Casali $(1996,1997)$. Nooteboom (1981) speculates about the implications that a special role for initial material in processing might have for the distribution of phonological information within words. Nooteboom points out that the beginning of the word is particularly informative, both because it is the least predictable (i.e., in most cases, 
no previous phonological information about the content of the word in question is available to the hearer), and also because, as discussed above, it has been demonstrated to carry more weight in early-stage word recognition. Nooteboom suggests that languages are likely to have made use of the asymmetry in informational content between initial and later material.

In phonological terms one would predict that (1) in the initial position there will be a greater variety of different phonemes and phoneme combinations than in word final position, and (2) word initial phonemes will suffer less than word final phonemes from assimilation and coarticulation rules. (Nooteboom 1981:422)

Taft (1984:229) makes a similar observation, stating that crosslinguistically, "processes which cause phonological contrasts to be lost should be permitted wordfinally but not word-initially." Taft notes that if word-initial material is important in early-stage word recognition, then a reduction in the number of word-initial contrasts by some phonological process would result in more lexical entries matching every possible initial syllable, and this might lead to a greater computational load.

These considerations predict that positional augmentation constraints for psycholinguistically strong positions are to be dispreferred. ${ }^{47}$ Positional augmentation constraints require the presence of properties that bolster the perceptual prominence of strong positions. In general, such constraints are compatible with the intrinsic prominence that strong positions have; in other words, positional augmentation constraints make strong positions stronger by giving them an additional perceptually salient characteristic (\$2.3.1). However, if a positional augmentation constraint is satisfied, this does mean that a phonological contrast in a strong position is neutralized. Thus, the substantive pressure for psycholinguistically strong positions to preserve contrasts relevant for early-stage word recognition means that (segmental property) neutralization is to be avoided-even when that neutralization would enhance perceptual prominence, as in the case of positional augmentation.

As noted above, the claim that contrast neutralization in psycholinguistically strong positions is something to be avoided depends on a particular assumption: that

47. Another linguistic consequence of the pressure to maintain a larger number of contrasts in psycholinguistically strong positions is the very inclusion of these positions in the set of strong positions, which gives them positional faithfulness constraints- $-F / \sigma_{1}$ constraints and $\mathbf{F} / \mathbf{R o o t}$ constraints - and the corresponding potential for resisting positional neutralization (see Beckman 1995, 1997, 1998 and Casali 1996, 1997; positional faithfulness and other analytical approaches to positional neutralization are further considered in §5.2). 
having more contrasts in positions important for early-stage word recognition facilitates word recognition. This assumption is clearly supported by models of word recognition that were influential when Nooteboom and Taft wrote the statements just quoted. For example, in the Search model (Forster 1976, 1990), the first few segments in the auditory signal are used to select an access code, which is linked to all lexical entries that begin with those segments. The early version of the Cohort model (Marslen-Wilson \& Welsh 1978; Marslen-Wilson 1984) does not directly incorporate access codes, but analogously, the first few segments perceived are used to select a "cohort" of all lexical entries that begin with those segments. These models make a straightforward prediction that a language with more segmental contrasts available in the psycholinguistically strong positions will be more efficient at finding the lexical entry that matches an incoming signal. Having more segments to start words with means that fewer words need begin with the same sequence of segments. As a consequence, the set of lexical entries activated in early-stage word recognition by any given initial segment sequence will be on average smaller, which makes the task of narrowing the set down to one item computationally simpler.

However, more recent models of word recognition do not determine the initially activated set of lexical entries in the same way as the Search and early Cohort models. The assumption that word recognition is facilitated by having more contrasts in psycholinguistically strong positions therefore needs to be reexamined.

A major problem with the Search and early Cohort models is that their implementation of the special role of initial material in word recognition is too inflexible. In particular, the set of lexical entries activated in early-stage word recognition cannot be constrained by categorical decisions about the identity of the initial few segments, because if one of those segments happened to be misperceived, it would be impossible for the processor to recover-even if the misperception involved only one feature of one of the initial few segments, the target word would not be present in the set of lexical entries activated under the selected access code or in the selected cohort. In fact, changing early segments in a word does hamper recognition more than changing later segments, but it does not necessarily prevent recognition, or initial activation, altogether (see $\S 4.3 .2$ ).

Subsequent two-stage models of word recognition, such as the later version of Cohort (Marslen-Wilson 1987), Shortlist (Norris 1994; Norris, McQueen, \& Cutler 1995), and the Neighborhood Activation Model (Luce 1986; Goldinger, Luce, \& Pisoni 1989; Luce \& Pisoni 1998), have addressed this problem by having the auditory input activate a set of lexical entries that are sufficiently similar to, though not necessarily a complete match for, the incoming auditory signal. Thus, the target word will no longer be incorrectly excluded from the initially activated set if one of its first few segments is misperceived, because words with segments that are 
sufficiently similar to the perceived segments will all receive some activation. (The special role of word-initial material can still be implemented in such a model by giving extra activation to lexical entries whose initial portions are a good match to the incoming signal, as suggested by Marslen-Wilson (1987).)

In these later two-stage models of word recognition, where sufficient similarity determines which lexical entries are to be activated, it is of course necessary to specify how similarity is to be measured. The model that is most explicit about how to characterize similarity among lexical items is the Neighborhood Activation Model, henceforth NAM (Luce 1986; Goldinger, Luce, \& Pisoni 1989; Luce \& Pisoni 1998). In the NAM, as in other two-stage models, the acoustic/phonetic input activates a set of lexical entries that will subsequently be narrowed down to the best match. What determines the members of the activated set is a distance measure involving their degree of acoustic/phonetic similarity to the incoming signal.

In the NAM, the neighborhood of a target word is the acoustic/phonetic space centered on that word such that other words in the neighborhood-that is, neighbors-will receive (a significant degree of) lexical activation when the target word is presented. (So far, however, only a rough characterization of this acoustic/phonetic space has been given; see below for further discussion.) Because neighbors are activated together and must compete with each other, the model predicts that words in dense neighborhoods, with many neighbors, are more difficult to recognize than words in sparse neighborhoods, with few neighbors.

Experimental findings by Luce, Pisoni, and their colleagues support the NAM as a model of activation and competition in word recognition: words in sparse neighborhoods are indeed easier to recognize than words in dense neighborhoods. This result suggests that sufficiently similar in lexical activation must be defined in terms of acoustic/phonetic distance, not by some relative measure like "the $n$ most similar lexical entries to the incoming signal." Otherwise, the density of words within a neighborhood as defined by acoustic/phonetic distance would not be relevant.

The crucial question that must be addressed for clause I of the Segmental Contrast Condition is this. Does having a larger number of contrastive segments in psycholinguistically strong positions still facilitate word recognition under a model of processing like the NAM, as it does under an older model like Search or early Cohort? Or is having a larger number of contrastive segments now predicted to inhibit word recognition because the inventory would contain more phonemes that are phonetically similar, potentially leading to denser neighborhoods?

The answer to this question is not yet completely clear, because it depends on exactly how the similarity neighborhood for a given word is to be defined. Goldinger, Luce, \& Pisoni (1989) and Luce \& Pisoni (1998) discuss experiments that were carried out on three-phoneme words, mostly of the shape CVC. Given the 
constrained nature of their experimental corpus, they were able to use a simple definition of neighbor: any word that can be converted to the stimulus word by the addition, deletion, or substitution of a single phoneme. Under this working definition of similarity neighborhood, one would in fact be forced to conclude that a language with a larger inventory of contrastive elements will have denser neighborhoods than a language with a smaller inventory. That is, more distinct CVC combinations are possible where there are more Cs and Vs in the inventory, so a given CVC word would be expected to have more neighbors when the language has more phonemes.

However, it is highly doubtful that this simple algorithm reflects the way that neighborhoods are actually determined. As Luce and Pisoni observe,

Clearly, this method of computing neighborhood membership makes certain strong assumptions regarding phonetic similarity. In particular, it assumes that all phonemes are equally similar and that the similarities of phonemes at a given position are equivalent (Luce \& Pisoni 1998:16).

Furthermore, and perhaps more importantly, the above method of identifying neighbors will exclude any word that contains the stimulus word but is longer than the stimulus word by more than one phoneme. This situation is not considered by Goldinger, Luce, \& Pisoni (1989) or Luce \& Pisoni (1998) because their experiments are restricted to three-phoneme words. However, many researchers, including Shillcock (1990) and Norris, McQueen, \& Cutler (1995), have found that the presentation of a short word, such as can, also activates longer words that contain it, such as candle or cantaloupe. If these longer words are activated, then the model must be modified to treat them as neighbors.

When the problem of words embedded inside longer words is considered, the picture changes drastically. Given that speech processing happens across time, it is highly likely that words whose first few segments are identical-even if one word is much longer than the other-are much stronger competitors, which is to say treated during early-stage word recognition as much closer neighbors, than words beginning with phonetically similar but nevertheless distinct phonemes (see the Shillcock (1990) and Norris, McQueen, \& Cutler (1995) findings for embedded words just mentioned, and Zwitserlood's (1989) findings for initial partial primes discussed in $\S 4.3 .2 .2$ above). Crucially, a language with a larger phoneme inventory can populate its lexicon with more distinct short words than a language with a smaller inventory can; having more short words means having fewer longer words that contain other words of the language.

Thus, even in more recent models of word recognition that define the initially activated set of lexical entries, not in terms of a categorical match to the initial few 
segments of the input, but in terms of acoustic/phonetic distance from the perceived input, it still appears that having a larger number of contrasts facilitates word recognition. Thus, there is a genuine substantive pressure behind clause I of the Segmental Contrast Condition.

One further point to note is that the substantive pressure for psycholinguistically strong positions not to neutralize segmental contrasts, as formalized in clause I, does not mean that contrasts are never neutralized in those positions; clearly, this is not the case. For example, a general markedness constraint banning some typologically marked segmental property is often ranked above all faithfulness constraints in a given language (including any positional faithfulness constraints), so that the segmental property in question is never contrastive in any position, including psycholinguistically strong positions. After all, large segmental inventories, as helpful as they may be for partitioning the lexicon, are problematic for other substantive reasons; for example, large inventories must include segments that are less perceptually distinct from one another and involve greater articulatory complexity. The point is simply that neutralizing some contrast specifically in a psycholinguistically strong position, where it would have been helpful in word recognition, while still putting forth the perceptual and articulatory effort to maintain that contrast in other positions, is not an efficient allocation of resources. It is this particular situation that satisfaction of a segmental-contrast $M / \Psi$ str constraint would lead to, and that is why there is a filter that eliminates precisely such constraints.

In summary, clause I of the Segmental Contrast Condition reflects a substantive pressure that is based on two facts about early-stage word recognition: psycholinguistically strong positions are important at that stage, and certain contrasts are much more influential than others in distinguishing lexical entries during that stage. Thus, clause I allows a given $\mathbf{M} / \Psi$ str constraint to be included in CON only if its satisfaction would not entail the neutralization of contrasts that are used to distinguish lexical entries during early-stage word recognition.

The substantive factors behind clause II of the Segmental Contrast Condition are somewhat less complex. First, segmentation of the speech stream-finding the edges of words-is a nontrivial aspect of auditory processing (§4.3.4.1.3). Furthermore, having an onset and having a lower-sonority onset are both ways of making a syllable more perceptually prominent $(\$ 2.3 .2 .3 .1)$. As noted by Taft (1984),

[A] word recognition model which makes crucial use of word onsets ... would value a phonological system which marks word onsets as clearly as possible. Thus, if a language permits phonological processes word-initially, they should be processes which make the [word] onset more salient. (Taft 1984:229) 
Thus, clause II of the Segmental Contrast Condition permits a $\mathbf{M} / \Psi$ str constraint to be included in CON if $\Psi_{s t r}$ is $\sigma_{1}$ and satisfaction of the constraint affects the leftmost segment (see §2.4.1). Since these two clauses form a disjunction, an $\mathbf{M} / \Psi$ str constraint is legitimate if it passes at least one of the two. As a consequence, ONSET $/ \sigma_{1}$ and $\left[{ }^{*} \mathrm{ONSET} / \mathrm{X}\right] / \sigma_{1}$ are legitimate constraints, because even though they refer to segmental properties in a psycholinguistically strong position, failing clause I, they aid in left-edge demarcation, thereby passing clause II.

In conclusion, the Segmental Contrast Condition, like the Prominence Condition, is a constraint filter that models substantive requirements on positional augmentation constraints. Unlike the Prominence Condition, however, the Segmental Contrast Condition applies specifically to positional augmentation constraints for psycholinguistically strong positions. The substantive requirements involved in the Segmental Contrast Condition are all related to early-stage word recognition, which is to be expected, given that psycholinguistically strong positions are precisely those positions that are important in that particular aspect of speech processing.

\subsection{Defining "initial syllable"}

This chapter has examined a number of topics related to positional augmentation in psycholinguistically strong positions. The discussion began with examples of languages in which $\mathbf{M} / \Psi$ str constraints play an active role in phonological patterning. Then, psycholinguistic evidence related to these positions and their role in early-stage word recognition was considered in some detail to justify the particular implementation of the Segmental Contrast Condition that was proposed in $\$ 2.4 .1$.

There is one more question related to positional augmentation in psycholinguistically strong positions that needs to be considered: what kind of "initial syllable" is actually a strong position? This section shows that the patterning of $\mathbf{M} / \sigma_{1}$ constraints within the broader theory of positional augmentation supports the choice of initial syllable of the morphological word (MWd) as the designation of the strong position in question (\$4.4.1). A comparison of this result with Beckman's $(1995,1997,1998)$ choice of root-initial syllable as the position that is relevant for $F / \sigma_{1}$ constraints is then given $(\$ 4.4 .2)$. 


\subsection{1 "Initial syllable" as MWd-initial syllable}

The languages discussed in $\S 4.2 .1$ provide evidence for the existence of $\mathbf{M} / \sigma_{1}$ constraints, specifically, for ONSET/ $\sigma_{1}$ and members of the $\left[{ }^{*} \mathrm{ONSET} / \mathrm{X}\right] / \sigma_{1}$ subhierarchy. Arapaho and Guhang Ifugao are languages in which "initial syllables" are required to have onsets; Mongolian, Kuman, Guugu Yimidhirr, Pitta-Pitta, Mbabaram, and the Sestu dialect of Campidanian Sardinian are languages in which high-sonority elements are banned from onset position in "initial syllables." This section shows that for these languages, the onset-related requirements hold of wordsized, not root-sized, domains ( $\$ 4.4 .1 .1)$, and argues that the "word" in question must be the morphological word rather than the prosodic word $(\operatorname{PrWd})(\S 4.4 .1 .2)$.

\subsubsection{Evidence for a word-sized domain}

Of the languages from $\$ 4.2 .1$, several—Mongolian, Kuman, Guugu Yimidhirr, Pitta-Pitta, and Mbabaram-have no prefixes, which means that roots are always word-initial. These languages cannot help determine whether the requirements on "initial syllables" are actually enforced on word-initial or root-initial syllables. However, Arapaho and Guhang Ifugao (as well as Sestu Campidanian, discussed in $\$ 4.4 .1 .2$ below) provide crucial evidence that it is a word-sized constituent that is relevant.

Arapaho (Salzmann 1956; $\$ 4.2 .1 .1$ ) is a language in which ONSET $/ \sigma_{1}$ ensures that all "initial syllables" have onsets. According to Salzmann (1956:55, note 9), Arapaho has prefixes. Nevertheless, Salzmann clearly states,

There are four vowel phonemes in Arapaho... They are found only medially and finally, thus contrasting with consonants which are found also initially. (Salzmann 1956:51; emphasis added)

Since there are words that begin with prefixes-that is, not all words begin with roots-an onset requirement for root-initial syllables cannot account for mandatory word-initial onsets in Arapaho. The relevant "initial syllable" must be a word-initial syllable.

Similarly, Guhang Ifugao (Newell 1956; Landman 1999) is a language with mandatory onsets in "initial syllables" enforced by ONSET $/ \sigma_{1}$. Like Arapaho, it has prefixes, but all words, including those that do not start with roots, must have an onset in the initial syllable. 
Syllable patterns VC and V may not occur word-initially. All words begin with consonants. (Newell 1956:523; emphasis added)

Thus, the relevant "initial syllable" for Guhang Ifugao is also a word-initial syllable; enforcing onsets only in the initial syllables of roots would again be inadequate.

However, the question arises whether an onset requirement for root-initial syllables might be necessary in Guhang Ifugao in addition to one for word-initial syllables (see also the discussion of root-initial faithfulness effects in $\S 4.4 .2$ below). Landman (1999) observes that there appear to be constituents smaller than the word that are forced to have onsets. For example, the prefix /mun-/ attaches only to forms with initial onsets (78).

Initial onset requirement inside complex words (Landman 1999:15)

$\begin{array}{llll}\text { mun.?u.gút } & \text { 'sew' } & \text { mun.há.pit } & \text { 'speak' } \\ \text { mun.?ag.gé.a? } & \text { 'I'm looking for' } & \text { mum.bú.un } & \text { 'wearnecklace' } \\ \text { mun.?o.lé } & \text { 'do slowly' } & \text { mun.lá.ik } & \text { 'squash' } \\ \text { mun.?út.?ut } & \text { 'pain' } & \text { mun.na.nón } & \text { 'remain' }\end{array}$

The potential problem is this. Roots are always consonant-initial when they occur word initially (i.e., without /mun-/), because in that context high-ranking ONSET $/ \sigma_{1}$ is relevant. By the principle of richness of the base, even a potential input consisting of a vowel-initial root will surface unfaithfully, so that it starts with a consonant (Landman 1999 suggests that [?] has been epenthesized in such cases; see $\S 4.2 .1 .1$ ). However, ONSET $/ \sigma_{1}$ (where $\sigma_{1}$ is word-initial syllable) is unable to compel a root to surface consonant-initially when it is preceded by /mun-/. In other words, what prevents a vowel-initial root like the hypothetical /ole/ from surfacing as [?ole] in isolation, but as *[mu.no.le] when prefixed by /mun-/?

It turns out that even positing an ONSET constraint for root-initial syllables is not helpful here. A putative ONSET/ $\sigma_{1-\mathrm{Rt}}$ constraint, requiring root-initial syllables to begin with onsets, would not distinguish between [mun.?o.le] and a form like *[mu.no.le], in which the prefix-final consonant $/ \mathrm{n} /$ is syllabified to provide the root-initial syllable with an onset: both candidates would satisfy the constraint.

Fortunately, there are a number of promising solutions to the problem of *[mu.no.le]-type forms, which do not require positing an ONSET $/ \sigma_{1-R t}$ constraint in addition to the ONSET $/ \sigma_{1}$ already needed for word-initial syllables in this and other languages. For example, output-output faithfulness constraints could be invoked to ensure that roots embedded in complex words have the same form that 
they take in isolation-namely, with an initial consonant. ${ }^{48}$ Alternatively, it could be the case that /mun-/ subcategorizes for a word rather than for a bare root, so that words with /mun-/ have the structure $\left[_{\mathrm{MWd}}\right.$ mun $\left[{ }_{\mathrm{MWd}}\right.$ ?ole ]], and ONSET/ $\sigma_{1}$ itself is relevant for the initial syllable of the embedded MWd as well as the initial syllable of the complex form. ${ }^{49}$

Thus, for Arapaho and Guhang Ifugao, the requirement for "initial" onsets clearly holds of word-sized domains. Conversely, there is no conclusive evidence that a similar requirement holds specifically of roots. "Initial syllable" is therefore best characterized as "word-initial syllable."

\subsubsection{Defining the relevant word-sized domain}

The previous section has argued that augmentation phenomena affecting the "initial syllable" show that the relevant syllable is one that is initial in a word-sized domain. Two kinds of word-sized constituents are relevant in phonology: the morphological constituent MWd and the prosodic constituent PrWd. However, the theory of positional augmentation constraints developed here is most compatible with $M W d$ initial syllable as the characterization of the strong position in question, because of the status of the initial syllable as a psycholinguistically strong position.

As discussed above and in Chapter 2, there are two main sources of evidence that the initial syllable is a strong position for psycholinguistic reasons. First, experimental results show that initial material is important in early-stage word recognition (\$4.3.2). Second, attested initial-syllable augmentation constraints are more limited than those for the phonetically strong position stressed syllable, a

48. For other examples of languages in which requirements that hold of roots in isolation are extended through high-ranking output-output faithfulness constraints to hold of roots inside complex forms as well, see the discussion of "paradigm occultation" in McCarthy (1998).

49. This second approach has some similarities to Cohn \& McCarthy's (1998) analysis of a similar pattern in Indonesian. Cohn \& McCarthy invoke an alignment constraint that requires left-edge alignment between a root and a PrWd. This forces a syllabification like məy. [PrWd 2 i.si] 'fill' from /məN $+[\text { isi }]_{\mathrm{Rt}}$, because syllables cannot cross PrWd boundaries. However, one difference between Indonesian (or at least in the dialect of Indonesian described by Cohn $(1989,1993)$ and Cohn \& McCarthy (1998)) and Guhang Ifugao is that words without initial onsets are tolerated in Indonesian. The two approaches to Guhang Ifugao suggested above have the advantage of relating the inability of final consonants in prefixes like /mun-/ to be syllabified into the onset position of a hypothetical V-initial root to the requirement that roots have initial onsets in isolation. 
position of equivalent size (see (47) in $\$ 2.3 .3$ and (70) in $§ 4.2 .3$ ). If the initial syllable were a phonetically strong position like the stressed syllable, the two positions would be expected to have the same set of positional augmentation constraints.

Given that the initial syllable is a psycholinguistically strong position, it must be the MWd, not the PrWd, that is the relevant word-sized domain. Psycholinguistic evidence shows that morphological information is relevant in early-stage word recognition and lexical organization ( $\$ 4.3 .3)$, but prosodic information bears only an indirect relationship to early-stage word recognition (\$4.3.4). Since psycholinguistically strong positions are defined as those positions that are critically important in early-stage word recognition-a definition that is further supported by the different behavior of initial syllables and stressed syllables with respect to their inventories of positional augmentation constraints-a morphologically defined word is the more theoretically appropriate choice.

The remainder of this section considers and rejects potential evidence that the PrWd is the relevant domain for positional augmentation in the "initial syllable" after all. First, an apparently phrasal metathesis pattern in the Sestu dialect of Campidanian Sardinian (see also §4.2.1.2.1), presented by Bolognesi (1998) as evidence that rhotics are banned specifically in PrWd-initial position, is argued not to distinguish between MWd and PrWd; that is, while there is no evidence from Sestu to prove that MWd is the domain in question, Sestu is shown at least to be compatible with the MWd proposal. Then, the tendency toward initial-consonant fortition in large prosodic domains, which resembles some of the initial-syllable onset effects seen in $\S 4.2 .1$, is addressed. Prosodic domain-initial fortition is shown to be a phonetic rather than a phonological effect. This means that it cannot be directly related to true initial-syllable augmentation effects, which are phonological. Thus, the existence of initial fortition in large prosodic domains is not of itself evidence that the domain of the phonologically relevant "initial syllable" must be prosodically defined.

In Sestu Campidanian (Bolognesi 1998), as discussed in §4.2.1.2.1, rhotic (and glide) onsets are prohibited in "initial syllables." Contrary to the proposal made here, Bolognesi (1998:Ch 8) argues, based on a rhotic metathesis phenomenon, that it is specifically the PrWd whose initial syllable may not have a rhotic onset. However, the data that Bolognesi presents to support his claim do not, in fact, conclusively distinguish between PrWd and MWd.

Bolognesi shows that, in Sestu and other Campidanian dialects of Sardinian, there are a number of metathesis phenomena-both synchronic and 
diachronic-involving $[\mathrm{r}] .^{50}$ First, there are a number of Sestu words of the shape $\mathrm{CrVC:V}$ that correspond to Standard Sardinian words of the shape $\mathrm{CVrCV}$, suggesting that there has been (diachronic?) metathesis creating a complex onset in order to improve performance on NoCODA. ${ }^{51}$

(79) [r] coda-to-complex onset metathesis (Bolognesi 1998:419)

$\begin{array}{lll}\begin{array}{l}\text { Sestu } \\ \text { prok:u }\end{array} & \text { Standard Sardinian } & \\ \text { tsrup:u } & \text { porku } & \text { 'pig' } \\ \text { fratf:i } & \text { tsurpu } & \text { 'blind' } \\ \text { krup:a } & \text { fart } 5 i & \text { 'sickle' } \\ \text { mrats:u } & \text { marpa } & \text { 'fault' } \\ \text { kruts:u } & \text { kurts:u } & \text { 'rotten (of cheese)' } \\ \text { sroyu } & \text { sorgu } & \text { 'short' } \\ \text { sre'ßi(ri) } & \text { ser'bi(ri) } & \text { 'father in law' } \\ \text { sprazi } & \text { spardzi } & \text { 'to be useful' } \\ \text { mrazini } & \text { mardzini } & \text { 'to spread' } \\ & & \text { 'margin' }\end{array}$

The coda-[r] metathesis shown in (79) is pervasive. In fact, Bolognesi (1998:419) states, "In Sestu Campidanian and other related dialects, the liquid $/ r /$ is found in coda position only in a limited set of words, all of which exhibit the same prosodic structure." Namely, the words that maintain coda [r] are disyllabic and vowel-initial (80).

50. In addition to the two [r]-metathesis phenomena discussed here, there are others not directly related to initial-onset sonority effects, such as the migration of an [r] from a complex onset in a final syllable into a complex onset in the preceding syllable. See Bolognesi (1998) for discussion.

51. The gemination of the medial voiceless stops (phonetically realized not as length but as resistance to certain lenition processes) and the lenition of the medial voiced stops in the metathesized forms in (79) are the usual realizations of "long" consonants in Sestu; that is, the base prosodic position of the [r] seems to be maintained in the metathesized form, triggering compensatory "lengthening." See Bolognesi (1998) for further discussion of "long" segments and their phonetic realizations.

52. Bolognesi (1998:419) actually lists these two forms in the opposite columns, but they are listed correctly on p 35 (and the Sestu form is given as sro pu several times in the text). 
(80) [r] codas maintained (Bolognesi 1998:419)

$\begin{array}{llll}\text { orku } & \text { 'ogre' } & \text { ardza } & \text { 'tarantula' } \\ \text { arku } & \text { 'bow' } & \text { argu } & \text { 'sour' } \\ \text { عrba } & \text { 'grass' } & \text { ordzu } & \text { 'barley' }\end{array}$

It is clear (as Bolognesi also notes) that the absence of [r] metathesis in these contexts is related to the prohibition of word-initial rhotic onsets in Sestu. If the coda $[r]$ were to move into the onset of its syllable, as in (79), it would become the leftmost onset consonant in the initial syllable: *rok.u. (Metathesis in the opposite direction, producing forms such as *okru, would violate a general prohibition against $\mathrm{Cr}$ onset clusters in word-final syllables; see Bolognesi (1998:Ch 8) for discussion.)

Interestingly, these vowel-initial, [r]-coda words productively undergo metathesis when they are preceded by a determiner (81). It is this fact that leads Bolognesi to conclude that the ban on rhotic onsets in Sestu must be sensitive to initial position in the PrWd; once there is prosodically dependent material preceding the morpheme in question, $[r]$ does appear in initial position within the morpheme, because now it can do so without being initial in the PrWd.

Rhotic metathesis (Bolognesi 1998:419)

(a) Isolation form

$\begin{array}{ll}\text { orku } & \text { 'ogre' } \\ \text { arku } & \text { 'bow' } \\ \text { عrba } & \text { 'grass' } \\ \text { ard3a } & \text { 'tarantula' } \\ \text { argu } & \text { 'sour' } \\ \text { ord3u } & \text { 'barley' }\end{array}$

(b) With determiner preceding

$\begin{array}{ll}\text { s: rok:u } & \text { 'the ogre' } \\ \text { kust rak:u } & \text { 'this bow' } \\ \text { kus: reßa } & \text { 'that grass' } \\ \text { s: raza } & \text { 'the tarantula' } \\ \text { s: rayu } & \text { 'the sour one' } \\ \text { s: rozu } & \text { 'the barley' }\end{array}$

S: rok:u 'the ogre'

kust rak:u 'this bow'

kus. reßa 'that grass'

s. raza

s: rozu 'the barley'

However, there are a number of complications that weaken Bolognesi's claim that it is crucially PrWd-initial position where rhotic onsets are prohibited. First, no supporting evidence is presented to confirm that these determiners are functioning as clitics (that is, morphemes that are prosodically integrated but still morphosyntactically separate). If it turns out that no such evidence is available, it might be the case that these determiners have become prefixes, actually combining with nouns morphosyntactically to form a complex MWd, so that a metathesized [r] as in (81b) is no longer in MWd-initial position (and therefore does not violate 
*[ONSET/RHO] $/ \sigma_{1}$ ). Note that there is more going on here than metathesis: the determiners appear in their pre-vocalic forms (compare the pre-consonantal forms $s a / s: a, k u s t u / k u s t a, k u s a / k u s \cdot a)$ even though the nouns, having undergone metathesis, are not actually vowel-initial. This opaque interaction may be an indication of the morphologization of these determiner+noun combinations. ${ }^{53}$

Thus, pending further investigation, it can be concluded that the Sestu metathesis facts as discussed by Bolognesi (1998) do not really favor PrWd over MWd as the appropriate characterization of the domain in which the "initial syllable" is a strong position.

A second phenomenon that might appear to support the choice of PrWd-initial syllable as the proper characterization of this strong position is the occurrence of initial-syllable onset fortition at various levels of the prosodic hierarchy. If it is a general characteristic of every prosodic level that consonants in domain-initial position tend to undergo fortition, then one might propose that ONSET $/ \sigma_{1}$ and ${ }^{*}[\mathrm{ONSET} / \mathrm{X}] / \sigma_{1}$ are simply the instantiation of this phenomenon at the PrWd level. However, there are two reasons why these $\mathbf{M} / \sigma_{1}$ constraints should not be equated with prosodic domain-initial effects: first, initial-onset fortition in phrasal and larger prosodic categories is phonetic (gradient and variable), not phonological (categorical); and second, initial syllables of large prosodic domains are never seen to license a larger array of phonological contrasts than other positions, which is an important diagnostic of strong-position status (Beckman 1997, 1998). ${ }^{54}$ Thus, being initial in a prosodic domain-including PrWd-does not give a syllable the status of a phonologically relevant strong position, able to resist positional neutralization and to undergo phonological, categorical positional augmentation. It is therefore the initial syllable of a MWd, not the initial syllable of a PrWd, that is a member of the set of strong positions.

Prosodic domain-initial fortition has been documented for a number of phonetic properties in several languages (e.g., Pierrehumbert \& Talkin 1992; Silva 1992; Jun 1993; Dilley, Shattuck-Hufnagel, \& Ostendorf 1996; Fougeron \& Keating 1997; Fougeron 1998; Keating, Cho, Fougeron, \& Hsu 1998; Lavoie 2000;

53. Moreover, recall from $\$ 2.3 .3$ that, since relationships between morphological and prosodic categories must be mediated by segments, the MWd-initial syllable has been defined as the leftmost syllable whose head is affiliated with a given MWd. Under this definition, even if the determiners and nouns in (81) do not combine morphosyntactically to form a complex MWd, the $[\mathrm{r}]$ in the metathesized forms (81b) still does not violate *[ONSET $/$ RHO $] / \sigma_{1}$, because (by virtue of resyllabification of the determiner-final $\mathrm{C}$ ) the $[\mathrm{r}]$ is not the leftmost onset segment in the MWd-initial syllable.

54. On the other hand, there is evidence that heads of the various prosodic constituents do have special status with respect to faithfulness. See §1.3.1, footnote 7 . 
Cho \& Keating 2001; Keating, Wright, \& Zhang 2001). For example, Keating, Cho, Fougeron, \& Hsu (1998) found that speakers of French, Korean, and Taiwanese all made some distinction between smaller and larger prosodic categories in terms of the stop closure duration and/or degree of linguopalatal contact in domain-initial $[t]$ and/or [n], although the specific levels of the prosodic hierarchy that were distinguished varied by language, by speaker, and by consonant. The VOT of voiceless aspirated stops is longer in phrase-initial than in word-initial position in English (Pierrehumbert \& Talkin 1992) and Korean (Jun 1993). Domain-initial [n] has less nasalization (as measured by low-frequency spectral components) in French (Fougeron 1998) and Korean (Cho \& Keating 2001) in higher-level prosodic domains.

Thus, there is a fair amount of evidence that consonant articulations are more extreme or prototypically consonantlike (that is, "stronger") in the initial position of a given prosodic domain than in medial positions within the domain; e.g., a word-initial consonant that is also in phrase-initial position is often stronger than a word-initial consonant in phrase-medial position, and a phrase-initial consonant that is also in utterance-initial position is often stronger than a phrase-initial consonant that is internal to an utterance. Furthermore, in many cases, the effect is cumulative, so that progressively stronger articulations are seen at the edges of progressively larger prosodic constituents. One striking example is VOT in Korean [ $t$ ], for which three different speakers all had increasingly longer VOT in the initial position of prosodic words, accentual phrases, intonational phrases, and utterances (Keating et al. 1998; Cho \& Keating 2001).

However, these domain-initial fortition effects are typically gradient and variable; the Korean VOT results just described notwithstanding, it is more common to find that different speakers distinguish different levels of the prosodic hierarchy to different degrees for different consonants. Cho \& Keating (2001) state, "[W]e do not assume that the effects of prosody on articulation are strictly categorical ... Indeed, in our previous work we have never found uniformity across speakers of a language as to how many or which prosodic constituents show initial strengthening." In this important respect, prosodic domain-initial fortition differs from initial-syllable onset epenthesis and onset sonority ${ }^{55}$ effects, which are phonological phenomena resulting from the satisfaction of ONSET $/ \sigma_{1}$ and *[ONSET $/ \mathrm{X}] / \sigma_{1}$ constraints.

55. In fact, according to Cho \& Keating (2001), Fougeron (1998) argues that the decrease in nasalization in French [ $\mathrm{n}$ ] in the initial position of larger prosodic domains is best analyzed as the result of muscular tension in the levator palatini, rather than as the result of some general trend toward lower sonority in domain-initial positions. 
All this is not to say that prosodic domain-initial fortition is completely unrelated to $\mathbf{M} / \sigma_{1}$ constraints. For example, the general tendency toward domaininitial fortition may bolster the substantive pressure formalized as clause II of the Segmental Contrast Condition (which allows segmental-contrast $\mathbf{M} / \Psi$ str constraints if they are related to left-edge demarcation; $\S 2.4 .1, \S 4.3 .5$ ), on the grounds that MWd-initial and PrWd-initial positions often overlap. However, it is not PrWd status alone that has the ability to make a syllable a phonologically relevant strong position.

In conclusion, evidence from the locus of "initial-syllable" augmentation phenomena in morphologically complex words, and considerations stemming from the psycholinguistic basis of the strong-position status of the "initial syllable," indicate that it is the MWd-initial syllable that is a member of the class of strong positions recognized by the phonology and available for constructing positional faithfulness and augmentation constraints.

\subsection{2 "Initial syllable" and resistance to positional neutralization}

In the Schema/Filter model of CoN (\$2.2.1), constraints are constructed from a set of general constraint schemas that freely apply to elements from a set of phonological primitives and thereby generate individual, contentful constraints. As discussed in $\$ 2.2 .1 .1$, the set of strong positions str is included in the set of phonological primitives, and elements from the set str are selected by the C/str schema, which relativizes constraints, including both markedness and faithfulness constraints, to strong positions. Thus, the positions in the set str are the only formally possible positions to which otherwise context-free constraints can be relativized. ${ }^{56}$ Crucially, both positional faithfulness and positional augmentation constraints make reference to the same set of strong positions. Therefore, it is important to consider whether the conclusions concerning the designation of "initial syllable" as MWd-initial syllable, which have been reached on the basis of facts about positional augmentation, are compatible with evidence from positional neutralization concerning the special status of the "initial syllable."

Beckman $(1995,1997,1998)$ surveys a number of positional neutralization phenomena that indicate that material in initial syllables is especially resistant to

56. That is, positional faithfulness and positional augmentation constraints, in which an otherwise independent constraint is relativized to a particular, extra-phonologically salient position, are distinguished here from context-dependent markedness constraints such as ${ }^{*} \mathrm{NV}_{\text {[oral] }}$, which in a sense makes reference to the "position" $\mathrm{N}$ (cf. Kager 1999). 
featural neutralization. ${ }^{57}$ Phonological structures that are contrastive only in initial syllables include: mid vowels in Shona, nasal and long vowels in Dhangar-Kurux, round vowels in Turkic, clicks in !Xóõ, consonants with secondary articulations in Shilluk, and coda consonants with independent (non-shared) place features in Tamil (Beckman 1998:56).

As Beckman (1997:footnote 4) observes, many of the languages that she examines do not have prefixes, so that it is not possible to distinguish between rootinitial and MWd-initial syllables in many cases. However, some of the languages with special feature licensing abilities in "initial syllables," including Shona and other Bantu languages, do have prefixes - and in these languages, it is crucially the root-initial syllables that behave as strong positions. But as shown in $\$ 4.4 .1 .1$ above, restricting initial-syllable augmentation effects to root-initial syllables is incompatible with the crucially word-initial onset requirements found in languages such as Arapaho and Guhang Ifugao. How can this apparent discrepancy between positional augmentation and positional neutralization in "initial syllables" be resolved?

It seems that it may be necessary to recognize both MWd-initial and root-initial position as members of the set of psycholinguistically strong positions (roots being important in the internal structure of the lexicon, as discussed in §4.3.3). If this is the case, there should be examples of both positional augmentation in root-initial syllables and resistance to positional neutralization in MWd-initial syllables. There may in fact be an attested case of the latter, in the Bantu language Esimbi (Hyman 1988; Walker 1999, to appear). In this language, non-high vowels are banned everywhere except in the word-initial syllable, even when this syllable is a prefix. ${ }^{58}$

57. Casali $(1996,1997)$ proposes that the relevant "initial" unit with privileged status is a segment, not a syllable. However, since the effect he is trying to account for is the resistance of initial vowels to deletion (at the expense of final vowels, in $[\ldots \mathrm{V}]+[\mathrm{V} \ldots]$ contexts), his findings are compatible with the recognition of the initial syllable as a strong position. (One apparent counterexample is Nawuri (Casali 1996:25), in which front vowels surrounded by consonants become centralized, even in initial syllables, but a word-initial front vowel never becomes centralized, even when a C-final word precedes. However, granting strong-position status to word-initial segments or vowels is not truly necessary even for this case, because Nawuri could simply have a high-ranking output-output faithfulness constraint that protects initial front vowels from centralizing in derived or phrasal contexts.)

58. Esimbi is a complicated case, however, because the vowel height features that are realized in word-initial position are actually contributed by the root. Thus, root height features are the ones that are preserved (root faithfulness), but the feature contrast is permitted to appear only in word-initial position (MWd-initial syllable faithfulness-?). See also footnote 9 in Chapter 5. 
Further investigation is needed to determine whether there are positional augmentation effects specifically in root-initial syllables.

\subsection{Summary and conclusions}

This chapter has examined positional augmentation in psycholinguistically strong positions. Examples of $\mathbf{M} / \Psi$ str constraints responsible for root and initial-syllable augmentation phenomena in various languages have been presented, and various implications of these phenomena for the formal distinction between ONSET and *ONSET/X constraints, and for distinct syllable positions for onset glides and nuclear onglides, have also been discussed.

The case studies examined here support a prediction made by the model of $\mathbf{M} / \mathbf{s t r}$ constraints developed in Chapter 2: $\mathbf{M} / \Psi$ str constraints are restricted, not only by the Prominence Condition, but also by the Segmental Contrast Condition. Psycholinguistic evidence has therefore been reviewed in support of the formulation that has been given to the Segmental Contrast Condition: since the initial syllable and the root are important in early-stage word recognition, there are substantive pressures against segmental contrast neutralization in these positions. Initial-syllable augmentation constraints that manipulate onset sonority are tolerated, however, because this facilitates segmentation of the speech stream. Importantly, contrasts involving prosodic features such as stress are not blocked by the Segmental Contrast Condition, because such contrasts are not as influential in early-stage word recognition as contrasts involving segmental features are.

Finally, the proper characterization of the "initial syllable" has been considered. Examples of augmentation in, crucially, word-initial syllables have been presented, and $M W d$ has been chosen over PrWd as the relevant word-sized domain. Implications for these results with respect to cases of resistance to positional neutralization in "initial syllables" have also been explored.

While several questions concerning augmentation in psycholinguistically strong positions may remain for future investigation, a number of findings have emerged: it is important to recognize a distinction between phonetically and psycholinguistically strong positions; psycholinguistically strong positions are intimately involved in early-stage word recognition; and this involvement leads to restrictions on the nature of possible $\mathbf{M} / \Psi_{\text {str }}$ constraints that are not relevant for $\mathbf{M} / \Phi_{\text {str }}$ constraints. 


\section{Positional Augmentation and Positional Neutralization}

\subsection{Introduction}

The idea that constraints can be relativized to strong or weak positions, as with the $\mathbf{M} /$ str constraints discussed here, has previously been applied quite extensively in the OT literature to address positional neutralization phenomena. ${ }^{1}$ Three kinds of position-sensitive constraints have been introduced to account for positional neutralization: F/str constraints (positional faithfulness constraints; Selkirk 1994; McCarthy \& Prince 1995; Alderete 1995, 1999ab; Beckman 1995, 1997, 1998; Casali 1996, 1997; Lombardi 1999; Steriade 1999ab), M-Feat/wk constraints (positional feature-markedness constraints; Steriade 1997, among others), and COINCIDE constraints (alignment-type constraints for marked structure and strong positions; Zoll 1996, 1997a, 1998).

This chapter examines the relationship between positional neutralization and the positional augmentation phenomena that have been the focus of the preceding chapters. $\$ 5.2$ considers whether any of the three competing approaches to positional neutralization can be extended to account for positional augmentation

1. As noted in Chapter 1, the term 'positional neutralization' (Trubetzkoy 1939; Steriade $1993,1995)$ is used here to refer specifically to the class of phenomena in which a phonological contrast is licensed in strong positions but neutralized in weak positions. A familiar example of this kind of pattern is a language, such as Russian, that permits contrastive mid vowels in stressed syllables but not in unstressed syllables. 
effects, replacing the $\mathbf{M} / \mathbf{s t r}$ constraints that have been proposed here with either F/str constraints ( $\$ 5.2 .1)$, M-Feat/wk constraints ( $\$ 5.2 .2)$, or CoINCIDE constraints (§5.2.3) and thereby simplifying the inventory of necessary constraint types. The discussion in $\$ 5.2$ shows that none of these three approaches can in fact account for positional augmentation phenomena. Even CoINCIDE constraints, which have sometimes been described as a constraint type that handles both positional neutralization and positional augmentation effects, cannot account for positional augmentation without theoretical extensions of the very kind proposed in the model of $\mathbf{M} / \mathbf{s t r}$ constraints developed above - constraints specifically requiring prominent properties to hold of strong positions, and filters like the Prominence Condition and the Segmental Contrast Condition to prevent the generation of pathological featureneutralization constraints for strong positions. Thus, it is necessary to admit positional augmentation constraints into the universal constraint set as a distinct constraint type.

However, as discussed in $\S 5.3$, it is not surprising that fundamentally different types of constraints are responsible for positional neutralization and positional augmentation, because the two classes of phenomena are themselves qualitatively distinct-involving the avoidance of typologically marked features on the one hand and the enhancement of perceptual prominence on the other. Moreover, only attested patterns are predicted by a factorial typology that includes $\mathbf{M} / \mathbf{s t r}$ constraints and $\mathbf{F} / \mathbf{s t r}$ constraints ( $\$ 5.3 .1$ ) or by one that includes $\mathbf{M} / \mathbf{s t r}$ constraints and either CoINCIDE or M-Feat/wk constraints (§5.3.2).

\subsection{Positional neutralization in Optimality Theory}

As noted in Chapter 1, the phonologically relevant distinction between strong and weak positions first became apparent in studies of positional neutralization - the neutralization of phonological contrasts to the typologically unmarked value-where strong positions are able to resist processes of featural neutralization that affect weak positions (e.g., Trubetzkoy 1939; Steriade 1993, 1995). This section reviews three approaches that have been developed within the OT framework to account for the special ability of strong positions to resist neutralization: positional faithfulness (F/str) constraints ( $\$ 5.2 .1)$, positional featural-markedness (M-Feat/wk) constraints (\$5.2.2), and CoINCIDE constraints (§5.2.3). After each proposal is summarized, it is shown to be unable to account for positional augmentation. That is, no matter what approach is taken toward cases in which strong positions resist markedness requirements, a separate (though 
complementary) theory is needed to account for cases where strong positions are specifically affected by markedness requirements.

\subsubsection{Positional neutralization with positional faithfulness constraints}

One way to account for the special ability of strong positions to resist positional neutralization is to propose that there are faithfulness constraints that are relativized to strong positions ( $F /$ str constraints). The positional faithfulness approach emerged in work by Selkirk (1994), Alderete (1995), and McCarthy \& Prince (1995) and has been developed most fully by Beckman $(1995,1997,1998)$ and Casali $(1996$, 1997). This section reviews the theory of positional faithfulness and shows that, although it is able to account for the positional neutralization effects that it was designed for, it does not account for positional augmentation (see also Zoll 1998).

Whether a particular phonological contrast is present in a particular language is determined by the relative ranking in that language of the markedness constraints that would ban the contrast and the faithfulness constraints that would require its preservation. For example, there are languages that have contrastive mid vowels (and allow them in all positions in the word), such as Japanese. There are also languages that lack contrastive mid vowels (again in all positions), such as Quechua. In languages like Japanese, a faithfulness constraint requiring input vowel-height features to be preserved, IDENT[Vht], outranks the markedness constraint that bans mid vowels, ${ }^{*} \mathrm{MIDV}$. Therefore, when an input contains a mid vowel, the output contains a mid vowel as well. But in languages like Quechua, the ranking of these two constraints is reversed; since * MIDV is ranked higher than IDENT[Vht], even if an input were to contain a mid vowel, it would surface as something else (perhaps a high vowel, but the exact outcome might be affected by other markedness or faithfulness constraints as well, depending on their ranking). ${ }^{2}$

There is also a third type of language: one in which a particular element that is typologically marked (i.e., in that its inclusion in an inventory implies the inclusion of some other element and not vice-versa), such as the set of mid vowels, is phonologically contrastive in some strong position, but not in other positions. This

2. Recall that according to the principle of richness of the base (Prince \& Smolensky 1993), there are no language-particular restrictions on input forms (see §1.3.2, especially footnote 12). If a language has no mid vowels, we cannot simply assume that inputs never contain mid vowels, because there is no way to limit inputs in such a manner. We must instead propose a grammar for the language that would weed out surface mid vowels even if mid vowels were to appear in input forms. 
is the pattern referred to as positional neutralization. A positional-neutralization counterpart to the Japanese and Quechua examples given above would be Russian, which has contrastive mid vowels only in the strong position stressed syllable. In other syllables, that contrast is neutralized. ${ }^{3}$

The positional faithfulness approach to positional neutralization holds that there are faithfulness constraints relativized to strong positions. For example, the constraint IDENT[Vht]/o requires output vowels that appear in stressed syllables to have the same specification for vowel height as their input counterparts. The addition of this constraint to the system allows for the ranking IDENT[Vht]/o >> *MIDV >> IDENT[Vht], under which mid vowels surface in stressed syllables, but not in unstressed syllables-the pattern found in Russian-type languages.

Positional faithfulness and the positional neutralization of mid vowels (hypothetical input)

\begin{tabular}{|c||c|c|c|}
\hline /tépo/ & IDENT[Vht]/ó & $*$ MIDV & IDENT[Vht] \\
\hline \hline a. tépo & & $* * !$ & \\
\hline b. tépu & & $*$ & $*$ \\
\hline c. típu & $* !$ & & $* *$ \\
\hline
\end{tabular}

Because *MIDV dominates the general faithfulness constraint IDENT[Vht], mid vowels are banned from most positions in the language (as they are banned entirely in Quechua-type languages). However, the stressed syllable-specific faithfulness constraint IDENT[Vht]/o dominates *MIDV, which means that in stressed syllables, maintenance of an input mid vowel has the highest priority (stressed syllables in this language are thus analogous to Japanese-type languages).

Languages like Japanese and Quechua, which do not have position-sensitive neutralization (as far as mid vowels are concerned), can still be accounted for even with the inclusion of positional faithfulness constraints like IDENT[Vht]/o in the system. As long as *MIDV outranks both IDENT[Vht]/o and general IDENT[Vht], a language will ban mid vowels completely (as in Quechua). And as long as general IDENT[Vht] outranks *MIDV (no matter how IDENT[Vht]/ó is ranked), a language

3. In Russian, the basic neutralization pattern is that underlying /e/ surfaces as [i] when unstressed, whereas underlying /o/ surfaces as [a] when unstressed. The fact that the two mid vowels are handled differently by the system is separate from the fact that mid vowels are banned in unstressed syllables. 
will permit mid vowels in all positions (as in Japanese). Crucially, as (2) shows, no ranking of these three constraints ${ }^{4}$ can ever produce a language in which mid vowels are tolerated in unstressed syllables, but banned in stressed syllables. This is a desired result, since the positional neutralization of typologically marked phonological structure (such as mid vowels) is never confined to strong positions only.

(2) Grammars produced by a positional-faithfulness system

Ranking Characteristic Result

(a) $\begin{aligned} \text { M } & \gg \text { F/str }>\text { F } \\ \text { M } & >\text { F } \gg \text { F } / \text { str }\end{aligned}$

(M highest ranked) Neutralization in all positions (Quechua)

(b) F/str $>$ F $>>$ M $\quad($ F $>>M)$

$F>F / s t r>M$

Contrast in all positions

$F>M>F / s t r$

(Japanese)

4. Some versions of positional faithfulness (e.g., McCarthy \& Prince 1995 for F/Root and $\mathbf{F} / \mathbf{A f f i x}$ ) assume that faithfulness constraints are relativized to both strong and weak positions, i.e., IDENT[Vht]/ó and IDENT[Vht]/б. In order to account for the fact that neutralization never affects strong positions only, the positional constraints must be in a fixed universal ranking $F / \mathbf{s t r} \gg \mathbf{F} / \mathbf{w k}$, allowing for three different markedness rankings: $\mathbf{M}$ $\gg \mathrm{F} / \mathrm{str} \gg \mathrm{F} / \mathbf{w k}$ for neutralization in all positions; $F / \mathbf{s t r} \gg \mathrm{F} / \mathbf{w k} \gg \mathrm{M}$ for contrast in all positions; and $\mathbf{F} / \mathbf{s t r} \gg>M>\mathbf{F} / \mathbf{w k}$ for neutralization in weak positions only.

However, there are two reasons for preferring the Beckman/Casali implementation, which assumes only $\mathbf{F} / \mathbf{s t r}$ and general $\mathbf{F}$. First, this version eliminates the need for a universally fixed ranking. Although Beckman and Casali both explicitly assume that the

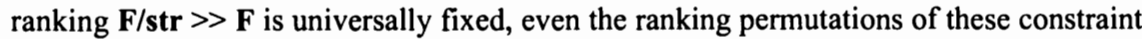
types that allow $\mathbf{F}>>\mathbf{F} / \mathbf{s t r}$ correspond to attested neutralization patterns, as shown in (2). Moreover, Struijke $(1998,2000)$ and Keer $(1999)$ argue for a crucial F $>$ F/str ranking in certain languages. It is true that languages without positive evidence to the contrary will have $\mathbf{F} / \mathbf{s t r} \gg \mathbf{F}$, since this ranking must be enforced by a "persistent bias" in order to avoid a subset problem in grammar learning (Hayes 1999b; Prince \& Tesar 1999; Smith 2000a). But

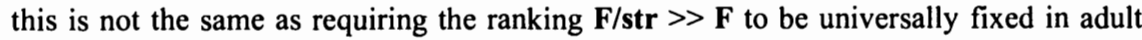
grammars.

Second, as discussed in $\S 1.3 .2$, the "weak position" counterpart to a given strong position is not always an independently identifiable class. For example, "non-initial syllables" can only be identified indirectly, as the complement of the set of initial syllables. This problem is avoided in a system that assumes only $\mathbf{F} / \mathbf{s t r}$ and general $\mathbf{F}$. 
(c) F/str $>$ M $>$ F

Neutralization in weak positions only (Russian)

Thus, the fundamental characteristics of the Beckman/Casali theory of positional faithfulness can be summarized as follows. There is a set of strong positions, identifiable because they have a special phonetic or psycholinguistic salience. Faithfulness constraints have F/str counterparts. This system allows for one of three possibilities for a given contrast: it can be neutralized throughout a language, it can be maintained throughout a language, or it can be neutralized in weak positions but maintained in strong positions.

However (as Zoll (1998) points out), the positional faithfulness system outlined above is fundamentally unable to account for positional augmentation phenomena of the sort discussed in the preceding chapters. As demonstrated in (2), no ranking of F, M, and F/str constraints can produce a language that enforces a requirement (that is, neutralizes a contrast) in strong positions and not in weak positions. But in a language with positional augmentation, that is precisely what happens: stressed syllables are forced to be heavy while unstressed syllables can be heavy or light, for example, or initial syllables are forced to have low-sonority onsets while other syllables may have any kind of onset. ${ }^{5}$

\subsubsection{Positional neutralization with positional featural markedness constraints}

A number of OT analyses have taken an alternative approach to positional neutralization phenomena, using featural markedness constraints that make reference to weak positions (M-Feat/wk). One common example is the constraint CODACOND. This constraint, an OT translation of Itô's $(1986,1989)$ Coda Condition, is designed to account for the fact that consonant place features are often neutralized specifically in coda position. CODACOND is often formulated as in (3a)

5. To be precise, a positional augmentation language is any language that enforces a requirement on strong positions that it does not enforce on weak positions. It is not always the case that the weak positions in a language with positional augmentation show a greater number of contrasts than the strong positions do-sometimes, there is a type of complementary-distribution effect, with one member of an opposition occurring exclusively in the strong position and the other member in the weak position. See $\S 5.3 .2$ for discussion. 
(see, e.g., Lombardi 2001), after Itô's original filter; this conception of CODACOND can also be stated as in (3b), to emphasize the M-Feat/wk structure of this constraint.

(3) The Coda Condition

(a) CODACOND

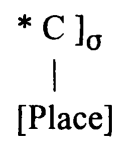

(b) CODACOND

$$
{ }^{*}[\text { Place }] / \text { Coda }
$$

In general, constraints of the M-Feat/wk type have been introduced individually, when needed for analyses of particular phonological phenomena, and not many attempts have been made to develop an explicit, typologically constrained theory of positional M-Feat constraints as a general account of positional neutralization. ${ }^{6}$ But given the empirical observation that languages that permit typologically marked elements only in certain positions will always limit them to strong positions rather than to weak positions, a constrained M-Feat theory of positional neutralization must include the restriction that M-Feat constraints can be relativized to weak positions, but not to strong positions. ${ }^{7}$ With this restriction in

6. An important exception to the lack of explicit theories of M-Feat/wk constraints is the markedness-constraint implementation of the theory of Licensing by Cue presented in Steriade (1997). Under this proposal, for every phonological feature Feat $t_{i}$ there exists a hierarchy of auditory cues to the recovery of the contrast involving that feature, ranked from best/most perceptible cues to worst/least perceptible. This hierarchy then projects a hierarchy of markedness constraints that can be schematically represented as ${ }^{*} \mathrm{Feat}_{\mathrm{i}} /[$ worst cues] $>>$

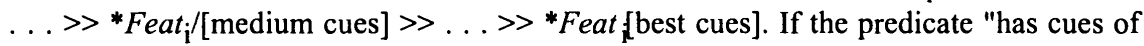
degree of goodness $G$ " is viewed as a way of defining positions, then Steriade's hierarchy is analogous to a hierarchy of positional constraints, M-Feat $/$ /weakest $>$. . > M-Feat $\mathrm{i}_{\mathrm{i}} /$ weak $>>\ldots$ > > M-Feat $\mathrm{i} /$ strong.

See also Wilson (2001) for formal extensions of Licensing by Cue. Steriade (1999ab) develops a faithfulness-based implementation. Additional discussion of Licensing by Cue is given in $\$ 2.4 .2 .3$ above.

7. This consequence is in fact a good reason to avoid the M-Feat/wk approach as a general theory of positional neutralization effects, since it brings up the problem of referring to weak positions-some of which, as noted in $\$ 1.3 .2$ and elsewhere in the current chapter, are not necessarily identifiable except as the complement of the corresponding strong position. 
place, the following factorial typology, which produces only the empirically attested patterns of neutralization of marked features, is produced.

(4) Grammars produced by a positional featural markedness system

$$
\text { Ranking Characteristic Result }
$$
(a) $\mathbf{M} \gg$ F $>>$ M-Feat $/$ wk $\quad(\mathbf{M}>>$ F $) \quad$ Neutralization in all
$\mathbf{M}>>\mathbf{M}-$ Feat $/ \mathbf{w k}>>\mathbf{F} \quad$ positions (Quechua)
M-Feat/wk $>M>$ F

(b) $\mathbf{F}>>\mathbf{M}>>\mathbf{M}-$ Feat $/ \mathbf{w k}$ (F highest ranked) Contrast in all positions F $>>M-F e a t / w k ~>>M$

(Japanese)

(c) $\mathrm{M}-$ Feat $/ \mathrm{wk}>\mathrm{F}>\mathrm{M}$

Neutralization in weak positions only (Russian)

But once again, the three classes of grammars in (4) do not include any grammar in which a phonological requirement (and thus, the neutralization of a contrast) can hold of strong positions but not of weak positions. Therefore, using only the three constraint types $\mathbf{M - F e a t / w k , ~} \mathbf{F}$, and $\mathbf{M}$, it is impossible to account for positional augmentation.

There is a sense in which the F/str and the M/Feat-wk approaches to positional neutralization are similar. Both use position-specific versions of constraints in order to ensure that a different ranking between faithfulness and markedness constraints holds in strong and weak positions. For strong positions, where the contrast is maintained, the ranking of relevant constraints is $\mathbf{F}>\mathbf{M}$ (i.e., $\mathbf{F} / \mathbf{s t r}>>\mathbf{M}$ under positional faithfulness, or $\mathbf{F}>>$ general $\mathbf{M - F e a t}$ under positional featural markedness). But for weak positions, where the contrast is neutralized, the relevant ranking is $\mathbf{M} \gg \mathbf{F}$ ( $\mathbf{M} \gg>$ general $\mathbf{F}$ under positional faithfulness, or $\mathbf{M}$ Feat/wk $>>$ F under positional featural markedness).

(5) Different relevant rankings for strong and weak positions

(a) Positional faithfulness

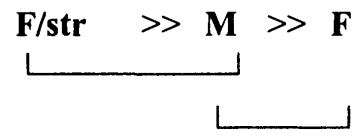

Strong positions: $\mathbf{F} \gg>\mathbf{M}$

Weak positions: $\mathbf{M}>\mathbf{F}$ 
(b) Positional featural markedness

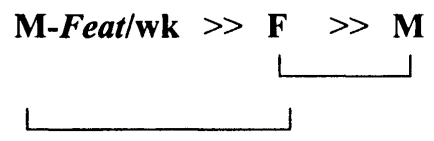

Strong positions: $\mathbf{F}>>\mathbf{M}$

Weak positions: $\mathbf{M}>\mathbf{F}$

Because these two approaches are similar in character, they face the same problem in accounting for positional augmentation. They must not produce rankings that allow positional neutralization-type phenomena (the neutralization of typologically marked features) to affect strong positions alone. To ensure this result, the position-specific constraints are limited as to the class of positions that they can target, to F/str or to M-Feat/wk respectively. As seen in (2) and (4) above, this restriction gives the correct result with respect to positional neutralization, but it also ensures that the F/str-M-F system and the M-Feat/wk-F-M system are both incapable, without further elaboration, of generating languages with positional augmentation phenomena.

The further elaboration that is needed is a way of distinguishing between the neutralization of typologically marked features, as in positional neutralization, and the enhancement of perceptual prominence, as in positional augmentation, so that constraints can be permitted that enforce the latter, but not the former, in strong positions. The proposal developed in the preceding chapters is of course a way of doing just that. Thus, either of these two approaches to positional faithfulness can be combined with the positional augmentation (M/str) constraints introduced here. As long as the $\mathbf{M} / \mathbf{s t r}$ constraints are themselves appropriately restricted, as by the Prominence Condition and the Segmental Contrast Condition, they will interact with the positional neutralization constraints only in ways that lead to classes of grammars that are attested (see §5.3). However, the point that has been demonstrated here is that both of these approaches to positional neutralization-the positional faithfulness approach and the positional featural markedness approach - are unable, on their own, to account for positional augmentation without being rendered incapable of accounting for the restricted typology of positional neutralization.

\subsubsection{Positional neutralization and augmentation with CoINCIDE constraints}

Another account of position-sensitive phonological phenomena that has been proposed is the theory of positional licensing through COINCIDE constraints (Zoll 
1996, 1997a, 1998). While the positional faithfulness and positional featural markedness theories of positional neutralization simply relativize independently attested constraint types to strong or weak positions and then derive positional effects indirectly through constraint interaction, CoINCIDE constraints are a distinct constraint type that directly relates phonological properties and strong positions. ${ }^{8}$ Nevertheless, this section shows that, just as was the case for the positional faithfulness and positional featural markedness approaches, the CoINCIDE constraints that are used to account for positional neutralization cannot be directly extended to account for positional augmentation without modifications along the lines of the proposal developed in this dissertation.

COINCIDE constraints are similar to alignment constraints (McCarthy \& Prince 1993a) in that they quantify over two categories, one universally and one existentially, and require the two categories to be associated in output forms. They differ from alignment constraints in two ways: first, they require not edge-matching but 'coincidence,' defined as a relationship of either identity or domination, and second, they are categorically rather than gradiently violable. As formulated in Zoll (1996), COINCIDE constraints quantify universally over instances of marked phonological structure, and existentially over strong positions (COINCIDE constraints of this form are abbreviated below as $\operatorname{CoINCIDE}(\alpha, s t r)$, where $\alpha$ stands for some marked phonological property). Thus, they are able to account for positional neutralization effects by requiring that a given marked structure, if it is to appear in the output at all, must appear in some strong position.

8. In Zoll (1996), CoINCIDE constraints are introduced as a distinct constraint type, formulated as in (6) below. In later work, Zoll (1998) proposes that CoINCIDE constraints can be derived through the local conjunction (Smolensky 1995, 1997) of a featuremarkedness constraint with a constraint penalizing the presence of a particular weak position. Thus, in the revised system, the coda-condition constraint "COINCIDE([CPlace], Onset)" is to be derived from the local conjunction *[CPlace] $\&_{\text {Segment }}$ NoCoDA.

However, it does not seem that the the local-conjunction approach can be successfully generalized to all instances of COINCIDE constraints. For this approach to be uniformly successful, there would have to be constraints analogous to NOCODA that ban all of the different types of weak positions. As noted above, not all weak "positions" are independently identifiable. Furthermore, it is not immediately clear to what extent there is empirical support for the existence of NOCODA-type constraints banning weak positions such as affixes or noninitial syllables. Thus, it seems preferable to maintain the Zoll (1996) formulation of CoINCIDE, which has the advantage of referring only to strong positions. (See also Fukazawa $\&$ Lombardi 2000 on problems that arise in deriving CODACOND-type constraints through local conjunction.) 
(6) General schema for CoINCIDE( $\alpha$,str) constraints (Zoll 1996:143)

COINCIDE (marked structure, strong constituent)

(i) $\forall x$ ( $x$ is marked $\rightarrow \exists y$ ( $y=$ strong constituent $\wedge$ Coincide $(x, y)$ )

- Coincide $(x, y)$ will be true if $y=x, y$ dominates $x$, or $x$ dominates $y$

(ii) Assess one mark for each value of $x$ for which (i) is false.

For example, a system with a $\operatorname{COINCIDE}(\alpha$, str $)$ constraint in the appropriate ranking can account for the positional neutralization of mid vowels in languages like Russian, where mid vowels appear only in stressed syllables. The constraint COINCIDE(MidV, ó) penalizes any mid vowel that is not in a stressed syllable. If this constraint dominates IDENT[Vht], it will cause any unstressed mid vowel to surface unfaithfully. However, this constraint will not be violated by stressed mid vowels, so they will surface faithfully (as long as IDENT[Vht] dominates *MIDV, a markedness constraint that would otherwise ban all mid vowels).

$\operatorname{COINCIDE}(\alpha, \mathbf{s t r})$ constraints and positional neutralization

\begin{tabular}{|r||c|c|c|}
\hline /tépo/ & COINCIDE(MidV, ó) & IDENT[Vht] & $*$ MidV \\
\hline \hline a. tépo & $* !$ & & $* *$ \\
\hline b. tépu & & $*$ & $*$ \\
\hline c. típu & & $* * !$ & \\
\hline
\end{tabular}

Zoll $(1996,1998)$ proposes that COINCIDE constraints can be used to account for certain cases of positional augmentation as well as for positional neutralization. However, this section shows that if positional augmentation is to be accounted for by means of coincidence, there must be a second, formally distinct class of CoINCIDE constraints. COINCIDE ( $\alpha$,str) constraints (6), the kind used to account for positional neutralization (7), cannot serve as an adequate account of positional augmentation effects, for the following two reasons.

First, (as has been argued throughout this dissertation), in positional augmentation phenomena, it is not marked structure per se that strong positions are required to have; it is one of a number of properties that enhance perceptual prominence. 
Second, in positional augmentation, it is the strong position over which some requirement holds, not the phonological feature or contrast that has a positional distribution. In the terminology introduced by Crowhurst \& Hewitt (1997), the position, not the phonological property, must be the focus of the constraint (the element associated with the universal quantifier).

This means that two classes of CoINCIDE constraints, with formally distinct constraint schemas or generalized formulations, would have to be recognized. COINCIDE $(\alpha$, str) constraints, as in (6) above, universally quantify over a certain marked phonological structure and require it to coincide with some strong position, thus accounting for positional neutralization. The new type, to account for positional augmentation, would universally quantify over a certain strong position and require it to coincide with some perceptually prominent property, as shown in (8); this type of COINCIDE constraint will be abbreviated as COINCIDE(str, $\pi$ ) (where $\pi$ is mnemonic for 'prominent property').

General formulation for COINCIDE(str, $\pi$ ) constraints

COINCIDE (strong position, prominent property)

(i) $\forall x$ ( $x$ is a strong position $\rightarrow$

$\exists y(y=$ prominent property $\wedge$ Coincide $(x, y))$

- Coincide $(x, y)$ will be true if $y=x, y$ dominates $x$, or $x$ dominates $y$

(ii) Assess one mark for each value of $x$ for which (i) is false.

Universal quantification over the strong position rather than over the phonological property is essential in accounting for positional augmentation effects. If COINCIDE constraints intended to account for positional augmentation were set up in the same form as COINCIDE( $\alpha$,str) constraints-universally quantifying over the prominent property and only existentially quantifying over the strong position - then they would be unable to enforce any property to hold of a strong position if that property was not present somewhere in the form.

For example, in a number of languages including Mongolian, the onset of a word-initial syllable can never be a liquid (\$4.2.1.2). An attempt to account for this pattern based on COINCIDE constraints with universal quantification over phonological properties would presumably invoke a constraint like the following, which states that every instance of an onset with sonority lower than that of a liquid (the perceptually prominent property) must coincide with some initial syllable (the strong position). 


$$
\begin{gathered}
\text { COINCIDE }\left(\mid \text { Onset } \mid<\mathrm{LIQ}, \sigma_{1}\right) \quad \forall x(x \text { is an onset with sonority }<\mathrm{LIQ} \rightarrow \\
\left.\exists y\left(y=\sigma_{1} \wedge \text { Coincide }(x, y)\right)\right)
\end{gathered}
$$

Crucially, a COINCIDE constraint of this form cannot be used to account for a language like Mongolian in which liquid onsets are absolutely prohibited. Since is it the phonological structure that is associated with the universal quantifier, if an input contains no non-liquid consonants, then the COINCIDE constraint in (9) is (vacuously) satisfied even with an initial liquid onset.

$$
\text { Vacuous satisfaction with putative "COINCIDE( } \pi, \text { str)" constraints }
$$

\begin{tabular}{|r||c|c|}
\hline /lala/ & CoINCIDE $\left(\mid{\left.\text { Onset } \mid<\text { LIQ }_{1}\right)}\right)$ & FAITH \\
\hline \hline a. lala & & \\
\hline b. tala & & $* !$ \\
\hline c. ala & & $* !$ \\
\hline
\end{tabular}

But words never begin with liquids in Mongolian-type languages. The actual constraint at work must therefore be one that prefers some unfaithful candidate, such as $(10 b)$ or $(10 c)$, over $(10 a)$, even when there is no non-liquid consonant in the input.

The problem of vacuous satisfaction shown in (10) is the reason why a distinct class of $\operatorname{COINCIDE}(\mathbf{s t r}, \pi)$ constraints, which universally quantify over strong positions rather than over phonological properties, must be added to the system if COINCIDE constraints are to be used in the analysis of positional augmentation.

Moreover, a theory of just what kinds of things can coincide with each other in a COINCIDE(str, $\pi)$ constraint would also be needed. It would be crucial to ensure that the $\pi$ variable could only be filled by arguments that qualify as perceptually prominent properties - that is, a counterpart of the Prominence Condition would still be required. Likewise, to prevent segmentally neutralizing augmentation phenomena from affecting psycholinguistically strong positions, a version of the Segmental Contrast Condition would also be needed.

In general, there seems to be no difference in the degree to which a theory designed to handle positional neutralization must be extended if it is to account for positional augmentation as well. A new constraint schema must be introduced, either $\mathbf{M} / \mathbf{s t r}$ or $\operatorname{CoINCIDE}(\mathbf{s t r}, \pi)$, that is different from the constraint schema adopted for positional neutralization, be it F/str, M-Feat/wk, or $\operatorname{CoINCIDE}(\alpha, \mathbf{s t r})$. Constraint filters analogous to the Prominence Condition and the Segmental 
Contrast Condition are also needed, whether it is $\mathbf{M} / \mathbf{s t r}$ or $\operatorname{CoINCIDE}(\mathbf{s t r}, \pi)$ constraints that are used in analyzing positional augmentation. In no case does an account of positional augmentation come for free.

Thus, contra Zoll $(1996,1997 \mathrm{a}, 1998)$, there is no advantage to choosing COINCIDE constraints over F/str (or M-Feat/wk) constraints in the analysis of positional neutralization based on the simplicity of an extension of the approach to positional augmentation cases. ${ }^{9}$ Further investigation is needed to determine whether it is empirically possible to distinguish between the $\mathbf{M} / \mathbf{s t r}$ and the COINCIDE(str, $\pi)$ approaches to positional augmentation. Even if $\operatorname{CoINCIDE}(\mathbf{s t r}, \pi)$ constraints ultimately prevail, the fundamental claims made in this dissertation are unchanged: substantively grounded constraint filters are an indispensable part of the theory of positional augmentation constraints.

\subsection{Implications of distinguishing positional augmentation and neutralization}

The preceding section has shown that accounts of positional neutralization, when properly constrained so that they make the right typological predictions for positional neutralization, are intrinsically unable to account for positional augmentation effects. Conversely, it is also clear that the $\mathbf{M} / \mathbf{s t r}$ constraints proposed here cannot, by themselves, account for positional neutralization. As has been demonstrated throughout the preceding chapters, $\mathbf{M} / \mathbf{s t r}$ constraints demand that certain properties hold of strong positions. They do not make any demands of weak positions, and they are antagonistic to faithfulness in strong positions (in that, if a given instance of a strong position str lacks the perceptually prominent property called for by a constraint $\mathbf{M} / \mathbf{s t r}$, then the satisfaction of $\mathbf{M} / \mathbf{s t r}$ entails a violation of faithfulness in str). Therefore, $\mathbf{M} / \mathbf{s t r}$ constraints are unable to account for either aspect of the positional neutralization pattern, the loss of contrast in weak positions or the preservation of contrast in strong positions.

In short, it is unavoidable that two separate classes of position-specific constraints be recognized: those that account for positional augmentation, namely, $\mathbf{M} / \mathbf{s t r}$ constraints, and those that account for positional neutralization, namely, one of the three systems reviewed in $\S 5.2$ above. However, this result is not surprising. Positional augmentation phenomena are fundamentally different from positional

9. In fact, Walker (to appear) suggests that both F/str-type and CoINCIDE-type constraints may be necessary to account for the privileged behavior of multiple strong positions seen in the feature-migration pattern of Esimbi. 
neutralization phenomena in that they involve the enhancement of perceptual prominence rather than the mere neutralization of phonological contrasts to the typologically unmarked value. The two goals sometimes intersect; syllables with onsets are both perceptually prominent and typologically unmarked, for example. The two considerations can also be contradictory, however, as in the case of perceptually prominent, but typologically marked, mid vowels. Therefore, the fact that no single constraint type can account for both kinds of effects is not entirely unexpected. $^{10}$

In an optimality theoretic analysis, the broader typological predictions made by any proposed constraint inventory must also be considered. In this case, it has been necessary to conclude that both positional augmentation constraints and positional neutralization constraints are present in the universal constraint inventory. What classes of languages are thereby predicted to exist? This section examines the factorial typology of a constraint inventory containing $\mathbf{M} / \mathbf{s t r}$ constraints for positional augmentation ${ }^{11}$ and one of the three kinds of constraints discussed in $\S 5.2$ for positional neutralization. A system that has $\mathbf{M}, \mathbf{F}, \mathbf{M} / \mathbf{s t r}$, and $\mathbf{F} / \mathbf{s t r}$ constraints is considered in $\S 5.3 .1$, and one that has $\mathbf{M}, \mathbf{F}, \mathbf{M} / \mathbf{s t r}$, and either COINCIDE or M-Feat/wk constraints is considered in $\S 5.3 .2$; as it turns out, both systems predict the same five general classes of grammars. $§ 5.3 .3$ then confirms that each of the five predicted grammar types does in fact occur. Thus, no incorrect typological predictions are made when both positional augmentation constraints and positional neutralization constraints are included in CON.

10. Another proposal that attempts to unify positional neutralization and certain instances of what would under the present system be classified as positional augmentation is that of Dresher \& van der Hulst (1998). Dresher and van der Hulst argue that elements that are heads of prosodic (or segmental) units tolerate, and in some cases require, more complexity than non-head (dependent) elements, leading to positional neutralization and positional augmentation effects respectively. However, it does not seem that the notion of head versus non-head can be extended to all pairs of strong and weak positions-for example, the root, a strong position by virtue of its role in speech perception, is generally not a (morphosyntactic) head in derived forms. Even more problematic for this approach is the fact that not all attested cases of positional augmentation do involve an increase in complexity. For example, one kind of positional augmentation effect is the preference for low-sonority consonants in the onsets of stressed or initial syllables. Theories that try to derive markedness from complexity of structure (e.g., Rice 1992) generally propose that obstruents, as the less marked consonants, have less structural complexity.

11. The same factorial typologies would also be found if positional augmentation constraints of the form COINCIDE( $(\mathbf{s t r}, \pi)$ were assumed in place of $\mathbf{M} / \mathbf{s t r}$ constraints, since the two constraint types are both violated only if the strong position they refer to fails to have the prominent property they invoke. 


\subsubsection{Factorial typology with $\mathrm{M} / \mathrm{str}$ and $\mathrm{F} / \mathrm{str}$ constraints}

To investigate the typological implications of a constraint inventory that contains general $\mathbf{M}$, general $\mathbf{F}, \mathbf{M} / \mathbf{s t r}$, and $\mathbf{F} / \mathbf{s t r}$ constraints, consider a representative constraint set containing one of each of these types of constraint. The crucial case is one in which as many of these constraints conflict with each other as possible. Such a case can be constructed as follows. Let $\mathbf{M}$ and $\mathbf{F}$ be conflicting constraints (such as $\mathbf{M}=*$ MIDV and $\mathbf{F}=$ IDENT[hi]); let $\mathbf{F} / \mathbf{s t r}$ be a positional version of $\mathbf{F}$ (such as IDENT[hi]/o); and let $\mathbf{M} / \mathbf{s t r}$ be an augmentation constraint that is relativized to the same position as F/str and conflicts with it (such as $\left.\left[{ }^{*} \mathrm{PEAK} / \mathrm{HIGHV}\right] / \sigma\right){ }^{12} \mathrm{~A}$ factorial typology of these four constraints includes 4 ! or 24 distinct rankings. Nevertheless, as shown in (11), only five basic language types are predicted to occur: languages with full contrast; languages with full neutralization; languages with positional neutralization; and two kinds of languages with positional augmentation, those with contrast maintained outside the strong position and those with complementary distribution in strong and weak positions.

12. Note that $\mathbf{M} / \mathbf{s t r}\left(\left[{ }^{*} \mathrm{PEAK} / \mathrm{HIGHV}\right] / \sigma\right)$ conflicts with $\mathbf{M}\left({ }^{*} \mathrm{MIDV}\right)$ in this example (as long as IDENT[low] is ranked high enough to prevent an input high vowel from being realized as a low vowel). Whether or not a given $\mathbf{M} / \mathbf{s t r}$ constraint will conflict with some general $\mathbf{M}$ constraint depends on the nature of the $\mathbf{M} / \mathbf{s t r}$ constraint in question. This point is discussed further in $\S 5.3 .3$ below. 
(11) Factorial typology: M/str and F/str constraints

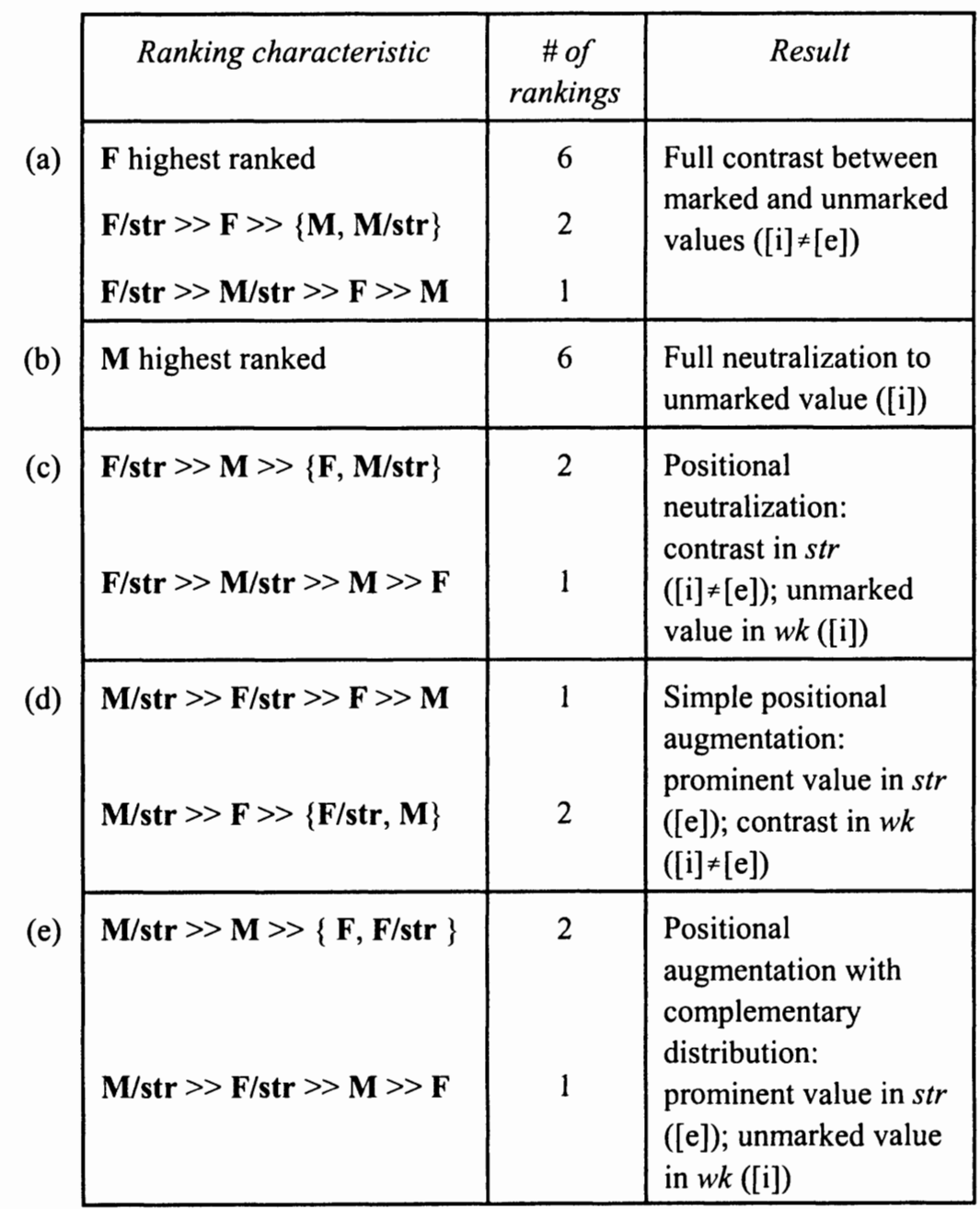

In the rankings shown in (11a), where no $\mathbf{M}$ constraint is active, the language has full contrast in all positions (note that full contrast is possible even if $\mathbf{M} / \mathbf{s t r}$ dominates general $\mathbf{F}$, as long as $\mathbf{F} / \mathbf{s t r}$ dominates $\mathbf{M} / \mathbf{s t r}$, thereby protecting the contrast in the strong position). Another simple case is that of $(11 \mathrm{~b})$ : if general $\mathbf{M}$ is highest ranked, then the contrast is neutralized to the unmarked value in all positions regardless of the rankings among the other three constraints. Languages of these two general types are well attested in the literature. 
More intricate interactions involving the positional constraints are seen in the three remaining grammar types. In (11c), what is crucial is that $\mathbf{F} / \mathbf{s t r}$ dominates both markedness constraints, allowing the contrast to be maintained in the strong position, but general $\mathbf{M}$ dominates general $\mathbf{F}$, leading to neutralization to the unmarked value outside the strong position-this is classical positional neutralization, another well-attested language type.

The rankings in (11d-e) all produce languages with positional augmentation effects (neutralization to the perceptually prominent value in the strong position), because in each of these cases, $\mathbf{M} / \mathbf{s t r}$ is highest ranked, dominating all three of the constraints that conflict with it. The difference between (11d) and (11e) is in the relative ranking of general $\mathbf{F}$ and general $\mathbf{M}$. In (11d), the ranking $\mathbf{F}>\mathbf{M}$ allows contrast outside of the strong position. In (11e) this ranking is reversed, leading to neutralization to the unmarked value outside the strong position. As discussed further in $\S 5.3 .3$ below, these two language types are likewise empirically attested.

\subsubsection{Factorial typology with M/str and CoINCIDE or M-Feat/wk constraints}

As shown in $\S 5.2$, other ways to model positional neutralization are systems that assume M-Feat/wk constraints or $\operatorname{CoINCIDE}(\alpha, s t r)$ constraints. This section examines the factorial typology of a constraint set including $\mathbf{M} / \mathbf{s t r}$ constraints and one of these two types of positional-neutralization constraints. This factorial typology predicts the same five grammar types as the system with F/str constraints considered above.

Since analogous M-Feat/wk and COINCIDE constraints, such as *MIDV/ $/ \widetilde{\sigma}$ and COINCIDE(MidV,ó), have the same pattern of constraint violation - each of the two is violated whenever there is a mid vowel in an unstressed syllable and satisfied otherwise - they can be conflated for the purposes of this discussion. Therefore, the abbreviation $\mathbf{M} / \mathbf{w k}$ will be used to stand for either M-Feat/wk constraints or COINCIDE constraints in the factorial typology examined below.

As in §5.3.1, the most instructive case to consider is one in which the four constraints conflict among themselves as much as possible. Again, let $\mathbf{M}$ and $\mathbf{F}$ be the conflicting constraints $\mathbf{M}={ }^{*} \mathrm{MIDV}$ and $\mathbf{F}=\operatorname{IDENT}[\mathrm{hi}$; then let $\mathbf{M} / \mathbf{w k}$ be a positional version of $\mathbf{M}$ (such as *MIDV/ ) or the analogous CoINCIDE constraint (such as COINCIDE(MidV,ó)); and let $\mathbf{M} / \mathbf{s t r}$ be an augmentation constraint that is antagonistic to $\mathbf{F}$ and affects the strong position related to $\mathbf{M} / \mathbf{w k}$ (such as [*PEAK/HIGHV]/ó). 
(12) Factorial typology: $\mathbf{M} / \mathbf{s t r}$ and $\mathbf{M} / \mathbf{w k}$ constraints

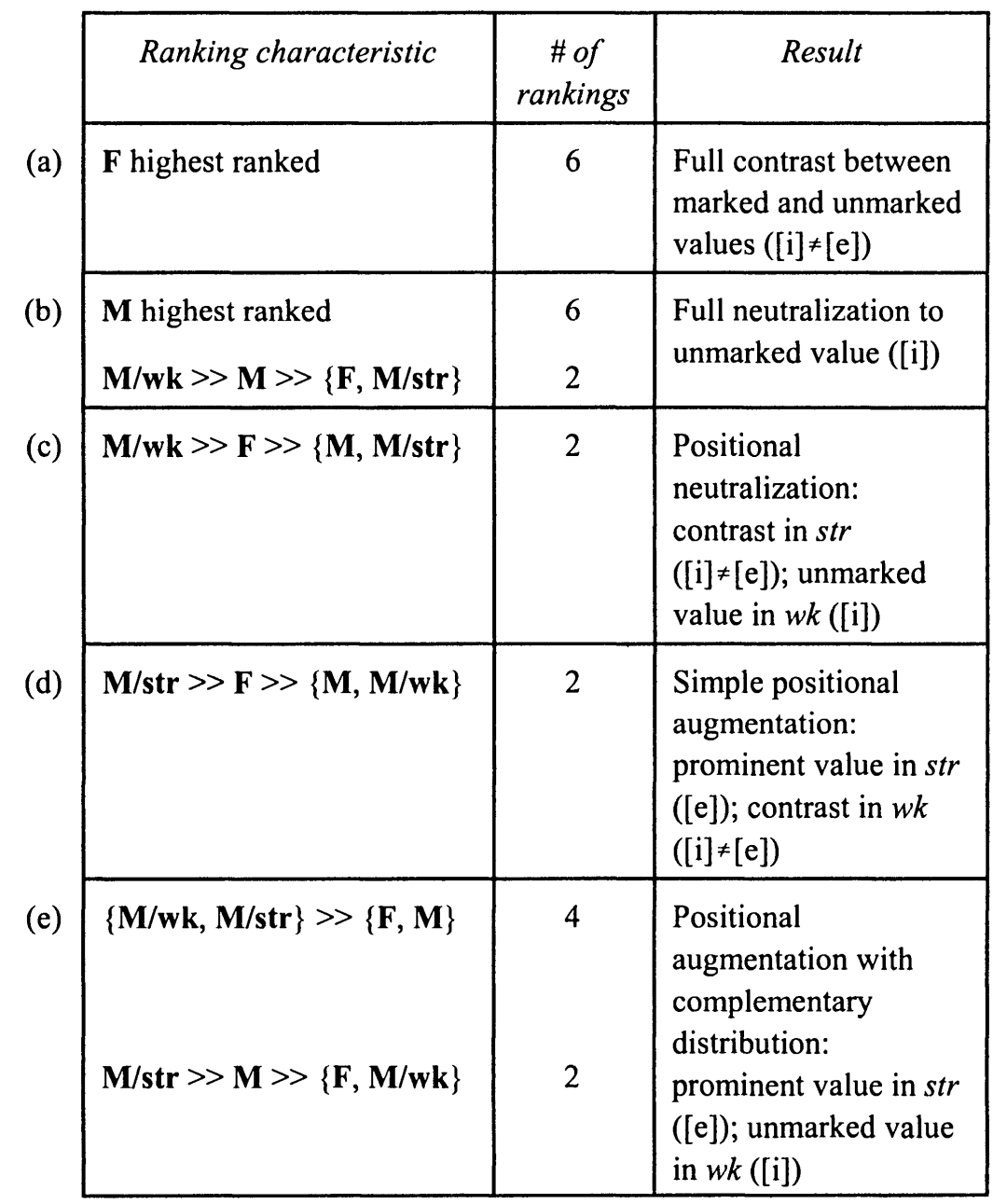

The same five grammar types are predicted as in the typology considered in $\S 5.3 .1{ }^{13}$ If $\mathbf{F}$ is highest ranked, there will be full contrast in all positions (12a).

13. A comparison of the \# of rankings columns in (11) and (12) shows that the two systems predict different numbers of rankings to fall under each of the five general grammar types. Some researchers have proposed that the number of distinct rankings that result in a particular pattern should correlate with the observed frequency of the pattern (see, e.g., Anttila 1997). If so, then this might provide a way to distinguish empirically between the 
Whenever $\mathbf{M}$ dominates both of the conflicting constraints ( $\mathbf{F}$ and $\mathbf{M} / \mathbf{s t r}$ ), there will be neutralization to the unmarked value in all positions (12b). If $\mathbf{M} / \mathbf{w k}$ dominates $\mathbf{F}$, but $\mathbf{F}$ dominates $\mathbf{M}$ and $\mathbf{M} / \mathbf{s t r}$, there will be neutralization to the unmarked value in the weak position, but contrast will be maintained in the strong position: positional neutralization (12c). Finally, there are again two kinds of positional augmentation languages (12d-e): any ranking in which $\mathbf{M} / \mathbf{s t r}$ dominates $\mathbf{F}$ and $\mathbf{M}$ will result in neutralization to the perceptually prominent value in the strong position (positional augmentation). The difference between the two patterns lies in whether or not $\mathbf{F}$ dominates both of the markedness constraints that are relevant for the weak position, $\mathbf{M}$ and $\mathbf{M} / \mathbf{w k}$. In rankings where $\mathbf{F}$ dominates both of these constraints, then contrast is maintained in the weak position (12d). However, if either $\mathbf{M}$ or $\mathbf{M} / \mathbf{w k}$ dominates $\mathbf{F}$, then the weak position is neutralized to the typologically unmarked value (12e).

\subsubsection{Positional augmentation and complementary distribution}

As the previous two subsections have shown, a constraint inventory that includes the constraints needed to derive both positional augmentation and positional neutralization phenomena will allow five types of grammars. Of these five, the existence of the first three types - contrast between members of a phonological opposition in all positions, neutralization to the unmarked value in all positions, and positional neutralization (neutralization to the unmarked value in weak positions but contrast in strong positions)-is well known.

The two remaining language types give rise to positional augmentation phenomena such as those that have been discussed in the preceding chapters. In both types, the strong position undergoes neutralization to the perceptually prominent member of a phonological opposition; this is the defining characteristic of a positional augmentation system. The crucial difference between the two types lies in whether the weak position maintains a phonological contrast $(11 \mathrm{~d}) /(12 \mathrm{~d})$, or

F/str and $\mathbf{M} / \mathbf{w k}$ approaches to positional faithfulness. However, work on the formal learnability of OT grammars (especially Smolensky 1996, Hayes 1999b, Prince \& Tesar 1999, and Smith 2000a) has shown that certain types of rankings are chosen by the learner only when positive evidence for those rankings is encountered. Since the choice between two rankings that would produce the same language type is therefore not necessarily random, it may not be meaningful simply to relate the frequency of a particular phonological pattern to the raw number of constraint rankings that would be compatible with such a pattern. 
whether it undergoes neutralization to the unmarked member of the contrast $(11 \mathrm{e}) /(12 \mathrm{e})$.

Many of the languages examined in chapters 3 and 4 are of the $(11 d) /(12 d)$ type, which might be dubbed "simple positional augmentation." In these languages, the $\mathbf{M} / \mathbf{s t r}$ constraint enforces neutralization of a contrast to the perceptually prominent value in the strong position, but contrast is maintained in the weak position. This can be seen for example in Arapaho ( $\$ 4.2 .1 .1$ ), in which initial syllables must have onsets, but other syllables may have onsets or lack them. Other examples are Chamicuro (\$3.4), in which onset consonants must have a supralaryngeal Place specification, but other consonants may have or lack such a specification; Zabiče Slovene (§3.2.1.3), where stressed short-V syllables may not contain high vowels, but other short-V syllables may contain high, mid, or low vowels; and the languages in $\S 4.2 .1 .2$, where initial syllables may not contain highsonority onsets, but other syllables may contain onsets of high or low sonority.

Examples of the $(11 \mathrm{e}) /(12 \mathrm{e})$ language type are also attested. This pattern involves neutralization in both strong and weak positions. In strong positions, the phonological contrast in question is neutralized to the perceptually prominent value. In weak positions, the contrast is neutralized to the typologically unmarked value. This pattern was labeled "positional augmentation with complementary distribution" in (11) and (12) above, because in cases where the perceptually prominent value is distinct from the typologically unmarked value, the two values will surface only in the strong position and only in the weak position respectively; hence, they will be in complementary distribution. This kind of pattern can be seen in Niuafo'ou loanwords (\$3.2.2.3), where high vocoids in hiatus are syllabified as vowels when the following vowel is stressed (to satisfy the M/str constraint [ $\left.{ }^{*} \mathrm{ONSET} / \mathrm{GLI}\right] / \sigma$ ) but as glides elsewhere (to satisfy ONSET). Thus, glides and high vowels are in complementary distribution, and the conditioning environment is the structural relationship of the high vocoids to the strong position stressed syllable. (See also de Lacy 2000, to appear for discussion of this point.) Other examples of this pattern are found in languages that have positional augmentation in stressed syllables that is related to vowel prominence, along with reduction of vowels outside of stressed syllables to typologically unmarked values-mid vs. high vowels (Chamorro; Chung 1983, Crosswhite 1999a); long vs. short vowels (Icelandic; Einarsson 1949; and Coatzospan Mixtec; Gerfen 1999:§3.6.1.1); and full vs. reduced vowels. Another plausible example would be a language that requires all onset consonants to have a supralaryngeal Place specification, like Chamicuro (§3.4; Parker 1994, 2000), while banning supralaryngeal Place in coda consonants, like Kelantan Malay (Teoh 1988; Lombardi 2001).

It should be emphasized that not all $\mathbf{M} / \mathbf{s t r}$ constraints will participate in an observable complementary distribution pattern even when ranked as in $(11 \mathrm{e}) /(12 \mathrm{e})$. 
In cases where the perceptually prominent member of a phonological opposition is also the typologically unmarked value, then the "complementary distribution" ranking will produce a language that looks like the $(11 b) /(12 b)$ case: neutralization to the unmarked value in all positions. This is because the "complementary distribution" ranking causes strong positions to be neutralized to the perceptually prominent value and weak positions to be neutralized to the typologically unmarked value, so in cases where the same member of the opposition is both perceptually prominent and typologically unmarked, the strong and weak positions will be neutralized to the same value. An example of an $\mathbf{M} / \mathbf{s t r}$ constraint with this effect is ONSET/o, since syllables with onsets are both more perceptually prominent and less typologically marked than syllables without onsets.

\subsection{Summary and conclusions}

This chapter has explored the interaction of $\mathbf{M} / \mathbf{s t r}$ constraints with other kinds of positional constraints. Since positional augmentation involves the enhancement of perceptual prominence in strong positions, whereas positional neutralization involves the elimination of typologically marked elements in weak positions, fundamentally distinct constraint types are involved in each case, and both must be included in the universal constraint set. The factorial typology predicted by such a constraint set is consistent with the kinds of languages that are empirically attested: languages with full contrast, full neutralization, positional neutralization, simple positional augmentation, and (for $\mathbf{M} / \mathbf{s t r}$ constraints where the perceptually prominent value of a contrast is not the same as the typologically unmarked value) positional augmentation with complementary distribution. 


\section{Conclusions, Implications, and Future Directions}

Phonologically "strong" positions are traditionally distinguished by their special ability to resist featural neutralization phenomena that affect other positions (Trubetzkoy 1939; Steriade 1993, 1995; Beckman 1995, 1997, 1998; Casali 1996, 1997). However, there are certain phonological requirements that sometimes hold of strong positions specifically, including requirements that stressed syllables be heavy, that long vowels be high in sonority, that initial syllables have onsets, or that roots bear stress. This dissertation has presented a theory of strong position-specific requirements that accounts for those that exist while excluding those that do not. Specifically, it is proposed that certain substantive considerations determine when a markedness constraint can be relativized to a strong position, and that the substantive considerations in question are implemented as constraint filters that use extra-phonological information to screen formally possible constraints and determine which of them are to be included in CON.

Thus, in the model developed here, phonological requirements for strong positions are imposed by $\mathbf{M} / \mathbf{s t r}$ constraints. However, M/str constraints are legitimate constraints only if they call for strong positions to have perceptually prominent properties, a restriction enforced by the constraint filter known as the Prominence Condition. M/str constraints on psycholinguistically strong positions are further restricted by another filter, the Segmental Contrast Condition, which reflects the importance that these positions have in early-stage word recognition; not even the addition of perceptually prominent properties to these positions is advantageous if doing so would neutralize psycholinguistically useful segmental contrasts without facilitating left-edge demarcation. 
Several topics closely related to the core empirical phenomena examined in the preceding chapters will be interesting to investigate further in light of the findings assembled here. One such topic is the status of tone-related augmentation constraints. First, as noted in \$4.3.4.2, the psycholinguistic evidence to date concerning the relationship of tonal contrasts to early-stage word recognition has not yet converged on a firm conclusion. If it is in fact the case that tone, like stress, is less important in early-stage word recognition than segmental contrasts are, at least in some languages, then this model predicts that there should be tone-related augmentation effects in psycholinguistically strong positions, in addition to those already identified in phonetically strong positions. Another question concerning tone is the extent to which augmentation constraints exist that refer to tones other than $\mathrm{H}$; for example, attraction of $\mathrm{L}$ tones to strong positions has been discussed by, e.g., Kang (1997) and Yip (2000).

Another goal to be pursued in future work is a deeper understanding of the nature of the set of strong positions and the role of these positions in phonology generally (i.e., in positional neutralization as well as in positional augmentation). In addition to the five positions investigated here-stressed syllable, long vowel, onset/released consonant, initial syllable, and root-what other strong positions are there, and what kinds of positional augmentation do they undergo? As observed in $\S 1.3 .2$, heads of prosodic constituents both larger and smaller than the PrWd, in particular secondary-stress syllables (foot heads) and phrasal heads, are probably also included in the set of strong positions, and the lexical category noun is probably included as well. Also, there may be justification for recognizing rootinitial syllable and MWd-initial syllable as distinct strong positions (\$4.4.2). But are there other strong positions beyond even these? For example, the final syllable is sometimes identified as a position that is able to resist positional neutralization (Trubetzkoy 1939; Steriade 1993; Hyman 1998). Is this position actually a strong position, and if so, which kind? Steriade (1993) observes that final lengthening is a common phenomenon and suggests that phonetic length accounts for the resistance of final position to featural neutralization. On the other hand, Brown \& McNeill (1969) and Nooteboom (1981) suggest that word-final material appears to be more useful than word-medial material in word recognition, so this position may have some degree of psycholinguistic importance.

Moreover, since the strong positions themselves are granted their special status on the basis of substantive factors related to phonetic salience and psycholinguistic importance, are these positions in fact supplied as primitive universal phonological building blocks, as assumed in $\$ 2.2$ ? Or alternatively, are even the strong positions identified through the action of constraint filters? In such an approach, the relativization of any constraint to any position would be formally possible, but positional constraints relativized to any non-salient positions would be rejected by 
a substantively based filter. The identification of strong positions by means of a constraint filter is an attractive idea in some respects, because it links the substantive basis for strong-position status more directly to the substantive grounding of particular constraints: both are achieved through filters. However, it is not entirely clear that this alternative approach to strong positions is feasible. It does not seem reasonable to expect that any arbitrary position ("fifth syllable," "second element of a cluster" ... ) can be made available to a relativized constraint (see also §1.3.2). It may be necessary to recognize the set of strong positions as part of the universal inventory of basic phonological elements simply to allow constraints to refer to these positions at all. In any case, a characterization of the means by which the set of strong positions becomes available to the formal grammar is an interesting and important point to pursue.

The most fundamental question related to the topics investigated in the preceding chapters is this: what is the relationship between formal and substantive aspects of phonological theory? In other words, how direct a contribution do substantive pressures make to phonological patterning, and to what extent is an abstract formal system necessary? A number of the phenomena that have been discussed here have implications for this question, supporting the approach to interactions between formal and substantive considerations that is taken in the Schema/Filter model.

Optimality Theory, which seeks to explain phonological phenomena on the basis of interactions among ranked and violable constraints, provides a way for substantive concerns to play a more direct role in the grammar than was possible in rule-based theories. For example, a particular markedness constraint such as *VoicedOBSTRUENT can be seen to codify the articulatory and/or perceptual difficulty inherent in a particular phonological structure (in this case, voiced obstruents). In rule-based theories of phonology, rules were sometimes said to exist or apply because of some kind of "functional pressure," but there was no way of encoding that kind of pressure in the formulation of the rule itself. In OT, on the other hand, (many of) the constraints themselves literally represent individual functional pressures.

Following this line of reasoning to its ultimate conclusion, one might even ask whether there is any need for a system of formal entities in the constraint set at all. Perhaps all constraints arise directly from substantive pressures. In a sense, such a system can be seen as a null hypothesis: no abstract structure intervenes between the substantive pressures and the system of constraints, because the constraints are the substantive pressures.

Nevertheless, a number of the results discussed in this dissertation show that the phonology does indeed deal with constraints and phonological structures that are more abstract than substantive pressures or concrete properties of the speech 
stream. For example, while the constraint ONSET and the sonority-sensitive *ONSET/X subhierarchy perform a related function-ensuring that syllables have well-formed onsets-the specific formulations of ONSET and *ONSET/X are distinct, and as a result, the phonological structures that satisfy the two are different (§2.3.2.3.3, §3.2.2.3, §4.2.1.2.4). First, ONSET is satisfied by the presence of any pre-peak segment, regardless of its sonority or its precise position in prosodic structure (true onset versus nuclear onglide), but the *ONSET/X constraints are sensitive both to sonority and to syllabic position. Furthermore, ONSET can be variably ranked with respect to the constraints in the *ONSET/X subhierarchy, so it must not itself be a member of that subhierarchy. Therefore, ONSET and *ONSET/X, while related to, and perhaps existing in response to, the same kinds of functional pressure, are formally distinct in the phonological system.

Another result examined here that necessitates recognizing abstract, formal elements is the relationship of phonetically strong positions (e.g., stressed syllable, onset/released consonant, and long vowel) to positional augmentation processes. The Licensing-by-Cue approach (Steriade 1993, 1995, 1997, 1999ab) proposes that phonetically strong positions have a special ability to license phonological contrasts, and thereby avoid positional neutralization, because they possess salient cues to the recovery of particular phonological contrasts. This claim is well supported by the empirical facts of positional neutralization cases. However, the null hypothesis--that the only thing special about phonetically strong position $P$ is its salient cues $x$ and $y$, and that it is these cues themselves that have a special ability to license contrast, so that there is nothing intrinsically special about position $P$ at all-cannot be maintained when the patterns of positional augmentation in these positions are considered. Crucially, phonetically strong positions are eligible for any kind of augmentation process, even one that is unrelated to the special cues possessed by the position in question. For example, the position stressed syllable is a phonetically strong position by virtue of its salient cues for vowel feature contrasts, and so resistance to positional neutralization by stressed syllables typically involves vowel features (or suprasegmentals like tone or length that are realized on vowels), just as the Licensing-by-Cue approach would predict. But positional augmentation constraints that target stressed syllables include those demanding onsets or low-sonority onsets (\$3.2.2). That is, from the point of view of the phonological system, the element stressed syllable does have a special phonological status that can be referred to abstractly, as by augmentation constraints, and is not tied directly to its salient perceptual cues. Instead, the presence of those cues gives the position its abstract, formal status as a strong position. Filters on positional faithfulness constraints $(\$ 2.4 .2 .3)$ ensure that any feature-licensing abilities of the phonetically strong position stressed syllable are indeed related to its special salient cues. But because there are no filters that 
specifically restrict the formal possibilities for augmentation constraints on phonetically strong positions (beyond the Prominence Condition, which ensures that all positional markedness constraints are augmentation constraints), the stressed syllable is eligible for all manner of positional augmentation constraints. If there were no abstract level at which this position qualified as a strong position, irrespective of its particular phonetic attributes, then there would be no way in which augmentation constraints unrelated to those phonetic attributes could identify it as a strong position.

Finally, it is necessary to draw a formal distinction between the constraint filters and EVAL, the component of the grammar that chooses the optimal output candidate for each input. This is because the substantive pressures that are instantiated in the constraint filters have no direct effect on the selection of optimal output forms. For example, the Prominence Condition is a constraint filter that prevents non-prominence-enhancing constraints from having $\mathbf{M} / \mathbf{s t r}$ counterparts. However, it is not the case that strong positions are never affected by markedness constraints that happen not to enhance perceptual prominence. If a general markedness constraint such as ${ }^{*} \mathrm{MIDV}$, which is not an augmentation constraint (§2.3.1), dominates all vowel-height faithfulness constraints in the language, it will ban contrast in all positions, including strong positions such as stressed syllables, long vowels, initial syllables, and roots. The Prominence Condition does not prevent this; in fact, languages without any mid vowels are attested (and likewise for other feature-markedness constraints). Similarly, the Segmental Contrast Condition is a constraint filter that bans positional augmentation constraints whose satisfaction would require the neutralization of (non-edge-demarcating) segmental contrasts in psycholinguistically strong positions. However, this does not mean that such a neutralization might not come about indirectly, as a consequence of constraint ranking. For example, the $\left[{ }^{*} \mathrm{PEAK} / \mathrm{X}\right] \sigma_{1}$ subhierarchy is banned by the Segmental Contrast Condition. But in a language where stress always falls on the initial syllable, and low-sonority nuclei are banned by [ $\left.{ }^{*} \mathrm{PEAK} / \mathrm{HIGHV}\right] / \sigma$, the initial syllable-because it is also the stressed syllable-will support no contrast between high and mid vowels. The Segmental Contrast Condition has no power to prevent this kind of pattern. Crucially, then, it is not "non-prominence-enhancing neutralization in strong positions" and "contrast neutralization in psycholinguistically strong positions" that are blocked by the Prominence Condition and the Segmental Contrast Condition respectively. Instead, it is constraints designed to achieve such ends directly that the constraint filters block.

These considerations show that the substantive pressures represented by the Prominence Condition and the Segmental Contrast Condition are not general forces that can have an effect on any aspect of phonology. Rather, these substantive pressures have a specific point of interaction with the phonological system: they 
prevent the inclusion of certain constraints, whose satisfaction would directly oppose the substantive pressures behind these filters, in CoN.

Thus, the Schema/Filter model of CON developed in this dissertation serves as an explicit framework for a theory of $\mathbf{M} / \mathbf{s t r}$ constraints, and more broadly, it is a way of modeling the complexity of the relationship between the abstract, formal behavior of the phonological system and the substantive pressures that underlie many of the constraints in the system. Crucially, while incorporating substantive pressures into the system (in the filters), this model maintains the view of phonology as a formal system. Such a model is supported by the evidence for formal, abstract patterning in phonology that has emerged over the course of this investigation of phonological augmentation in prominent positions. 


\section{Bibliography}

Alderete, John. 1995. Faithfulness to prosodic heads. Ms, University of Massachusetts, Amherst. ROA \#94 [http://roa.rutgers.edu].

1999a. Head dependence in stress-epenthesis interaction. In Ben Hermans and Marc van Oostendorp, eds., The Derivational Residue in Phonological Optimality Theory. Amsterdam: John Benjamins, 29-50. ROA \#453 [http://roa.rutgers.edu].

- 1999b. Morphologically Governed Accent in Optimality Theory. Doctoral dissertation, University of Massachusetts, Amherst. [New York: Routledge, 2001.]

- 2001. Root-controlled accent in Cupeño. Natural Language and Linguistic Theory 19: 455-502. ROA \#253 [http://roa.rutgers.edu, 1998].

Altmann, Gerry T. M. 1990a. Cognitive models of speech processing: An introduction. In G.T.M. Altmann, ed., 1-23.

- ed. 1990b. Cognitive Models of Speech Processing: Psycholinguistic and Computational Perspectives. Cambridge, Mass: MIT Press.

Anttila, Arto. 1997. Deriving variation from grammar. In Frans Hinskens, Roeland van Hout, and W. Leo Wetzels, eds., Variation, Change and Phonological Theory. Amsterdam: Benjamins, 35-68. ROA \#63 [http://roa.rutgers.edu].

—_ 2002. Morphologically conditioned phonological alternations. Natural Language and Linguistic Theory 20: 1-42. ROA \#425 [http://roa.rutgers.edu].

Archangeli, Diana. 1984. Underspecification in Yawelmani Phonology and Morphology. Doctoral dissertation, MIT. [New York: Garland, 1988.]

Archangeli, Diana, and Douglas Pulleyblank. 1994. Grounded Phonology. Cambridge, Mass.: MIT Press.

Archangeli, Diana, and Keiichiro Suzuki. 1997. The Yokuts challenge. In I. Roca, ed., 197-226.

Aronoff, Mark. 1976. Word Formation in Generative Grammar. Cambridge, Mass.: MIT Press.

Arvaniti, Amalia, D. Robert Ladd, and Ineke Mennen. 1998. Stability of tonal alignment: The case of Greek prenuclear accents. Journal of Phonetics 26: 3-25. 
Baayen, R. Harald, Cristina Burani, and Robert Schreuder. 1996. Effects of semantic markedness in the processing of regular nominal singulars and plurals in Italian. Yearbook of Morphology 1996: 14-34.

Baayen, R. Harald, Ton Dijkstra, and Robert Schreuder. 1997. Singulars and plurals in Dutch: Evidence for a parallel dual-route model. Journal of Memory and Language 37: 94-117.

Baković, Eric. 1998. Unbounded stress and factorial typology. In Ron Artstein and Madeline Holler, eds., RuLing Papers I: Working Papers from Rutgers. New Brunswick, NJ: Rutgers University Department of Linguistics. ROA \#244 [http://roa.rutgers.edu].

Barnes, Janet. 1996. Autosegments with three-way lexical contrasts in Tuyuca. International Journal of American Linguistics 62: 31-58.

Beckman, Jill N. 1995. Shona height harmony: Markedness and positional identity. In J. Beckman, L. Walsh Dickey, and S. Urbanczyk, eds., 54-75.

1997. Positional faithfulness, positional neutralisation and Shona vowel harmony. Phonology 14: 1-46.

- 1998. Positional Faithfulness. Doctoral dissertation, University of Massachusetts, Amherst. [New York: Garland, 1999.]

Beckman, Jill N., Laura Walsh Dickey, and Suzanne Urbanczyk, eds. 1995. Papers in Optimality Theory. University of Massachusetts Occasional Papers 18. Amherst, Mass.: GLSA.

Beckman, Mary E. 1986. Stress and Non-Stress Accent. Dordrecht: Foris.

Benua, Laura. 1995. Identity effects in morphological truncation. In J. Beckman, L. Walsh Dickey, and S. Urbanczyk, eds., 77-136.

-1997. Transderivational Identity: Phonological Relations Between Words. Doctoral dissertation, University of Massachusetts, Amherst. [New York: Garland, 2000.]

Bird, Sonya, Andrew Carnie, Jason D. Haugen, and Peter Norquest, eds. 2000. Proceedings of WCCFL XVIII. Somerville, Mass.: Cascadilla Press.

Blevins, Juliette. 1994. A place for lateral in the feature geometry. Journal of Linguistics 30: 301-348.

Bloch, Bernard, and George L. Trager. 1942. Outline of Linguistic Analysis. Baltimore, Md.: Waverly Press.

Bloomfield, Leonard. 1933. Language. New York: Holt.

Boersma, Paul. 1998. Functional Phonology. The Hague: Holland Academic Graphics.

Bolognesi, Roberto. 1998. The Phonology of Campidanian Sardinian: A Unitary Account of a Self-Organizing Structure. Doctoral dissertation, University of Amsterdam. Amsterdam: Holland Institute of Generative Linguistics.

Bond, Zinny S. 1999. Slips of the Ear: Errors in the Perception of Casual Conversation. San Diego, Cal.: Academic Press.

Booij, Geert. 1995. The Phonology of Dutch. Oxford: Oxford University Press.

Borowsky, Toni, Junko Itô, and Ralf-Armin Mester. 1984. The formal representation of ambisyllabicity: Evidence from Danish. In Charles Jones and Peter Sells, eds., Proceedings of NELS 14. Amherst, Mass.: GLSA, 34-48. 
Boudelaa, Sami, and William D. Marslen-Wilson. 2000. Non-concatenative morphemes in language processing: Evidence from Modern Standard Arabic. Proceedings of Spoken Word Access Processes 2000.

Bouma, Herman, and Don G. Bowhuis, eds. 1984. Attention and Performance X: Control of Language Processes. Proceedings of the Tenth International Symposium on Attention and Performance. Hillsdale, NJ: Lawrence Erlbaum Associates.

Bradley, Dianne. 1980. Lexical representation of derivational relation. In Mark Aronoff and Mary-Louise Kean, eds., Juncture. Saratoga, Cal.: Anma Libri, 37-55.

Breen, Gavan, and Rob Pensalfini. 1999. Arrernte: A language with no syllable onsets. Linguistic Inquiry 30: 1-25.

Broselow, Ellen. 1982. On the interaction of stress and epenthesis. Glossa 16: 115-132.

Browman, Catherine D. 1978. Tip of the tongue and slip of the ear: implications for language processing. UCLA Working Papers in Phonetics 42. Los Angeles: University of California, Los Angeles.

Brown, Roger, and David McNeill. 1966. The 'tip of the tongue' phenomenon. Journal of Verbal Learning and Verbal Behavior 5: 325-337.

Bruening, Benjamin, Yoonjung Kang, and Martha McGinnis, eds. 1997. PF: Papers at the Interface. MIT Working Papers in Linguistics 30. Cambridge, Mass.: MIT.

Buckley, Eugene. 1998. Alignment in Manam stress. Linguistic Inquiry 29: 475-496.

Buller, Barbara,' Ernest Buller, and Daniel L. Everett. 1993. Stress placement, syllable structure, and minimality in Banawá. IJAL 59: 280-93.

Bunn, Gordon, and Ruth Bunn. 1970. Golin phonology. Pacific Linguistics A-23: 1-7.

Burzio, Luigi. 1994. Principles of English Stress. Cambridge: Cambridge University Press. . 1997. Italian participial morphology and correspondence theory. In Geert Booij, Angela Ralli, and Sergio Scalise, eds., Proceedings of the First Mediterranean Conference of Morphology. University of Patras, 42-53.

Casali, Roderic F. 1996. Resolving Hiatus. Doctoral dissertation, UCLA.

- 1997. Vowel elision in hiatus contexts: Which vowel goes? Language 73: 493-533.

Catford, J.C. 1977. Fundamental Problems in Phonetics. Edinburgh: Edinburgh University Press.

Chierchia, Gennaro. 1982. An autosegmental theory of raddoppiamento. In James Pustejovsky and Peter Sells, eds., Proceedings of NELS 12. Amherst, Mass.: GLSA, 49-62.

Cho, Taehong, and Patricia Keating. 2001. Articulatory strengthening at the onset of prosodic domains in Korean. Journal of Phonetics 28: 155-190.

Chomsky, Noam. 1970. Remarks on nominalization. In Roderick A. Jacobs and Peter S. Rosenbaum, eds., Readings in English Transformational Grammar. Waltham, Mass.: Ginn, 184-221.

Chomsky, Noam, and Morris Halle. 1968. The Sound Pattern of English. New York: Harper \& Row.

Chung, Sandra. 1983. Transderivational relationships in Chamorro phonology. Language 59: 35-66.

Clements, George N. 1990. The role of the sonority cycle in core syllabification. In J. Kingston and M. Beckman, eds., 283-333. 
Clements, George N., and Samuel Jay Keyser. 1983. CV Phonology: A Generative Theory of the Syllable. Cambridge, Mass.: MIT Press.

Cohn, Abigail C. 1989. Stress in Indonesian and bracketing paradoxes. Natural Language and Linguistic Theory 7: 167-216.

- 1993. The initial dactyl effect in Indonesian and the status of degenerate feet. Linguistic Inquiry 24: 372-381.

Cohn, Abigail C., and John J. McCarthy. 1998. Alignment and parallelism in Indonesian phonology. In Niken Adisasmito-Smith, Bill Ham, and Lisa Lavoie, eds., Working Papers of the Cornell Phonetics Laboratory 12: 53-137. ROA \#25 [http://roa.rutgers.edu, 1994].

Cole, Jennifer, and Charles W. Kisseberth. 1994. An optimal domains theory of harmony. Studies in the Linguistic Sciences 24: 101-14. ROA \#22 [http://roa.rutgers.edu].

- 1995. Restricting multi-level constraint evaluation: Opaque rule interaction in Yawelmani vowel harmony. In K. Suzuki and D. Elzinga, eds., 18-38.

Cole, Ronald A. 1973. Listening for mispronunciations: A measure of what we hear during speech. Perception and Psychophysics 13: 153-156.

Cole, Ronald A., and Jola Jakimik. 1976. Factors affecting the detection of mispronunciations. Journal of the Acoustical Society of America 60 (supplement): M9.

- 1980. How are syllables used to recognize words? Journal of the Acoustical Society of America 67: 965-970.

Conathan, Lisa, Jeff Good, Darya Kavitskaya, Alyssa Wulf, and Alan Yu, eds. 2000. Proceedings of BLS 26. Berkeley, Cal.: Berkeley Linguistics Society.

Cook, Eung-Do. 1972. Stress and related rules in Tahltan. International Journal of American Linguistics 38: 231-33.

Crosswhite, Katherine M. 1999a. Segmental vs. prosodic correspondence in Chamorro. Phonology 15: 3.

- 1999b. Vowel Reduction in Optimality Theory. Doctoral dissertation, University of California, Los Angeles.

Crowhurst, Megan. 1992. Minimality and Foot Structure in Metrical Phonology and Prosodic Morphology. Doctoral dissertation, University of Arizona.

Crowhurst, Megan, and Mark Hewitt. 1997. Boolean operations and constraint interactions in Optimality Theory. Ms., University of North Carolina, Chapel Hill and Brandeis University. ROA \# 229 [http://roa.rutgers.edu].

Cutler, Anne. 1976. Phoneme monitoring reaction time as a function of preceding intonation contour. Perception and Psychophysics 20: 55-60.

-1986. Forbear is a homophone: Lexical prosody does not constrain lexical access. Language and Speech 29: 201-220.

Cutler, Anne, and Sally Butterfield. 1992. Rhythmic cues to speech perception: Evidence from juncture misperception. Journal of Memory and Language 31: 218-236.

Cutler, Anne, and Carter, D.M. 1987. The predominance of strong initial syllables in the English vocabulary. Computer Speech and Language 2: 133-142.

Cutler, Anne, and Hsuan-Chih Chen. 1997. Lexical tone in Cantonese spoken-word processing. Perception and Psychophysics 59: 165-179. 
Cutler, Anne, and Charles Clifton. 1984. The use of prosodic information in word recognition. In H. Bouma and D.G. Bowhuis, eds., 183-196.

Cutler, Anne, and Wilma van Donselaar. 2001. Voornaam is not (really) a homophone: Lexical prosody and lexical access in Dutch. Language and Speech 44: 171-195.

Cutler, Anne, Jacques Mehler, Dennis Norris, and Juan Segui. 1986. The syllable's differing role in the segmentation of French and English. Journal of Memory and Language 25: 385-400.

1987. Phoneme identification and the lexicon. Cognitive Psychology 19: 141-177.

Cutler, Anne, and Dennis Norris. 1988. The role of strong syllables in segmentation for lexical access. Journal of Experimental Psychology: Human Perception and Performance 14: 113-121.

Cutler, Anne, and Takashi Otake. 1999. Pitch accent in spoken-word recognition in Japanese. Journal of the Acoustical Society of America 105: 1877-1888. [Erratum: Journal of the Acoustical Society of America 106: 1191.]

Davis, Stuart. 1988. Syllable onsets as a factor in stress rules. Phonology 5: 1-19.

Davis, Stuart, and Michael Hammond. 1995. On the status of onglides in American English. Phonology 12: 159-182.

Davis, Stuart, Linda Manganaro, and Donna Jo Napoli. 1987. Stress on second conjugation infinitives in Italian. Italica 64: 477-498.

de Lacy, Paul. 1997. Prosodic Categorisation. Masters thesis, University of Auckland. ROA \#236 [http://roa.rutgers.edu]. - 1999. Tone and prominence. Ms., University of Massachusetts, Amherst. ROA $\# 333$ [http://roa.rutgers.edu].

- 2000. Prosodic markedness in prominent positions. Ms., University of Massachusetts, Amherst.

- 2001. Markedness in prominent positions. In Ora Matushansky, Albert Costa, Javier Martin-Gonzalez, Lance Nathan, and Adam Szczegielniak, eds., Proceedings of HUMIT 2000. Cambridge, Mass.: MITWPL 40. ROA \#432 [http://roa.rutgers.edu].

Delgutte, Bertrand. 1997. Auditory neural processing of speech. In William J. Hardcastle and John Laver, eds., The Handbook of Phonetic Sciences. Oxford: Blackwell, 507-538.

Dell, François, and Mohamed Elmedlaoui. 1985. Syllabic consonants and syllabification in Imdlawn Tashlhiyt Berber. Journal of African Languages and Linguistics 7: 105-130.

- 1988. Syllabic consonants in Berber: Some new evidence. Journal of African Languages and Linguistics 10: 1-17.

1992. Quantitative transfer in the nonconcatenative morphology of Imdlawn Tashlhiyt Berber. Journal of Afroasiatic Languages 3: 89-125.

Devine, A.M., and Laurence D. Stephens. 1994. The Prosody of Greek Speech. New York: Oxford University Press.

Dilley, Laura, Stefanie Shattuck-Hufnagel, and Mari Ostendorf. 1996. Glottalization of word-initial vowels as a function of prosodic structure. Journal of Phonetics 24: 423-444.

Dixon, R.M.W. 1977. A Grammar of Yidif. Cambridge: Cambridge University Press. 1980. The Languages of Australia. Cambridge: Cambridge University Press. 
1991. Mbabaram. In R.M.W. Dixon and Barry J. Blake, eds, Handbook of Australian Languages, vol. 4. Melbourne: Oxford University Press, 348-402.

Downing, Laura J. 1998. On the prosodic misalignment of onsetless syllables. Natural Language and Linguistic Theory 16: 1-52.

Dresher, B. Elan, and Harry van der Hulst. 1998. Head-dependent asymmetries in phonology. Phonology 15: 317-352.

Einarsson, Stefan. 1949. Icelandic: Grammar, Texts, Glossary. Baltimore, Md.: The Johns Hopkins Press.

Eisner, Jason. 1997. What constraints should OT allow? Paper presented at the LSA Annual Meeting; Chicago, January 4. ROA \#204 [http://roa.rutgers.edu].

Espy-Wilson, Carol Y. 1992. Acoustic measures for linguistic features distinguishing the semivowels /w j r $1 /$ in American English. Journal of the Acoustical Society of America 92: 736-757.

Everett, Daniel L. 1988. On metrical constituent structure in Pirahã phonology. Natural Language and Linguistic Theory 6: 207-246.

-1995. Quantity, sonority, and alignment constraints in Suruwahá and Banawá prosody. Ms., University of Pittsburgh. [Revised version available as ROA \#121, http://roa.rutgers.edu.]

Everett, Daniel L., and Keren Everett. 1984a. On the relevance of syllable onsets to stress placement. Linguistic Inquiry 15: 705-711.

1984b. Syllable onsets and stress placement in Pirahã. In Mark Cobler, Susannah MacKaye, and Michael T. Wescoat, eds., Proceedings of WCCFL 3. Stanford, Cal.: Stanford Linguistics Association, 105-116.

Feldman, Laurie B., and Jasmina Moskovljević. 1987. Repetition priming is not purely episodic in origin. Journal of Experimental Psychology: Learning, Memory, and Cognition 13: 573-581.

Fikkert, Paula. 1994. On the Acquisition of Prosodic Structure. Doctoral dissertation, University of Leiden. Leiden: Holland Institute of Generative Linguistics.

Flemming, Edward. 1995. Auditory Representations in Phonology. Doctoral dissertation, UCLA.

Forster, Kenneth I. 1976. Accessing the mental lexicon. In R.J. Wales and Edward Walker, eds., New Approaches to Language Mechanisms: A Collection of Psycholinguistic Studies. Amsterdam: North-Holland, 257-287.

—. 1990. Lexical processing. In D.N. Osherson and H. Lasnik, eds., 96-131.

Fougeron, Cécile. 1998. Variations articulatoires en début de constituants prosodiques de différents niveaux en français. Doctoral dissertation, Université Paris III.

Fougeron, Cécile, and Patricia A. Keating. 1997. Articulatory strengthening at edges of prosodic domains. Journal of the Acoustic Society of America 101: 3728-3740.

Fowler, Carol A., Shirley E. Napps, and Laurie Feldman. 1985. Relations among regular and irregular morphologically related words in the lexicon as revealed by repetition priming. Memory and Cognition 13: 241-255.

Frazier, Lyn. 1987. Structure in auditory word recognition. Cognition 25: 157-187. 
Fukazawa, Haruka, Mafuyu Kitahara, and Mitsuhiko Ota. 1998. Lexical stratification and ranking invariance in constraint-based grammars. In M. Gruber, D. Higgins, K. Olson, and T. Wysocki, eds., 47-62. ROA \#267 [http://roa.rutgers.edu].

Fukazawa, Haruka, and Linda Lombardi. 2000. To be simple or not to be: Constraints in Optimality Theory. Handout from presentation at the First Tokyo Conference on Psycholinguistics; Tokyo, March 25.

Furui, Sadaoki. 1986. On the role of spectral transition for speech perception. Journal of the Acoustical Society of America 80: 1016-1025.

Gahl, Susanne. 1996. Syllable onsets as a factor in stress rules: The case of Mathimathi revisited. Phonology 13: 329-344.

Garrett, Merrill F. 1990. Sentence processing. In D.N. Osherson and H. Lasnik, eds., 133-175.

Geisler, C. Daniel. 1998. From Sound to Synapse: Physiology of the Mammalian Ear. New York: Oxford University Press.

Gerfen, Chip. 1999. Phonology and Phonetics in Coatzospan Mixtec. Dordrecht: Kluwer Academic Publishers.

Goedemans, Rob. 1996. An optimality account of onset-sensitive phenomena in quantityinsensitive languages. The Linguistic Review 13: 33-48.

Goldinger, Steven D., Paul A. Luce, and David B. Pisoni. 1989. Priming lexical neighbors of spoken words: Effects of competition and inhibition. Journal of Memory and Language 28: 501-518.

Goldsmith, John. 1993. Harmonic phonology. In John Goldsmith, ed., The Last Phonological Rule: Reflections on Constraints and Derivations. Chicago: University of Chicago Press, 21-60.

- ed. 1995. The Handbook of Phonological Theory. Cambridge, Mass.: Blackwell.

Gordon, Matthew. 2000. Re-examining default-to-opposite stress. In L. Conathan, J. Good, D. Kavitskaya, A. Wulf, and A. Yu, eds.

Gow, David W., and Peter C. Gordon. 1993. Coming to terms with stress: Effects of stress location in sentence processing. Journal of Psycholinguistic Research 22: 545-578.

Green, Thomas. 1995. The stress window in Pirahã: A reanalysis of rhythm in Optimality Theory. Ms., MIT. ROA \#45 [http://roa.rutgers.edu].

Greenberg, Joseph H. 1941. Some problems in Hausa phonology. Language 17: 316-323.

Gregores, Emma, and Jorge A. Suarez. 1967. A Description of Colloquial Guarani. The Hague: Mouton.

Gruber, M. Catherine, Derrick Higgins, Kenneth S. Olson, and Tamra Wysocki, eds. 1998. CLS 34, Part 2: Papers from the Panels. Chicago: CLS.

Gussenhoven, Carlos. 2000. Vowel duration, syllable quantity and stress in Dutch. Ms., University of Nijmegen. ROA \#381 [http://roa.rutgers.edu].

Halle, Morris. 1973. Prolegomena to a theory of word formation. Linguistic Inquiry 4: 3-16. Hammond, Michael. 1997. Vowel quantity and syllabification in English. Language 73: $1-17$.

Handke, Jürgen. 1995. The Structure of the Lexicon: Human versus Machine. Natural Language Processing 5. Berlin: Mouton de Gruyter. 
Harris, James W. 1983. Syllable Structure and Stress in Spanish: A Nonlinear Analysis. Cambridge, Mass.: MIT Press.

Harris, James W., and Ellen M. Kaisse. 1999. Palatal vowels, glides and obstruents in Argentinean Spanish. Phonology 16: 117-190.

Hawkins, John A., and Anne Cutler. 1988. Psycholinguistic factors in morphological asymmetry. In John A. Hawkins, ed., Explaining Language Universals. Oxford: Blackwell, 280-317.

Hayes, Bruce. 1981. A Metrical Theory of Stress Rules. Bloomington, Ind.: Indiana University Linguistics Club.

- 1989. Compensatory lengthening in moraic phonology. Linguistic Inquiry 20: 253-306.

-1995. Metrical Stress Theory. Chicago: University of Chicago Press.

- 1999a. Phonetically driven phonology: The role of Optimality Theory and inductive grounding. In Michael Darnell, Edith A. Moravcsik, Frederick Newmeyer, Michael Noonan, and Kathleen M. Wheatley, eds., Formalism and Functionalism in Linguistics, vol. I. Amsterdam: Benjamins, 243-85. ROA \#158 [http://roa.rutgers.edu, 1996].

- 1999b. Phonological acquisition in Optimality Theory: The early stages. To appear in a volume edited by René Kager and Wim Zonneveld. ROA \#327 [http://roa.rutgers.edu].

Henderson, Leslie. 1985. Toward a psychology of morphemes. In Andrew W. Ellis, ed., Progress in the Psychology of Language, vol. 1. Hillsdale, N.J.: Lawrence Erlbaum Associates, 15-72.

Hercus, Luise. 1969. The Languages of Victoria: A Late Survey. Canberra: Australian Institute of Aboriginal Studies.

Hill, Jane, and Kenneth Hill. 1968. Stress in the Cupan (Uto-Aztecan) languages. International Journal of American Linguistics 34: 233-241.

Hollenbach, Barbara. 1977. Phonetic vs. phonemic correspondence in two Trique dialects. In William R. Merrifield, ed., Studies in Otomanguean Phonology. Arlington, Tex.: Summer Institute of Linguistics and the University of Texas at Arlington, 35-67.

Holt, David Eric. 1997. The Role of the Listener in the Historical Phonology of Spanish and Portuguese: An Optimality-Theoretic Account. Doctoral dissertation, Georgetown University.

Hooper, Joan Bybee. 1976. An Introduction to Natural Generative Phonology. New York: Academic Press.

Hualde, José Ignacio. 1989. Silabeo y estructura morfémica en español. Hispania 72: $475-493$.

Hulst, Harry van der. 1984. Syllable Structure and Stress in Dutch. Dordrecht: Foris.

1985. Ambisyllabicity in Dutch. In H. Bennis and F. Beukema, eds., Linguistics in the Netherlands 1985. Dordrecht: Foris, 57-66.

Hulst, Harry van der, René Kager, and Wim Zonneveld, eds. 1999. The Prosody-Morphology Interface. Cambridge: Cambridge University Press.

Hyman, Larry M. 1977. On the nature of linguistic stress. In L.M. Hyman, ed., 37-82.

, ed. 1977. Studies in Stress and Accent. Southern California Occasional Papers in Linguistics 4. Los Angeles: University of Southern California. 
1985. A Theory of Phonological Weight. Dordrecht: Foris. 255-273. 1988 . Underspecification and vowel height transfer in Esimbi. Phonology 5:

1998. Positional prominence and the 'prosodic trough' in Yaka. Phonology 15: 41-75. ROA \#225 [http://roa.rutgers.edu, 1997].

Inkelas, Sharon, C. Orhan Orgun, and Cheryl Zoll. 1997. The implications of lexical exceptions for the nature of grammar. In I. Roca, ed., 393-418.

Inkelas, Sharon, and Draga Zec. 1988. Serbo-Croatian pitch accent: The interaction of tone, stress, and intonation. Language 64: 227-248.

Itô, Junko. 1986. Syllable Theory in Prosodic Phonology. Doctoral dissertation, University of Massachusetts, Amherst. [New York: Garland, 1988.]

- 1989. A prosodic theory of epenthesis. Natural Language and Linguistic Theory 7: 217-259.

Itô, Junko, Yoshihisa Kitagawa, and Armin Mester. 1996. Prosodic faithfulness and correspondence: Evidence from a Japanese argot. Journal of East Asian Linguistics 5: 217-294. ROA \#146 [http://roa.rutgers.edu].

Itô, Junko, and Armin Mester. 1995. The core-periphery structure of the lexicon and constraints on reranking. In J. Beckman, L. Walsh Dickey, and S. Urbanczyk, eds., 181-209.

Jakobson, Roman. 1962. Selected Writings, vol. 1: Phonological Studies. The Hague: Mouton.

Jarvella, Robert J., and Guust Meijers. 1983. Recognizing morphemes in spoken words: Some evidence for a stem-organized mental lexicon. In Giovanni B. Flores d'Arcais and Robert J. Jarvella, eds., The Process of Language Understanding. New York: John Wiley and Sons, 81-113.

Jespersen, Otto. 1904. Lehrbuch der Phonetik. Leipzig: B.G. Teubner.

Jiang-King, Ping. 1996. An Optimality Account of Tone-Vowel Interaction in Northern Min. Doctoral dissertation, University of British Columbia. [Munich: Lincom Europa, 1999.] ROA \#266 [http://roa.rutgers.edu].

Jun, Jongho. 1995. Perceptual and Articulatory Factors in Place Assimilation: An Optimality Theoretic Approach. Doctoral dissertation, UCLA.

Jun, Sun-Ah. 1993. The Phonetics and Phonology of Korean Prosody. Doctoral dissertation, Ohio State University.

Jusczyk, Peter W., Angela D. Friederici, Jeanine M.I. Wessels, Vigdis Y. Svenkerud, and Ann Marie Jusczyk. 1993. Infants' sensitivity to the sound patterns of native language words. Journal of Memory and Language 32: 402-420.

Jusczyk, Peter W., Derek M. Houston, and Mary Newsome. 1999. The beginnings of word segmentation in English-learning infants. Cognitive Psychology 39: 159-207.

Kager, René. 1990. Dutch schwa in moraic phonology. CLS 26, vol. 2: Parasession on the Syllable in Phonetics and Phonology. Chicago: Chicago Linguistic Society, 241-255.

1999. Optimality Theory. Cambridge: Cambridge University Press.

Kahn, Daniel. 1976. Syllable-Based Generalizations in English Phonology. Doctoral dissertation, MIT. [New York: Garland, 1980.] 
Kang, Yoonjung. 1997. Tone in Sukuma. In B. Breuning, Y. Kang, and M. McGinnis, eds., 49-95.

Katz, Leonard, Suzanne Boyce, Louis Goldstein, and Georgije Lukatela. 1987. Grammatical information effects in auditory word recognition. Cognition 25: 235-263.

Kaye, Jonathan D., and Jean Lowenstamm. 1984. De la syllabicité. In François Dell, Daniel Hirst, and Jean-Roger Vergnaud, eds., Forme sonore du langage. Paris: Hermann, 123-159.

Keating, Patricia. 1983. Comments on the jaw and syllable structure. Journal of Phonetics 11: 401-406.

Keating, Patricia, Taehong Cho, Cécile Fougeron, and Chai-Shune Hsu. 1998. Domaininitial articulatory strengthening in four languages. [To appear in Papers in Laboratory Phonology VI.]

Keating, Patricia, Richard Wright, and Jie Zhang. 2001. Word-level asymmetries in consonant articulation. Ms., UCLA.

Keer, Edward W. 1999. Geminates, the OCP and the Nature of CON. Doctoral dissertation, Rutgers University. ROA \#350 [http://roa.rutgers.edu].

Kenstowicz, Michael. 1994. Sonority-driven stress. Ms., MIT. ROA \#33 [http://roa.rutgers.edu].

Kenstowicz, Michael, and Charles W. Kisseberth. 1979. Generative Phonology. Orlando, Fla.: Academic Press.

Kingston, John C. 1985. The Phonetics and Phonology of the Timing of Oral and Glottal Events. Doctoral dissertation, University of California, Berkeley.

. 1990. Articulatory binding. In J. Kingston and M. Beckman, eds., 406-434.

Kingston, John C., and Mary E. Beckman, eds. 1990. Papers in Laboratory Phonology I: Between the Grammar and Physics of Speech. Cambridge: Cambridge University Press.

Kisseberth, Charles W. 1969. On the abstractness of phonology: The evidence from Yawelmani. Papers in Linguistics 1: 248-282.

Kluender, Keith R., Andrew J. Lotto, and Rick L. Jenison. 1995. Perception of voicing for syllable-initial stops at different intensities: Does synchrony capture signal voiceless stop consonants? Journal of the Acoustical Society of America 97: 2552-2567.

Krause, Scott R. 1979. Topics in Chukchee Phonology and Morphology. Doctoral dissertation, University of Illinois, Urbana-Champaign.

Kuroda, Shige-Yuki. 1967. Yawelmani Phonology. Cambridge, Mass.: MIT Press.

Lahiri, Aditi, and B. Elan Dresher. 1999. Open syllable lengthening in West Germanic. Language 75: 678-719.

Landman, Meredith. 1999. Morphological contiguity. Ms., University of Massachusetts, Amherst.

Langdon, Margaret. 1975. Boundaries and lenition in Yuman languages. International Journal of American Linguistics 41: 218-33.

1977. Stress, length, and pitch in Yuman languages. In L.M. Hyman, ed., 239-259.

Lavoie, Lisa M. 2000. Phonological Patterns and Phonetic Manifestations of Consonant Weakening. Doctoral dissertation, Cornell University.

Lehiste, Ilse. 1970. Suprasegmentals. Cambridge, Mass.: MIT Press. 
Liberman, Mark. 1975. The Intonational System of English. Doctoral dissertation, MIT. [New York: Garland, 1979.]

Lombardi, Linda. 1991. Laryngeal Features and Laryngeal Neutralization. Doctoral dissertation, University of Massachusetts, Amherst. [New York: Garland, 1994.]

- 1999. Positional faithfulness and voicing assimilation in Optimality Theory. Natural Language and Linguistic Theory 17: 267-302.

- 2001. Why Place and Voice are different: constraint-specific alternations in Optimality Theory. In Linda Lombardi, ed., Segmental Phonology in Optimality Theory: Constraints and Representations. Cambridge: Cambridge University Press, 13-45.

Luce, Paul A. 1986. Neighborhoods of Words in the Mental Lexicon. Doctoral dissertation, Indiana University.

Luce, Paul A., and Michael S. Cluff. 1998. Delayed commitment in spoken-word recognition: Evidence from cross-modal priming. Perception and Psychophysics 60: 484-490.

Luce, Paul A., and David B. Pisoni. 1998. Recognizing spoken words: The neighborhood activation model. Ear and Hearing 19: 1-36.

Luce, Paul A., David B. Pisoni, and Steven D. Goldinger. 1990. Similarity neighborhoods of spoken words. In G.T.M. Altmann, ed., 122-147.

Lukatela, Georgije, Claudia Carello, and Michael T. Turvey. 1987. Lexical representation of regular and irregular inflected nouns. Language and Cognitive Processes 2: 1-17.

Lukatela, Georgije, B. Gligorijevic, A. Kostić, and Michael T. Turvey. 1980. Representation of inflected nouns in the internal lexicon. Memory and Cognition 8: 415-423.

Lynch, J. 1983. On the Kuman 'liquids'. Languages and Linguistics in Melanesia 14: 98-112.

Manelis, Leon, and David A. Tharp. 1977. The processing of affixed words. Memory and Cognition 5: 690-695:

Marslen-Wilson, William D. 1975. Sentence perception as an interactive parallel process. Science 189: 226-8.

- 1984. Function and process in spoken-word recognition: A tutorial review. In $\mathrm{H}$. Bouma and D.G. Bowhuis, eds., 125-150.

- 1987. Functional parallelism in spoken word-recognition. Cognition 25: 71-102.

Marslen-Wilson, William D., Colin M. Brown, and Pienie Zwitserlood. 1989. Spoken wordrecognition: Early activation of multiple semantic codes. Ms., Max-Planck-Institut, Nijmegen.

Marslen-Wilson, William D., and Alan Welsh. 1978. Processing interactions and lexical access during word recognition in continuous speech. Cognitive Psychology 10: 29-63.

Marslen-Wilson, William D., and Pienie Zwitserlood. 1989. Accessing spoken words: The importance of word onsets. Journal of Experimental Psychology: Human Perception and Performance 15: 576-585.

Maryott, Kenneth R. 1961. The phonology and morphophonemics of Tabukang Sangir. Philippine Social Sciences and Humanities Review 26: 111-126.

Mattys, Sven, and Arthur G. Samuel. 2000. Implications of stress-pattern differences in spoken-word recognition. Journal of Memory and Language 42: 571-596. 
McArthur, Harry, and Lucille McArthur. 1956. Aguacatec (Mayan) phonemes within the stress group. International Journal of American Linguistics 22: 72-76.

McCarthy, John J. 1998. Morpheme structure constraints and paradigm occultation. In M. Gruber, D. Higgins, K. Olson, and T. Wysocki, eds., 123-150.

. 1999. Sympathy and phonological opacity. Phonology 16: 331-399.

2000a. Faithfulness and prosodic circumscription. In Joost Dekkers, Frank van der Leeuw and Jeroen van de Weijer, eds., Optimality Theory: Syntax, Phonology, and Acquisition. Oxford: Oxford University Press, 151-189. ROA \#201 [http://roa.rutgers.edu].

2000b. The prosody of phase in Rotuman. Natural Language and Linguistic Theory 18: 147-197. ROA \#1 10 [http://roa.rutgers.edu].

2002. A Thematic Guide to Optimality Theory. New York: Cambridge University Press.

McCarthy, John J., and Alan S. Prince. 1986. Prosodic Morphology. Ms., University of Massachusetts, Amherst, and Brandeis University. [Available as Rutgers University Center for Cognitive Science Technical Report \#RuCCS-TR-32, 1996.]

- 1991. Prosodic minimality. Lecture presented at The Organization of Phonology conference, University of Illinois.

__ 1993a. Generalized alignment. Yearbook of Morphology 1993: 79-153.

- 1993b. Prosodic Morphology I: Constraint Interaction and Satisfaction. Rutgers University Center for Cognitive Science Technical Report RUCCS TR-3. [To appear, MIT Press.]

- 1994. The emergence of the unmarked: Optimality in prosodic morphology. In Mercé Gonzalez, ed., Proceedings of NELS 24. Amherst, Mass.: GLSA, 333-379.

. 1995. Faithfulness and reduplicative identity. In J. Beckman, L. Walsh Dickey, and S. Urbanczyk, eds., 250-384.

McClelland, James L., and Jeffrey L. Elman. 1986. The TRACE model of speech perception. Cognitive Psychology 18: 1-86.

Mehler, Jacques, Jean-Yves Dommergues, Uli Frauenfelder, and Juan Segui. 1981. The syllable's role in speech segmentation. Journal of Verbal Learning and Verbal Behavior 20: 298-305.

Meredith, Scott. 1990. Issues in the Phonology of Prominence. Doctoral dissertation, MIT. Michelson, Karin. 1988. A Comparative Study of Lake-Iroquoian Accent. Dordrecht: Kluwer.

Milner, Jean Claude. 1967. French truncation rule. Quarterly Progress Report of the Research Laboratory of Electronics (Massachusetts Institute of Technology) 86: 273-83.

- 1973. Arguments Linguistiques. Paris: Mame.

Minematsu, Nobuaki, and Hirose, Keikichi. 1995. Role of prosodic features in the human process of perceiving spoken words and sentences in Japanese. Journal of the Acoustical Society of Japan 16: 311-320.

Minkova, Donka. 1982. The environment for open syllable lengthening in Middle English. Folia Linguistica Historica 3: 29-58. 
Molen, Hugo van der, and John Morton. 1979. Remembering plurals: Unit of coding and form of coding during serial recall. Cognition 7: 35-47.

Morén, Bruce. 1999. Distinctiveness, Coercion and Sonority: A Unified Theory of Weight. Doctoral dissertation, University of Maryland, College Park. ROA \#346 [http://roa.rutgers.edu].

Muller, Jennifer S. 1999. A unified mora account of Chuukese. In S. Bird, A. Carnie, J. Haugen, and P. Norquest, eds., 393-405.

Murray, Robert W., and Theo Vennemann. 1983. Sound change and syllable structure in Germanic phonology. Language 59: 514-28.

Myers, Scott. 1998. Surface underspecification of tone in Chichewa. Phonology 15: 367-391.

Nakatani, Lloyd H., and Judith A. Schaffer. 1978. Hearing 'words' without words: Prosodic cues for word perception. Journal of the Acoustical Society of America 63: 234-245.

Nater, Hank F. 1989. Some comments on the phonology of Tahltan. International Journal of American Linguistics 55: 25-42.

Newell, Leonard E. 1956. Phonology of the Guhang Ifugao dialect. Philippine Journal of Science 85: 523-539.

Newman, Stanley. 1944. Yokuts Language of California. New York: Viking Fund.

Ní Chiosáin, Máire, and Jaye Padgett. 1997. Markedness, segment realization, and locality in spreading. Report no. LRC-97-01, Linguistics Research Center, Department of Linguistics, University of California, Santa Cruz.

Nooteboom, Sieb G. 1981. Lexical retrieval from fragments of spoken words: Beginnings vs endings. Journal of Phonetics 9: 407-424.

Norris, Dennis. 1994. Shortlist: A connectionist model of continuous speech recognition. Cognition 52: 189-234.

Norris, Dennis, James M. McQueen, and Anne Cutler. 1995. Competition and segmentation in spoken-word recognition. Journal of Experimental Psychology: Learning, Memory, and Cognition 21: 1209-1228.

Ohala, John J. 1992. The segment: Primitive or derived? In Gerard J. Docherty and D. Robert Ladd, eds., Papers in Laboratory Phonology II: Gesture, Segment, Prosody. Cambridge: Cambridge University Press, 166-183.

Osherson, Daniel N., and Howard Lasnik, eds. 1990. Language: An Invitation to Cognitive Science, vol. 1. Cambridge, Mass.: MIT Press.

Padgett, Jaye. 1995. Partial class theory and nasal place assimilation. In K. Suzuki and D. Elzinga, eds., 145-183.

Parker, Steve. 1994. Laryngeal codas in Chamicuro. International Journal of American Linguistics 60: 261-71.

- 2000. Non-optimal laryngeal onsets in Chamicuro. Ms., University of Massachusetts, Amherst. [Revised version published in Phonology 18 (2001): 361-386.]

-2002. Quanitifying the Sonority Hierarchy. Doctoral dissertation, University of Massachusetts, Amherst.

Pater, Joe. 1996. *NC. In Kiyomi Kusumoto, ed., Proceedings of NELS 26. Amherst, Mass.: GLSA, 227-239. 
1997. Minimal violation and phonological development. Language Acquisition 6: 201-253.

- 1999. Austronesian nasal substitution and other NC effects. In H. van der Hulst, R. Kager, and W. Zonneveld, eds., 310-343. ROA \#160 [http://roa.rutgers.edu, 1995].

Payne, David L. 1981. The Phonology and Morphology of Axininca Campa. Arlington, Tex.: Summer Institute of Linguistics and University of Texas at Arlington.

Pensalfini, Rob. 1998. The development of (apparently) onsetless syllabification: A constraint-based approach. In M. Gruber, D. Higgins, K. Olson, and T. Wysocki, eds., 167-177.

Pierrehumbert, Janet, and David Talkin. 1992. Lenition of $/ \mathrm{h} /$ and glottal stop. In Gerard J. Docherty and D. Robert Ladd, eds., Papers in Laboratory Phonology II: Gesture, Segment, Prosody. Cambridge: Cambridge University Press, 90-117.

Pitt, Mark A., and Arthur G. Samuel. 1995. Lexical and sublexical feedback in auditory word recognition. Cognitive Psychology 29: 149-188.

Poppe, Nikolaus. 1970. Mongolian Language Handbook. Washington, D.C.: Center for Applied Linguistics.

Prince, Alan S. 1980. A metrical theory for Estonian quantity. Linguistic Inquiry 11: 511-562.

- 1990. Quantitative consequences of rhythmic organization. CLS 26, vol. 2: Parasession on the Syllable in Phonetics and Phonology, 355-398.

Prince, Alan S., and Paul Smolensky. 1993. Optimality Theory: Constraint Interaction in Generative Grammar. Ms., Rutgers University and University of Colorado, Boulder.

Prince, Alan S., and Bruce Tesar. 1999. Learning phonotactic distributions. Ms., Rutgers University. ROA \#353 [http://roa.rutgers.edu]. [To appear in René Kager, Joe Pater, and Wim Zonneveld, eds., Fixing Priorites: Constraints in Phonological Acquisition. Cambridge University Press.]

Pulleyblank, Douglas. 1986. Tone in Lexical Phonology. Dordrecht: Reidel.

Radhakrishnan, R. 1981. The Nancowry Word: Phonology, Affixal Morphology and Roots of a Nicobarese Language. Carbondale, Ill.: Linguistic Research.

Ramsey, S. Robert. 1987. The Languages of China. Princeton: Princeton University Press. Reid, Agnieszka, and William D. Marslen-Wilson. 2000. Complexity and alternation in the Polish mental lexicon. Proceedings of Spoken Word Access Processes 2000.

Revithiadou, Anthi. 1999a. Headmost Accent Wins: Head Dominance and Prosodic Form in Lexical Accent Systems. The Hague: Holland Academic Graphics. ROA \#388 [http://roa.rutgers.edu].

- 1999b. Head-dependent asymmetries and the Root-Affix metaconstraint. Handout from poster presented at WCCFL 18, University of Arizona.

Rice, Keren D. 1987. Metrical structure in a tone language: The foot in Slave (Athapaksan). Proceedings of CLS 23, vol. 2: 239-252.

- 1992. On deriving sonority: A structural account of sonority relationships. Phonology 9: 61-99.

Rigler, Jakob. 1963. Juzhnonotranjski Govori: Akcent in glasoslov je govorov med snezhnikom in Slavnikom. Ljubljana: Slovenska Akademija Znanosti in Umetnosti. [Cited in Crosswhite 1999b.] 
Robinson, Lila W. 1975. Some phonological rules of Iowa-Oto (Siouan). Proceedings of the Mid-America Linguistics Conference. Lawrence, Kans.: University of Kansas Linguistics Department, 439-448.

Roca, Iggy, ed. 1997. Derivations and Constraints in Phonology. Oxford: Clarendon Press. Rosenberg, Sheldon, Paul J. Coyle, and Walter L. Porter. 1966. Recall of adverbs as a function of the frequency of their adjective roots. Journal of Verbal Learning and Verbal Behavior 5: 75-76.

Rosenthall, Sam. 1994. Vowel/Glide Alternation in a Theory of Constraint Interaction. Doctoral dissertation, University of Massachusetts, Amherst.

Rubach, Jerzy. 1993. The Lexical Phonology of Slovak. Oxford: Clarendon Press.

- 1998. A Slovak argument for the onset/rime distinction. Linguistic Inquiry 29: $168-179$.

Salzmann, Zdeněk. 1956. Arapaho I: Phonology. International Journal of American Linguistics 22: 49-56.

Scalise, Sergio. 1986. Generative Morphology. Dordrecht: Foris.

Schane, Sanford. 1968. French Phonology and Morphology. Cambridge, Mass.: MIT Press. Schreuder, Robert, and R. Harald Baayen. 1995. Modeling morphological processing. In Laurie Beth Feldman, ed., Morphological Aspects of Language Processing. Hillsdale, N.J.: Lawrence Erlbaum Associates, 131-154.

1997. How complex simplex words can be. Journal of Memory and Language 37: 118-139.

Segui, Juan, Emmanuel Dupoux, and Jacques Mehler. 1990. The role of the syllable in speech segmentation, phoneme identification, and lexical access. In G.T.M. Altmann, ed., 263-280.

Seidenberg, Mark S., Michael K. Tanenhaus, James M. Leiman, and Marie Bienkowski. 1982. Automatic access of the meanings of words in context: Some limitations of knowledge-based processing. Cognitive Psychology 14: 489-537.

Selkirk, Elisabeth O. 1974. French liaison and the $\bar{X}$ notation. Linguistic Inquiry 5: 573-589. - 1982. The syllable. In Harry van der Hulst and Norval Smith, eds., The Structure of Phonological Representations, vol. II. Dordrecht: Foris, 337-383.

. 1984. On the major class features and syllable theory. In Mark Aronoff and Richard T. Oehrle, eds., Language Sound Structure: Studies in Phonology Presented to Morris Halle by his Teacher and Students. Cambridge, Mass.: MIT Press, 107-113.

- 1986. On derived domains in sentence phonology. Phonology Yearbook 3: $371-405$.

- 1994. The constraint question for feature structure: A review of some answers. Course materials for Linguistics 730, Fall 1994; University of Massachusetts, Amherst.

- 1995. The prosodic structure of function words. In J. Beckman, L. Walsh Dickey, and S. Urbanczyk, eds., 439-469.

Shields, Joyce L., Astrid McHugh, and James G. Martin. 1974. Reaction time to phoneme targets as a function of rhythmic cues in continuous speech. Journal of Experimental Psychology 102: 250-255.

Shillcock, Richard. 1990. Lexical hypotheses in continuous speech. In G.T.M. Altmann, ed., 24-49. 
Sievers, Eduard. 1881. Grundzüge der Phonetik. Leipzig: Breitkopf and Härtel.

Silva, David J. 1992. The Phonetics and Phonology of Stop Lenition in Korean. Doctoral dissertation, Cornell University.

Silverman, Daniel. 1995. Phasing and Recoverability. Doctoral dissertation, UCLA. [New York: Garland, 1997.]

Silverman, Kim E.A., and Janet B. Pierrehumbert. 1990. The timing of prenuclear high accents in English. In J. Kingston and M. Beckman, eds., 72-106.

Smith, Jennifer L. 1998. Noun faithfulness and word stress in Tuyuca. In Jennifer Austin and Aaron Lawson, eds., Proceedings of ESCOL 97. Ithaca, N.Y.: CLC Publications, 180-191.

- 1999. Noun faithfulness and accent in Fukuoka Japanese. In S. Bird, A. Carnie, J. Haugen, and P. Norquest, eds., 519-531.

2000a. Positional faithfulness and learnability in Optimality Theory. In Rebecca Daly and Anastasia Riehl, eds., Proceedings of ESCOL 99. Ithaca, N.Y.: CLC Publications, 203-214.

- 2000b. Prominence, augmentation, and neutralization in phonology. In L. Conathan, J. Good, D. Kavitskaya, A. Wulf, and A. Yu, eds, 247-257.

- 2001. Lexical category and phonological contrast. In Robert Kirchner, Joe Pater, and Wolf Wikely, eds., Papers in Experimental and Theoretical Linguistics 6: Workshop on the Lexicon in Phonetics and Phonology. Edmonton, Alta.: University of Alberta, 61-72.

Smith, R.L. 1979. Adaptation, saturation, and physiological masking in single auditory-nerve fibers. Journal of the Acoustical Society of America 65: 166-78.

Smolensky, Paul. 1995. On the internal structure of the constraint component Con of UG. Handout from talk presented at UCLA. ROA \#86 [http://roa.rutgers.edu].

-1996. The Initial State and 'richness of the base' in Optimality Theory. Johns Hopkins University Department of Cognitive Science Technical Report \#JHU-CogSci96-4. ROA \#154 [http://roa.rutgers.edu].

- 1997. Constraint interaction in generative grammar II: Local Conjunction, or random rules in Universal Grammar. Handout from presentation at Hopkins Optimality Theory Workshop/Maryland Mayfest '97; Baltimore, Md..

Spring, Cari. 1990. Implications of Axininca Campa for Prosodic Morphology and Reduplication. Doctoral dissertation, University of Arizona, Tucson.

Sprouse, Ronald. 1997. A case for enriched inputs. Handout from presentation at Tri-Lateral Phonology Weekend (TREND) 1997. ROA \#193 [http://roa.rutgers.edu].

Stanners, Robert F., James J. Neiser, William P. Hernon, and Roger Hall. 1979. Memory representation for morphologically related words. Journal of Verbal Learning and Verbal Behavior 18: 399-412.

Stanners, Robert F., James J. Neiser, and Scott Painton. 1979. Memory representation for prefixed words. Journal of Verbal Learning and Verbal Behavior 18: 733-743.

Steriade, Donca. 1982. Greek Prosodies and the Nature of Syllabification. Doctoral dissertation, MIT.

- 1988. Reduplication and syllable transfer in Sanskrit and elsewhere. Phonology 5: 73-155. 
1993. Positional neutralization. Handout presented at NELS 23, University of Massachusetts, Amherst.

- 1995. Positional neutralization. Ms., University of California, Los Angeles. 1997. Phonetics in phonology: The case of laryngeal neutralization. Ms., UCLA.

- 1999a. Grammar of perceptibility effects: Direction in assimilation and cluster simplification. Handout from presentation at Phonology 2000; MIT.

. 1999b. Perceptual factors in place assimilation. Handout from presentation at the International Congress of Phonetic Science Satellite Session on Perception in Phonology; San Francisco.

Stevens, Kenneth N. 1971. The role of rapid spectrum changes in the production and perception of speech. In L.L. Hammerich, Roman Jakobson, and Eberhard Zwirner, eds., Form and Substance: Phonetic and Linguistic Papers Presented to Eli FischerJørensen. Copenhagen: Akademisk Forlag, 95-101.

1989. On the quantal nature of speech. Journal of Phonetics 17: 3-46.

Stevens, Kenneth N., and Samuel Jay Keyser. 1989. Primary features and their enhancement in consonants. Language 65: 81-106.

Stevick, Earl W. 1969. Tone in Bantu. International Journal of American Linguistics 35: 330-341.

Strehlow, Theodore G.H. 1942. Aranda phonetics. Oceania 12: 255-302.

Struijke, Caro. 1998. Reduplicant and output TETU in Kwakwala. In Haruka Fukazawa, Frieda Morelli, Caro Struijke, and Yi-Ching Su, eds., Papers in Phonology. University of Maryland Working Papers, vol. 7, 150-178.

2000. Reduplication, Feature Displacement, and Existential Faithfulness. Doctoral dissertation, University of Maryland, College Park.

Suzuki, Keiichiro. 1998. A Typological Investigation of Dissimilation. Doctoral dissertation, University of Arizona.

Suzuki, Keiichiro, and Dirk Elzinga, eds. 1995. Proceedings of South Western Optimality Theory Workshop 1995. Coyote Working Papers 5. Tucson, Ariz.: University of Arizona.

Swinney, David A. 1979. Lexical access during sentence comprehension: (Re)consideration of context effects. Journal of Verbal Learning and Verbal Behavior 18: 645-659.

Taft, Lori A. 1984. Prosodic Constraints and Lexical Parsing Strategies. Doctoral dissertation, University of Massachusetts, Amherst.

Taft, Marcus. 1979. Recognition of affixed words and the word frequency effect. Memory and Cognition 7: 263-272.

Taft, Marcus, and Kenneth I. Forster. 1975. Lexical storage and retrieval of prefixed words. Journal of Verbal Learning and Verbal Behavior 14: 638-47.

Taft, Marcus, Gail Hambly, and Sachiko Kinoshita. 1986. Visual and auditory recognition of prefixed words. Quarterly Journal of Experimental Psychology 38A: 351-366.

Teoh, Boon Seong. 1988. Aspects of Malay Phonology Revisited: A Non-Linear Approach. Doctoral dissertation, University of Illinois.

Trefry, D. 1969. A Comparative Study of Kuman and Pawaian. Pacific Linguistics B-13. Canberra: The Australian National University. 
Trubetzkoy, Nikolai Sergeevich. 1939. Principles of Phonology. Translated by Christiane A. M. Baltaxe. Berkeley, University of California Press, 1969.

Truckenbrodt, Hubert. 1999. On the relation between syntactic phrases and phonological phrases. Linguistic Inquiry 30: 219-255.

Tsukamoto, Akihisa. 1988. A Grammar of Niuafo'ou. Doctoral dissertation, Australian National University.

Tsygankin, D.B., and C.Z. Debaev. 1975. Očrk sravnitel'noj grammatiki mordovskix (mokšanskogo i erz'anskogo) literaturnix jazykov. Saransk.

Urbanczyk, Suzanne. 1996a. Morphological templates in reduplication. In Kiyomi Kusumoto, ed., Proceedings of NELS 26. Amherst, Mass.: GLSA, 425-440.

- 1996b. Patterns of Reduplication in Lushootseed. Doctoral dissertation, University of Massachusetts, Amherst.

- 1999a. A-templatic reduplication in Halq'eméylem'. In Kimary N. Shahin, Susan J. Blake, and Eun-Sook Kim, eds., Proceedings of WCCFL XVII. Stanford, Cal.: CSLI, 655-69.

. 1999b. Double reduplications in parallel. In H. van der Hulst, R. Kager, and W. Zonneveld, eds., 390-428.

Vennemann, Theo. 1972. On the theory of syllabic phonology. Linguistische Berichte 18: $1-18$.

- 1988. Preference Laws for Syllable Structure and the Explanation of Sound Change. New York: Mouton de Gruyter.

Vitevitch, Michael S., and Paul A. Luce. 1999. Probabilistic phonotactics and neighborhood activation in spoken word recognition. Journal of Memory and Language 40: 374-408.

Voegelin, Charles. 1935. Tübatulabal Grammar. University of California Publications in American Archeaology and Ethnology, vol. 34, no. 2. Berkeley, Cal.: University of California Press.

Vroomen, Jean, and Beatrice de Gelder. 1995. Metrical segmentation and lexical inhibition in spoken word recognition. Journal of Experimental Psychology: Human Perception and Performance 21: 98-108.

Walker, Rachel. 1996. Prominence-driven stress. Ms., University of California, Santa Cruz. ROA \#172 [http://roa.rutgers.edu].

- 1999. Esimbi vowel height shift: Implications for Faith and Markedness. Ms., University of Southern California. ROA \#336 [http://roa.rutgers.edu].

- To appear. Positional markedness in vowel harmony. Proceedings of the Fifth HIL Phonology Conference.

Walsh Dickey, Laura. 1996. Limiting-domains in lexical access: The processing of lexical prosody. In Michael Walsh Dickey and Susanne Tunstall, eds., Linguistics in the Laboratory. University of Massachusetts Occasional Papers 19. Amherst, Mass.: GLSA, 133-155.

- 1997. The Phonology of Liquids. Doctoral dissertation, University of Massachusetts, Amherst.

- In preparation. The processing of lexical prosody: Studies on Igbo and Japanese. Ms., Max-Planck-Institut and Northwestern University. 
Warner, Natasha L. 1998. The Role of Dynamic Cues in Speech Perception, Spoken Word Recognition, and Phonological Universals. Doctoral dissertation, University of California, Berkeley.

Westbury, John R., and Patricia A. Keating. 1986. On the naturalness of stop consonant voicing. Journal of Linguistics 22: 145-166.

Wetzels, W. Leo, and Joan Mascaró. 2001. The typology of voicing and devoicing. Language 77: 207-244.

Wilson, Colin. 2000. Targeted Constraints: An Approach to Contextual Neutralization in Optimality Theory. Doctoral dissertation, Johns Hopkins University.

- 2001. Consonant cluster neutralisation and targeted constraints. Phonology 18: 147-197.

Woo, Nancy. 1969. Prosody and Phonology. Doctoral dissertation, MIT.

Woodbury, Anthony. 1987. Meaningful phonological processes: A consideration of Central Alaskan Yupik prosody. Language 63: 685-740.

$\mathrm{Xu}, \mathrm{Yi}$. 1999. Effects of tone and focus on the formation and alignment of $f_{0}$ contours. Journal of Phonetics 27: 55-105.

Yallop, Colin. 1977. Alyawarra: An Aboriginal Language of Central Australia. Canberra: Australian Institute of Aboriginal Studies.

Yip, Moira. 1980. The Tonal Phonology of Chinese. Doctoral dissertation, MIT.

- 1995. Tone in East Asian languages. In J. Goldsmith, ed., 476-494. 2000. The complex interaction of tone and prominence. Handout from presentation at NELS 31, Georgetown University.

Zec, Draga. 1988. Sonority Constraints on Prosodic Structure. Doctoral dissertation, Stanford University. [New York: Garland, 1994.]

- 1995. Sonority constraints on syllable structure. Phonology 12: 85-129.

. 1999. Footed tones and tonal feet: Rhythmic constituency in a pitch-accent language. Phonology 16: 225-264.

Zhang, Jie. 2000. The phonetic basis for tonal melody mapping in Mende and elsewhere. In Roger Billerey and Brook Lillehaugen, eds., Proceedings of WCCFL 19. Somerville, Mass.: Cascadilla Press, 603-616.

Zoll, Cheryl. 1993. Directionless syllabification and ghosts in Yawelmani. Ms., University of California, Berkeley. ROA \#28 [http://roa.rutgers.edu].

-1996. Parsing below the Segment in a Constraint-Based Framework. Doctoral dissertation, University of California, Berkeley.

- 1997a. Conflicting directionality. Phonology 14: 263-286.

- 1997b. A note on multiple prominence and tone mapping. In B. Bruening, Y. Kang, and M. McGinnis, eds., 97-112.

- 1998. Positional asymmetries and licensing. Ms., MIT. ROA \#282 [http://roa.rutgers.edu].

Zwitserlood, Pienie. 1989. The locus of effects of sentential-semantic context in spokenword processing. Cognition 32: 25-64. 


\section{Index of Names}

Alderete, John, 14, 72, 188-190, 192, Boudelaa, Sami, 216-218 193, 195, 211, 218, 251, $253 \quad$ Boyce, Suzanne, 213

Altmann, Gerry T. M., 204

Anttila, Arto, 18, 269

Archangeli, Diana, 35, 37, 138

Aronoff, Mark, 211

Arvaniti, Amalia, 228

Baayen, R. Harald, 212, 217

Baković, Eric, 99

Barnes, Janet, 7, 189-193

Beckman, Jill N., 8, 14, 81, 86, 87, $89,90,92,95,96,147,149$, $150,188,193,210,211$, $232,233,245,247,248$, 251, 253, 255, 256, 273

Beckman, Mary E., 71

Benua, Laura, 31

Bienkowski, Marie, 205

Blevins, Juliette, 166, 167, 170

Bloch, Bernard, 56

Bloomfield, Leonard, 56

Boersma, Paul, 33, 37

Bolognesi, Roberto, 158-162, 174, $186,242-245$

Bond, Zinny S., 222

Booij, Geert, 117, 119-121

Borowsky, Toni, 51

Bradley, Dianne, 216, 217

Breen, Gavan, 122, 126

Broselow, Ellen, 51, 196

Browman, Catherine D., 206

Brown, Colin M., 208

Brown, Roger, 206, 220, 274

Buckley, Eugene, 125

Buller, Barbara, 126

Buller, Ernest, 126

Bunn, Gordon, 105, 107

Bunn, Ruth, 105, 107

Burani, Cristina, 212

Burzio, Luigi, 31

Butterfield, Sally, 221

Carello, Claudia, 213

Carter, D.M., 224

Casali, Roderic F., 14, 81, 86, 87, 147, 149, 188, 210, 232, $233,248,251,253,255$, 256, 273

Chen, Hsuan-Chih, 51, 227, 228, 230

Chierchia, Gennaro, 51

Cho, Taehong, 245, 246

Chomsky, Noam, 68, 72, 211

Chung, Sandra, 271 
Clements, George N., 56, 57, 62, 65, 176, 177

Clifton, Charles, 222, 223

Cluff, Michael S., 221

Cohn, Abigail C., 241

Cole, Jennifer, 138-140

Cole, Ronald A., 207, 208, 220, 222, 223

Cook, Eung-Do, 189

Coyle, Paul J., 212

Crosswhite, Katherine M., 109-111, 271

Crowhurst, Megan, 39, 40, 98, 125 , 196, 262

Cutler, Anne, 205, 210, 211, 220-230, 234, 236

Davis, Stuart, 96, 135, 136, 173, 175, 182

de Gelder, Beatrice, 221, 222, 224, 225

de Lacy, Paul, 54, 65, 70, 71, 96, 105, $107,127,128,158,271$

Debaev, C.Z., 113

Delgutte, Bertrand, 45, 60, 61

Dell, François, 58, 63

Devine, A.M., 57

Dijkstra, Ton, 212

Dilley, Laura, 245

Dixon, R.M.W., 72, 167, 168, 171, 172, 189

Dommergues, Jean-Yves, 210

Donselaar, Wilma van, 223

Downing, Laura J., 122, 124-126, 201

Dresher, B. Elan, 102, 265

Dupoux, Emmanuel, 210

Einarsson, Stefan, 57, 271

Eisner, Jason, 27, 29

Elman, Jeffrey L., 205, 210

Elmedlaoui, Mohamed, 58, 63

Espy-Wilson, Carol Y., 57
Everett, Daniel L., 6, 96, 126, 129-132, 134

Everett, Keren, 6, 96, 129, 130, 132, 134

Feldman, Laurie B., 214-216

Fikkert, Paula, 225

Flemming, Edward, 37

Forster, Kenneth I., 204, 205, 209, 216, 234

Fougeron, Cécile, 245, 246

Fowler, Carol A., 214, 215

Frauenfelder, Uli, 210

Fukazawa, Haruka, 18, 27, 178, 260

Furui, Sadaoki, 69

Gahl, Susanne, 136

Garrett, Merrill F., 204

Geisler, C. Daniel, 45

Gerfen, Chip, 271

Gligorijevic, B., 213

Goedemans, Rob, 125

Goldinger, Steven D., 234-236

Goldsmith, John, 138

Goldstein, Louis, 213

Gordon, Matthew, 99

Gordon, Peter C., 222

Gow, David W., 222

Green, Thomas, 133

Gregores, Emma, 151

Gussenhoven, Carlos, 50, 52

Hall, Roger, 213-215

Halle, Morris, 68, 72, 211

Hambly, Gail, 216

Hammond, Michael, 50, 59, 173, 175, 182

Handke, Jürgen, 204

Harris, James W., 130, 173, 175,

178-182

Hawkins, John A., 211

Hayes, Bruce, 4, 28, 33, 35, 36, 46, $54,71,72,84,91,96,98$, 


$$
\begin{aligned}
& 105,107,113,135,175, \\
& 255,270
\end{aligned}
$$

Henderson, Leslie, 211

Hercus, Luise, 136

Hernon, William P., 213-215

Hewitt, Mark, 39, 40, 98, 125, 262

Hill, Jane, 189

Hill, Kenneth, 189

Hirose, Keikichi, 228

Hollenbach, Barbara, 90

Holt, David Eric, 52

Hooper, Joan Bybee, 14, 65

Houston, Derek M., 225

Hsu, Chai-Shune, 245, 246

Hualde, José Ignacio, 175, 178

Hulst, Harry van der, 50, 265

Hyman, Larry M., 72, 135, 199, 225 , 248, 274

Inkelas, Sharon, 178

Itô, Junko, 16, 47, 51, 62, 178

Jakimik, Jola, 207, 208, 220, 222 , 223

Jakobson, Roman, 126

Jarvella, Robert J., 212, 214, 217

Jenison, Rick L., 70

Jespersen, Otto, 56, 58

Jiang-King, Ping, 70

Jun, Jongho, 14, 87

Jun, Sun-Ah, 245, 246

Jusczyk, Peter W., 225

Kager, René, 50, 76, 247

Kahn, Daniel, 62

Kaisse, Ellen M., 173, 175, 178-182

Kang, Yoonjung, 15, 71, 107, 274

Katz, Leonard, 213

Kaye, Jonathan D., 173, 175-178

Keating, Patricia A., 35, 56, 58, 245, 246

Keer, Edward W., 255
Kenstowicz, Michael, 49, 54, 58, 96, $99,110,113,138,139$

Keyser, Samuel Jay, 35, 43, 44, 57, $62,176,177$

Kingston, John C., 15, 57, 70, 75, 87

Kinoshita, Sachiko, 216

Kisseberth, Charles W., 6, 138-140

Kitagawa, Yoshihisa, 47

Kitahara, Mafuyu, 18, 178

Kluender, Keith R., 70

Kostić, A., 213

Krause, Scott R., 77, 189

Kuroda, Shige-Yuki, 6, 138

Ladd, D. Robert, 228

Lahiri, Aditi, 102

Landman, Meredith, 151, 154, 156, 239, 240

Langdon, Margaret, 189, 190

Lavoie, Lisa M., 56, 57, 245

Lehiste, Ilse, 71, 96

Leiman, James M., 205

Liberman, Mark, 72

Lombardi, Linda, 14, 15, 27, 68, 75, $87,251,257,260,271$

Lotto, Andrew J., 70

Lowenstamm, Jean, 173, 175-178

Luce, Paul A., 205, 221, 234-236

Lukatela, Georgije, 213, 214

Lynch, J., 166

Manelis, Leon, 217

Manganaro, Linda, 136

Marslen-Wilson, William D., 205, 207-210, 214, 216-218, 234, 235

Martin, James G., 222, 223

Maryott, Kenneth R., 151

Mascaró, Joan, 188

Mattys, Sven, 207, 220, 222

McArthur, Harry, 98-100

McArthur, Lucille, 98-100 
McCarthy, John J., 3, 13, 14, 16, 29-31, 39, 47, 51, 62, 63, 76, $100,111,112,118,119$, $121,123,125,138,139$, $152,153,175,188,189$, 193, 196, 200, 211, 241, $251,253,255,260$

McClelland, James L., 205, 210

McHugh, Astrid, 222, 223

McNeill, David, 206, 220, 274

McQueen, James M., 205, 219, 221, 224-226, 234, 236

Mehler, Jacques, 210, 221

Meijers, Guust, 212, 214, 217

Mennen, Ineke, 228

Meredith, Scott, 70

Mester, R. Armin, 47, 51, 178

Michelson, Karin, 6, 97, 101

Milner, Jean Claude, 177

Minematsu, Nobuaki, 228

Minkova, Donka, 102

Molen, Hugo van der, 212

Morén, Bruce, 58

Morton, John, 212

Moskovljević, Jasmina, 214, 216

Muller, Jennifer S., 197

Murray, Robert W., 97, 102

Myers, Scott, 70

Nakatani, Lloyd H., 220

Napoli, Donna Jo, 136

Napps, Shirley E., 214, 215

Nater, Hank F., 189

Neiser, James J., 213-215

Newell, Leonard E., 151, 154, 155, 239, 240

Newman, Stanley, 138

Newsome, Mary, 225

Ní Chiosáin, Máire, 37

Nooteboom, Sieb G., 10, 208, 232-234, 274
Norris, Dennis, 205, 210, 219-221, 224-226, 234, 236

Ohala, John J., 62

Orgun, C. Orhan, 178

Ostendorf, Mari, 245

Ota, Mitsuhiko, 18, 178

Otake, Takashi, 229

Padgett, Jaye, 14, 15, 37

Painton, Scott, 215

Parker, Steve, 55, 56, 68, 140-143, 271

Pater, Joe, 28, 37, 211, 225

Payne, David L., 68, 153

Pensalfini, Rob, 122, 126

Pierrehumbert, Janet, 56, 228, 245, 246

Pisoni, David B., 205, 234-236

Pitt, Mark A., 209

Poppe, Nikolaus, 165

Porter, Walter L., 212

Prince, Alan S., 3, 13, 14, 16, 17, 20, 27, 29-31, 39, 41, 44, 47, 49, $51-55,62-65,72,76,100$, $111,112,118,119,123-125$, $152,153,175,188,189$, 193, 196, 200, 211, 251, $253,255,260,270$

Pulleyblank, Douglas, 35, 37, 70

Radhakrishnan, R., 189

Ramsey, S. Robert, 165, 166

Reid, Agnieszka, 214

Revithiadou, Anthi, 218

Rice, Keren D., 105, 106, 265

Rigler, Jakob, 110, 111

Robinson, Lila W., 126

Rosenberg, Sheldon, 212

Rosenthall, Sam, 51, 117, 119, 120, 182,183

Rubach, Jerzy, 175, 178 
Salzmann, Zdeněk, 7, 19, 20, 151, 152, 239

Samuel, Arthur G., 207, 209, 220, 222

Scalise, Sergio, 211

Schaffer, Judith A., 220

Schane, Sanford, 176

Schreuder, Robert, 212, 217

Segui, Juan, 210, 221

Seidenberg, Mark S., 205

Selkirk, Elisabeth O., 15, 56, 58, 62, $176,251,253$

Shattuck-Hufnagel, Stefanie, 245

Shields, Joyce L., 222, 223

Shillcock, Richard, 236

Sievers, Eduard, 56

Silva, David J., 245

Silverman, Daniel, 62

Silverman, Kim E.A., 228

Smith, Jennifer L., 14, 189-192, 196, 255,270

Smith, R.L., 60

Smolensky, Paul, 3, 13, 16, 17, 20, 27, 29, 30, 41, 44, 49, 51-55, $62-65,72,124,253,260$, 270

Spring, Cari, 153

Sprouse, Ronald, 138, 139

Stanners, Robert F., 213-215

Stephens, Laurence D., 57

Steriade, Donca, 8, 14, 15, 37, 56, 62, $65,80,83,86,87,89,96$, $149,188,251,252,257$, 273, 274, 276

Stevens, Kenneth N., 35, 43, 44, 57, 62,69

Stevick, Earl W., 70

Strehlow, Theodore G.H., 96, 122, 124, 201

Struijke, Caro, 211, 255
Suarez, Jorge A., 151

Suzuki, Keiichiro, 30, 138

Swinney, David A., 205

Taft, Lori A., 10, 219, 221, 222, 224-226, 232-234, 237

Taft, Marcus, 216, 217

Talkin, David, 56, 245, 246

Tanenhaus, Michael K., 205

Teoh, Boon Seong, 271

Tesar, Bruce, 255, 270

Tharp, David A., 217

Trager, George L., 56

Trefry, D., 166, 167

Trubetzkoy, Nikolai Sergeevich, 13, $14,72,96,149,251,252$, 273, 274

Truckenbrodt, Hubert, 197

Tsukamoto, Akihisa, 127

Tsygankin, D.B., 113

Turvey, Michael T., 213

Urbanczyk, Suzanne, 188, 211

Vennemann, Theo, 14, 56, 58, 97, 102

Voegelin, Charles, 72

Vroomen, Jean, 221, 222, 224, 225

Walker, Rachel, 52, 54, 96, 98-100, 105, 107, 188, 200, 248, 264

Walsh Dickey, Laura, 165, 227-230

Warner, Natasha L., 62, 69

Welsh, Alan, 205, 207, 210, 234

Westbury, John R., 35

Wetzels, W. Leo, 188

Wilson, Colin, 37, 57, 205, 207-210, 214, 216-218, 234, 235, 257

Woo, Nancy, 50

Woodbury, Anthony, 72

Wright, Richard, 246

$\mathrm{Xu}, \mathrm{Yi}, 228$

Yallop, Colin, 124

Yip, Moira, 50, 71, 107, 274 
Zec, Draga, 57, 58, 65, 105, 108

Zhang, Jie, 14, 246

Zoll, Cheryl, 14, 18, 30, 51, 99, 107,

$178,251,253,256,259-261$,

264

Zwitserlood, Pienie, 205, 208-210 


\section{Index of Languages}

Aguacatec, 96-100, 103, 144

Alyawarra, 124

Arabic, 92, 216, 217

Aranda-see Arrernte, Western

Arapaho, 7, 18-20, 22, 43, 151-156, $187,198,239,241,248,271$

Arrernte, Western, 96, 103, 117, $122-126,134,136,144,172$, 201

Axininca Campa, 68, 153

Bakairi, 188

Bantu, 14, 248

Berber, Imdlawn Tashlhiyt, 57, 58, 63

Cahuilla, 199

Chamicuro, 43, 68, 69, 140-144, 271

Chichewa, 70

ChiMwi:ni, 15

Chinese, 50, 71

Cantonese, 227-230

Mandarin, 228

Taiwanese, 246

Chukchee, 77, 189

Chuukese, 197

Copala Trique, 90

Cupeño, 189, 190, 195

Czech, 199

Dhangar-Kurux, 90, 92, 248
Diegueño, 189, 190, 198

Doyayo, 90,92

Dutch, 18, 19, 22, 50, 52, 96, 103,

117, 119-123, 126, 144, 205, 208, 212-214, 217, 219,

221-226, 230

English, 15, 50, 52, 57, 59, 90, 114, $115,128,136,144,145$, $175,182,206,210,212$, 213, 215-217, 219-226, 228-230, 246

Esimbi, 248, 264

Finno-Ugric, 113, 199

French, 175-178, 181, 182, 185, 210, 221,246

German, 92

Germanic, West, 52, 96-98, 102, 103

Golin, 96, 103, 105, 107, 109, 144

Gonja, 57

Greek, 228

Guaraní, 90, 151

Guhang Ifugao, 151, 152, 154-156, $187,198,239-241,248$

Guugu Yimidhirr, 67, 157, 158, 165, $167-169,173,184,187,239$

Halq'eméylem', 188

Hausa, 151 
Icelandic, 57, 271

Igbo, 227-229

Indonesian, 241

Italian, 90, 136, 158, 159, 162, 212

Japanese, 228-230, 253-255, 258

Kobon, 58, 59

Korean, 174, 246

Kuman, 157, 158, 165-167, 169, 170, $173,184,187,198,239$

Madimadi-see Mathimathi

Malay, Kelantan, 271

Mathimathi, 136

Mbabaram, 59, 157, 158, 164, $171-173,187,189,198,239$

Mixtec, Coatzospan, 271

Mohawk, 6, 43, 96-98, 101-103, 144

Mongolian, 157, 158, 165-167, 173, $184,185,187,198,239$, 262,263

Mordwin, Mokshan, 58, 59, 96, 103, $109,113,114,144,145$

Nancowry, 189

Nawuri, 248

Niuafo'ou, 67, 96, 103, 117, 126-129, $134,144,271$

Pirahã, 6, 57-59, 68, 69, 96, 103, 117, $126,129-131,133-136,144$, 187

Pitta-Pitta, 67, 157, 158, 165, 167, $168,184,187,239$

Polish, 214, 225, 226

Proto-Lake-Iroquoian, 101

Quechua, 92, 253-255, 258

Cuzco, 92

Russian, 226, 251, 254, 256, 258, 261

Sanskrit, 65, 92, 188

Sardinian, Campidanian, 59, 66, 157-161, 164, 165, 169, 171, $174,181,186,187,198$, $239,242,243$
Iglesias, 174, 186

Sestu, 59, 66, 158-162, 164, 165, $171,174,186,187,239$, 242-245

Serbo-Croatian, 57, 96, 103, 105, $108,213,214,216$

Shilluk, 90, 92, 248

Shona, 90, 92, 248

Slave, Hare, 96, 103, 105, 106, 144

Slovak, 175, 178, 182

Slovene, 59, 96, 103, 109-111, 113-115, 144, 145, 271

Spanish, 130, 175, 178-182, 184, 185,225

Sukuma, 15, 71, 107

Tabukang Sangir, 151

Tahltan, 189

Tiriyó, 143

Trukese-see Chuukese

Tübatulabal, 72

Turkish, 92, 226

Tuyuca, 7, 189-191, 195, 198

!Xóõ, 90, 92, 248

Yawelmani-see Yokuts, Yawelmani Yidin ${ }^{\mathrm{y}}, 72$

Yokuts, Yawelmani, 6, 59, 137-139, 144, 145

Yowlumne-see Yokuts, Yawelmani

Yupik, Central Alaskan, 72

Zulu, 92 


\section{Index of Subjects}

augmentation-see constraints, augmentation; positional augmentation

CON (universal constraint set), 3-12, $24,25-42,43-48,51,70$, $72-74,78,81,82,84,85$, 87-89, 91-93, 116, 143, 221, $237,238,247,265,273,278$ constraint construction, 12, 28, 29, $33,37,42,91,148$ constraint filters-see under Schema/Filter model constraint schemas-see under

Schema/Filter model constraint set-see CON constraints

augmentation constraints-see constraints, augmentation COINCIDE constraints, $15,18,88$, $99,251,252,259-265,268$

faithfulness constraints-see under faithfulness markedness constraints-see under markedness positional (relativized) constraints, passim; see especially 4-8, 12, 15, 16-23,
25-29, 31-33, 37-43, 45, 50, $65,71,73-78,82,87-92,96$, $104,116,121,137,143-146$, 148, 149, 197-199, 218, 247, 251-259, 264, 272, 273-275

constraints, augmentation defined, 3, 8-12

HaveCPlace, 33, 49, 56, 68, 69, $78,85,140,144,148,198$, 199

HaVECPlaCe/Onset, 88, 140-143, 144

HAVESTRESS, 49, 71-73, 76, $78-80,85,143,144,188$, 198-201, 203

HAVESTRESS/Root, 7, 11, 71, 76, $78,85,148,188-199,202$, 203, 219, 227

HEAVYo, 12, 37, 47, 49-51, 70, $71,78,85,98,144,148$, 198, 199

HEAVYo/ó, 6, 12, 37, 47, 49-54, $78,88,97-105,116$, 129-134, 144 HTONE, 49, 54, 69-71, 78, 85, 144, 198, 199, 201, 203, 219 
HTONE/ó, 54, 69, 70, 78, 104, 105-109, 116, 144

ONSET, 4, 5, 16, 17, 19, 27, 32, 40, 41, 49, 50, 59-63, 66-68, $78,85,116-122,125,128$, $129,135,144,150-156,162$, $184,187,198,240,249$, 271, 276

ONSET/ó, 19, 25, 40, 41, 54, 78, $88,91,104,117-126,128$, $129,133,144,201,272$

ONSET $/ \sigma_{1}, 7,10,19,20,50,78$, $84,148,150-156,162,163$, 197-199, 202, 203, 238-241 [*ONSET/X], 4, 5, 32, 44, 49, 55, 58, 59-62, 64-69, 78-80, 85, $116,126,127,129,131$, $134,135,143,144,149$, 156-158, 164, 171, 181-184, 187, 188, 198, 199, 249, 276 [*ONSET/X]/ó, 6, 54, 78, 88, 91, 104, 117, 126-134, 144, 187, 271

[*ONSET/X]/o $1,10,67,78,84$, $148,150,156-175,181$, 184-187, 188, 197, 198, 202, 203, 238, 239, 245, 246

[*PEAK/X], 11, 44, 49, 50, 53, 54, 55-59, 64-66, 8-80, 85, $109,110,116,137,143-145$, 148, 198, 199, 277

[*PEAK/X]/ó, 50, 54, 78, 88, 104, 109-116, 144, 145, 148, 190, 266, 268, 277

[*PEAK/X]/V:, 6, 78, 88, 137-140, 144, 145

enhancement of prominence-see EVAL, 277 factorial typology-see under typology

faithfulness

faithfulness constraints, defined, 32

positional faithfulness, 15,36 , 87-92, 110, 147, 190, 192, 210, 233, 237, 247, 251-256, 258-260, 266-268, 270, 276 formal/functional interactions in phonology, 4, 5, 12, 23, 25, 26-38, 42-48, 66-68, 83-85, $87,88,90-92,93,249$, 274-278

functional/substantive factors, 4,5 , $11,12,25,27,68,88,92$, 93, 202, 237, 274

functional/substantive grounding, 4 , 5, 8-12, 23, 25-29, 34-38, 42, 43-48, 64, 66, 81, 85, 91, $93,116,148,149,264$, 273-278

initial syllable-see under strong positions

lexical access-see under speech processing licensing by cue, $83,86,88-92,257$, 276

long vowel-see under strong positions markedness

markedness constraints, defined, 3,32

positional markedness, 7,15 , 251, 252, 256-259, 260, 264, 265, 268-270, 277

noun-see under strong positions perceptual prominence, $3,4,8-13,23$, $24,25-27,34,42-50,55$, 60-62, 67, 69-71, 73, 81, 85, 
93, 95-97, 105, 109, 116, $126,135-137,140,143,147$, $148,196,233,237,252$, 259, 261-265, 268-272, 273, 277

phonological contrast, 8, 16-18, 34, $59,85,102,233,251,253$, 270,271

place features, 59, 87, 95, 140-143, 248,256

positional augmentation defined, 3-8 and perceptual salience, 3,4 ,

8-10; Ch 2 passim, especially $25,26,34,42-73$, 93; 95-97, 137, 143, 148, 252, 259, 261-265, 272, 273

See also constraints, augmentation positional constraints-see under constraints

positional faithfulness-see under faithfulness

positional markedness-see under markedness

positional neutralization, $8,13-15,18$, $22-24,31,86,88,92,96$, $109,147,149,150,188$, 196, 233, 245, 247-249, 251-270, 272, 274, 276

"reverse" positional neutralization, 8

Prominence Condition-see under Schema/Filter model prominent positions-see strong positions ranking permutations-see typology: factorial typology relativized constraints-see constraints: positional (relativized) constraints released consonant-see strong positions: syllable onset root-see under strong positions Schema/Filter model, 4, 5, 9, 11, 12, $24,25-38,39,42,45,48,81$, $85,87,88-92,93,116,143$, $247,275,278$

constraint filters, 4, 8-12, 23, 25-38, 41-48, 45, 48, 51, 73, $74,78,79,81-93,96,116$, $137,140,143,145,148$, 197, 202, 218-220, 230, 231-238, 252, 257, 263, 264, 273-278

constraint schemas, 4, 12, 28-35, $37-42,45,50,51,73,74,78$, $79,82,93,98,141,196$, 247, 261-263

Prominence Condition, 4, 5, 9, $10,12,23,24,26-29,31,36$, $37,42-81,82,84,88,91,93$, $95,96,116,126,135,137$, $140,143-146,148,198,238$, 249, 252, 259, 263, 273, 277 Segmental Contrast Condition, 4, $5,9-12,24,26,28,32,36$, $37,74,78,79,81-93,96$, 146, 147-149, 197-199, 202, 203, 218-220, 223, 227, 230-238, 247, 249, 252, 259, 263, 264, 273, 277

Segmental Contrast Condition-see under Schema/Filter model sonority, 4, 6, 9-11, 44, 53-70, 79, 80, 95-97, 102-104, 109-117, 126-140, 145, 156-174, 181-188, 198, 237, 239, 243, 
$246,249,256,262,263$,

$265,271,273,276,277$

high-sonority peaks, 109-116,

137-140

low-sonority onsets, 6, 9, 10, 60,

$62,67,95,96,103,116$,

127-135, 156-173, 181, 187,

$188,237,239,256,265,276$

sonority scale, 55-59, 64-67, 70,

$79,80,104,111,131,134$,

$137,145,157,174$

speech perception-see under speech

processing

speech processing

lexical access, 86, 204, 208, 216,

221,223

speech perception, $60,62,69,82$,

148-150, 203, 205, 209, 218,

$219,228,229,265$

word recognition, 4, 9-11, 24, 25,

26, 32, 81-88, 93, 147-150,

197-199, 201-207, 209-213,

215, 217-220, 222-238, 241,

$242,249,273,274$

stress, 6, 7, 9-11, 13-15, 49-54, 58,

$59,70-73,77,80,84,85,87$,

90, 96-109, 111-114,

116-118, 122-137, 148, 149,

$154,179,180,188-201,203$,

206, 207, 213, 216, 218-227,

229, 232, 249, 273, 274, 277

stressed syllable-see under strong

positions

strong positions

defined, 13-15

final syllable, 14, 86, 90, 107,

$108,124,125,136,141$, 222, 243, 244, 274

foot head, 15, 73, 75, 220, 221, 224, 245, 274 initial syllable, $4,7,10,11,15$,

$18-20,22,32,43,59,65,66$,

$74,76,80,82,84-86,89-92$,

$93,122,125,147-173$,

187-189, 198-203, 206-210,

218-220, 225, 226, 231-233,

238-249, 255, 256, 262, 265,

$271,273,274,277$

long vowel, $6,14,15,33,41,46$,

$55,74,75,81,87,95,100$,

$101,110,130,137-140,141$,

143-145, 147, 248, 273, 274,

276, 277

noun, 14, 192, 196, 197, 274

phonetically strong positions, 4 ,

$5,10,11,14,15,24,26,32$,

$36,75,76,78,81-84,86-93$;

Ch 3 passim, especially 95 ,

116, 137, 140, 143-146; 147, $148,197,241,242,249$,

274, 276, 277

psycholinguistically strong

positions, 4, 9-11, 14, 15,

$24,26,29,32,34,36,74-78$,

81-90, 92, 93, 95, 96, 146;

Ch 4 passim, especially

147-149, 188, 197-201,

202-204, 206, 210, 211 ,

218-220, 226, 227, 230-238,

241, 242, 247-249; 263, 273,

274, 277

root, $3,7,10,11,14,15,22,30$,

$34,38,39,71,72,74-78,82$,

$85,86,92,105,106,136$,

138, 139, 147-150, 188-199,

201-203, 206, 211-219, 227 ,

231, 233, 238-241, 248, 249,

255, 265, 273, 274, 277

stressed syllable, 3, 4, 6-8, 10 ,

$13-15,18,22,32,40,43,47$, 
49-54, 55, 59, 65, 67-71, 74, $75,79,81,82,86-91$, 95-137, 143-146, 147, 149, 187, 203, 206, 213, 216, 218-226, 230, 231, 241, 242, 251, 254-256, 261, 271, 273, 276, 277

syllable onset (released consonant), 14, 15, 43, 68, $69,74,75,78,80,81,87$, $89,95,140-145,147,271$, 274,276

substantive factors-see functional/ substantive factors substantive grounding-see functional/ substantive grounding syllable onset-see under strong positions

syllable structure, $5,67,102,150$, $154,160,173-187$

tone, $6,9,14,15,32,50,54,60$,

69-72, 82, 85, 90, 95-97, 103-109, 116, 129, 135, 149, 202, 218, 227-230, 232, 274, 276

typology

factorial typology, 15, 20, 21, $100,252,255,258,265-269$, 272

typological predictions, 3, 5, 21, $72,95,96,149,259,264$, 265

weak positions, $8,13-23,88,122$,

$251,252,255-260,264-272$ affix, 14, 22, 189-195, 211-218, 255,260

non-initial syllable, 19, 22, 156, $159,207,255,260$ unstressed syllable, $8,22,88$, $109,110,114,117,121$, 122, 207, 220, 222, 225, 251, 254-256, 268

word recognition-see under speech processing 